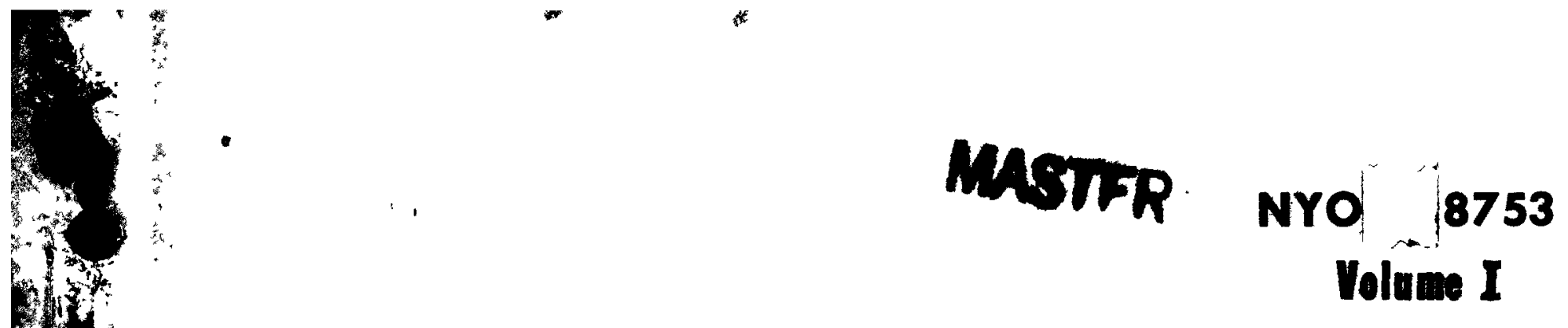

\title{
DESICN AND FEASIBILITY STUDY OF A
}

PEBble Bed ReACTOR-SteAM POWER PLANT

May 1, 1958

SANDERSON \& PORTER

Now York, N.Y. 


\section{LEGAL NOTICE}

This report was prepared as an account of government sponsored work. Neither the United States, nor the Commission, nor any person acting on behalf of the Commission:

A. Makes any warranty or representation, express or implied, with respect to the accuracy, completeness or usefulness of the information contained in this report, or that the use of any information, apparatus, method, or process disclosed in this report may not infringe privately owned rights; or

B. Assumes any liabilities with respect to the use of, or for damages resulting from the use of any information, apparatus, method, or process disclosed in this report.

As used in the above, "person acting on behalf of the Commission" includes any employee or contractor of the Commission to the extent that such employee or contractor prepares, handles or distributes, or provides access to, any information pursuant to his employment or contract with the Commission.

Price \$5.00. Available from the Office of Technical Services Department of Commerce, Washington 25, D.C. 


\section{DISCLAIMER}

This report was prepared as an account of work sponsored by an agency of the United States Government. Neither the United States Government nor any agency Thereof, nor any of their employees, makes any warranty, express or implied, or assumes any legal liability or responsibility for the accuracy, completeness, or usefulness of any information, apparatus, product, or process disclosed, or represents that its use would not infringe privately owned rights. Reference herein to any specific commercial product, process, or service by trade name, trademark, manufacturer, or otherwise does not necessarily constitute or imply its endorsement, recommendation, or favoring by the United States Government or any agency thereof. The views and opinions of authors expressed herein do not necessarily state or reflect those of the United States Government or any agency thereof. 


\section{DISCLAIMER}

Portions of this document may be illegible in electronic image products. Images are produced from the best available original document. 
NYO 8753

AEC Research and Development Report UC-81, Reactors-Power (TID-4500, 14th Ed.)

DESIGN AND FEASIBILITY STUDY

OF A

PEBBLE BED REACTOR-STEAM POWER PLANT

May 1, 1958

Work Performed under Contract No. AT(30-1)-2095

for the U.S. Atomic Energy Commission

by

SANDERSON \& PORTER

NEW YORK, N. Y. 
This report was prepared by the staffs of

Sanderson \& Porter Engineers

and

Alco Products, Inc.

Originally published as S \& P 1963A, Parts I and II 


\title{
CONTENTS
}

\author{
PART I PLANT DESIGN \\ Section 1.0 Design Concept and Summary \\ Section 2.0 Plant Design \\ Section 3.0 Reactor Fuel \\ Section 4.0 System Design \\ Section 5.0 Cost Analysis \\ Section 6.0 Drawings
}

PART II SYSTEM ANALYSIS

Section 1.0 Reactor Physics

Section 2.0 Thermal and Hydraulic Analysis

Section 3.0 Fuel Cycle Economics

Section 4.0 Steam Cycle Analysis

Section 5.0 Capital Cost Summary 



\section{Pebble Bed Reactor - Steam Power Plant \\ of 125 eMW}

\subsection{Design Concept \& Summary}

This report covers a design and feasibility study of a pebble bed reactor-steam power plant of 125 megawatt electrical output.

The design of this reactor system and its associated steam turbine generator plant is based on the premise that in order to produce economical and competitive nuclear power for central station use, a reactor system should have the following characteristics:

a) not temperature limited by the fuel elements, coolant or structural materials;

b) capable of raising steam suitable for use with modern steam turbine generating equipment;

c) fuel in a form which is easily fabricated, reprocessed and handled.

The reactor design which evolved from this study is a two-region thermal breeder, operating on the uranium-thorium cycle, in which all core structural materials are graphite. The reactor working fluid, or coolant, is helium. This combination of graphite, a high temperature refractory, and helium, an inert gas, effectively removes all practical temperature limitations from the system. Not being temperaturelimited, it is possible to generate steam suitable for use with the most modern steam turbine equipment. A cutaway perspective of the reactor is shown in Figure $1.0-1$.

Fuel is in the form of unclad spherical elements of graphite, containing fissile and fertile material. Blanket elements are graphite spheres containing fertile material only. Incorporation of the fissile and fertile material in refractory spheres offers a comparatively simple method of fuel fabrication which can be effected by mass production methods. Such a fuel form is amenable to simple handling techniques in loading and reprocessing. Randomly packed spheres in a reactor are a material form that is intermediate between the carefully fabricated, 
FIGURE $1.0-1$

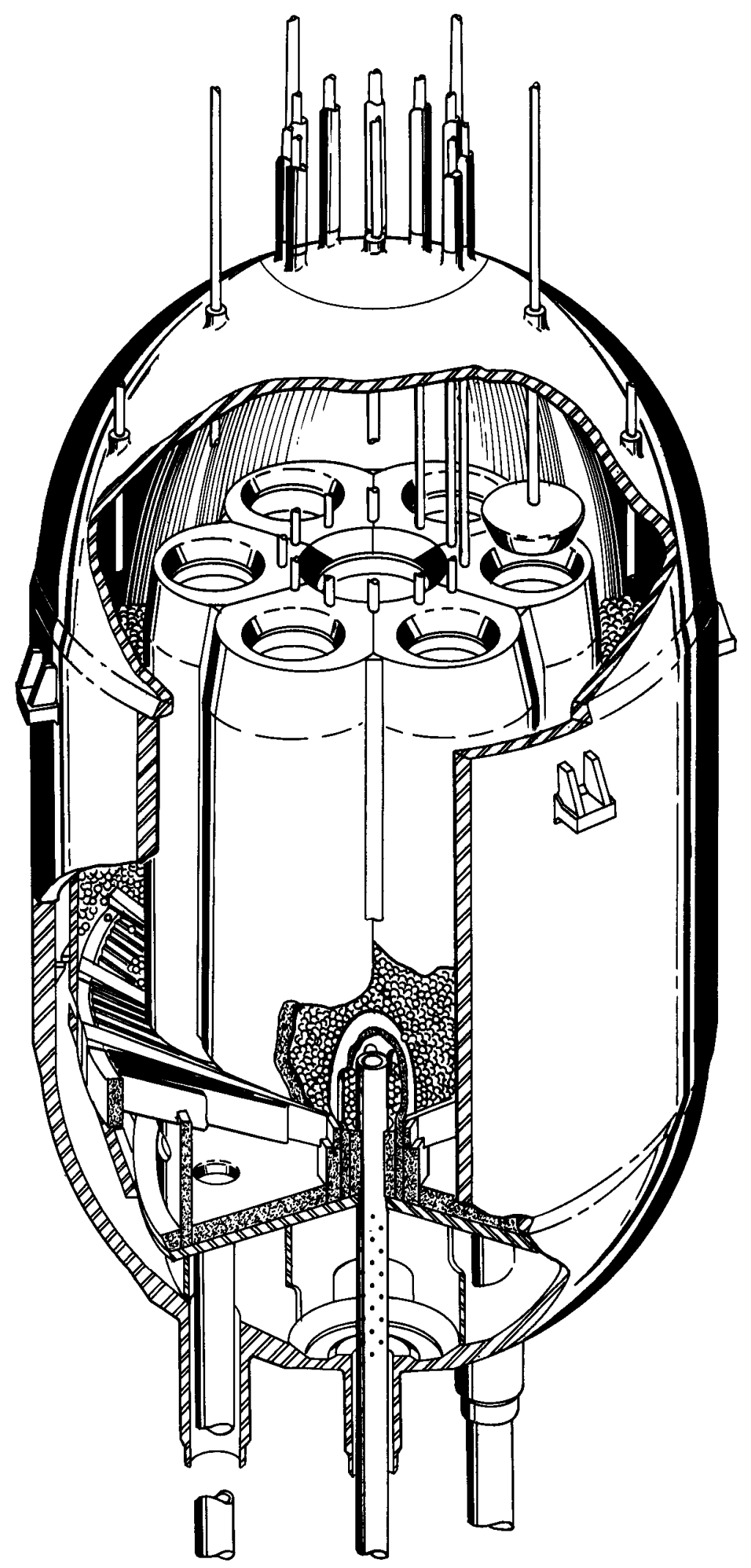

Cutaway PERSPECTIVE OF the REACtOR 
precisely located and firmly supported plates, rods and tubes used in most reactors and the completely unfinished and dispersed bulk material used in a homogeneous slurry reactor.

The power plant consists of a primary and secondary loop. Helium transports heat of fission from the reactor to a steam generator in which steam is raised. This steam is used in a secondary loop to generate electricity in conventional turbine generating equipment.

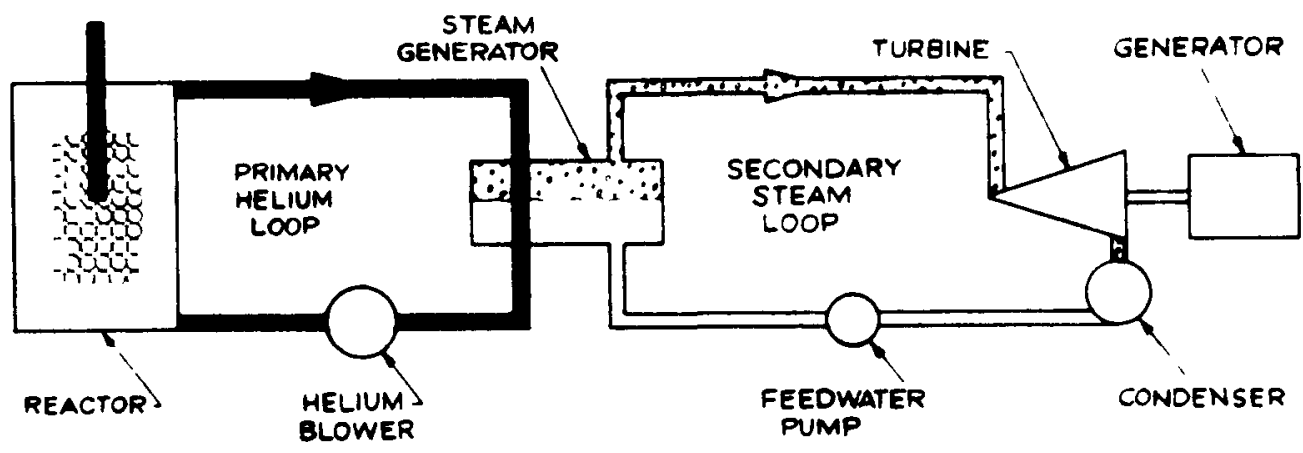

The primary loop consists of the reactor plus three steam generators and blowers operating in parallel. This equipment together with gas handling equipment which operates with radioactive gas is housed in a containment vessel as shown in the cutaway perspective of the primary system shown in Figure 1.0-2.

This study included a survey of the nuclear characteristics of cores suitable for reactors capable of outputs from 200 to 4000 megawatts of heat. These reactors are breeders in the larger sizes. The reactor selected for this design can have a conversion ratio of . 863 to $1.15 \mathrm{de-}$ pending upon the loading schedule and the validity of the U-233 crosssection data.

The design of the non-nuclear portion of the 125 eMW-PBR is based on conventional power plant practices. The plant is intended for base load operation, interconnected on a grid with other plants. The reactor is designed to produce $350 \mathrm{MW}$ of heat. At full load, $337 \mathrm{MW}$ is required to raise $1,055,000$ pounds per hour of steam to produce a net generating 
Figure 1.0-2

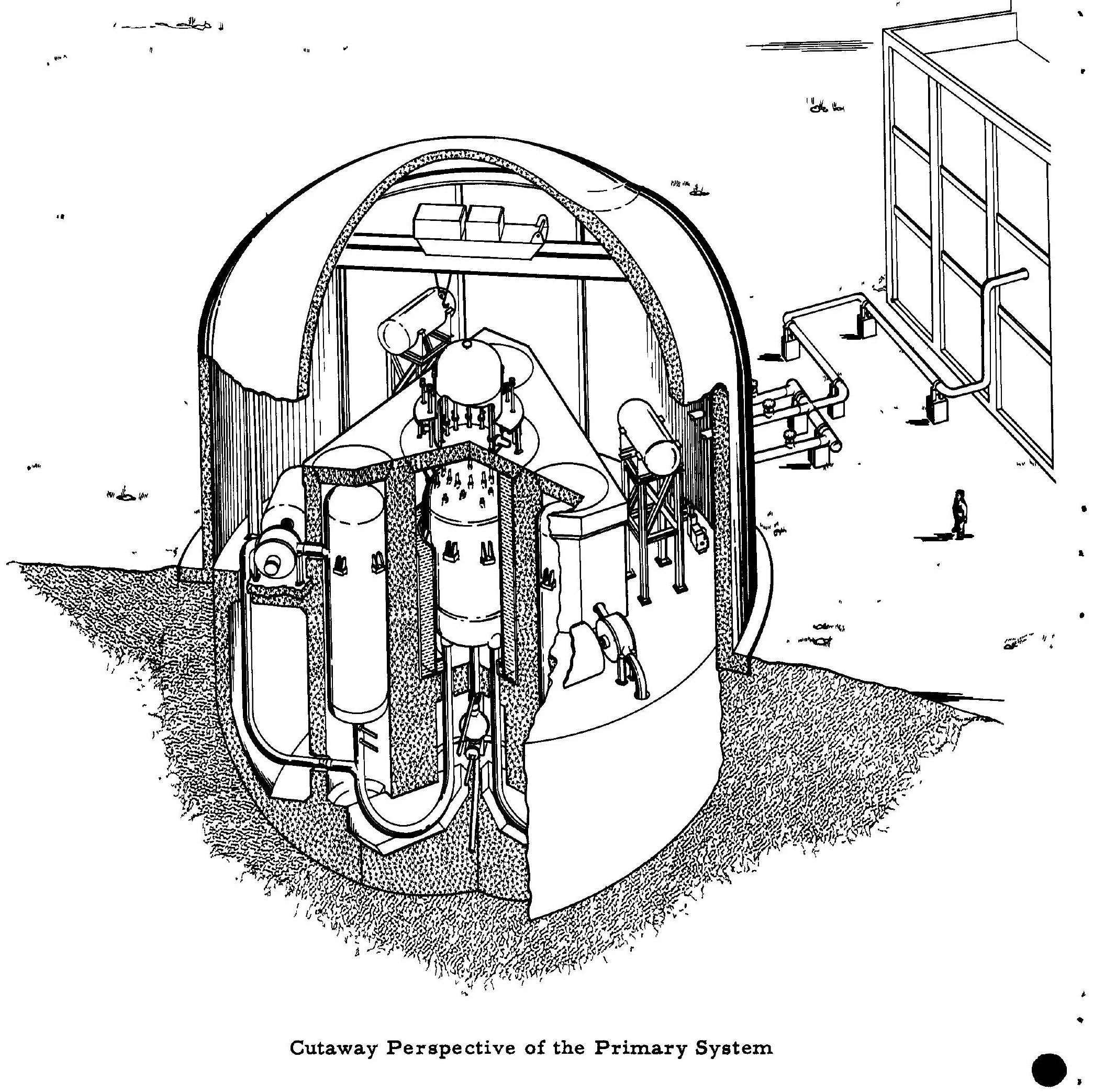


capability of $126,753 \mathrm{KW}$ in a $1450 \mathrm{psi} / 1000^{\circ} \mathrm{F}$ turbine generating plant at a net heat rate of $9077 \mathrm{BTU} / \mathrm{KWhr}$,

The capital costs of the 125 eMW-PBR are estimated at $\$ 32,221,000$, or at $\$ 254.20$ per kilowatt. Production costs, summar ized in Table 1.0-1, are between the limits of 7.46 and 8.07 mills per kilowatt hour, based on a conversion ratio of 0.863 .

This study has shown that a reactor system can be designed to operate with modern steam turbine generating equipment which, with the fuel concept used, makes it possible to generate competitive nuclear power. 
TABLE $1.0-1$

\section{5 eMW-PBR Data and Costs}

\section{General Data}

Net capability

Reactor heat output

Net station heat rate

Steam pressure

Steam temperature

Helium pressure

Investment Data ( $\$ / \mathrm{KW})$

Property \& improvements

Turbine plant structures

Turbine plant

Reactor plant structures

Reactor plant

Electrical \& miscellaneous

Total

Fuel Data $\left(k / 10^{6} \mathrm{BTU}\right)$

Fabrication

Inventory \& Burnup

Shipping

Reprocessing
$126,753 \mathrm{KW}$

$337,150 \mathrm{KW}$

$9077 \mathrm{BTU} / \mathrm{KWhr}$

1450 psi

$1000^{\circ} \mathrm{F}$

$950 \mathrm{psi}$

7.05

11.35

97.80

24.40

94.30

19.30

254.20

$\operatorname{Max}$

10.59

7.12

1. 50

$\frac{2.72}{21.93}$

Cost of energy (mills/KWhr)

Plant at $14 \%$ fixed charges

\& $80 \%$ load factor

$\frac{\text { Min. }}{4.31}$

7.12

1.00

Total $\frac{2.72}{15.15}$

Operation

Fuel
5.08

1.00

Total $\frac{1.38}{7.46}$
5.08

1. 00

$\frac{1.99}{8.07}$ 
Section 2.0

Plant Design

Page

2.1 Initial Design Basis.................... 2-1

2.2 Nuclear Survey ......................... 2-3

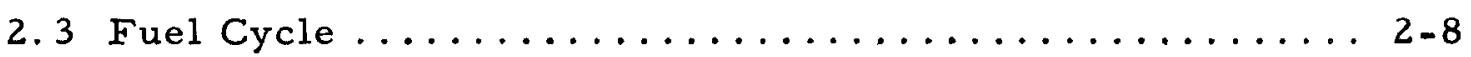

2.4 Fluid Dynamic and Thermal Survey .............. 2-10

2.5 Plant Heat Balance ...................... 2-12

2.6 Summary of Reactor Characteristics ............. 2-15 


\subsection{Plant Design}

\section{1 Initial Design Basis}

The contract under which this work was performed specified that "the contractor should investigate and determine the feasibility of a $125 \mathrm{MW}$ steam electric plant using a high temperature gas-cooled pebble bed reactor as a heat source."

Based on previous work done by the contractor and associates the basic design of the reactor had been established as being of graphite construction and using helium as the heat transport fluid. In order to size the reactor for the nuclear analysis it was necessary to select a set of steam conditions for the turbine generator system. The steam cycle analysis is reported in Section 4, Part II of this report and as a result of this study a $1450 \mathrm{Lb} / 1000^{\circ} \mathrm{F}$ steam cycle with $400^{\circ} \mathrm{F}$ feed water temperature was selected. Such a plant would have a heat rate of the order of $9100 \mathrm{BTU} / \mathrm{KWhr}$, resulting in a gross heat input, including energy added to circulate the helium, of $335 \mathrm{MW}$. Knowing the plant would have a capability in excess of $125 \mathrm{eMW}, 350 \mathrm{MW}$ of reactor heat was selected for design. A $200^{\circ} \mathrm{F}$ approach to the $1000^{\circ} \mathrm{F}$ steam and a $50^{\circ}$ approach to the evaporator section in the steam generator resulted in a reactor outlet temperature of $1200^{\circ} \mathrm{F}$ and an inlet temperature of $500^{\circ} \mathrm{F}$ for a temperature rise of $700^{\circ} \mathrm{F}$ and a flow of $1,360,000$ pounds of helium per hour. The $500^{\circ} \mathrm{F}$ reactor inlet temperature was found to produce lower net power costs than a system with fewer feedwater heaters (i.e. lower feedwater temperature) and a lower reactor inlet temperature. Furthermore, since none of the graphite parts will be lower than the helium inlet temperature $\left(500^{\circ} \mathrm{F}\right)$, radiation damage to the se parts should be minimized. In the initial survey heat added by the gas circulating blower was neglected as was any temperature rise in the reactor inlet helium due to heat transfer through piping.

Initial design conditions are summarized in Table $2.1-1$. 
TABLE 2. 1-1.

\section{Initial Design Conditions}

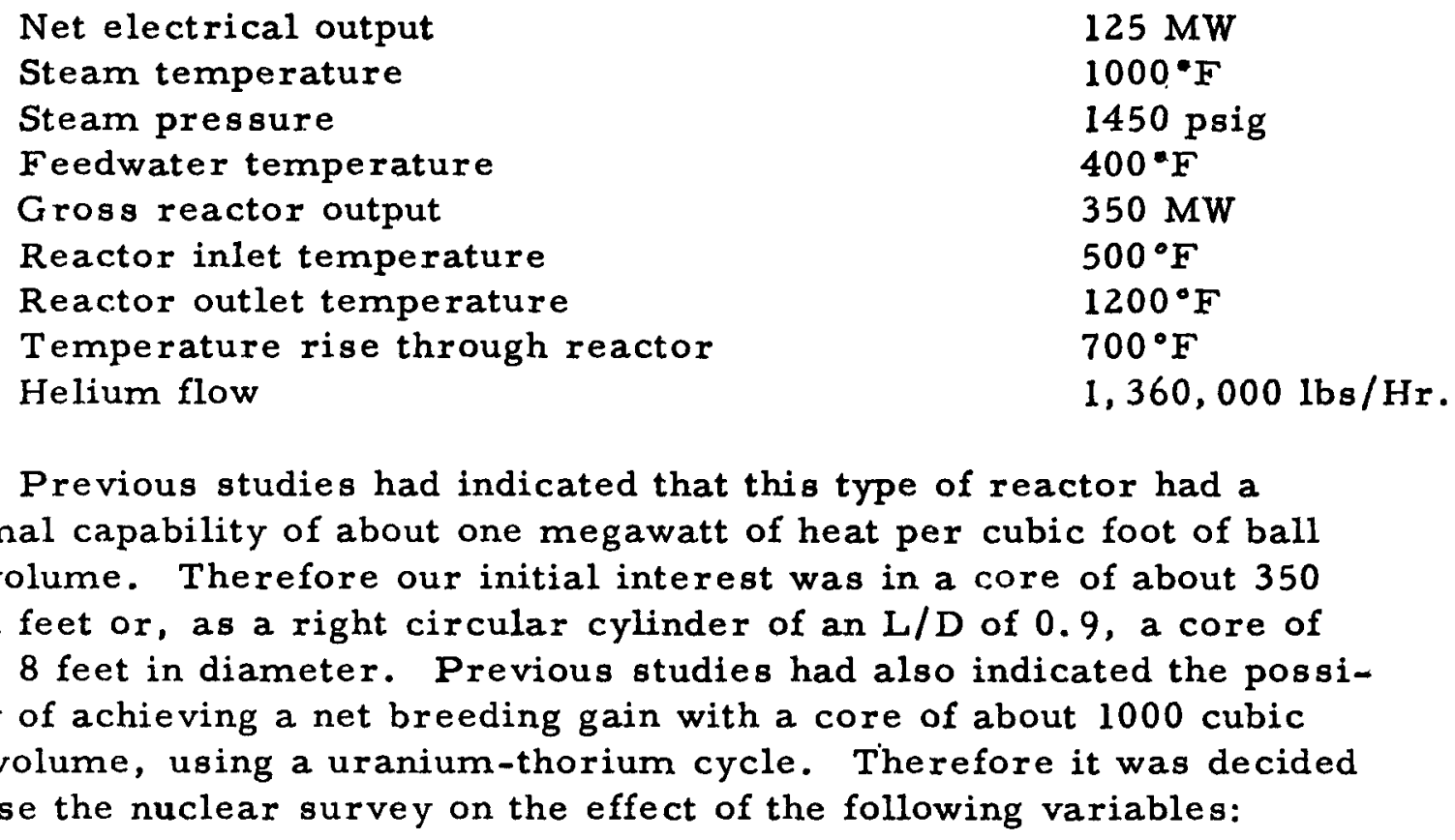

TABLE 2.1-2

Variable

Core diameter, feet

Weight percent of oxide in fuel elements Percent voidage in core $\mathrm{Th} / \mathrm{U}$ atom ratio
Range

$7-20$

$7.5-30$

$10-39$

$5-50$

The Thorium-Uranium-233 fuel cycle was selected as a basis for the $125 \mathrm{eMW}-\mathrm{PBR}$ because it was expected that the higher value of $\eta$ for U-233 (i.e. neutrons produced/nentrons captured in fuel) would make this cycle more favorable than the Uranium-Plutonium cycle. During the course of this study, it became apparent that a wide discrepancy exists in the values of $\eta$ for U-233 as a function of energy. This discrepancy is best illustrated in Figure $1.2-6$ on page 1-45 of Part II. This figure shows values of a for U-233 (non-fission captures/ 
fission captures) as a function of energy as calculated by R. L. Murray for Alco Products using U-233 cross-section data from BNL-325, Suppl. 1. Also plotted on this figure are the somewhat lower values of a based on ORNL data (Ref.22, Section 1.0, Part II). The value of a, averaged over the energy spectrum of the 125 eMW-PBR is 0.257 based on the Murray data. This results in a value of $\eta=2.02$. Using the ORNL data, $a$ is 1433 giving a value of $\eta$ of 2.22 . Since $\eta$ must be at least 2.0 in order to achieve breeding even in an ideal system having no extraneous neutron losses, it can be seen that the design could range between one with essentially no possibility of breeding to one that should obtain a net breeding gain depending on the true values of the U-233 cross-section. In order to present a conservative picture of the possibilities of this system, the Murray data has been used in the final analysis.

\subsection{Nuclear Survey}

The principal items of interest resulting from the nuclear parameter survey are the initial breeding ratio and the effective multiplication factor. For those fuel compositions which resulted in a low conversion ratio or $\mathrm{K}_{\mathrm{eff}}<1$, the only interest was in the trend indicated. The results have been expressed graphically in a series of plots and cross plots, details of which are to be found in Section 1, Part II.

Figures 2.2-1 through 2.2-4 are typical of the results obtained in this parametric survey. Figures 2.2-1 and 2.2-2 show the effect of $\mathrm{Th} / \mathrm{U}$ ratio and voidage with two different oxide fractions for a 9 foot diameter core. Figure 2.2-3 shows the effect of core diameter on critical mass. Figure 2.2-4 shows the effect of core diameter on the initial breeding ratio.

The selection of the final core was based on such factors as core loading (fuel inventory cost), weight percent of $\mathrm{ThO}_{2}+\mathrm{UO}_{2}$ in core balls as it affects methods of fabrication, pumping power, IBR and $K_{e f f}$.

Fuel inventory and capital cost place an upper limit on core size, while pumping power places a lower limit. It was felt that 10 weight percent or less of oxides in the core balls was a desirable objective because this would remove any restriction on method of fabrication as discussed in Section 3.1.1. An initial $\mathrm{K}_{\text {eff }}$ of about 1.10 was felt desirable in order that a reasonable core lifetime might be attained. Obviously, a high IBR was also desirable. 


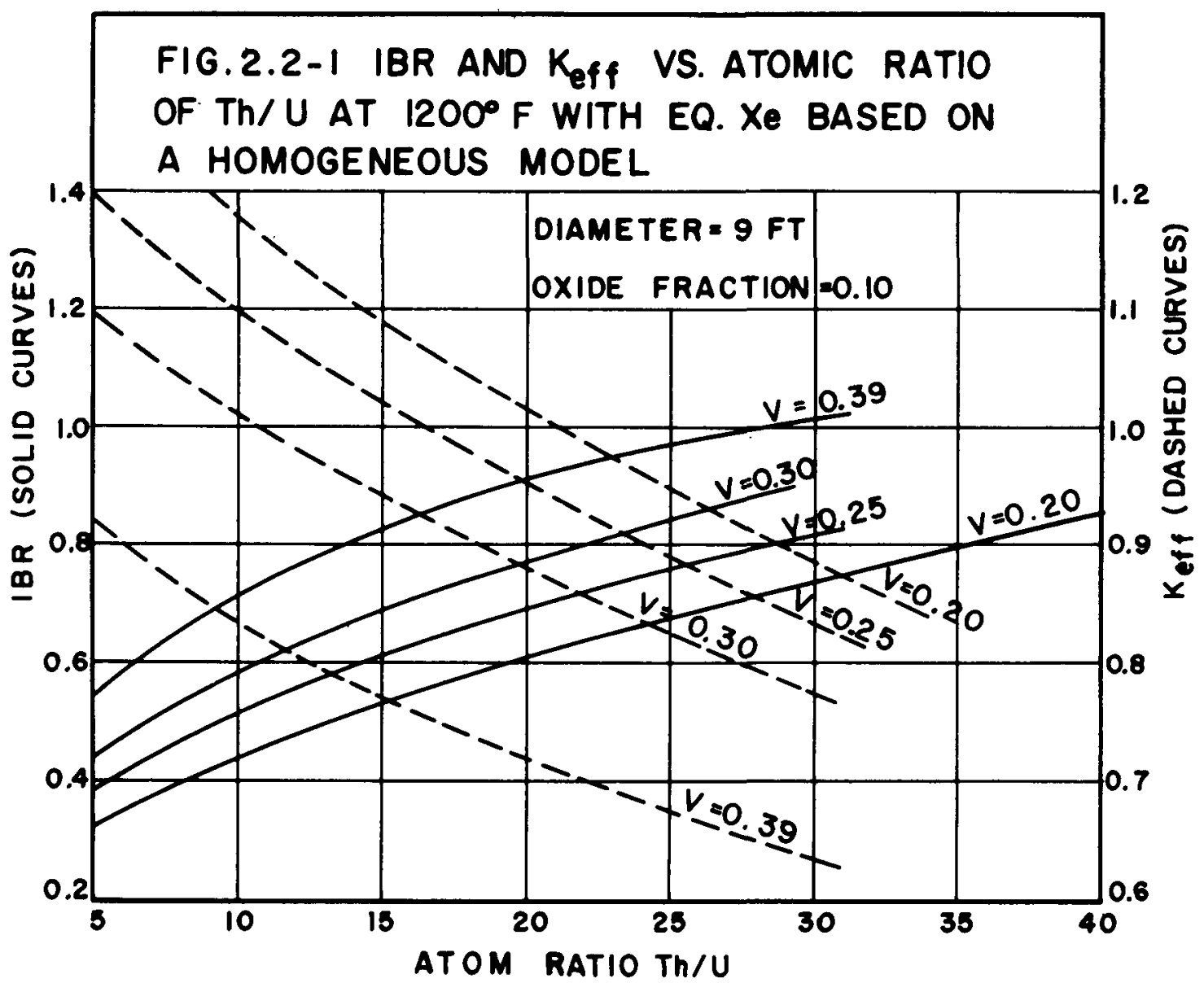

FIG.2.2-2 IBR AND $K_{\text {off }}$ VS. ATOMIC RATIO OF Th/U AT $1200^{\circ} F$ WITH EQ.Xe BASED ON A HOMOGENEOUS MODEL

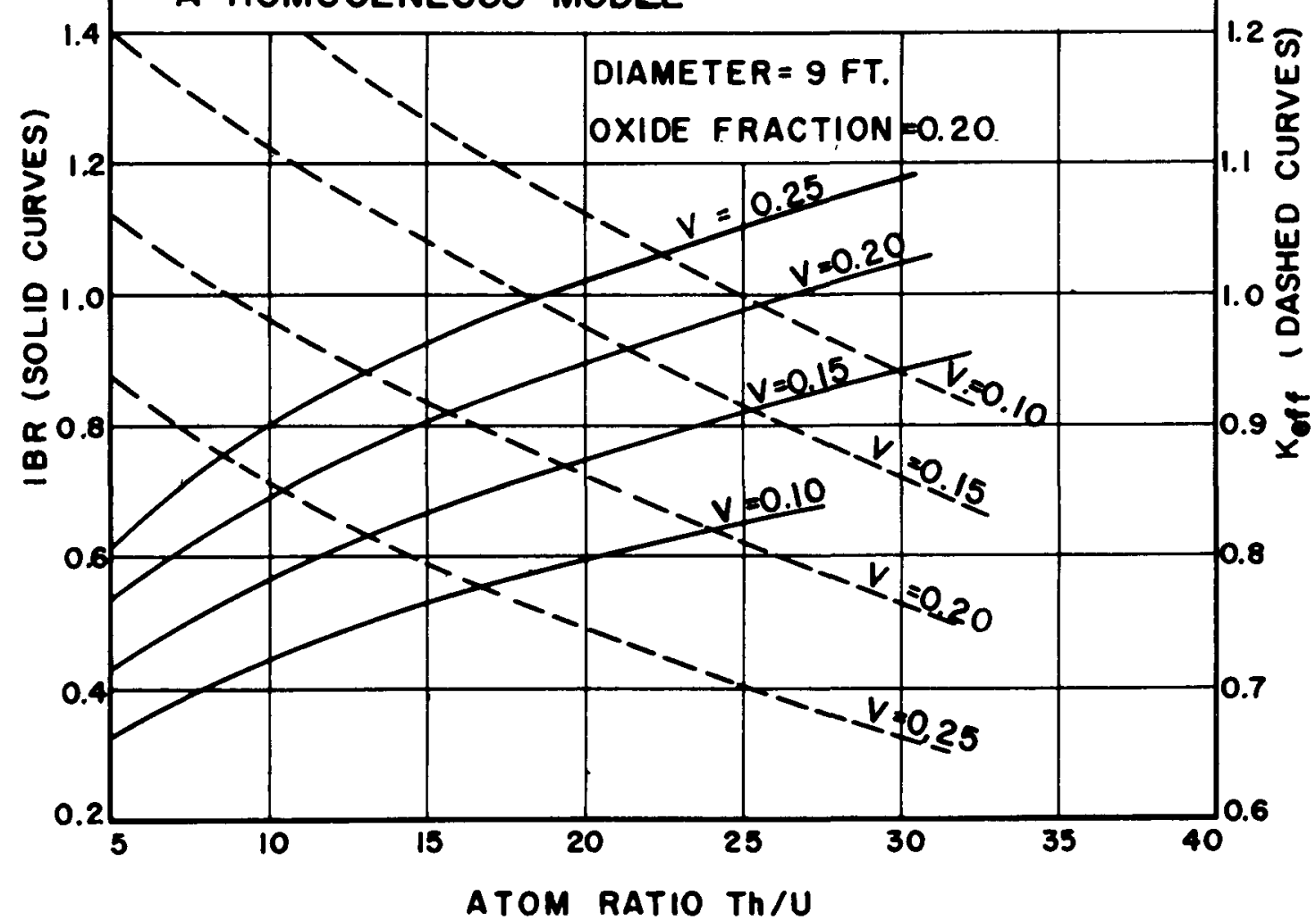



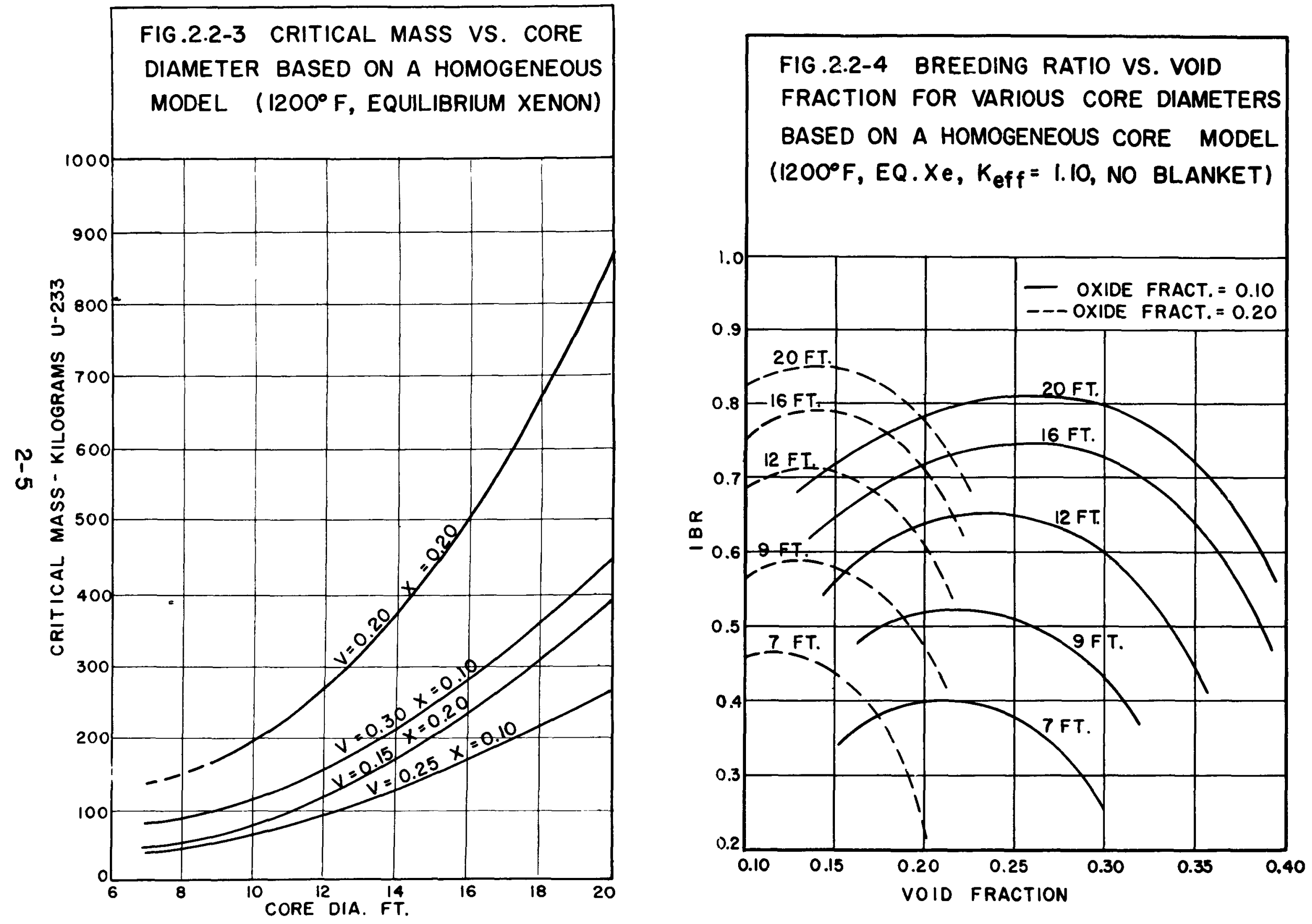
Fuel cycle costs and pumping power limitations bracketed cores of interest between 8 and 10 feet in diameter. Selecting a pumping power of $1-1 / 4 \%$ of the reactor output resulted in a series of the following cores:

TABLE $2.2-1$

Core Diameter vs. Void Fraction - 1-1/4\% Pumping Power

Diameter Void

839.0

$8-1 / 2 \quad 34: .0$

929.0

$9-1 / 2 \quad 25.5$

$10 \quad 21.5$

Since the reactor core is already undermoderated and any increase in void fraction tends to make it more so, and since $\eta$ and the IBR are improved by making the reactor more thermal, the smaller cores, i.e. 8 feet, were rejected. On the other hand, increasing the diameter causes the blanket volume (for constant thickness) to increase rapidly with an adverse affect on overall economics.

Balancing these several factors resulted in the selection of a 9 foot diameter core having a $29 \%$ net voidage. Detailed nuclear calculations were then made which included the effect of lumping some of the graphite. Figure 2.2-5 shows the resulting $I B R$ and $K_{\text {eff }}$ as a function of $T h / U$ atom ratio. A fuel composition having a $\mathrm{Th} / \mathrm{U}$ atom ratio of 11 was finally selected since the resulting $K_{\text {eff }}$ of 1.1 indicated a reasonable core lifetime. The results of subsequent core lifetime calculations showed that at the end of 100 full power days of operation, $K_{\text {eff }}$ would fall below 1.0 , thus terminating the core life. It should be noted that atom burnup (atoms fissioned/all atoms initially present in the fuel element) at this point, was only $0.021 \%$. Thus, it would be expected that reactivity loss rather than burnup would be the limiting factor in fuel element lifetime.

A detailed mechanical description of the reference design is given in Section 4.2. Principle nuclear characteristics are included in Table 2. 6-1. 


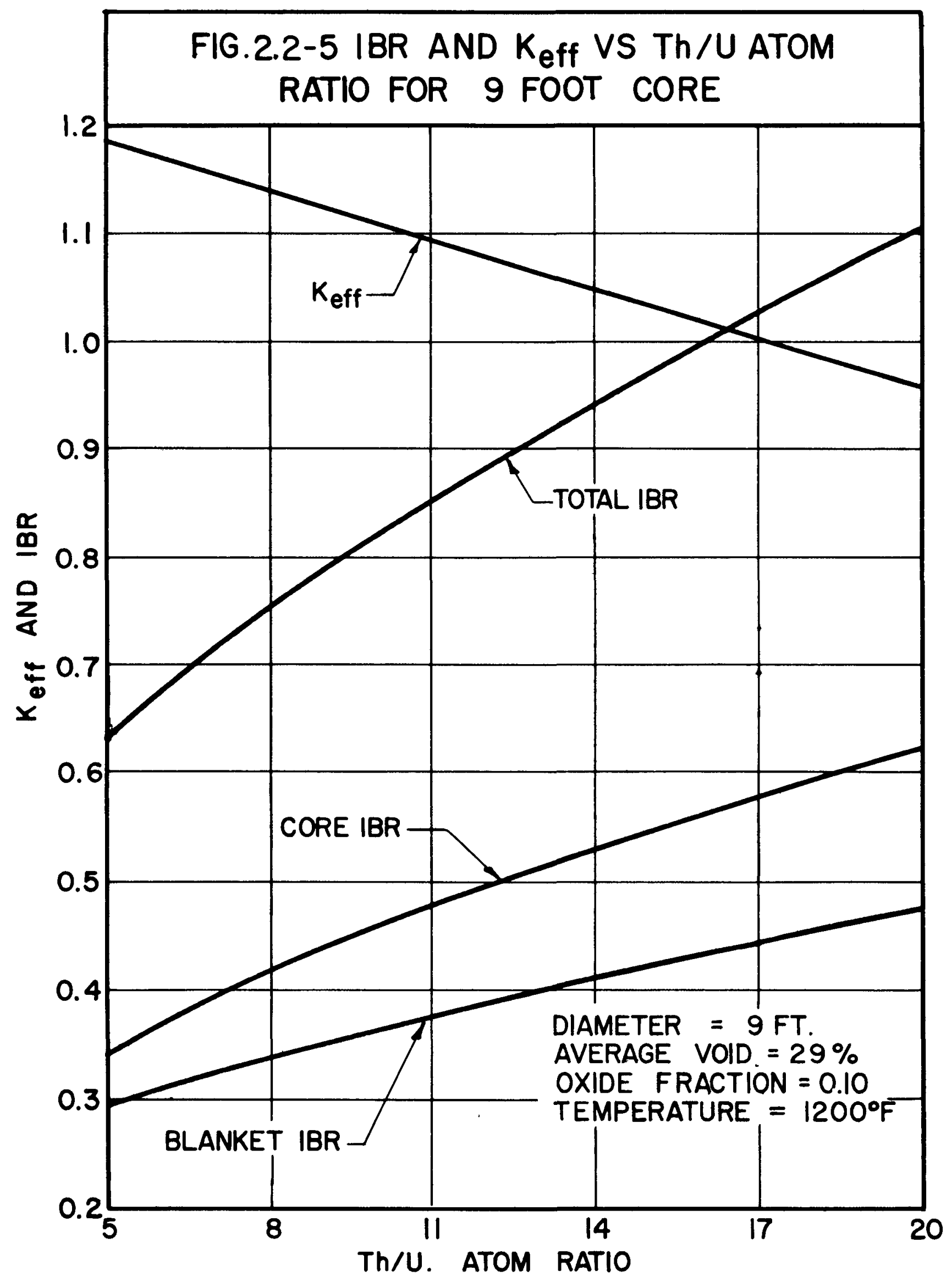

2-7 


\section{3 Fuel Cycle}

Figure 2.3-1 is a flow sheet for the fuel cycle based on the reference design. Since we have selected a two region reactor, there are two distinct cycles shown, one involving the core and one the blanket. The core loading consists of graphite spheres which contain the oxides of thorium and U-233 in the atom ratio of $11: 1$ while the blanket loading consists of graphite spheres containing only thorium oxide. The material balance is based on a blanket use cycle of 1000 full power days (i.e. 10 core lifetimes, where 1/10 of the blanket is processed with each core loading). This decision is based on an economic analysis given in Section 3.0 of Part II.

Since spherical fuel elements possess a high degree of mobility making it relatively easy to load and unload such fuel, it is possible to consider fuel cycles involving continuous refueling. Among the advantages of such a mode of operation is the higher conversion ratio obtainable resulting from the absence of control rods required for reactivity control. The details of continuous refueling are discussed in Section 3.2, Fuel Handling.

All of the nuclear and economic calculations in this report are based on the use of U-233 as fissile material. We recognize that at present it would be necessary for such a reactor to operate on an initial loading of U-235 and thorium and to continue adding U-235 until a sufficient inventory of $U-233$ is built up to permit operation on $U-233$ alone. Table 2.3-1 below compares the characteristics of the PBR as designed with the same core but loaded with U-235 instead of U-233.

TABLE 2.3-1

Characteristics of 125 eMW-PBR Fueled with U-235 or U-233

\begin{tabular}{lll} 
& \multicolumn{1}{c}{$\mathrm{U}-235$} & $\mathrm{U}-233$ \\
\cline { 2 - 3 } $\mathrm{K}_{\mathrm{eff}}\left(1200^{\circ} \mathrm{F}, \mathrm{Eq} . \mathrm{Xe}\right)$ & 1.10 & 1.10 \\
$\mathrm{Th} / \mathrm{U}$ & $7.1: 1$ & $11: 1$ \\
$\mathrm{Kg} \mathrm{U}$ & 135.5 & 90.2 \\
$\mathrm{IBR}$ (core) & .44 & .477 \\
$\mathrm{IBR}$ (blanket) & .353 & .353
\end{tabular}

Thus it is apparent that if $U-235$ must be used initially, the rateof buildup of U-233 will be below that which would prevail ultimately. Subsequent core loadings would contain a mixture of U-233 and U-235. 
Figure 2.3-1

\section{Diagrammatic Flow Sheet of Fuel Cycle}

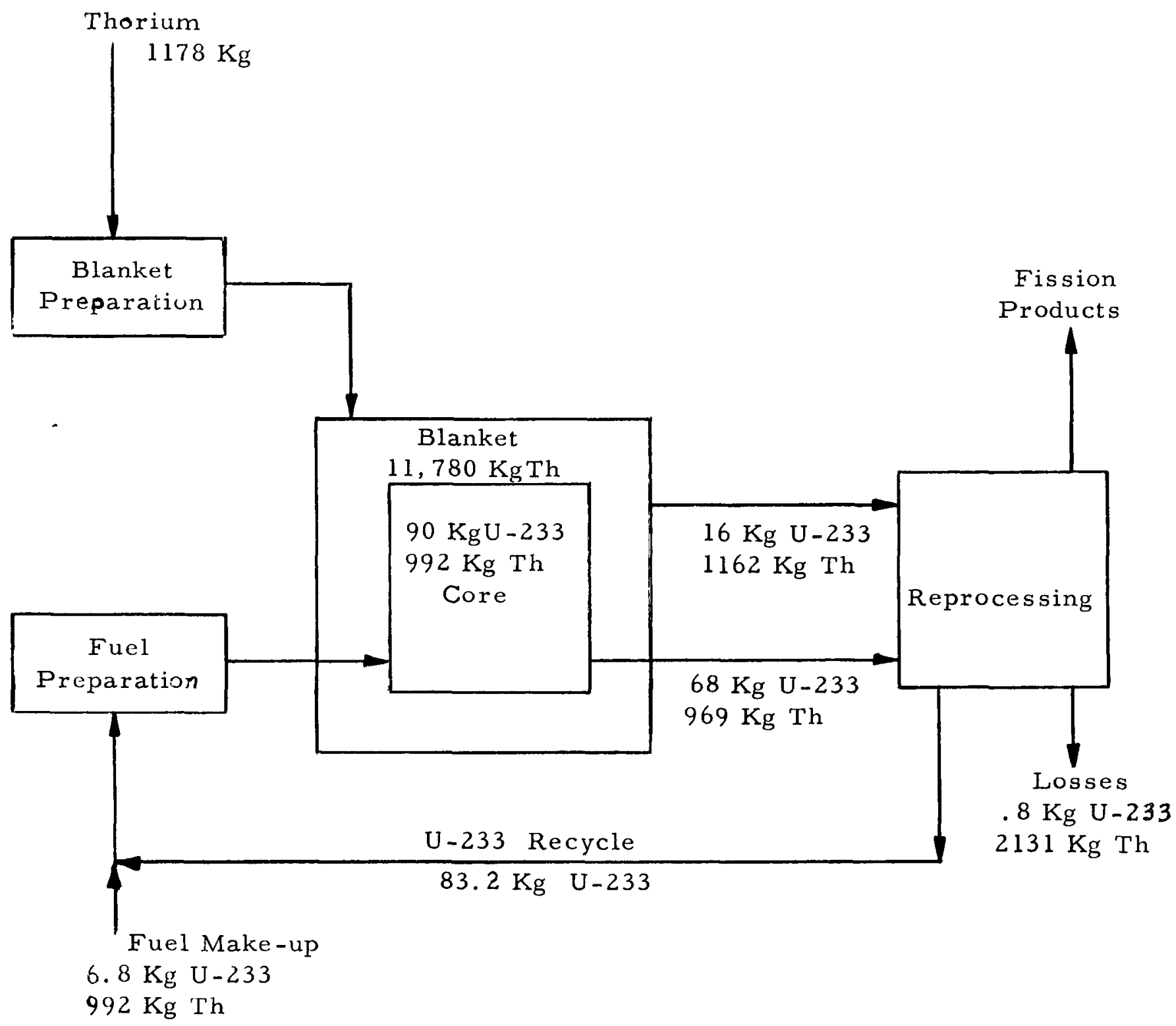

Equilibrium Conditions

100 day Core Life - 1000 day Blanket Life

Based on Disposal of all Thorium 


\subsection{Fluid Dynamic \& Thermal Survey}

Helium has been selected as the coolant for the PBR on the basis that it is the only gas having a good pumping power/heat transfer modulus that will not react with the PBR core materials. It is a completely inert coolant and thus releases the PBR from corrosion problems that face most other reactor concepts.

The voidage of a randomly packed bed of spherical particles is equal to $39 \%$. The nuclear survey of cores for the $350 \mathrm{tMW}-\mathrm{PBR}$ has shown that it is necessary to add more graphite to the core thus reducing this voidage which has been done by assuming it to be uniformly dis persed in the ball bed. From the standpoint of mechanical design this additional graphite has been used for structural purposes, in the form of graphite cylinders in which fuel balls are carried.

While the average voidage of a randomly packed bed is $39 \%$, the characteristic voidage is of the order of $38 \%$, the difference being due to wall effect. This difference in radial voidage results in a radial velocity profile through the bed where flow is greater at the wall than in the center of the bed. Placing the fuel in channels results in a flux peaking at the wall with minimum flux at the channel centerline. Thus the point of maximum flux coincides with the point of maximum flow and vice versa. This combined effect of flux and voidage distribution in a fuel channel is such as to flatten out the temperature profile in any given channel.

The 125 eMW PBR reactor consists of a central core surrounded by a blanket. Flow is through the blanket and core in parallel. The rate of heat generation in the blanket varies with time. Initially, about $5 \%$ of the reactor power is generated in the blanket due to gamma heating. After 1000 full power days, the blanket heat generation will be $13.5 \%$ of reactor power due to fissioning in the bred U-233. The most severe design condition occurs at startup, when the core is contributing $95 \%$ of the heat and the blanket $5 \%$. This condition has been used for design.

Allowing uniform flow through the core and blanket results in the lowest pumping power but in maximum fuel element temperature as well as extreme radial temperature variations of the helium leaving the core. Dividing the flow between the core and blanket in proportion to the heat generated results in uniform fuel element temperatures and temperature 
profile leaving the core but a high pumping power. Therefore the area between these two limits was explored and a final design evolved in which $67.5 \%$ of the flow passed through the core to remove $95 \%$ of the heat and $32.5 \%$ of the flow passed through the blanket to remove $5 \%$ of the heat.

This resulted in a $108^{\circ} \mathrm{F}$ temperature rise through the blanket and a temperature rise through the core varying from 410 to $1340^{\circ} \mathrm{F}$, depending upon the radial position. Maximum temperature drop through the film is $218^{\circ} \mathrm{F}$ and maximum fuel element surface temperature is $2170^{\circ} \mathrm{F}$. These temperatures exist only at startup and decrease with time, as the percentage of heat generated in the blanket increases.

The reactor pumping power is $2765 \mathrm{KW}$ for removal of $350 \mathrm{MW}$ of heat or a ratio of pumping power to heat transferred of $0.78 \%$. The total system pumping power is $8000 \mathrm{KW}$ and the installed blower power is $8526 \mathrm{KW}$. 


\section{5 Plant Heat Balance}

A heat balance for the total plant is presented in Drawing 1963-C-16. Principal data are summarized in the following table:

\section{TABLE 2. 5-1}

\section{Plant Heat Balance}

Reactor output

Power to blower (gas horsepower)

Heat to boiler

Gross capability

Gross heat rate

Auxiliary power

Net capability

Net heat rate
337. $15 \mathrm{MW}$

$7.67 \mathrm{MW}$

$344.82 \mathrm{MW}$

$139,029 \mathrm{KW}$

$8464 \mathrm{BTU} / \mathrm{KWhr}$

$12,276 \mathrm{KW}$

$126,753 \mathrm{KW}$

$9077 \mathrm{BTU} / \mathrm{KWhr}$

The primary system state points and heat balance are given in Table 2.5-2 while Table 2.5-3 presents the primary system volumes and transit time.

A flow diagram of the primary system is given in Drawing 1963-B-5 while a flow diagram of the secondary system is shown in Drawing 1963-C-17. 
TABLE 2.5-2

SYSTEM STATE POINTS \& HEAT BALANCE

378 Lbs/Sec Helium Flow

\begin{tabular}{|c|c|c|c|c|c|c|c|c|c|c|}
\hline Item & Location & $\begin{array}{c}\text { Temp } \\
{ }^{\circ} \mathrm{R}\end{array}$ & $\begin{array}{l}\text { Press. } \\
\text { PSIA }\end{array}$ & $\begin{array}{l}\text { Density } \\
\text { Lbs } / \mathrm{Ft}^{3}\end{array}$ & $\begin{array}{c}Q \\
\mathrm{Ft}^{3} / \mathrm{sec}\end{array}$ & $\underset{F t^{2}}{A}$ & $\begin{array}{c}\text { Velocity } \\
\text { Ft/sec }\end{array}$ & $\frac{\Delta P}{p s i}$ & $\stackrel{T}{\cdot F}$ & $\begin{array}{r}\text { Heat } \\
\text { MW }\end{array}$ \\
\hline 1 & Reactor Inlet & 1025 & 994.1 & .362 & 1045 & 4.065 & 257 & $-5.9($ duct $)$ & & \\
\hline 2 & Reactor Outlet & 1710 & 978.6 & .214 & 1765 & 3.99 & 442 & -15.5 & +685 & +342 \\
\hline 3 & Steam Generator Inlet & 1667 & 975.5 & .218 & 1735 & 3.99 & 435 & -3.1 (duct) & & \\
\hline 4 & Steam Generator Outlet & 966 & 969.1 & .374 & 1010 & 3.66 & 276 & $-6 \cdot 4$ & -701 & -350 \\
\hline 5 & Blower Inlet & 966 & 969 & .374 & 1010 & 3.66 & 276 & $-.1($ duct $)$ & & \\
\hline 6 & Blower Discharge & 982 & 1000 & .380 & 995 & 3.66 & 272 & -31.0 & +16 & \\
\hline
\end{tabular}


TABLE 2.5-3

SYSTEM VOLUME \& TRANSIT TIMES

\begin{tabular}{|c|c|c|c|c|c|c|c|}
\hline Item & Description & No. & $\frac{\text { Temp. }}{{ }^{\circ} \mathrm{R}}$ & $\frac{\text { Press. }}{\text { PSIA }}$ & $\frac{\mathrm{Vol}}{\mathrm{Ft}^{3}}$ & $\frac{\text { Weight }}{\text { Lbs. }}$ & $\begin{array}{l}\text { Transit } \\
\text { Time } \\
\text { millisec }\end{array}$ \\
\hline 1 & Blower Discharge Duct & 3 & 981 & 1000 & 9 & 3.4 & 9 \\
\hline 2 & Duct to Reactor & 3 & 992 & 997 & 3 & 124.9 & 330 \\
\hline 3 & Reactor Bottom Space & 1 & 1024 & 994 & 96 & 34.8 & 92 \\
\hline 4 & Reactor Slide Annulus & 1 & 1027 & 994 & 129 & 46.6 & 123 \\
\hline 5 & Reactor Top Plenum & 1 & 1031 & 994 & 640 & 230.4 & 457 \\
\hline 6 & Core & 1 & 1371 & 987 & 328 & 88.2 & 233 \\
\hline 7 & Grate \& Plenum & 1 & 1710 & 980 & 197 & 42.2 & 112 \\
\hline 8 & Duct to Generator & 3 & 1676 & 977 & 315 & 41.9 & 175 \\
\hline 9 & Steam Generator & 3 & 1311 & 973 & 2850 & 790.0 & 2090 \\
\hline 10 & Generator Side Annulus & 3 & 963 & 970 & 615 & 231.3 & 612 \\
\hline 11 & Inlet to Blower & 3 & 966 & 970 & 15 & 5.6 & 15 \\
\hline 12 & Blower & 3 & 973 & 985 & 102 & 38.5 & 102 \\
\hline & Total & & & & 5629 &, 677.8 & 4350 \\
\hline
\end{tabular}


2.6 Summary of Reactor Characteristics

TABLE 2.6-1

Reactor Characteristics of Final Design

Reactor Core

Equivalent Diameter

Height

Voidage

Moderator \& Structure

Number of fuel channels

Fixed moderator volume

Ball bed volume

Total volume

$9.0 \mathrm{Ft}$.

8. $1 \mathrm{Ft}$.

$29.0 \%$

Graphite

7

$130 \mathrm{Ft}^{3}$

$383 \mathrm{Ft}^{3}$

$513 \mathrm{Ft}^{3}$

Reactor Blanket

Equivalent thickness

Height

Voidage

Ball bed volume

1. $33 \mathrm{Ft}$.

$9.6 \mathrm{Ft}$.

$39.0 \%$

$496 \mathrm{Ft}^{3}$

Fuel Elements

Form

Spherical

Diameter, in.

$1-1 / 2$

Number

228,600

Composition, by weight

Graphite

$\mathrm{ThO}_{2}$

$\mathrm{UO}_{2}$

Total
Total, Kg.
11085.0
1129.0
1.02 .6
1231.6 .6

Unit, gr.

48.49

4.94

.45

53.88

Blanket Elements

Form

Diameter, in.

Spherical

Number

$3 / 4$

Composition, by weight

Graphite

Total, $\mathrm{Kg}$

13404.8

$2,370,000$

$\mathrm{ThO}_{2}$

13404.8

Unit, gr.

$\%$

5.66

50

26809.6

5.66

50

11. 32 
TABLE 2.6-1 (continued)

Nuclear

Core $\mathrm{C} / \mathrm{Th} / \mathrm{U}$ atom ratio

$3745 / 11 / 1$

Blanket $\mathrm{C} / \mathrm{Th}$ atom ratio

$22 / 1$

Critical Mass $\left(1200^{\circ} \mathrm{F}, \mathrm{Eq}\right.$. Xe), Kg. U-233

60.2

U-233 Loading, $\mathrm{Kg}$.

90.2

$\mathrm{Th}-232$ loading in core, $\mathrm{Kg}$.

992.2

Th-232 loading in blanket, $\mathrm{Kg}$.

11,780

Fuel burnup rate, $\mathrm{Kg} / \mathrm{MWYear}$

0.48

Initial capture to fission ratio

0.257

Fraction of epithermal fissions

0.372

Initial breeding ratio, core

0.477

blanket

0.353

Total

0.830

Average breeding ratio over core lifetime

0.863

Average power density, watts/cc

23.7

Atom burnup (\% total atoms)

Core (after 100 days)

0.021

Blanket (after 1500 days)

0.101

Temperature Coefficient

$-2.7 \times 10^{-5} /{ }^{\circ} \mathrm{F}$

Initial Multiplication Factor

$\mathrm{K}_{\mathrm{eff}}\left(1200^{\circ} \mathrm{F}, \mathrm{Eq} . \mathrm{Xe}\right) *$

1. 100

$\mathrm{K}_{\mathrm{eff}}\left(1200^{\circ} \mathrm{F}, \mathrm{Eq} . \mathrm{Xe}\right) * *$

1.070

$\mathrm{K}_{\text {eff }}\left(77^{\circ} \mathrm{F}\right.$, No Xe)

Average Thermal Flux at Startup, $\mathrm{n} / \mathrm{cm}^{2}-\mathrm{sec}$

Average Thermal Flux, after 100 days, "

1. 204

1. $3 \times 10^{14}$

2. $2 \times 10^{14}$

Control Rods

Nurnber

O.D. , in.

I. D. , in.

Material

Length, in.

Weight, lbs.

Drive

Reactivity Worth, initial

12

4

$3-1 / 2$

Haynes -25

99

83

Electric Motor

$17.3 \%$

* Equivalent bare homogenized core model

** Heterogeneous multiregion with equilibrium blanket composition. 
TABLE 2.6-1 (continued)

Thermal and Fluid Dynamic Characteristics

Design Power Level, MW 350

Design Pressure Level, psia 1000

Design Helium Flow, lbs/hr

Volumetric Heat Generation, $\mathrm{MW} / \mathrm{Ft}^{3}$ core

$1,360,000$

Volumetric Heat Generation, $\mathrm{MW} / \mathrm{Ft}^{3}$ ball bed

.683

Temperature, ${ }^{\circ} \mathrm{F}$

Reactor Inlet

.915

Reactor Outlet

550

1250

Core Outlet, avg.

Blanket Outlet, avg.

1540

Fuel Element Surface, max.

561

Fuel Element Center, max.

2170

2440

Operating Power Level, MW

Core Contribution*, MW

Blanket Contribution*, MW

337

291.5

45.5

Operating Pressure Level, psia

965

Operating Helium Flow, lbs/hr.

$1,343,000$

Core Pressure Drop, psi

15.5

*At equilibrium conditions, after 10 core lifetimes. 
Section 3.0

Reactor Fuel

3.1 Fuel Elements $\ldots \ldots \ldots \ldots \ldots \ldots \ldots \ldots \ldots \ldots \ldots \ldots \ldots \ldots \ldots$ 3-1

3. 1. 1 Manufacturing Methods ..................... 3-1

3. 1.2 Experimental Work ........................

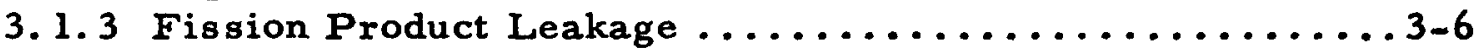

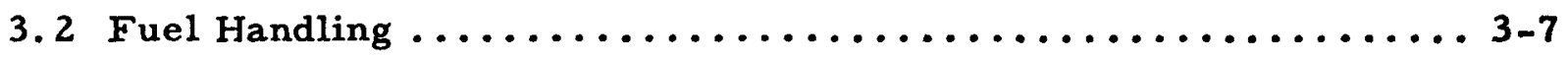

3.3 Fuel Reprocessing $\ldots \ldots \ldots \ldots \ldots \ldots \ldots \ldots \ldots \ldots \ldots \ldots \ldots$. 11 


\subsection{Reactor Fue1}

\subsection{Fuel Elements}

Fuel and blanket elements as used in this reactor system are spheres of graphite containing oxides of uranium and thorium, either separately or mixed, depending upon the location. Requirements for these two types of elements are listed below:

Location

Diameter, in.

Number required

Graphite density, $\mathrm{gm} / \mathrm{cc}$

Composition

Oxide loading, wt.\%

$\mathrm{UO}_{2}$ loading, gms

$\mathrm{ThO}_{2}$ loading, gms
Core

$1-1 / 2$

228,600

1.7

$\mathrm{UO}_{2}+\mathrm{ThO}_{2}$

10

0.449

4.94
Blanket

$3 / 4$

$2,370,000$

1.7

$\mathrm{ThO}_{2}$

50

5.66

\subsubsection{Manufacturing Methods}

Three methods of manufacture are available for fabrication of the fuel elements for this reactor, namely impregnation of graphite spheres, admixture of fuel in graphite before molding the sphere, and lumping the fuel in a small sphere which is then jacketed with graphite during molding. The relative advantages of these fuel types are shown qualitatively in Figure 3.1-1.

Impregnation of graphite with uranium has been done on a laboratory scale by Argonne National Laboratory, North American Aviation, and the Los Alamos Scientific Laboratory. In general the process consists of impregnating preheated spheres $\left(150^{\circ} \mathrm{C}\right)$ in a boiling uranyl nitrate solution, air drying, followed by baking at $275^{\circ} \mathrm{C}$ to drive off $\mathrm{NO}_{2}$, and finally baking at about $800^{\circ} \mathrm{C}$ in a helium atmosphere. When graphite is impregnated with uranyl nitrate in this manner, the uranium is present as $\mathrm{UO}_{2}$.

Figure 3.1-2 presents the maximum theoretical loading of uranium in graphite of various densities together with an estimate of a probable maximum loading by a single impregnation. The loading required in the 125 eMW-PBR is $10 \%$ by weight of $\mathrm{UO}_{2}$ and $\mathrm{ThO}_{2}$. 
Figure 3.1-1

Relative Advantages of Fuel Element Manufacturing Methods

TYPE

IMPREGNATED ADMIXTURE LUMPED
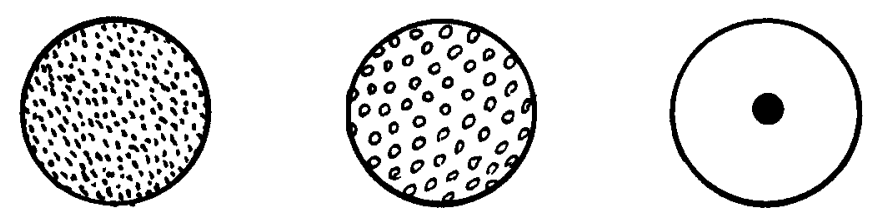

Retention of

2

2

1

fission products

Structural integrity

3

under irradiation

$\%$ weight fissile material

3

2

1

Mechanical strength

1

2

1

Development of manu -

1

1

2

facturing technique

Economy of manufacture

1

2

3

Ease of remote fabrication

1

2

2 
FIGURE 3.1-2

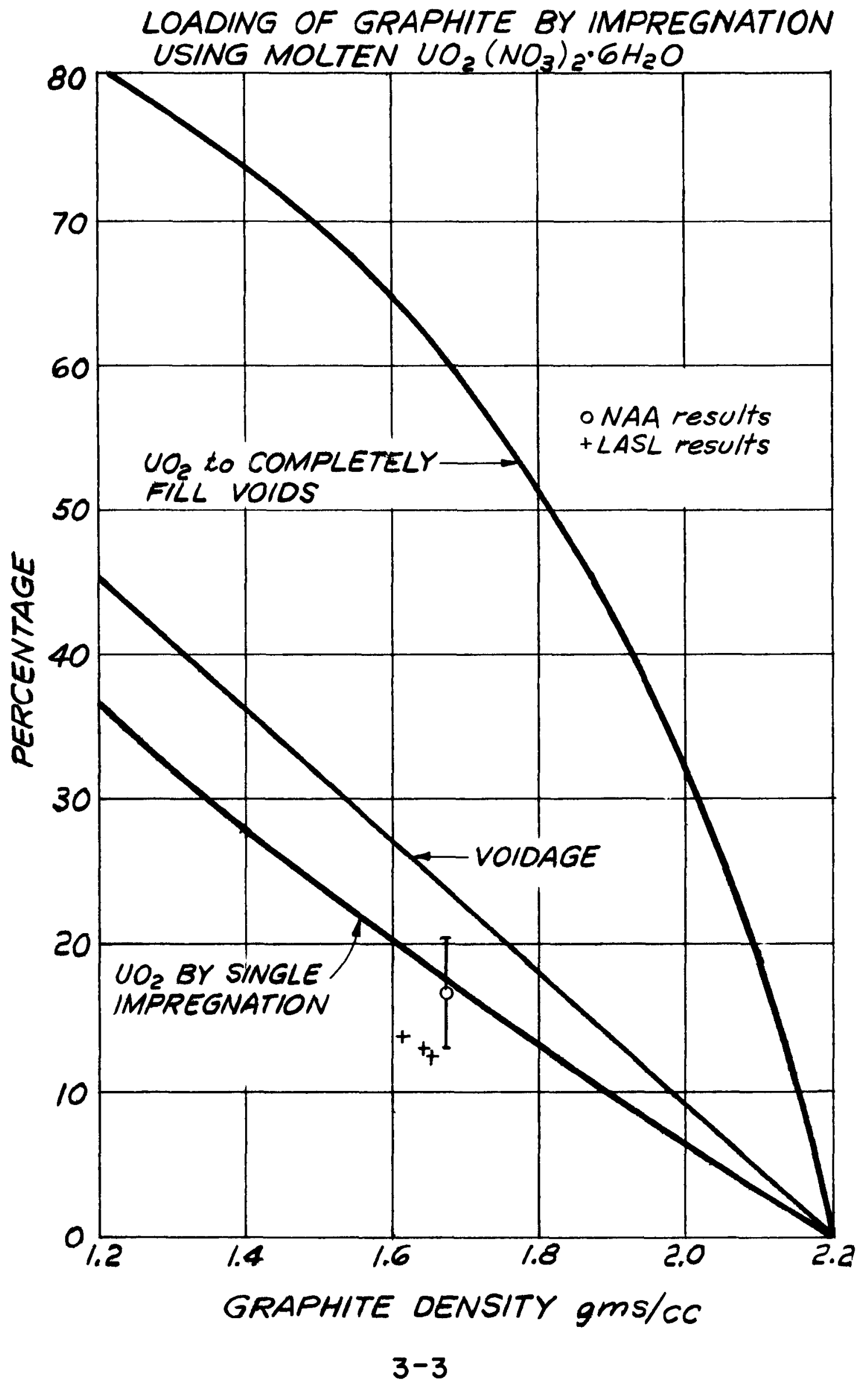


In a fuel element which is to contain both thoria and urania, the impregnating solution could contain thorium nitrate and uranium nitrate in the proper proportions and Figure 3.1-2 would apply to the solution of both $T h \& U$. Such a technique would be adequate for our needs. If under some circumstances, it was desirable to have more than about 10 wt $\%$ total oxide, the $\mathrm{ThO}_{2}$ could be added at the time the ball was molded (using unirradiated thoria, which would present no material handling problems) and the uranium added later by impregnation. In this way the limits implied in Figure 3.1-2 would now apply to the urania only.

The basic advantage of the use of this impregnation technique in the manufacture of PBR fuel elements is its adaptibility to remote fabrication, thus making it possible to fabricate elements with solutions which have had only a small decontamination factor during fuel reprocessing.

Fabrication of fuel balls by dispersion of $\mathrm{UO}_{2}$ grains in a graphite matrix has been done on a laboratory scale by Battelle Memorial Institute and National Carbon. In general the process is to blend $\mathrm{UO}_{2}$ and graphite flour with a binder and mold to size under pressure. After molding the ball is baked to set, or in some cases to graphitize, the binder. By varying the grain size of the graphite flours, the rate and temperature of the baking process, the amount and kind of binder, etc., an almost infinite variety of graphite grades with widely different properties can be produced. If the binder is graphitized, the uranium will be in the form of $\mathrm{UC}_{2}$ rather than $\mathrm{UO}_{2}$.

Loadings as high as $50 \%$ metal oxide in graphite are possible by this method of manufacture. Two phase ceramic bodies of this type where no chemical bond exists between phases tend to have lower values of physical properties such as tensile strength and thermal conductivity. However, their resistance to radiation damage is improved.

Impregnated graphite spheres have been procured with weight loadings ranging from 0.55 to $10 \%$ urania. Molded graphite spheres made by the admixture method have been procured containing $3 \%$ urania and also $3 \%$ urania plus $27 \%$ thoria. A limited amount of physical testing has been done with these sample elements.

A third method of manufacture involves preshaping the fuel into a bulk form and adding the graphite around it. Thus the graphite becomes 
the jacket in the same manner as uranium slugs are canned in aluminum or zirconium. This method, which permits fuel loadings of a high weight percent, is under study by Minnesota Mining and Manufacturing Co. This method of manufacture results in fuel elements with some obvious advantages and disadvantages. On the plus side, the irradiation damage to the graphite will most certainly be reduced, and the fission product leakage might be reduced. The thermal conductivity of the graphite is not affected by the presence of the lump of $\mathrm{UO}_{2}$ as it would be if large percentages of $\mathrm{UO}_{2}$ were mixed with the graphite during molding. On the other hand, the conductivity of the lump of urania is very low and limits the size of such elements. For the $125 \mathrm{eMW}$ $\mathrm{PBR}$, the $\mathrm{UO}_{2}$ lump would be only about $2 \mathrm{~mm}$ in diameter and therefore problems resulting from the low conductivity of the $\mathrm{UO}_{2}$ would be almost wholely absent.

There are several ways in which a "lumped" fuel element can be manufactured: a pellet of $\mathrm{ThO}_{2}$ and $\mathrm{UO}_{2}$ can be encased in a graphite shell; a mixture of carbon, $\mathrm{ThO}_{2}$ and $\mathrm{UO}_{2}$ can be encased in a graphite shell; a mixture of carbon and $\mathrm{ThO}_{2}$ can be encased in a $\mathrm{UO}_{2}$ shell and then a graphite shell; or a pellet of $\mathrm{UO}_{2}$ can be encased in a carbon $\mathrm{ThO}_{2}$ shell and then a graphite shell. Of these, a carbon $\mathrm{ThO}_{2}$ matrix encased in a $\mathrm{UO}_{2}$ shell and then a graphite shell is the best from a thermal and nuclear standpoint. Fabrication of these lumped fuel elements is in all probability more expensive than either molding or impregnation.

The $3 \mathrm{M} \mathrm{Co}$. is also developing a coating for graphite which it is expected will give the fuel element an impervious skin. This coating, known as "3M Ceramic S", in laboratory tests, has protected graphite against oxidation in air for 200 hours at temperatures to $1400^{\circ} \mathrm{C}$. Such a coating increases abrasion resistance, increases corrosion resistance, and provides some degree of fission product retention. This special ceramic coating can be applied to fuel elements made by any of the three procedures described above.

\section{1 .2 Experimental Work}

In order to confirm the feasibility of loading and unloading the reactor by pouring, drop and impact tests were conducted on graphite balls, graphite balls containing $\mathrm{UO}_{2}$ and graphite balls containing $\mathrm{UO}_{2}$ and $\mathrm{ThO}_{2}$. Drop tests were terminated after repeated drops from a height of 20 feet on a steel plate showed no damage or plastic deformation of any kind. Balls were then subjected to an impact test by dropping a 
five pound weight on them from various heights. Results are as follows :

Type AGOT plain graphite machined ball, 1-7/16" diameter, weight 0.105 lbs: broke with a brittle fracture at 25 foot pounds, free fall equivalent 238 feet.

Type AUC plain graphite machined ball, 1-7/16" diameter, weight 0.0955 lbs: broke with a brittle fracture at 10 foot pounds, free fall equivalent 105 feet.

Molded graphite ball containing $3 \% \mathrm{U}, 1.41^{\prime \prime}$ diameter, weight $0.0878 \mathrm{lbs}:$ broke with a brittle fracture at 10 foot pounds, free fall equivalent 114 feet.

Molded graphite ball containing $3 \% \mathrm{U}+27 \% \mathrm{Th}, 1.41$ " diameter, weight $0.111 \mathrm{lbs}$ : broke with a brittle fracture at 10 foot pounds, free fall equivalent 90 feet.

From this data it is reasonable to assume that there will be no breakage of sound fuel elements in loading and unloading the reactor by gravity as the free fall distance in the final design is of the order of $25 \mathrm{ft}$. at the most. In production, fuel elements can be inspected by a simple bouncing technique separating the ones having incipient cracks or internal voids, This will eliminate the possibility. of fracturing such faulty fuel elements during loading.

Another question that has been investigated by others is the stability of graphite parts when exposed to a high velocity jet of hot helium. Tests of this nature have been conducted by North American Aviation and reported in NAA-S R-77. Tests with helium flowing through a nozzle at a throat velocity of $9000 \mathrm{ft} / \mathrm{sec}$. and a temperature of $2000^{\circ} \mathrm{C}$, impinging on a specimen of graphite heated to $2000^{\circ} \mathrm{C}$, disclosed that for about 24 hours the machined graphite surface was subject to an initial erosion process in which the loosely held particles, smeared into the surface during machining were blown free. Once this process was completed, no further erosion was apparent. Since the PBR operating conditions are appreciably lower than those of these tests it can be concluded that there should be no question regarding the stability of graphite parts in the helium stream.

\subsubsection{Fission Product Leakage}

During reactor operation, volatile fission products will diffuse from the fuel elements and circulate with the gas stream. The quantity of 
fission products which are released will depend on the method of manufacture of the fuel element and on the temperature of the reactor core. Use of a coating such as "3M Ceramic S" will facilitate the retention of fission products within the fuel element, however the performance of this coating is as yet unproven. It has been assumed therefore that all of the fission products volatile below $2500^{\circ} \mathrm{F}$ will be released to the gas stream. This assumption affords a conservative basis for the primary system shield design. This assumption is quite conservative for three reasons:

1. Only the fuel element center temperature ever reaches $2500^{\circ} \mathrm{F}$.

2. Only part of the fuel elements are in the hot zone in the reactor. Fully $98 \%$ of the fuel elements will have surface temperatures significantly below $2000^{\circ} \mathrm{F}$.

3. Some of the gaseous fission products have such short half lives that it is improbable that they could diffuse out of the fuel element before they decay to non-volatile daughters.

The principal fission products which require our attention are the halogens, $\mathrm{Br}$ and $\mathrm{I}$, and their rare gas daughters, $\mathrm{Kr}$ and $\mathrm{Xe}$. Figure 3.1-3 indicates the total curies of activity of each gaseous element which would exist in the primary system based on a reactor operating at $350 \mathrm{MW}$ heat output. The graph shows the growth of activity over a 5 months operating cycle as well as the decay following shutdown. Gases with a half life of $1 \mathrm{sec}$. or less have been neglected since they would decay before they could diffuse out of the spheres. Also Xe-135 has been neglected since its equilibrium level and its growth after reactor shutdown depend on the operating flux level of the reactor. It can be seen that the activity due to $\mathrm{Br}$ decays so rapidly after shutdown as to present no problems. Facilities have been provided however for precipitation of the iodine and adsorption of the xenon and krypton.

\subsection{Fuel Handling}

The core lifetime of the reference design is 100 days at full power. It is intended that the entire core be replaced at one time. The blanket elements, on the other hand, can be irradiated for several years. It has been shown in Section 3, Part II that it is advantageous to unload $1 / 10$ of the blanket each time the core is changed. Fuel handling therefore involves removing all the core elements and some of the blanket elements at the end of each core life. 


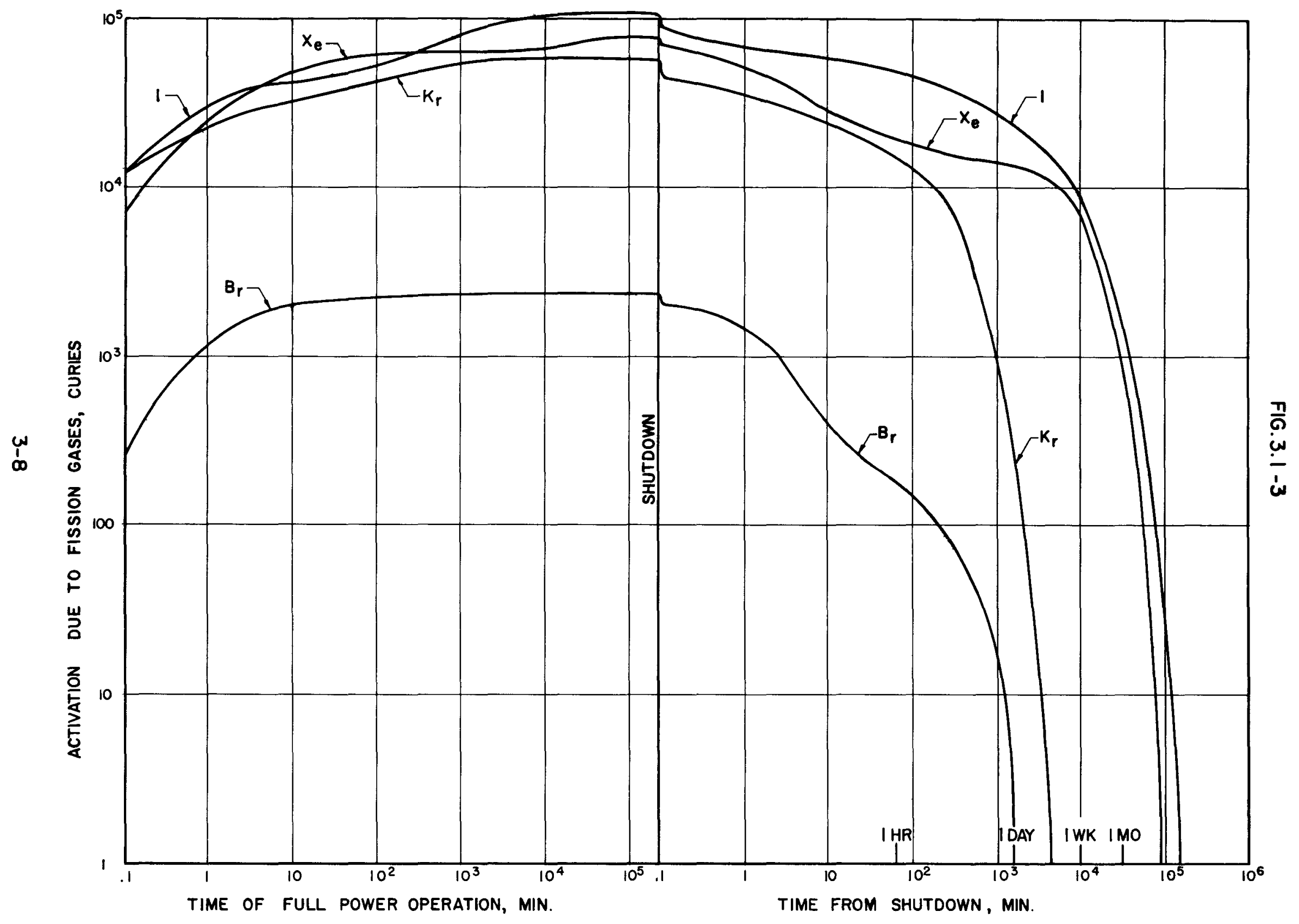

مَ 
As shown in Drawing \#1963-B-19 fuel elements and blanket elements all drain through a common outlet at the base of the reactor. A detailed description of the bottom support structure of the core as well as of the unloading mechanism can be found in Section 4.2.3 of this report. Experimental work on scale models has demonstrated that the central core channel drains first, followed by the six outer core channels, and finally the blanket. During the period when the central core hole is draining, there is no ball flow from either the outer core holes or from the blanket. This selectivity also prevails while the outer core regions are draining.

The simplest unloading procedure consists of drawing the entire core and blanket at one time. Any procedure which requires that less than all the reactor be drained at once introduces the need for a device capable of giving a positive measure of the amount of fuel unloaded. The procedure selected involves unloading some of the fuel into a fuel metering chamber of definite volume. When the chamber is full, the fuel balls simply stop flowing after which the ball discharge valve can be closed.

The core of this reactor contains roughly $383 \mathrm{ft}^{3}$ of fuel bed. An unloading chamber of $35 \mathrm{ft}^{3}$ capacity is a convenient size and with it the core can be unloaded in 11 increments. If one tenth of the blanket is to be removed each time the core is changed, then a total of 13 increments will be removed, eleven increments of which represent the core and two represent the blanket. In this way the blanket will have been changed once when the core is changed 10 times.

Loading is done using canisters containing a definite volume of fuel elements. The size of the canisters must be small enough so that only a small fraction of the core can be added at any one time. In this way the change in reactivity during charging can be followed and the dangers associated with accidental overcharging are eliminated.

After each refueling, blanket elements are loaded using a separate canister in six increments of $8.3 \mathrm{ft}^{3}$ each in order to insure uniform distribution of the blanket elements around the core.

In loading, the blanket elements are loaded first. This causes the grate structure at the bottom of the reactor to be completely covered with a layer of blanket balls approximately one foot deep. The 
core fuel elements are loaded next. The reactor core is therefore blanketed on the bottom as well as on the sides. When the core is drained, that portion of the blanket resting on the bottom grate immediately below the core, is drained as well. By presetting the bottom blanket thickness, just the right amount of blanket balls are removed with each core change. When discharge of the core is complete, blanket balls from the side run down over the bottom grate to a fixed depth as at initial startup. The fresh core and blanket charge is then added and the reactor is ready for another cycle.

Several alternate fuel loading schedules are possible and have sorne merit. For example, advantage could be taken of the core flux distribution by permitting longer burnup of the fuel in the outer core holes and consequently lower reprocessing costs. At the end of the first core lifetime, only the central channel would be reloaded. At the end of the next core lifetime, the entire core could be reloaded and the cycle repeated.

Perhaps the most desirable loading schedule for the PBR is one involving addition and withdrawal of a small amount of fuel on a day to day basis, i.e. essentially continuous refueling. This refueling schedule permits operation at a $\mathrm{K}_{\text {eff }}$ slightly greater than 1.0 and there is no need for large numbers of control rods to poison out the large initial excess reactivity needed to give a finite lifetime. The smaller number of control rods required in a continuously refueled core can be clustered at the center of the reactor and the seven fuel holes can be combined to give one large fuel region. This is essential if day to day refueling is to be practiced. Spent fuel would be withdrawn from the bottom of the core and fresh fuel added at the top of the core. At equilibrium, this fuel distribution would tend to peak the axial power distribution towards the top of the core. This is desirable from the thermal standpoint since the helium inlet is also at the top of the core. In order for day to day refueling to be economically justified, it must be associated with a continuous reprocessing system on the site rather than to depend on the AEC multipurpose plant.

Certain precautions must be taken during loading and unloading to prevent the inleakage of air or moisture or the outleakage of fission product gases. The detailed loading procedure would be as follows:

1. The loading canister is filled with fuel or blanket elements in the Fuel Handling Room, evacuated and purged with helium.

2. The canister is brought over the appropriate loading tube by the plant crane, lowered into position, and made fast to the loading 
tube by means of a breech-type closure.

3. The pipe length between the canister and the shut-off valve on the loading tube is evacuated and purged with helium.

4. The valves on the canister and on the loading tube are opened wide allowing all the elements in the canister to flow into the reactor.

5. The valve in the loading tube is closed and the pipe and canister are evacuated through the activated charcoal traps to the helium storage system. The canister is then purged with helium, re-evacuated, repurged and so on until the fission gases which diffused into the canister while connected to the reactor will have been reduced to tolerance levels.

6. The canister is disconnected and the open end of the loading tube plugged.

At the discharge end, the unloading canister is an integral part of the piping system. It can be isolated above and below by gas-tight block valves. At the lower end of this canister, a pipe leads off to the fuel handling room. Fuel unloading casks can be attached to the discharge tube at this end, to receive spent fuel for storage and decay prior to being shipped to the reprocessing plant. The same precautions against inleakage of air or moisture and outleakage of gaseous fission products must be taken at this end of the system as have been described for the charging end.

There are several methods of cooling the spent fuel elements. The spent fuel casks can be connected to a closed gas loop cooling system wherein the heat is transferred to a water cooled exchanger. Another alternative would be to store the casks in a water bath so that decay heat could be transferred through the walls of the container. Suitable builtin poisons would be required to prevent criticality. Rather high temperature could be permitted in the elements at the center of the cask to offset the poor thermal conductivity of the ball bed. A third alternative would be to quench the spent fuel elements in an inert gas stream and pour them into a cask filled with water. A reflux condenser would return condensed water vapor by natural circulation thus requiring no coolant pump.

\section{3 Fuel Reprocessing}

In the original conception and early development of this reactor system, it was intended that a fuel reprocessing plant be designed expressly for these graphite fuel elements. Such a single purpose plant designed to take advantage 
of the relatively simple head end treatment associated with these elements should result in significant savings in fuel reprocessing costs. However, in order that our results be more readily compared with those of others we have based our fuel economics on the AEC Reference Fuel Processing Plant (WASH 743). This is a multipurpose plant intended to service a wide variety of fuel elements. It therefore involves a number of parallel dissolution systems with a common extraction system.

Recognizing that the fuel reprocessing costs so obtained are only relative, we believe them to reflect unfavorably on this design since they fail to take cognizance of the unique character of this fuel.

The AEC Multipurpose Plant was developed for stainless steel clad or zircoloy clad elements and is therefore provided with rather elaborate head end facilities. These include 1) A Mechanical Treatment Section provided with under water sawing equipment to cut off the ends of fuel jackets, 2) Two Zircex dissolver systems for dissolution of Zirconium clad fuel elements, 3) Two Darex dissolver systems for dissolution of stainless steel in aqua regia, 4) $\mathrm{An}_{2} \mathrm{SO}_{4}-\mathrm{HF}-\mathrm{HNO}_{3}$ dissolver system for dissolution of fuel elements containing both $\mathrm{Z} r$ and stainless steel, and 5) All the associated waste and recovery systems.

By contrast the head end treatment of the PBR elements require only coarse grinding followed by $\mathrm{HNO}_{3}$ leaching or incineration of the graphite, and finally dissolution of the ash in $\mathrm{HNO}_{3}$. Thus reprocessing of graphite elements would permit elimination of the mechanical treatment and $\mathrm{HCl}$ distillation completely. The dissolution step would consist of a simple nitric acid leaching operation and it is to be expected that the cost of this step and its associated waste problems would be greatly reduced.

The liquid-liquid extraction facilities of the multipurpose plant include four extraction cycles which carry the products through to purified uranium and thorium streams. Presumably the decontamination factor is high enough to permit direct handling of the finished product during manufacture of new fuel charges. If the fuel is reconstituted by impregnation, which is amenable to remote operation, a decontamination factor of about $10^{2}$ would be adequate in which case the extraction facilities of the multipurpose plant are much more than adequate.

WASH 743 contains the statement "Although this standard charge is applicable to all the fuels considered herein, if a fuel type involves a significantly higher or lower portion of the capital in the plant or higher 
or lower operating or waste disposal costs, the standard charge may be modified accordingly". We believe that this statement is appropriately applied to the graphite uranium fuel type described in this report. However, having no objective way of adjusting these costs, we have elected to us the daily operating charges stipulated inWASH 743, realizing of course that this would result in conservative fuel costs.

The detailed economic analysis of the fuel reprocessing is presented in Section 3.0 of Part II. 


\section{Section 4.0}

\section{System Design}

4.1 Plant Design and Arrangement $\ldots \ldots \ldots \ldots \ldots \ldots \ldots \ldots, \frac{\text { Page }}{4-1}$

4.2 Reactor ............................. $4-3$

4.2.1 Mechanical Design .................... 4-3

4.2.2 Control Rods and Drive Mechanisms ........... 4-5

4.2.3 Loading \& Unloading Mechanism .............. 4-6

4.3 Primary System $\ldots \ldots \ldots \ldots \ldots \ldots \ldots \ldots \ldots \ldots \ldots \ldots \ldots 4,7$

4.3.1 Steam Generators .................. 4-7

4.3.2 Helium Compressors ...................4-10

4.3 .3 Piping \& Valves $\ldots \ldots \ldots \ldots \ldots \ldots \ldots \ldots \ldots \ldots \ldots, 4-11$

4.3.4 Gas Handling System ................... $4-12$

4.3.5 Emergency Procedures ................. 4-13

4.3.6 Clean-up and Maintenance ................ 4-15

4.4 Shielding and Containment $\ldots \ldots \ldots \ldots \ldots \ldots \ldots \ldots \ldots \ldots, 21$

4.4.1 Shield Design ......................,4-21

4.4.2 Containment Vessel ................... 4-22

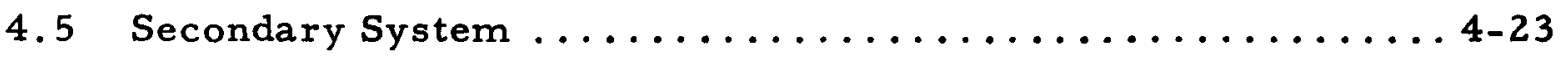

4.5 .1 Heat Balance .........................

4.5.2 Turbine Generator ....................4-24

4.5 .3 Auxiliary Equipment ....................

4.5.4 Electrical Equipment ................. 4-26

4.6 Control and Instrumentation .................. 4-29

4.6.1 Reactor System Instrumentation ............ 4-30

4.6.2 Steam Plant Instrumentation .............. 4-30 


\section{0 System Design}

\section{1 Plant Design and Arrangement}

Since the site for the 125 eMW Pebble Bed Reactor Steam Power Plant has not been defined, it is necessary to make some basic assumptions in order to establish a conceptual design for the facility. This power plant layout is based on a location adjacent to a river, and within a property area consisting of approximately 350 acres of land. A plot plan for the 25 acres of developed area for the site is shown on Drawing 1963-B-10. The size of the generating unit dictates that the site be within 50 miles of a rather large population center. It is also assumed that the station will be part of an electric generating utility which is interconnected with other utility systems.

Site access is furnished by both a paved road connected to a highway and a railroad siding. Property improvements include sanitary sewage, disposal system, yard drainage, outside fire protection, water supply system, elevated water tank, fencing, guard house and parking areas. An amount has been included in the cost estimate for an aqueous waste storage and disposal facility, although no design effort has been spent on this feature.

The plant structures consist of a containment vessel to house the primary system and a conventional structure to house the secondary system. Detailed layouts of the plant arrangement are shown on Drawings 1963-B-11, 12, 13 and 14 .

The containment vessel, which is located 75 feet from the turbine generator building houses all equipment containing high pressure helium that could become contaminated because of radioactivity in the helium stream. This includes the reactor, steam generators, circulating blowers, transfer compressors, helium storage bottles and certain gas clean-up equipment. A circular crane for handling equipment during construction and subsequent operation of the plant is provided.

The reactor service building, shown on Drawings 1963-B-11 and 12 , is located adjacent to the containment vessel with access through a lock. On grade level are the office and laboratory space, a change 
room, a room for equipment that will handle decontaminating liquids, and the fresh fuel storage and handling area. When reloading the reactor, fuel casks a re taken into the containment vessel via the access hatch. As shown on Drawing 1963-B-11, the first level below grade (Elev. - 18'-0'') of the reactor service building contains equipment that will have some radioactivity, such as equipment for decontaminating all low pressure off gas streams prior to discharging them to the stack. The hot machine shop is also located at this level. Spent fuel elements from the reactor are dishcarged into equipment located at Elev. -40'-0'". The fuel elements will be stored in this cell until they are shipped off the site for reprocessing. An access shaft to Elev. $-40^{\prime}-0^{\prime \prime}$ is provided to permit transfer of the fuel element casks from the storage cell to the railroad spur.

The turbine generator building is designed as an indoor type with enclosed turbine hall and auxiliary bay. It is assumed that the maximum and minimum river levels are such as to permit its construction on grade. Consequently, the turbine generator pedestal and building structure are supported on a reinforced concrete slab.

Space is provided in this building for plant administrative offices and the main control room for both the primary and secondary loops. Specific areas of both the turbine generator and reactor service building will be air conditioned.

Circulating water is furnished through an intake tunnel and discharged into the river at a point sufficiently downstream of the intake to prevent recirculation. Ample space is available for cooling towers if this feature is needed because of periodic insufficient water flow in the river. 


\subsection{Reactor}

The reactor for the $125 \mathrm{eMW}-\mathrm{PBR}$ is shown in drawing 1963-B-19. It is of all graphite construction with the core elements contained in seven vertical channels and the blanket elements contained in an annular ring formed by the core channel structure and the thermal shield. Heat is removed from the reactor by helium flowing downward through the core and blanket in parallel. Reactor control is effected by twelve vertical top driven rods located in the core channel structure around the central channel. Top reflection is achieved by a top graphite structure forming seven holes over the seven fuel channels, each provided with a heavy graphite cap supported by the local fuel loading tube. Bottom reflection is achieved by a grate structure, plenum chamber lining and a conoid hood forming the gate for the unloading tube. Helium enters and leaves the reactor through three co-axial pipes at the bottom of the vessel. Fuel is loaded through multiple loading tubes at the top of the reactor vessel and unloaded through a single discharge valve at the bottom of the reactor vessel.

\subsubsection{Mechanical Design}

As shown in drawing 1963-B-19, the fuel elements are located in 7 chambers |(a central chamber and six outer chambers). The chambers are formed by six graphite cylinders which are bored axially with an obround hole, $31^{\prime \prime} \times 37^{\prime \prime}$. Each cylinder has a basic outside diameter of 44-1/2". They are machined flat on three adjacent sides so that the six cylinders can be nested together to form the central chamber which is a 39" hexagon. Adjacent blocks are keyed together to form an integral structure of graphite. Longitudinal holes for control rods are provided in the wall of each cylinder which forms a side of the central hexagonal chamber. The blanket elements are located in an annulus formed by the graphite core cylinders and the thermal shield.

The graphite core cylinders are supported on graphite grates, which support both the fuel elements and the blanket elements while permitting the reactor coolant to flow through into a plenum chamber below the grates. Each of the twelve grate sections has 2 " $\times 12^{\prime \prime}$ deep side beams which also support the graphite core cylinders. The grating is formed by $3 / 4$ " thick $\times 12$ " deep graphite plates, separated by spacers and joined with graphite bolts. The twelve grate sections are bonded and keyed together to form a conical floor which is pitched at a $15^{\circ}$ angle towards the centrally located fuel discharge valve. 
Appropriate openings are cut in the bottoms of the six graphite core cylinders to permit the fuel and blanket balls to flow down along the grate to the discharge valve. The six graphite core cylinders are supported and centered on the conical grate structure which is in turn supported by an innner and an outer graphite cylinder resting on a steel bed plate attached to the bottom head of the vessel. The bed plate incorporates the three $16^{\prime \prime}$ reactor gas outlet lines which are co-axial with the three 24 " inlet nozzles. The incoming helium flows upward through the annulus formed by the pressure vessel wall and the thermal shield. The gas then turns through openings in the the rmal shield and flows downward through the core and blanket in parallel. Graphite reflector plugs are attached to fuel loading tubes over each of the seven fuel chambers.

All permanent graphite parts are treated with an impervious graphite coating, such as siliconized silicon carbide, to prevent irreparable damage which might result from a steam leak into the primary system during operation.

The reactor pressure vessel is cylindrical in shape having hemispherical heads at each end. As a minimum requirement, the vessel will be designed, fabricated, inspected and tested in accordance with the ASME Code for Unfired Pressure Vessels.

The lower hemispherical head supports the graphite core structure and has one nozzle attachment for the unloading tube and three nozzle attachments for the concentric pipes which constitute the inlet and outlet gas passages. All nozzles will have an integral reinforcing to comply with Code Case 1234 which refers to pressure vessels subject to gamma radiation.

The upper hemispherical head contains seven (2"pipe) penetrations for the core loading tubes and six (1-1/2" pipe) penetrations for the blanket loading tubes. An access hatch is provided in the upper hemispherical head for installation of the core structure. This hatch is permanently welded into place after the core is installed. This hatch provides mounting pads for the twelve control rod drives. To insure proper alignment between the control rod mounting pads and the control rodpassages in the core structure, a final field machining operation is necessary. The cylindrical portion of the vessel incorporates the vessel mounting brackets as well as internal supports for the upper thermal shield. 
Because of the fact that helium is an inert gas, the material and fabrication problems usually encountered in other reactor vessel designs are not present. In addition, since the helium entering the vessel is first directed through an annular passage formed by the thermal shield and the vessel wall, conventional materials can be used in spite of the $1250^{\circ} \mathrm{F}$ reactor outlet temperature.

The vessel plate material is SA-212B (carbon steel) and nozzle flanges are forged from SA-105 Grade II (carbon steel). No special internal surface finish is required. Vessel design requirements are as follows:

$\begin{array}{ll}\text { Design pressure } & 1100 \mathrm{psi} \\ \text { Design temperature } & 650^{\circ} \mathrm{F} \\ \text { Test pressure } & 1650 \mathrm{psi} \\ \text { Test temperature } & \text { prevailing ambient } \\ \text { Code requirements } & \text { ASME-UPV Code (1956) } \\ \text { Inside diameter } & 12^{\prime} 10^{\prime \prime} \\ \text { Overall length } & 21^{\prime} 0^{\prime \prime} \\ \text { Cylindrical wall thickness } & 5-1 / 2^{\prime \prime} \\ \text { Hemispherical head thickness } & 2-3 / 4^{\prime \prime} \\ \text { Weight, empty } & 142,000 \mathrm{lbs} . \\ \text { Thermal shield thickness } & 2^{\prime \prime}\end{array}$

\subsubsection{Control Rods and Drive Mechanisms}

Twelve control rods $4 "$ in diameter and having a 1/4" wall thickness will be positioned in the core as shown in drawing 1963-B-19. The active absorber section of these control rods will be fabricated from Haynes "25" alloy. Each section will be 99" long weighing approximately 83 pounds. The rods are cooled by helium flowing inside and outside of the absorber section.

Drawing AEL-271 shows a cross-section of the proposed control rod drive mechanism. An electric gearmotor drives the control rod via a magnetic clutch, an over running clutch, a labyrinth seal, and Oldham type coupling for minimizing misalignment, a pinion, and a rack which is attached to the control rod. The magnetic clutch is disengaged by a scram signal permitting the 175 pound rod to fall by gravity. Deceleration is provided by a set of two pistons and two springs. The overrunning clutch will make the full torque of the drive motor available if a rod should stick for any reason. Rod position signals are transmitted by limit switches and a synchro-geared to the pinion. 
The gearmotor is mounted inside a vessel which is flanged to the drive housing making a hermetically-sealed assembly. When the gearmotor and its vessel are withdrawn, the labyrinth seal limits leakage for the short time required to seat a special spherical valve on the drive shaft which provides a leak-tight seal. This arrangement permits removal of the drive without completely depressurizing the primary reactor system.

\subsection{Loading and Unloading Mechanism}

The Pebble Bed Reactor is loaded and unloaded by gravity

Loading is effected by pouring balls into the core and blanket regions through tubes. Each of the seven core regions is loaded by individual tubes centrally located over each channel. The blanket region is loaded through six tubes equally spaced around the blanket annulus. These tubes are sized at about 1-1/4 ball diameters and pass through the upper reactor shield and terminate on the floor of, a circular platform built over and around the control rod drives. A gate valve is located at the upper end of each tube just below the platform floor level. When not in use the loading tubes are capped to provide an absolute seal.

Unloading of the reactor is effected through a single unloading valve, centrally located in the conical grate, as shown in drawing 1963-B-19. This valve consists of a refractory tube driven on its vertical axis by a rack and pinion. In the upper position the tube stands above the bottom grate and fuel and blanket elements are prevented from entering by a refractory conoid hood over the unloading tube. Appertures around the battom of this conoid hood allow fuel and blanket balls to rise to the level of the unloading valve in the upper position. As the unloading valve is lowered, the fuel and blanket balls roll over its upper rim and drop through the valve into a metering chamber of fixed size external to the reactor. When this chamber is full, the valve is raised, preventing - further flow of core and/or blanket balls from the reactor. Balls are removed from the chamber by opening a gas-tight valve in the outlet line, permitting balls to flow to spent fuel casks in the fuel handling room in the lower level of the reactor service building. 


\subsection{Primary System}

The primary system consists of three parallel circuits, each rated for $1 / 3$ of the system output. Each circuit includes one steam generator and one compressor. The total volume flow of approximately $60,000 \mathrm{cfm}$ at design conditions, dictated that at least three compressors would be required. Since the resulting size of the steam generating units was within shop fabrication limits, three circuits were selected.

Because of the anticipated reliability of the proposed steam generator design, we have proposed to handle the consequences of a tube failure by suitable operating procedures as discussed in Section 4.3.5. This avoids the additional capital cost and increased pumping power of a secondary gas loop, and the added design complexity of double wall tubes.

Although the primary loop is designed to handle $1200^{\circ} \mathrm{F}$ helium, all pressure containing parts are subject only to the reactor inlet temperature. Cold gas leaving the steam generator first passes over the wall of the vessel. After leaving the compressor, the gas passes through the outer annulus of the double wall pipe run between the steam generator and reactor. Reactor inlet gas passes over the reactor vessel wall before entering the core. This method greatly simplifies the problem of handling high-pressure, high-temperature helium. The only drawback is the slight preheating of the reactor inlet gas which in effect increases the average reactor temperature about $50^{\circ} \mathrm{F}$.

The primary system is installed within the containment vessel. Dwg. 1963-B-8 is a plan view of the containment vessel and the equipment therein and Dwg. 1963-C-7 is a vertical elevation of same. As shown in these drawings, the three major pieces of equipment in the primary loop - the reactor, the compressors, and the steam generators -are all supported at the same elevation. Since all walls are at essentially the same temperature, stresses caused by expansion in the vertical direction are eliminated and the small horizontal expansion in the run between the steam generator and the reactor is readily taken by the pipe bend, thus requiring no expansion joints.

\subsubsection{Steam Generators}

The three steam generators raise a total of $1,056,000$ pounds per hour of $1450 \mathrm{psig} / 1000^{\circ} \mathrm{F}$ steam. This steam is produced from water 
of controlled purity with an inert gas of controlled temperature. There is no piece of equipment in industrial use today operating under similar conditions which makes it difficult to postulate probable failures, although there is ample experience with waste heat exchangers upon which to base the design. Tube failures in waste heat exchangers are caused by thermal stress and stress corrosion. There are no conditions in this design which would result in corrosion stresses. Thermal stresses a re usually caused by mal-distribution of gas flow, deposits on tubes and poor design. Distribution of gas flow can be made ideal, and in a closed system, in which an inert gas is the heat transport fluid there is little if any opportunity for adherent deposits to be formed on tubes. Therefore, the successful operation of such a steam generator is dependent upon good design practices.

Steam generators of this type can be designed as natural circulation, controlled circulation or once-through. Natural circulation is not desirable due to the large volume of water that would be present in the containment vessel. Controlled circulation is a conservative choice since it 'requires a smaller volume of water and is less sensitive to water purity. The once-through type represents the ideal in design, providing problems of flow distribution can be handled in an adequate manner, as it reduces the number of shell penetrations and the volume of water in the containment vessel.

Pending further study of the design requirements we have chosen a controlled circulation steam generator for this design study. Each of the three units has its own steam drum and recirculating pump, and the steam side of the three units a re completely independent within the containment vessel. Automatic block valves are installed just outside the containment vessel in the three steam lines and three feedwater lines which permit isolation of the water side of any one boiler circuit in the event of trouble. The high pressure stored water volume of one boiler circuit is considered in sizing the containment vessel wall.

The general features of the steam generators are shown in Dwg. 1963-B-20. The generator is essentially a tube and shell heat exchanger $81 / 2$ feet in diameter and 35 feet high in which helium flows through the shell side and water through the tube side. Hot helium enters through a 16 in. pipe at the bottom of the vessel and flows upward through a central 16 in. dia. duct to the region above the tube bundle. The helium flow then turns downward and first passes through an activated ball bed to 
remove chemically active fission product that may be present in the helium stream. The hot helium then flows across the tube bundles. The tube bundles are divided into three sections: a superheating section, an evaporating section, and an economizer section. Cold helium leaving the economizer section at the bottom of the unit turns and flows upward through the annulus formed by the pressure vessel wall and the thin stainless steel shroud which surrounds the tube bundles. Thus, the cold helium serves to keep the pressure vessel wall below $650^{\circ} \mathrm{F}$ and permit the use of the maximum allowable stress in designing the vessel. The cold helium leaves the vessel through a $16^{\prime \prime}$ nozzle located at the side of the vessel near the top.

The tube banks are formed by a double layer spiral of bare tubes where the inner diameter of the spiral is large enough to accommodate the central duct. A thermal sleeve is provided at all points where the tubes penetrate the vessel wall. These spiral wound coils will have perfect freedom to accommodate themselves to expansion. They will pass through the vessel wall and be welded to a thermal sleeve about a foot from the hot gas area. At design conditions the steady state pressure difference between steam and gas is $450 \mathrm{psi}$. Since nozzle guide vane control on the circulating blowers is used for load control, this difference exists from $100 \%$ to $50 \%$ load.

All tubes are brought out horizontally from the generator shell and extend for a distance of about $3 \mathrm{ft}$. before entering the various headers. This $3 \mathrm{ft}$. space will be filled with shielding material which will permit direct maintenance on the tubes without having to decontaminate the system. This design is based on the premise that tube sections will not be replaced in event of failure but that the failed section will be blanked off. Failure of tube banks is most likely at start-up and after the generator has operated for about three fourths of its design life. It is uneconomical and illogical to complicate the design to the extent necessary to provide for tube bundle removal under such premise when any tube failures that might occur can be corrected by the relatively simple expedient of plugging tubes.

This specific configuration for the steam generators evolved from a study of integration of plant components to eliminate expansion joints, and was not a part of our original specification to heat exchanger manufacturers. Proposals have been received for both controlled circulation and once-through steam generators which with minor rearrangement of the tube banks can be adopted to this preferred geometry. 
A forced circulation design is illustrated in Babcock \& Wilcox Drawing No. 6626lE. This unit is 8 feet in diameter and $40 \mathrm{feet}$ high. Tubes are bent in serpentine fashion and assembled inside a square shroud in a cylindrical vessel. Flow is through 64 tubes in parallel each 333 feet long. The tubes are studded to increase the overall rate with reference to the bare tube which results in an appreciable reduction in prime surface. While the Babcock \& Wilcox design shows a co-axial gas inlet and outlet at the top end, the design can be modified to meet our desired geometry by separating the tube banks on the vertical centerline to provide for a rectangular hot gas passage through the center of the unit.

\subsubsection{Helium Compressors}

Large high pressure gas circulating equipment is in common use today as evidenced by applications in gas line pumping and certain high pressure chemical processes such as catalytic reforming. A majority of these applications use centrifugal compressors. Design requirements for the 125 eMW-PBR compressors are $20,000 \mathrm{cfm}$ at $12,000 \mathrm{ft}$. head. It is proposed to use a two stage centrifugal compressor directly driven by a $3600 \mathrm{rpm}$ electric motor. Single stage centrifugal compressors can be obtained with heads up to $15,000 \mathrm{ft}$. but requiring speeds up to about $8000 \mathrm{rpm}$. These speeds would require either an electric motor drive with gear reduction, a direct steam turbine drive, or a high frequency direct motor drive. Because of the added complications of these arrangements, a two stage compressor was selected permitting direct connection to a $3600 \mathrm{rpm}$ motor.

The helium compressor is shown on DeLaval Steam Turbine Co. Dwg. J-1194. The machine is of barrel type construction with the whecl assemblies contained in a forged steel cylindrical sheel. Bearing mounts are attached to the flanged head assemblies at each end of the shell. Suction and discharge nozzles will be horizontal and on opposite sides of the shell, as shown in Dwg. 1963-C-7. In order to accommodate the $500^{\circ} \mathrm{F}$ operating temperature, the compressor will be centerlinemounted and the bearings and seal assemblies will be oil cooled. Leakage of gas from the driveshaft end is prevented by a carbon ring face seal, backed by a lubricant at a pressure head greater than the head of gas being pumped. A fresh helium stream is injected at the center of a double labyrinth located inside the face seal. This sweep helium leaks both into the system and into the seal oil collection chamber. The only gas that can escape from the system is that which dissolved in the seal lubricant which 
will be separated and returned to the system by the oil purification system located in the reactor service building.

The compressor will be driven by a $3500 \mathrm{HP}, 4160 \mathrm{v}, 3600 \mathrm{rpm}$ constant speed motor. In order to accommodate changes in reactor power the gas flow rate can be varied by adjustable prewhirl vanes at the compressor inlet. A standy motor is directly connected to the outboard end of each of the three main drive motors. These motors are rated at $30 \mathrm{HP}, 480 \mathrm{v}$., $450 \mathrm{rpm}$ and will be supplied by the standby power' source to provide emergency cooling for the reactor.

Removable high density shielding will be installed between the compressor and the motor to prevent radiation damage to the motor windings. Since the bearing and seal lubricant is continuously recirculated, it can be filtered and replaced as required to allow for radiation damage.

\subsubsection{Piping and Valves}

The piping layout for the primary system is shown in Dwg. 1963-C-7. The pipe run between the reactor and the boiler is a double wall pipe. The $1250^{\circ} \mathrm{F}$ reactor outlet gas flows in the inner pipe which is stainless steel (SA312, Gr. TP316) 16 in. OD by $1 / 4^{\prime \prime}$ wall. The outer pipe is $24^{\prime \prime}$ dia. Since $500^{\circ} \mathrm{F}$ helium returning to the reactor flows through the annulus, this pipe can be carbon steel (SA106, Gr. B) and permit the use of Sched. 60 pipe having a $0.968^{\prime \prime}$ nominal wall. The pipe run from the compressor discharge to the double wall pipe junction handles $500^{\circ} \mathrm{F}$ helium. It will be SA106, Gr. B, 18" Sched. 60 having a $0.75 "$ nominal wall thickness. All welded construction will be used throughout the primary system, including the nozzle attachments on the reactor vessel, boilers and blowers.

Two dual sets of valves will be installed in each of the primary circuits-one set at the compressor discharge and one set at the boiler inlet. These valves will serve two functions. The compressor discharge valves will shut if one compressor fails to operate, thus preventing backflow through that circuit while the other two circuits continue to function. Also, both sets of valves can be used to isolate a circuit whenever maintenance is required during a reactor shutdown.

Ideally, a primary reactor loop should have no valves in order to avoid additional sources of potential trouble. Instead, we have attempted 
to specify a system where valve requirements are at a minimum. During operation of the reactor, no valve action is required and during an emergency condition or during a maintenance period, the valves are not required to be leaktight across the seat. We have selected butterfly valves for the present system, because they offer a minimum resistance to flow and require a short travel actuator which can be bellows sealed to prevent stem leakage. These valves will be stress sealed butterfly valves similar to those made by the $W$. S. Rockwell Co. These valves have an annular ring which compresses against the outer edge of the butterfly when it is in the closed position which greatly reduces leakage. Valves are installed in pairs so that during a period when either the blower or boiler is open for maintenance, the space between the butterfly valve can be pressurized with clean gas to prevent the escape of radioactivity out of or the introduction of air into the rest of the system.

\subsubsection{Gas Handling System}

The primary functions of the gas handling system are to store the helium when it is not in use in the primary system and.to remove radioactive contamination from the helium before returning it to the primary system or before discharging it from the plant. The basic components of the gas handling system are included on the Reactor System Flow Diagram, Dwg. 1963-B-5. All components which contain high pressure helium that may be contaminated with radioactivity are located within the containment vessel.

In order to store the $1680 \mathrm{lbs}$. of helium required to fill the primary loop, 40 storage cylinders are provided. Each cylinder is 18" OD $\times 16$ " ID $\times 20 \mathrm{ft}$. long and will store the helium at $3000 \mathrm{psi}$. The system is filled by first bleeding gas directly into the system until the equilibrium pressure of about $700 \mathrm{psi}$ has been reached. Then a transfer compressor is used to produce the desired pressure level in the primary loop. The transfer compressor is also used to transfer gas from the system back into the storage cylinders. The storage cylinders are arranged in 10 groups of 4 each. Each group has a separate connection to both the transfer compressor suction and discharge so that a portion of contaminated helium can be temporarily stored and then recirculated through cleanup equipment.

For the present design, we have selected two five-stage reciprocating compressors as offered by the Worthington Corp. Each unit is rated at $110 \mathrm{scfm}, 3000 / 14.7 \mathrm{psi}$. Since these compressors are designed for 
atmospheric pressure suction, a letdown valve is installed at the compressor inlet. This low suction pressure requirement also limits their capacity. For example, 13 hours are required to reduce the system pressure from its design point down to atmospheric pressure. For gas cycle reactor systems having gas volumes of the order of the 125 eMW-PBR or larger, a special compound compressor having a first stage consisting of a low specific speed centrifugal compressor followed by several reciprocating stages is desirable. This machine would be capable of taking suction directly at any system pressure and produce a maximum discharge pressure of 3000 psia by means of stage by-passing.

During normal operation, a portion of the helium is withdrawn from the system and filtered and cooled before passing through the charcoal traps. This gas then passes to the transfer compressor from whence it is stored in two high pressure storage cylinders. This clean high pressure gas is used as a source of sweep helium for the main compressor seals. Since this sweep helium ultimately bleeds back into the primary loop, the transfer compressor will run intermittently to maintain a pressure level of between 3000 and 1000 psia in these storage cylinders.

When it becomes necessary to dispose of the charge of helium, it will be bled to the stack via a separate cold trap that will reduce activity to acceptable levels. This cold trap and a vacuum system are located in the reactor service building. The primary function of the vacuum system is to initially out-gas the primary loop. Graphite parts for the core will have been given an outgasing treatment at the manufacturer's plant and will be shipped in sealed plastic bags in order to minimize the outgasing problem at startup.

\subsubsection{Emergency Procedures}

During a normal reactor shutdown, any one of the three main compressors can be left running to remove the decay heat. This heat will be transferred to the steam generator and steam will be vented to the condenser. However, there are three types of emergencies which could interrupt this normal procedure - loss of electrical power, failure of a compressor, and loss of primary system pressure.

When the system is at full pressure (i.e. when it has been running at full output), a single compressor can be used to remove the decay heat. 
The prewhirl vanes on this compressor can be adjusted to produce $15 \%$ of the total full load flow through the system which is more than adequate to remove decay heat. In the event of an interruption of the main source of electrical power to the compressors, a single compressor driven by the low speed direct coupled auxiliary motor will produce $10 \%$ of the total full load flow The auxiliary motor is connected to the emergency bus which is powered by both the small independent tie line and a dieselgenerator set.

In the event that a compressor is unable to operate, its discharge valve is shut to prevent backflow through that compressor. Since all three compressors are provided with an auxiliary motor, cooling would always be available since it is highly unlikely that more than two compressors would be out of service due to mechanical failure.

Although the steam generators are designed for forced water circulation at rated output, it is possible to remove the decay heat by natural water convection. Only the condensate return pump would bel connected to the emergency bus.

In the event of the loss of primary system pressure due to $z$ rupture, the minimum pressure in the system would be essentially the equilibrium pressure existing within the containment vessel. Frr the case where only helium is discharged into the containment vessel, this pressure is approximately $25 \mathrm{psig.} \mathrm{Under} \mathrm{this} \mathrm{condition,} \mathrm{coolant} \mathrm{flow} \mathrm{through} \mathrm{the}$ reactor would reach a minimum of $3 \%$ of the total full load flow. This flow would be produced by a single compressor driven by its main motor. It is considered highly unlikely that a loss of pressure incident would be combined with the loss of both main tie lines which would be the source of power for the main compressor motors.

Since the steam pressure is higher than the primary system helium pressure, a leak in a steam generator tube would result in moisture entering the primary system rather than helium entering the turbine generator system. The presence of moisture in any of the primary circuits will be first indicated by the moisture detector in that circuit. This signal will cause the reactor to scram and stop flow in that circuit by stopping the compressor and by shutting both sets of valves. The feedwater and main steam isolation valves for that steam generator, located just outside the containment vessel, are shut and steam side pressure is relieved through a control valve which insures that steam pressure remains above helium pressure at all times. 
Simultaneously with the relief of steam pressure, helium in the affected circuit would be transferred to certain of the storage tanks by the transfer compressor. The other two helium circuits would continue to operate for about 3 hours, by which time the system pressure would be lowered to essentially atmospheric pressure and subsequent decay heat removed by a single circuit. The charge of helium from the affected loop is then recycled through the cleanup equipment in the gas handling system, and maintenance can be undertaken on the steam generator.

The presence of water in the primary loop can cause an endothermic reaction with graphite. The threshold for this reaction is about $1300^{\circ} \mathrm{F}$ with significant reaction rates occuring above $1700^{\circ} \mathrm{F}$. Fixed graphite parts in the reactor will have been coated with one of the impervious materials, such as siliconized silicon carbide, which will prevent these parts from reacting with the water vapor. If sufficient water vapor gets into the reactor core to react with some of the fuel elements, the core loading can be replaced.

\subsubsection{Cleanup and Maintenance}

Perhaps the most significant penalty in permitting fission products to diffuse from the fuel elements is the effect on maintenance of equipment in the primary loop. Actually, this problem exists in a varying degree in practically all other reactor types. In the case of fixed fuel, liquid-cooled reactors, some radioactivity is induced in both the coolant itself and corrosion products dissolved in the coolant stream. In circulating fuel reactors, the entire fission product spectrum is circulated through the primary loop.

In the PBR, there is no induced activity in the coolant and no corrosion products so that the source of radioactivity will be those fission products which diffuse from the fuel elements and escape into the primary loop. Table 4.3-1 is a list of fission products which are volatile below $2500^{\circ} \mathrm{F}$ and therefore most likely to diffuse from the fuel elements.

Examination of this table indicates that the principal gaseous fission products fall into two general categories, namely those elements which can be solidified easily by compound formation and those which can be removed only by adsorption or refrigeration. Both of these techniques can be used to reduce fission product activity in the primary loop.

From the point of view of accessibility to equipment, it is 
TABLE $4.3,-1$

FISSION PRODUCTS VOLATILE AT OR BELOW $2500^{\circ} \mathrm{F}$

Total Activity

In Core

Nuclide $\quad$ BP, $\circ \quad \underline{\text { Half Life }}$ Gamma Energy, Mev (Mev/MW-sec) $10^{-13}$

Permanent Gases

$\mathrm{Kr}^{87}$

$-240$

$78 \mathrm{~m}$

$$
2.30
$$

1.89

50.5

0.41

41.5

09.0

$X e^{135}$

$-164$

$9.20 \mathrm{~h}$

0.52

29.6

0.25

47.5

Chemically Reactive Nuclides

$\begin{array}{lllll}\mathrm{Se}^{83} & 1300 & 25 \mathrm{~m} & \begin{array}{l}0.750 \\ 0.176\end{array} & \begin{array}{l}12.97 \\ 02.5\end{array} \\ \mathrm{Br}^{84} & 184 & 32 \mathrm{~m} & 0.89 & \begin{array}{l}47.8 \\ \end{array} \\ & & & 1.89 & 33.8 \\ \mathrm{Te}^{129} & 2500 & 74 \mathrm{~m} & 0.80 & 26.0 \\ & & & 0.30 & 09.76 \\ \mathrm{Te}^{131} & 2500 & 24.8 \mathrm{~m} & 0.7 & \\ & & & 0.16 & 29.7 \\ \mathrm{I}^{131} & 360 & 8.05 \mathrm{~d} & 0.64 & 09.28 \\ & & & 0.36 & 27.5 \\ & & & 0.28 & 01.74 \\ \mathrm{Te}^{132} & 2500 & 77.7 \mathrm{~h} & 0.231 & 33.0 \\ \mathrm{I}^{132} & 360 & 2.33 \mathrm{~h} & 0.60 & 34.5 \\ & & & 1.40 & 80.5 \\ & & & 2.00 & 06.2\end{array}$


FISSION PRODUCTS VOLATILE AT OR BELOW $2500^{\circ} \mathrm{F}$ (Cont'd)

Total Activity

In Core

Nuclide $\quad$ BP, $\bullet F$

Half Life Gamma Energy, Mev (Mev/MW-sec) $10^{-13}$

Chemically Reactive Nuclides (Cont'd)

$\mathrm{Te}^{133}$

2500

$2 m$

0.6

08.78

0.4

05.85

1.0

10.22

$I^{133}$

360

$20.8 \mathrm{~h}$

1.40

02.14

0.85

06.71

0.53

77.5

$\mathrm{I}^{134}$

360

$52.5 \mathrm{~m}$

1.78

15.5

1.10

71.5

0.86

47.8

$I^{135}$

360

$6.70 \mathrm{k}$

2.40

09.12

1.80

13.7

High Boiling Nuclides

$\mathbf{R b} \mathbf{B}^{88}$

1330

$17.8 \mathrm{~m}$

2.80

16.8

1.86

49.2

$5 r^{91}$

$\angle 100$

$9.67 \mathrm{~h}$

1.30

88.5

0.61

41.5

$\mathrm{Sb}^{126}$

2500

$28 d$

0.9

02.93

$\mathrm{Sb}^{127}$

2500

$93 \mathrm{~h}$

0.72

00.33

$\mathrm{Cs}^{138}$

1230

$32 \mathrm{~m}$

1. 44

271.4

0.980

79.4

0.46

28.8 
FISSION PRODUCTS VOLATILE AT OR BELOW $2500^{\circ} \mathrm{F}$ (Cont'd)

Total Activity

In Core

Nuclide BP, $F \quad$ Half Life Gamma Energy, Mev (Mev/MW-sec) $10^{-13}$

High Boiling Nuclides (Cont'd)

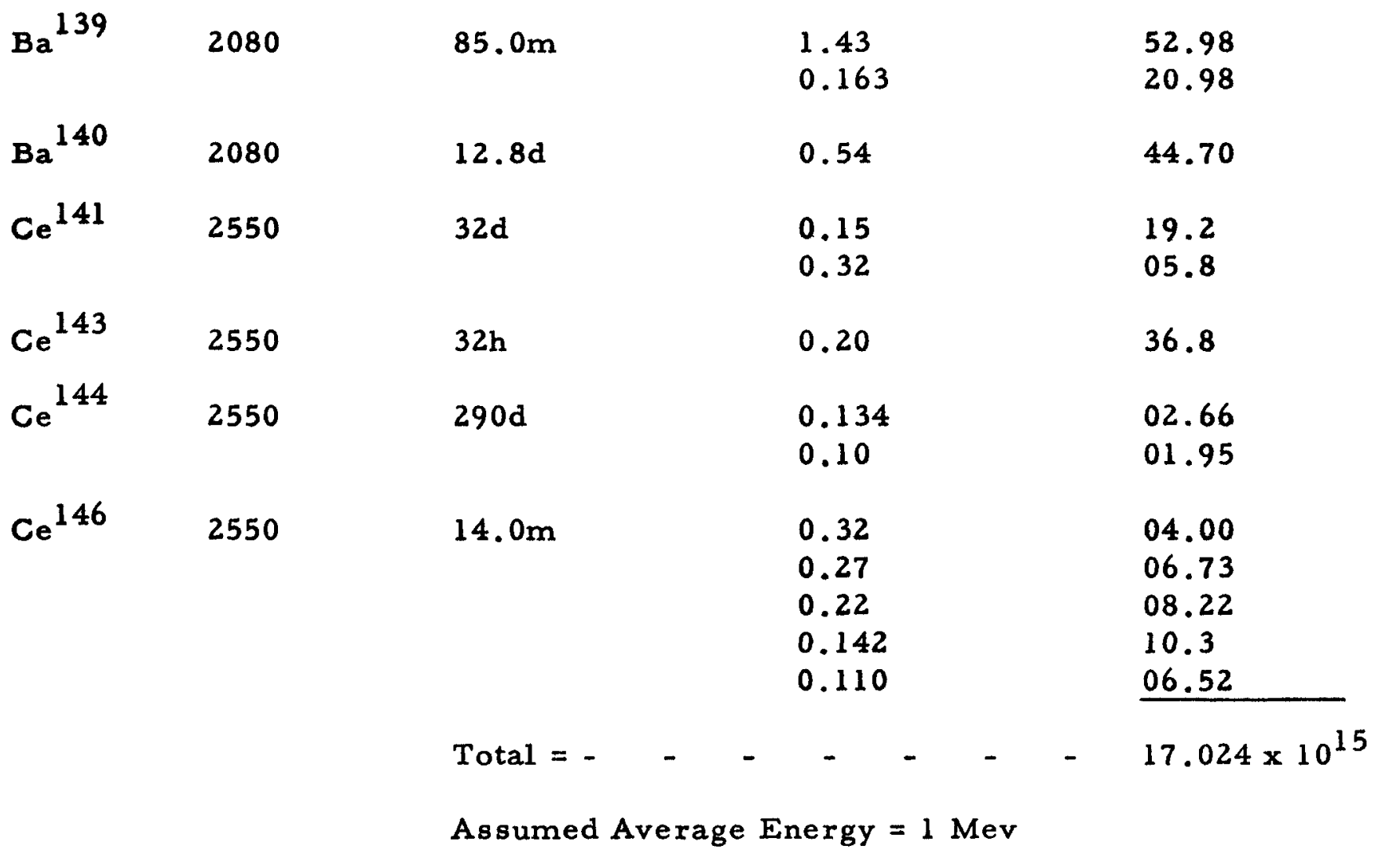


apparent that all short half-life species can be neglected since their natural decay would soon eliminate them. Decay chains which contain a long half-life species are of more concern. For example, Br-84 ( $1 \%$ yield) which decays to $\mathrm{Kr}-84$ need not be removed from the gas stream since its half-life is only 32 minutes and therefore about 3 hours after shutdown, activity due to this decay chain will cease to exist. $\mathrm{Br}-85$, on the other hand, although it has an even shorter half-life, gives rise to $10.6 \mathrm{y} \mathrm{Kr}-85$, which because of its much longer half-life, must be removed. In this case the bromine could be precipitated by reaction with silver or copper or the bromine can be ignored and the krypton removed by adsorption. Since $\mathrm{Br}-85$ has a half-life of only 3 minutes, the practical problems associated with precipitating it and removing the precipitate from the system before the $\mathrm{Br}-85$ decays to $\mathrm{Kr}-85$ (in which state it would revert to the gas phase) effectively rule out any consideration of bromine removal.

This situation is not nearly so clear-cut in the case of some chains containing iodine and tellurium. I-131, Te-132, and I-133 are all found in significant amount in fission yields and since their half-lives are 8 days, 3 days and 1 day respectively, it is not reasonable to wait for their decay. A chemical trap has therefore been provided in the hot end of each of the steam generators as shown in Section 4.3.1. This trap will contain refractory spheres carrying a precipitant such as copper or silver. The trap is so constructed as to be able to discharge some or all of the spheres and replace them with fresh ones. The exact nature of this discharge schedule is not known at this time due to the unusual nature of the process, i.e. chemical reaction between an anion and a cation with the cation disappearing due to radioactive decay.

This chemical trap will of course remove all of a given chemical element from the gas stream and not just those isotopes which give rise to long-lived activity. Thus $I-136$ will be removed as readily as $I-137$, though we are not concerned over activity due to I- 136 which will decay to essentially nothing in 10 minutes and are concerned over I- 137 which is a precursor of $30 \mathrm{y} \mathrm{Cs}-137$.

Decontamination of the equipment involves removal of any radioactive dust which may have settled out on equipment surfaces during reactor operation. For example, all the kryptons decay through rubidium to strontium, both of which will probably settle out on various surfaces of the primary circuit. It is improbable that any form of chemical bond between the various fission product daughters and the primary equipment surfaces will exist and any particulate matter will be held by 
weak Van der Waal forces. For this reason acid etching, which has been resorted to in those cases where the fission products are precipitated from a solution onto the equipment wall, should not be required in this system. It is our belief that essentially all of the deposited activity will be removable by flushing with water or a mild detergent solution.

Consequently, the primary system has been designed using the direct maintenance philosophy rather than one involving remote cutting, manipulating and rewelding of the components. In the case of the steam generators discussed in Section 4.3.1, leaking tubes can be plugged from behind a shielding wall, thus requiring no decontamination of the steam generators. Maintenance on the compressors will be performed after they have been decontaminated with rinse solutions. The compressors will be fitted with a number of spray heads. Rinse solutions will be prepared in the reactor service building and piped directly to the spray heads. The waste solution is then drained and pumped to an aqueous waste disposal facility on the site. The dual block valves in the primary circuit prevent rinse solution from entering the reactor. Withdrawal space is provided for the compressor wheel assembly. The seal on the anti-drive end of the blower is accessible by removing a cover over the shaft end and the seal on the drive end is accessible by removing a stub shaft between the drive motor and compressor. After maintenance has been completed, residual moisture from the rinse solution will be removed either by recirculating dry helium through the gas handling system or utilizing the vacuum equipment. 


\subsection{Shielding and Containment}

\subsubsection{Shielding Design}

Shielding is provided in this design for three distinct but correlated conditions. These conditions are:

a) Shutdown shielding for maintenance and refueling.

b) Biological shielding during full power reactor operation.

c) Shielding for a maximum system rupture to permit orderly and safe evacuation of the plant site.

The shield design which most adequately meets the design conditions consists of a primary or core shield and a secondary or containment vessel shield. The primary shield serves as a biological shield for the core during shutdown for refueling and maintenance. The primary shield also strongly attenuates radiation from the core during full power operation but not to the extent of permitting access to the vapor container during power production.

The secondary shield attenuates the remaining core radiation plus radiations from the primary loop to a level below established tolerance levels at the surface of the secondary shield during full power operation. The secondary shield also attenuates radiation from a maximum system rupture and a postulated core disintegration to a level which will permit orderly and safe evacuation of the plant site without exceeding maximum emergency dose tolerances.

The primary shield design which evolved from the shielding analysis consists of a primary shield of ordinary concrete 4 feet thick and a secondary shield of ordinary concrete $2-1 / 2$ feet thick. The primary shield has a water tank 2 feet thick to thermalize fast neutrons from the core.

The shutdown dose rate with the reference shield design will be initially about $20 \mathrm{mr} / \mathrm{hr}$ and will decay to approximately $10 \mathrm{mr} / \mathrm{hr}$ in 8 hours. This is based on access to the vapor container 2 hours after shutdown. Thus, for an 8 hour maintenance or refueling exposure, the maximum accumulated dose would be approximately $120 \mathrm{mr}$. The permissible dose based on continuous exposure is $300 \mathrm{mr} /$ week which would permit 20 hours per week access per individual. 
The secondary shield thickness was predicated on maximum system rupture since this source of radiation is many magnitudes greater than the radiations to be attenuated by the secondary shield during full power operation. It was conservatively assumed that shielding adequate for emergency evacuation would be more than adequate to reduce full power radiation to below $6 \mathrm{mr} / \mathrm{hr}$ at the surface of the secondary shield.

The plant control room was assumed to be the only plant area which could not be immediately evacuated at the time of major system rupture. It was further assumed that the operator who must secure the plant before evacuation could be exposed to a maximum dose of $25 \mathrm{R}$ which is an accepted. one shot dose in case of emergency. The $2-1 / 2$ feet of secondary shielding based on the conservative analysis of the incident presented in Part II, Section 1.0 would allow for 50 minutes to secure the plant and to evacuate the site.

\subsubsection{Containment Vessel}

The containment vessel is shown on Drawing 1963-C-7. It serves the dual function of a housing for the primary system equipment and an ultimate barrier to prevent the escape of radioactivity to the surrounding area in the event of a major reactor incident. The cylindrical containment vessel is $75 \mathrm{ft}$. diameter and has an overall height of $94 \mathrm{ft}$.

The containment vessel wall thickness has been set on the basis of an incident which might involve the simultaneous release of all the helium in the primary loop and all of the water from only one steam generator, since the three generators are not interconnected within the containment vessel. The resultant pressure rise for an adiabatic expansion of the helium water into the containment vessel is 31.6 psig. at an equilibrium temperature of $210^{\circ} \mathrm{F}$. On this basis, a $1^{\prime \prime}$ vessel wall will be adequate. Further study of the rate of heat exchange to the concrete mass and heat loss through the vessel wall would undoubtedly reduce the required wall thickness.

The bottom of the vessel is 41 feet below grade and is supported on a continuous concrete pad beneath the vessel. Because of its size, the vessel will have to be field erected. After pouring the concrete support pad, the vessel would be fabricated on supports above the pad. This permits the vessel to be completely leak tested, X-rayed, stress relieved and structuraly tested. The vessel will then be lavered onto the pad, and grouted in. A large access opening will be cut in the containment vessel above grade to permit installation of the equipment after which the opening will be rewelded and the vessel reinspected. 


\subsection{Secondary System}

Since one of the advantages of the PBR concept is its capability of operating at high reactor outlet temperatures with consequent high plant thermal efficiencies, one of the primary considerations in the design of the steam cycle was to take advantage of the economies granted by this characteristic. Maximum obtainable cycle efficiencies can be achieved by using the most advanced designs such as supercritical pressures, multiple stages of reheat, etc. Multiple stages of reheat would introduce complexities of piping, and increase the number of penetrations of the containment vessel, while the relatively untried and experimental techniques of supercritical pressure are felt to be unwarranted at this stage of development of the PBR. Therefore, it was decided to use a conventional steam cycle, employing a simple arrangement for the first design of a nuclear power plant of this type.

The power plant design evolved in accordance with the above reasoning, and a non-reheat steam cycle was selected with steam conditions of $1450 \mathrm{psig} / 1000^{\circ} \mathrm{F}$, a turbine exhaust pressure of $1.5^{\prime \prime} \mathrm{Hg}$. abs, and four stages of regenerative feedwater heaters producing a final feedwater temp. of $400^{\circ} \mathrm{F}$. A flow diagram of the primary steam and water systems is shown on Drawing No. 1963-C-17. A preliminary plant heat balance and general specifications for the major steam cycle equipment is included in the following sections:

\subsubsection{Heat Balance}

The complete heat balance for the maximum capability of the Pebble Bed Reactor Steam Power Plant is shown on Drawing No. 1963-C-16. The following is a tabulation of significant parameters:

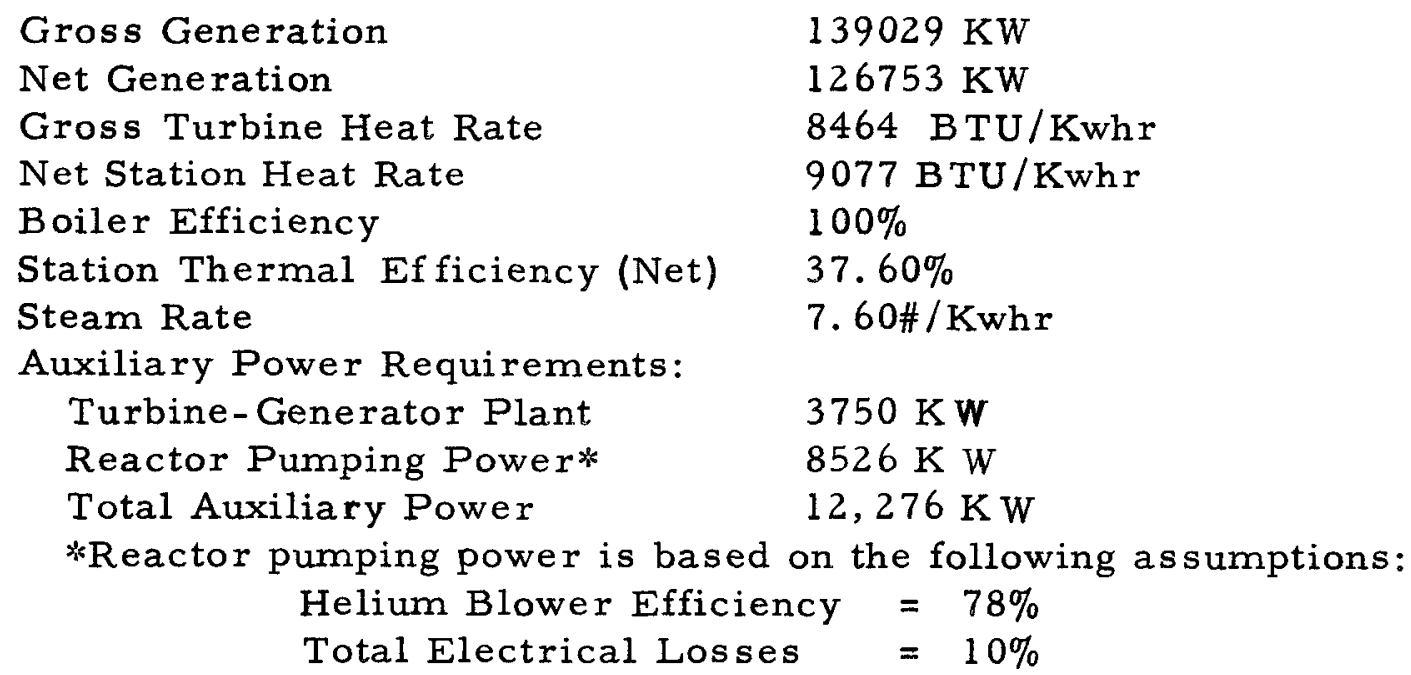




\subsubsection{Turbine Generator}

A. Turbine

Rated Capacity

Gross Capability

Type and Speed

Throttle Pressure

Throttle Temperature

Number of Extraction Points

Design Back Pressure
$125,000 \mathrm{~K} \mathrm{~W}$.

$139,029 \mathrm{~K} \mathrm{~W}$

Tandem compound, triple flow

exhaust, condensing, non-reheat, $3600 \mathrm{rpm}$

1450 psig

$1000^{\circ} \mathrm{F}$

4

1. 5" $\mathrm{Hg} \mathrm{Abs}$

1. 5" $\mathrm{Hg} \mathrm{Abs}$

B. Generator

Rating

Power Factor

$160,000 \mathrm{kva}$

Cooling: Hydrogen

0.85

Excitation
(a) Main Exciter
- Shaft Driven

(b) Spare Exciters

- Motor Driven

\subsubsection{Auxiliary Eguipment}

A. Condenser

Surface Area

Steam Condensed

Number of Passes

Back Pressure

Cooling Water Flow

Cooling Water Design Temp.

Cooling Water Tube Velocity

Tube Cleanliness Factor

Number of Tubes

Tube Size and Gage

Overall Tube Length

Tube Material

Tube Sheet Material

Method of Securing Tubes to

Tube Sheets

Special Features:

Provision for continuous dump of $1,055,000 \mathrm{H} / \mathrm{hr}$ main steam (desuperheated and reduced in pressure).
$80,000 \mathrm{Ft}^{2}$

771,540 \#/hr

2

1. 5" $\mathrm{Hg}$ Abs

$64,014 \mathrm{gpm}$

$60^{\circ} \mathrm{F}$

$6.5 \mathrm{Ft} / \mathrm{Sec}$.

$85 \%$

10,246

$1 " \mathrm{OD}, 18 \mathrm{BWG}$

$30 \mathrm{Ft}$.

Admiralty

Silicon Bronze

Welded 
B. Feedwater Pumps (Two Required)

Type

Capacity

$\mathrm{TDH}$

Efficiency

Motor Drive
9-Stage Horizontal

$1225 \mathrm{gpm}$

$4140 \mathrm{Ft}$.

$77 \%$

$1750 \mathrm{HP}, 3600 \mathrm{rpm}$

C. Closed Feedwater Heaters (Three Required)

Type

Shell Design Pressure

and Temperature:

Heater No. 4

Heater No. 3

Heater No. 1

Tube Design Pressure

and Temperature:

Heater No. 4

Heater No. 3

Heater No. 1

D. Deaerating Heater

Type

Storage Tank Capacity

Design Pressure

Capacity
Horizontal

300 psig, $650^{\circ} \mathrm{F}$

150 psig, $475^{\circ} \mathrm{F}$

Vacuum to $100 \mathrm{psig}, 450^{\circ} \mathrm{F}$

E. Condensate Water Pumps (Two Required)

Type

Vertical

Capacity

$\mathrm{TDH}$

$1840 \mathrm{gpm}$

Motor Drive

310 Feet

$100 \mathrm{HP}, 1800 \mathrm{rpm}$

Tray with Internal Vent Condenser

12 Minutes

50 psig

$1,055,275 \# / \mathrm{hr}$

F. Circulating Water Pumps (Two Required)

Type

Capacity

$\mathrm{TDH}$

Motor Drive

G. Principal Piping

Service

Material
Vertical

$33,250 \mathrm{gpm}$

$28.4 \mathrm{Ft}$.

$350 \mathrm{HP}, 710 \mathrm{rpm}$

Main Stearn

Seamless $1-1 / 4 \% \mathrm{Cr}-1 / 2 \%$ Mo ASTM Spec., A-335, Grade P11 


\section{H. Water Treatment Systems}

(1) Filtered Water System

One (1) Reactivator

Two (2) Pressure Filters

Two (2) Activated carbon filters

Storage tank capacity - 250,000 Gal.

Maximum continuous capacity - $300 \mathrm{gpm}$

(2) Make-Up Water Demineralizing System

One (1) Cation Demineralizer

One (1) Anion Demine ralizer

One (1) Mixed Bed Demineralizer

Capacity - $20 \mathrm{gpm}$

Maximum Total Solids in Effluent - $50 \mathrm{ppb}$

Storage Capacity - $4250 \mathrm{Gal}$.

(3) Condensate Water Scavenging Demineralizer

Two (2) Cellulose Filters

Two (2) Mixed Bed Demineralizers

Capacity - 832 gpm (50\% of Condensate Flow)

Maximum Total Solids in Effluent - $50 \mathrm{ppb}$

\subsubsection{Electrical Equipment}

The electrical requirements for the $125 \mathrm{eMW-PBR}$ are shown on a single line diagram, Drawing 1963-B-15. The $160 \mathrm{MVA}, 15 \mathrm{KV}$ generator is provided with such instrumentation, relays, voltage regulating equipment, and protective devices as is customarily furnished with turbinegenerators in conventional steam power houses. A shaft driven exciter and a spare exciter driven by a 4000 volt motor connected to the station auxiliary system are also provided.

A 140 MVA transformer transforms the generator voltage into the high voltage of the utility system, which was assumed to be $115 \mathrm{Kv}$. Since the electrical distribution system is difficult to postulate unless a particular site location and existing generating facilities are defined, it was arbitrarily decided to exclude from this study, all electrical equipment beyond the high voltage terminals of the main transformer. Therefore, the estimated cost of the electrical equipment does not include the outdoor high voltage switchyard or any other portion of the electrical distribution system. 
For power supply to the station auxiliaries and controls the following voltage sources are available:

(a) Two 4160 volt bus systems for large motors.

(b) Two 480 volt bus systems for motors of $150 \mathrm{hp}$.

(c) Two 125 volt d-c systems supplied by batteries. for controls and emergency standby motors.

The 4160 volt system is normally supplied by a 15,000 Kva auxiliary transformer, connected on the primary side to generator voltage. A reserve transformer of the same size takes the power from the high voltage svstem. The following major motors are connected directly to the 4160 volt system:

(a) Three (3) motor drives for the helium blowers.

(b) Two (2) motor drives for steam generator feed water pumps.

(c) Two (2) motor drives for circulating water pumps.

(d) One (1) motor drive for the spare exciter.

The 4160 volt system also supplies power to the 480 volt auxiliary buses through two (2) $1000 \mathrm{Kva}$ transformers. Either of the two transformers has sufficient capacity to provide for the complete 480 volt system. The major drives connected to this system are for the condensate pumps, the service water pumps, the fire water pumps, the turbine auxiliary oil pump, the containment vessel space cooling equipment, and the reactor service building ventilation equipment.

In the event of an automatic trip of the turbine generator the entire auxiliary system would be automatically transferred to the reserve transformer as is usual in conventional power stations. Additional emergency power is provided for functions which are essential in any emergency. Such functions are the scram shut down of the reactor and emergency minimum helium and feedwater flow. It is proposed to have two (2) batteries independent of each other, each with an independent charger. All particularly sensitive d-c fed equipment would be connected to the batteries through automatic three-way switches. The emergency helium blower motors and emergency boiler fill pump motors are connected to a separate 480 volt emergency bus, consisting of two sections. One emergency boiler feed pump and at least one emergency helium blower is connected to each of these sections. The emergency system can be fed from any of the 1000 Kva transformers by tying through breakers to the 480 volt auxiliary buses, or from any of two separate emergency power sources. One of these is assumed to be a tie to a nearby energy source, the other an auxiliary diesel generator. 
If the turbine generator and the auxiliary transformer trip, the main motor of one of the helium blowers will be automatically disconnected from the 4160 volt system, and the corresponding emergency motor connected to the 480 volt emergency bus after the speed of the blower is reduced below $450 \mathrm{rpm}$. At the same time an emergency boiler fill pump is automatically connected to the same bus. Disconnection of these motors may be done manually if it is verified that they are not needed. If the 480 volt bus system loses voltage, the emergency bus sections are automatically transferred to the emergency power sources.

In order to keep the isolated phase generator leads as short as possible, the main transformer is located on an outdoor transformer platform at grade level immediately aft of the generator. The auxiliary and reserve transformers are located on this transformer platform on either side of the main transformer. The electrical switchyard is located across the service road from the transformer platform. 


\section{6 Control and Instrumentation}

The functions of the Pebble Bed Reactor control system are to provide a safe method of start-up and shut-down and enable the reactor system to follow the load demands placed upon the turbine generator system. Since a unit of this size would probably supply a part of the utility's base load, load swings are likely to occur at a moderate rate. The control system should act to maintain constant turbine inlet temperature over the major portion of the operating range in order to insure maximum economy. It is also desirable to maintain constant reactor inlet and outlet temperatures in order to avoid transient temperature stress problems.

A detailed evaluation of the heat capacities, transient times, and component performance characteristics together with the methods and devices of meeting the control objectives has not been performed. However, the basic outlines of the control system have been established.

Considering a decrease in generator load, this will cause the main turbine throttle to start closing and a corresponding decrease in steam flow. Since less heat is being removed from the helium passing through the steam generator, both the reactor inlet and outlet temperatures will tend to rise. These temperature signals can be used to decrease power by inserting the control rods and/or by the effect of the negative temperature coefficient of reactivity in the core. At the same time, the helium flow will be decreased, thus tending to maintain constant reactor tem.-. peratures. At reduced loads the steam outlet temperature from the steam generator would tend to rise because the terminal difference at the superheater outlet will decrease as the load decreases. If this $t$ erminal difference is kept small, as it will be for metallurgical reasons, the increase in steam temperature can be kept small. If necessary, it can be completely controlled by attemperation.

In essence, this system would maintain constant reactor temperature by controlling reactor power and helium flow. Variation in helium mass flow can be accomplished by varying the system pressure and by adjusting the prewhirl vanes at the compressor inlet. System pressure variation will require the use of the transfer compressor over most of the range. This limits the rate of pressure change (i.e. rate of flow change)and consequently this method would be used for slow power changes to a new steady operating level. The adjustable prewhirl vanes affect the axial component of flow into the main compressors and can be used 
to accommodate rapid power changes over a range of about $2: 1$.

As shown in Section 1.4.8 of Part II, the temperature coefficient of reactivity for the reference design is $-2.7 \times 10^{-5} /{ }^{\circ} \mathrm{F}$. This value is an order of magnitude below that of a typical pressurized water reactor. However, it could prove sufficient to accommodate the normal power changes that are typical of a base loaded central station unit as opposed to the requirement of an isolated power unit or a propulsion power plant.

\subsubsection{Reactor System Instrumentation}

For estimating purposes, a typical reactor control circuit was considered for the PBR. This included two start-up channels, a log power and period channel, three safety channels and a control channel. Neutron flux indicators will be located in the water shield directly outside the reactor pressure vessel. At this point, the neutron flux is less that a factor of $10^{3}$ below the peak flux in the core.

Process instrumentation will be installed in the primary loop to provide signals for flow, pressure, and temperature. Flow tubes will be installed in each of the three primary circuits. Pressure transmitters will be hermetically sealed. In order to detect possible steam leakage into the primary loop, a small helium stream will be bled from each compressor discharge through a moisture detector and returned to compressor suction. Moisture detectors which can detect the presence of 1 ppm water vapor in an inert gas stream are available.

\subsubsection{Steam Plant Instrumentation}

In the turbine generator system, the type of feedwater control used will depend on whether the steam generator is a once-through or a controlled circulation design. For estimating purposes it was assumed that a conventional three element feedwater control system was used similar to that employed on most conventional steam power plants. In order to protect the feedwater pumps against overheating at low loads, an automatic recirculation control system is provided for each pump.

The condensate control system is similar to that used on most conventional steam systems. The condenser hotwell level is controlled by throttling the condensate pump discharge. The level in the deaerator storage tank is controlled by either diverting surplus condensate to the 
condensat $\epsilon$ storage tank on rising level caused by falling load, or permitting the condensate in the condensate storage tank to flow into the condenser hotwell and consequently to the deaerator storage tank on falling deaerator level caused by rising load.

Condenser hotwell, deaerator, condensate and other storage tanks, and feedwater heaters will be equipped with float switches to actuate annuciator alarms in the event of high or low levels. 
Section 5.0

Cost Analysis

Page

5.1 Capital Costs $\ldots \ldots \ldots \ldots \ldots \ldots \ldots \ldots \ldots \ldots \ldots \ldots \ldots \ldots$ 5-1

5.2 Fuel Costs $\ldots \ldots \ldots \ldots \ldots \ldots \ldots \ldots \ldots \ldots \ldots \ldots \ldots \ldots$ 5-1

5.3 Operating Costs $\ldots \ldots \ldots \ldots \ldots \ldots \ldots \ldots \ldots \ldots \ldots \ldots \ldots . \ldots . \ldots$

5.4 Production Costs $\ldots \ldots \ldots \ldots \ldots \ldots \ldots \ldots \ldots \ldots \ldots \ldots \ldots$ 5-3 


\subsection{Cost Analysis}

\subsection{Capital Costs}

Capital costs of the 125 eMW-PBR are summarized in Table 5.1-1. Total direct costs amount to $\$ 19,240,000$. Indirect charges, including $5 \%$ for omissions and contingencies on the conventional steam plant and $30 \%$ for omissions and contingencies on the reactor portion of the plant, amount to $\$ 12,981,000$, or $67.5 \%$ of the direct costs. This results in a total estimated cost of $\$ 32,221,000$ or $\$ 254.20$ per net kilowatt output, based on a maximum net capability of $126,753 \mathrm{KW}$.

The breakdown on total costs between the reactor and power generating portions of the plant are as follows:

Reactor Plant

Turbine generating plant
$\$ 15,049,000$

$\$ 17,172,000$
$\$ / \mathrm{KW}$

118.70

135.50

A summary of the accounts of the construction cost estimate is given in Section 5 of Part II of this report.

These costs are based on the assumption that the necessary research and development has been completed, including operation of a reasonable size prototype through several fuel replacement cycles. Engineering design of all equipment is included in its selling price, in accordance with current practice for power plant equipment. In arriving at this estimate we have incorporated what we believe to be reasonable safety features. Additional requirements will obviously increase the estimate.

\subsection{Fuel Costs}

The 125 eMW-PBR is a typical nuclear power plant with respect to the uncertainty of fuel costs. A deliberate effort has been made to err on the conservative side which is typified by selecting the most pessimistic conversion ratio upon which to base fuel costs.

The design conversion ratio is 0.863 , based on batch-loading of the core, a $K_{\text {eff }}$ of 1.10 and a values of $U-233$ as developed by $R$. L. Murray of North Carolina State for Alco Products. More optimistic fuel costs result from a continuous loading schedule and the use of ORNL a values of $\mathrm{U}-233$. 
TABLE $5.1-1$

\section{Capital Cost Summary}
A - Land \& Land Rights
$\$ 350,000$
B - Structures \& Improvements
$2,819,000$
C - Reactor Plant
$8,361,000$
D - Turbo-generator Plant
$6,003,000$
E - Accessory Electrical Equipment
854,000
F - Transmission Plant
566,000
G - Misc. Power Plant Equipment
287,000

Sub-total

$\$ 19,240,000$

H - Omissions \& Contingencies

$2,955,000$

I - Construction Plant \& Job Adminstration

$3,329,000$

$J$ - Engineering \& Construction Fees

$2,042,000$

K - Clients Costs

$4,655,000$

Total

$\$ 32,221,000$ 
The design fuel cycle is 100 full power days or 125 days at $80 \%$ load factor. Blanket life is 1000 full power days or 1250 days at $80 \%$ load factor. One tenth of the blanket is unloaded with every core loading.

Fuel costs are based on a reactor rating of $350 \mathrm{MW}$. The heat generated in a core lifetime is equal to:

$$
350000 \times 3415 \times 24 \times 100=2.87 \times 10^{12} \mathrm{BTU} .
$$

Fuel burnup rate is $0.48 \mathrm{Kg}$ per $\mathrm{MW}$ year. Total atom burnup is $0.021 \%$ in the core and $0.101 \%$ in the blanket.

Table 5.2-1 presents a fuel cost summary per core loading based on equilibrium conditions, i.e. after 10 cores have been used. Upper and lower limits result from maximum and minimum fuel fabrication and shipping costs. Figure 5.2-1 present these data in a bar graph. This range, of 15.1 to 21.9 cents per million BTU brackets cost of fuel in the Ohio River Basin, one of the lowest fuel cost areas in the United States.

\subsection{Operating Costs}

No attempt has been made to detail operating costs which include wages, (including supervision), water, lubricating.oil, interim replacements and general maintenance. A figure of one mill per kilowatt hour has been used in the production cost estimates.

\subsection{Production Costs}

Production costs are composed of the fixed charges on the capital investment, plus operating and fuel costs. Since the locale of this plant or the method of financing has not been established, fixed charges of $14 \%$ have been assumed. This covers equity before taxes, insurance, taxes and amortization of the plant.

Production costs have been based on an $80 \%$ load factor or 7000 full power hours per year and are summarized in Table 5,4-1 for minimum and maximum fuel costs. 
TABLE 5.2-1

\section{Fuel Cost Summary Per Core Loading Equilibrium Conditions, \\ Blanket/Core Lifetime $=10$}

\begin{tabular}{|c|c|c|c|}
\hline \multicolumn{2}{|c|}{ Minimum } & \multicolumn{2}{|c|}{ Maximum } \\
\hline $\begin{array}{l}\text { otal } \overline{\text { Cost }} \\
\$ / 10^{3}\end{array}$ & $\begin{array}{l}\text { Heat Cost } \\
\phi / 10^{6} \mathrm{BTU}\end{array}$ & $\begin{array}{c}\text { Total Cost } \\
\$ / 10^{3}\end{array}$ & $\begin{array}{l}\text { Heat Cost } \\
d / 10^{6} \text { BTU }\end{array}$ \\
\hline
\end{tabular}

1) Core Fabrication

84.6

2.95

174.0

6.06

2) Blanket Fabrication

39.0

1.36

130.0

4.53

3) Inventory

96.5

3.36

96.5

3.36

4) Burnup \& Losses

108.0

3.76

108.0

3.76

5) Shipping

28.7

1.00

43.0

1.50

6) Reprocessing

78.0

2.72

78.0

2.72

Totals

434.8

15.15

629.5

21.93 
FIGURE 5.2-1

FUEL COSTS SUMMARY

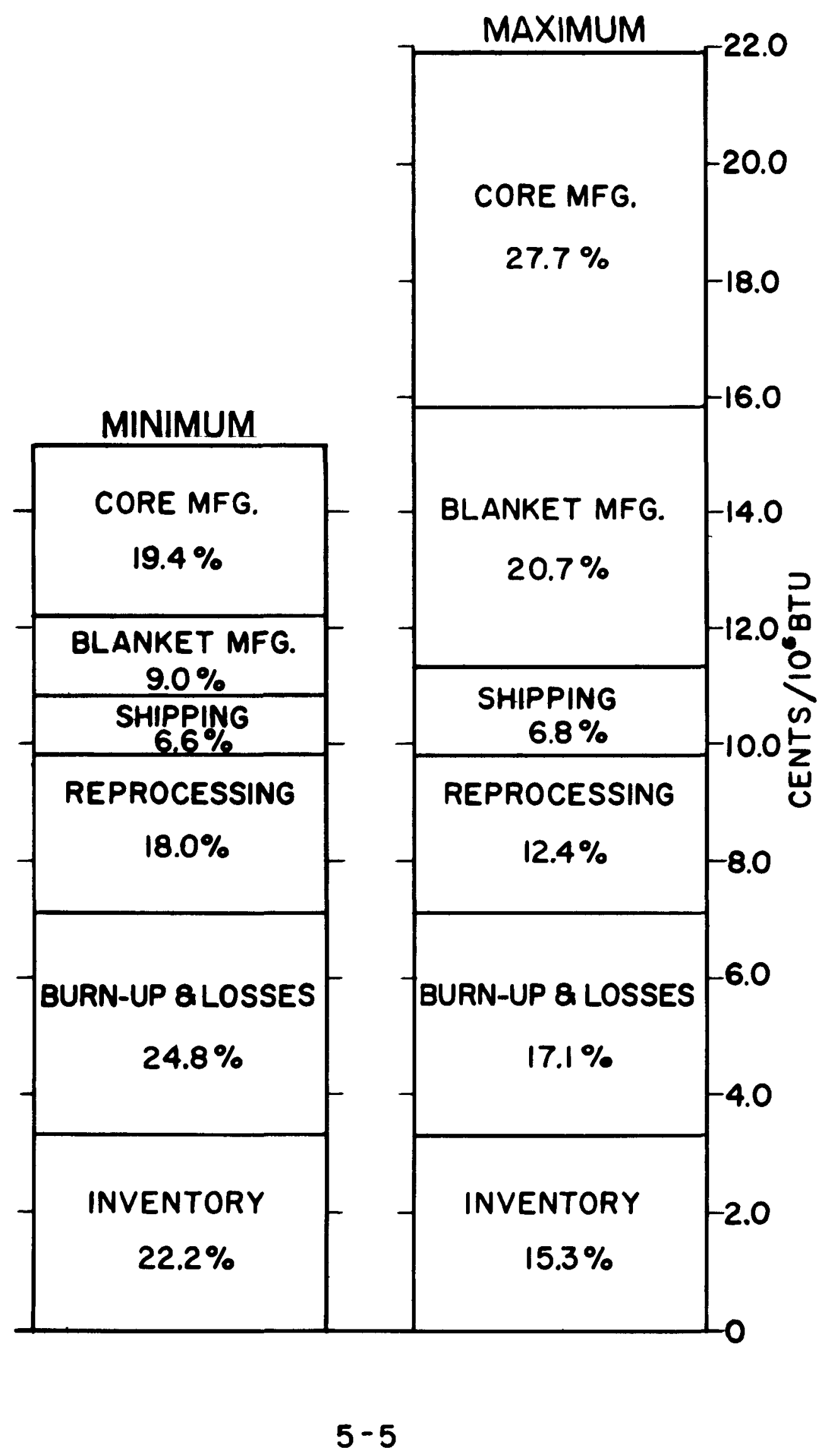


TABLE $5.4-1$

125 eMW-PBR Production Costs

Investment Data ( $\$ / \mathrm{KW})$

Property \& Improvements

$$
7.05
$$

Turbine Plant Structures

11.35

Turbine Plant

97.80

Reactor Plant Structures

24.40

Reactor Plant

94.30

Electrical \& Miscellaneous Total

$\frac{19.30}{254.20}$

Fuel Data $\left(\notin / 10^{6} \mathrm{BTU}\right)$

Fabrication

Inventory \& Burnup

Shipping

Reprocessing

Total

Min.

4.31

7.12

1.00

$\frac{2.72}{15.15}$
Max.

$\overline{10.59}$

7.12

1.50

$\frac{2.72}{21.93}$

Cost of Energy (mills/KWh)

Plant at $14 \%$ fixed charges

\& $80 \%$ load factor

Operation

Fuel

Total
5.08

1.00

1.38

7.46
5.08

1.00

1.99

8.07 
Section 6.0

Drawings for Part I

Reference has been made to the following drawings throughout the text of Part I.
$1963-B-5$
Reactor System Flow Diagram
$1963-C-7$
Reactor System Sectional Elevations
$1963-B-8$
Reactor System Sectional Plan
$1963-B-10$
Plat Plan
$1963-B-11$
Plan of Turbine Generator Building and Containment Vessel
$1963-B-12$
Plan of Turbine Generator Building and Containment Vessel
$1963-B-13$
Plan of Turbine Generator Building
$1963-B-14$
Elevation of Containment Vessel and Section "A-A"
$1963-B-15$
Electrical One Line Diagram
$1963-C-16$
Heat Balance
$1963-\mathrm{C}-17$
Flow Diagram of Primary Steam and Water Systems
$1963-B-19$
Reactor Assembly
$1963-B-20$
Steam Generator
AEL-271
Control Rod Drive
B \& W $66261 \mathrm{E}$
Preliminary Design of Helium Heated Steam Generator $\mathrm{J}-1194$ Assembly of Barrel Compressor (De Laval) 


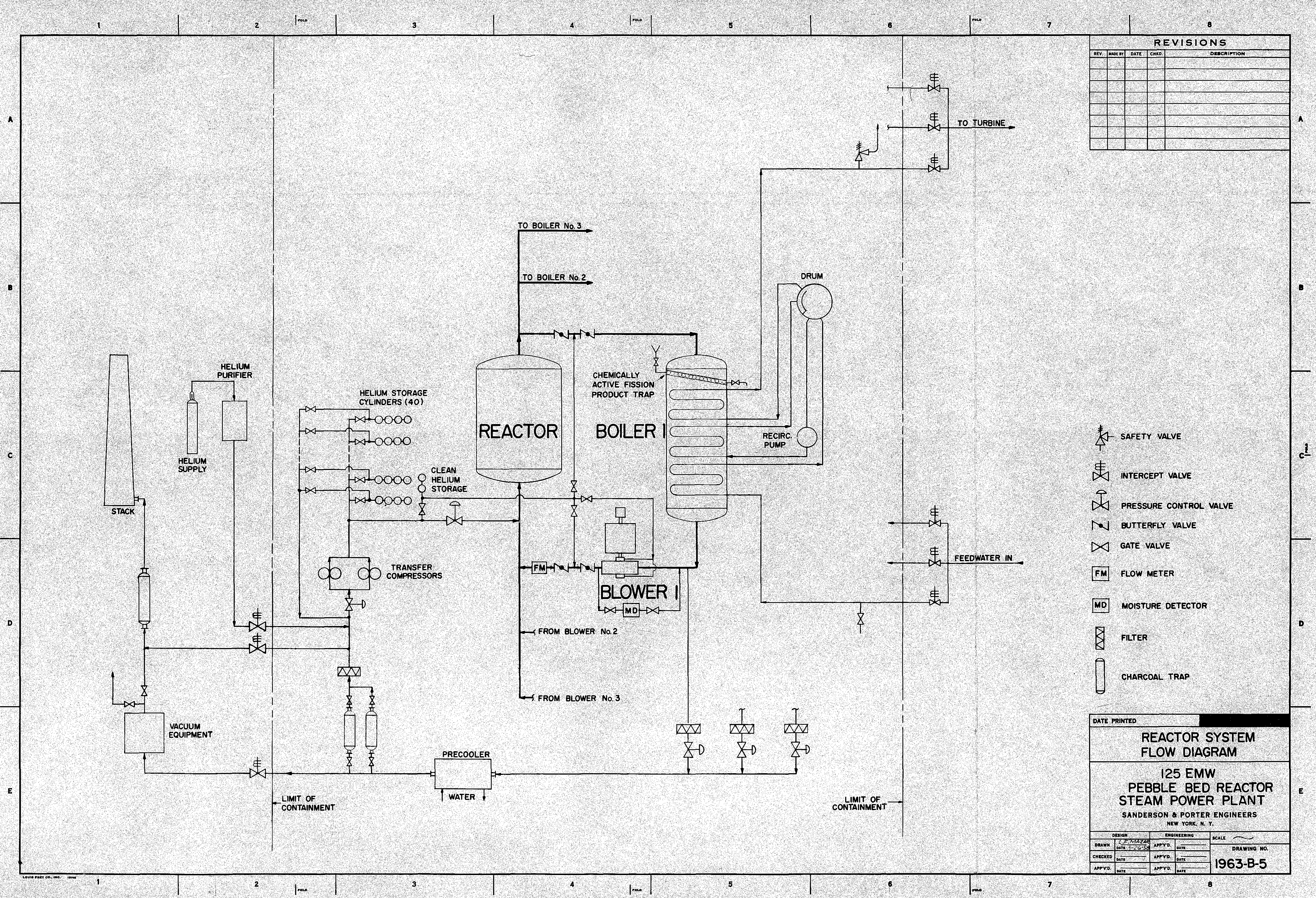




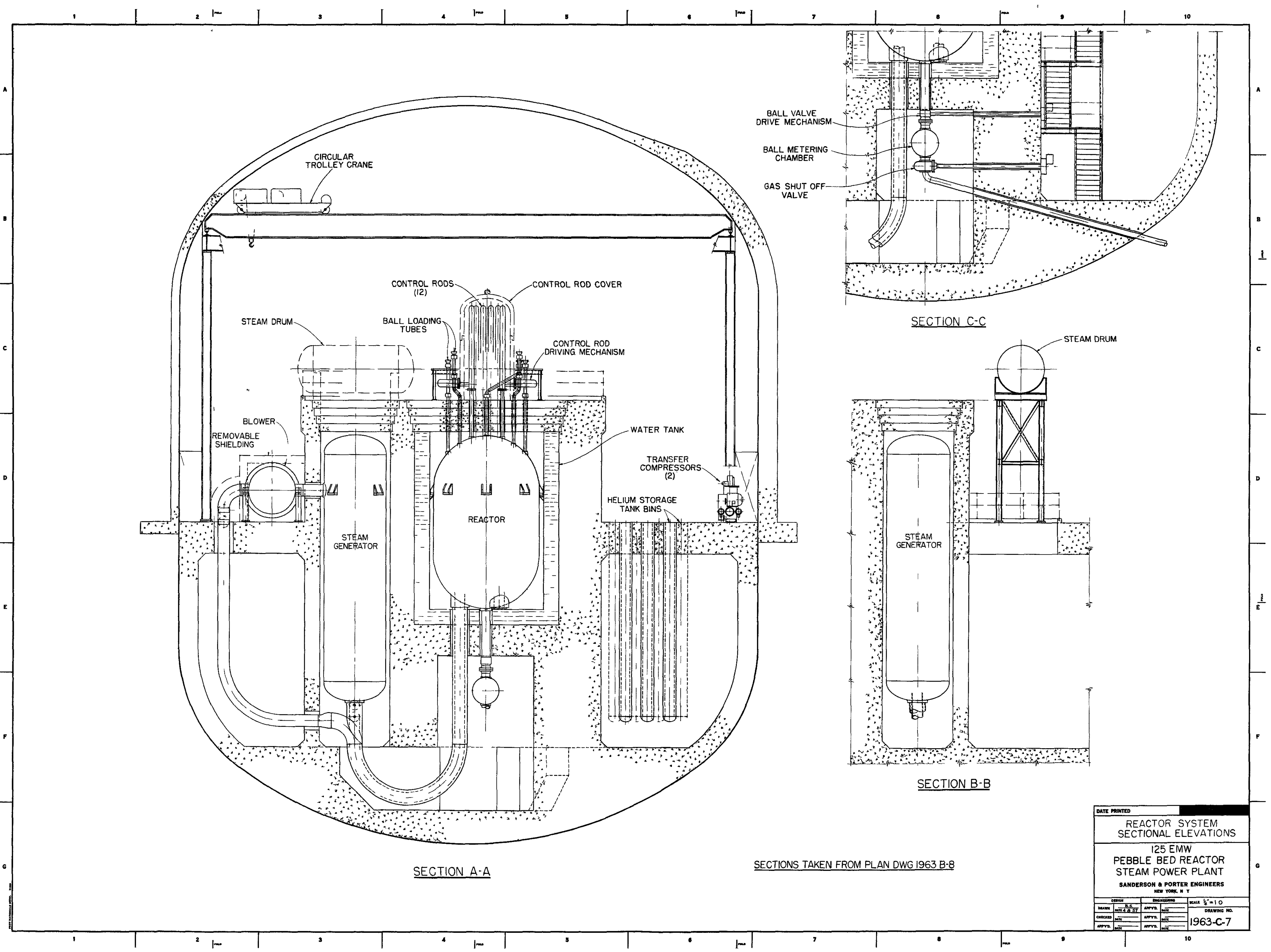




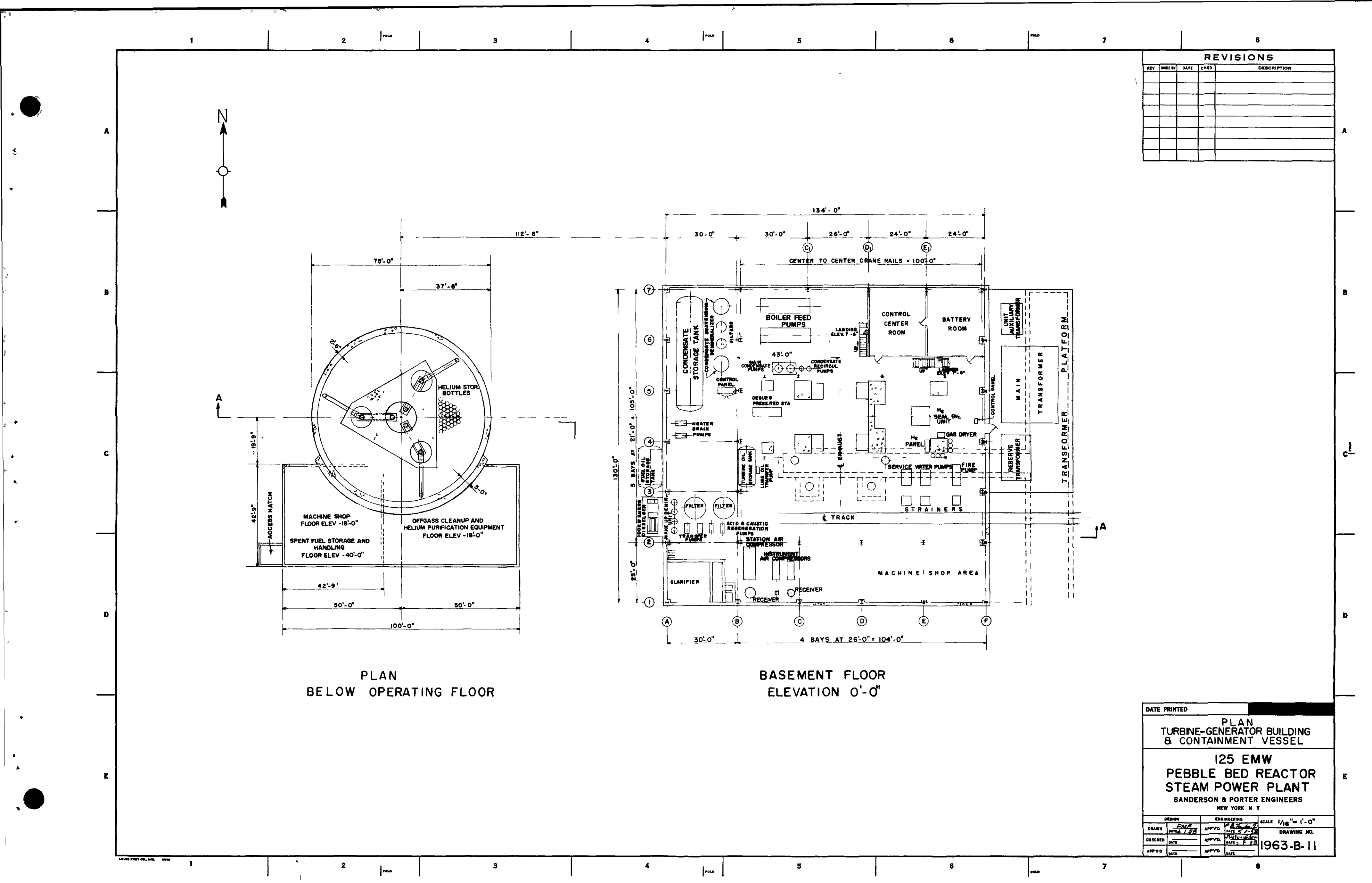




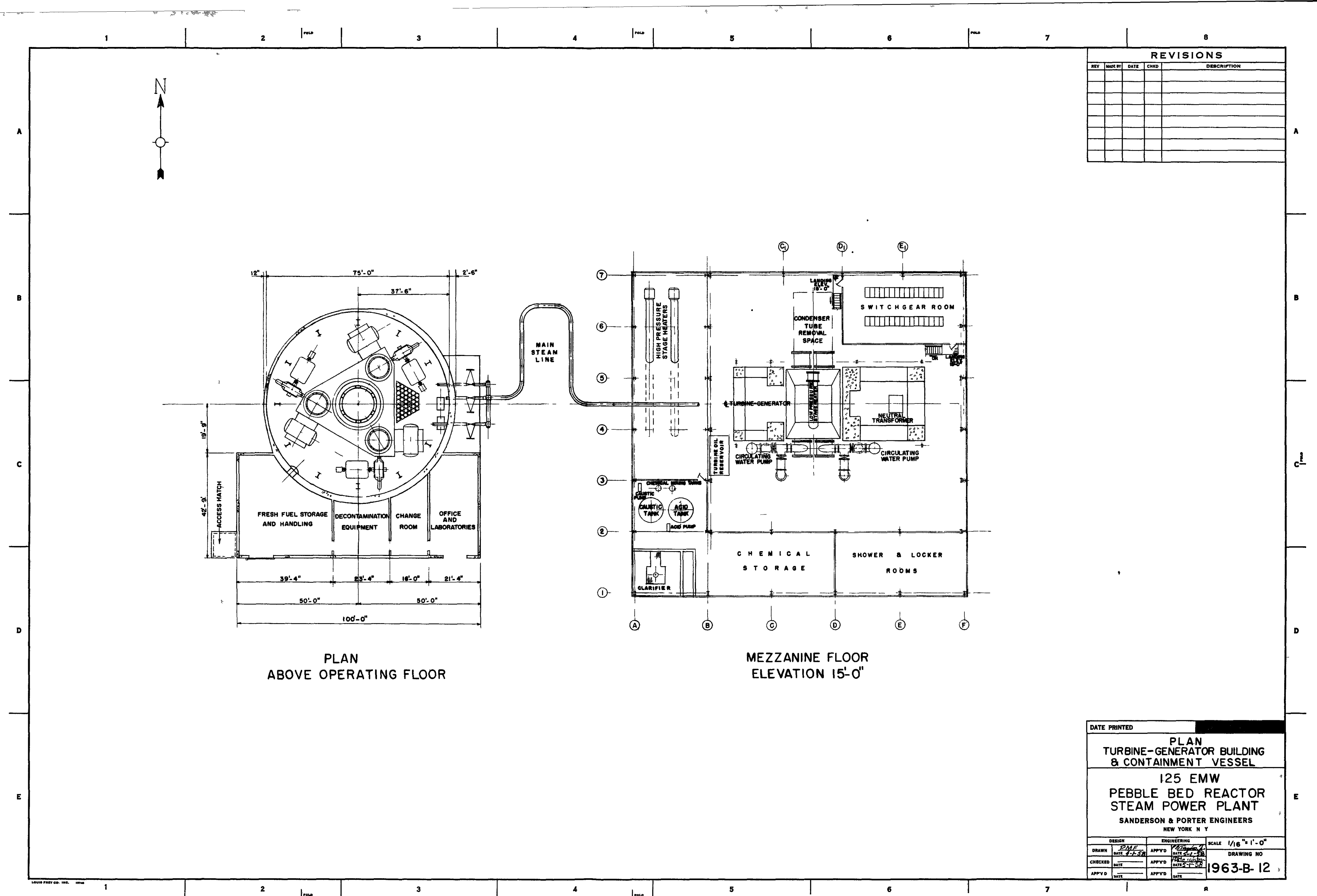




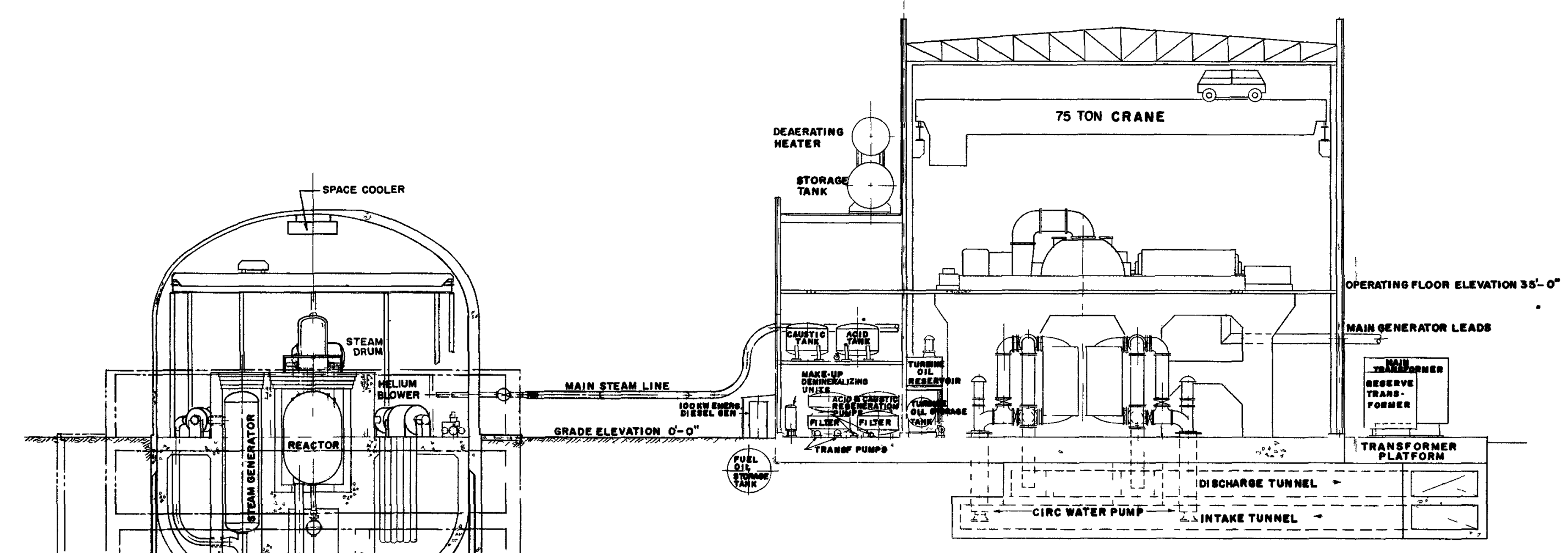

D

SECTION A-A

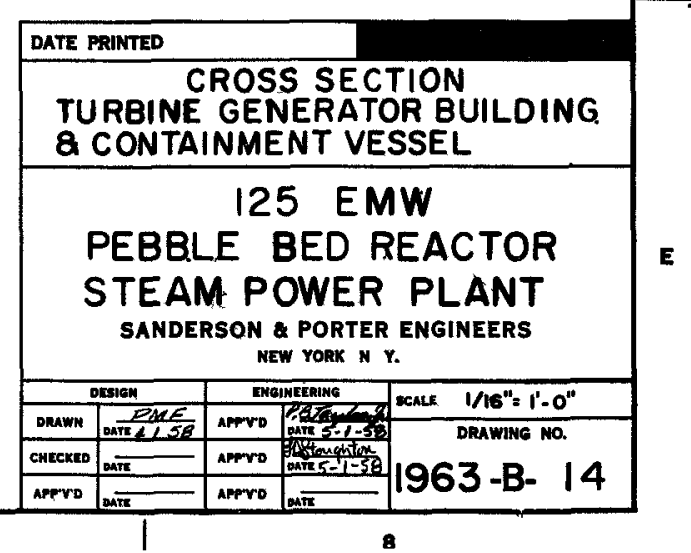




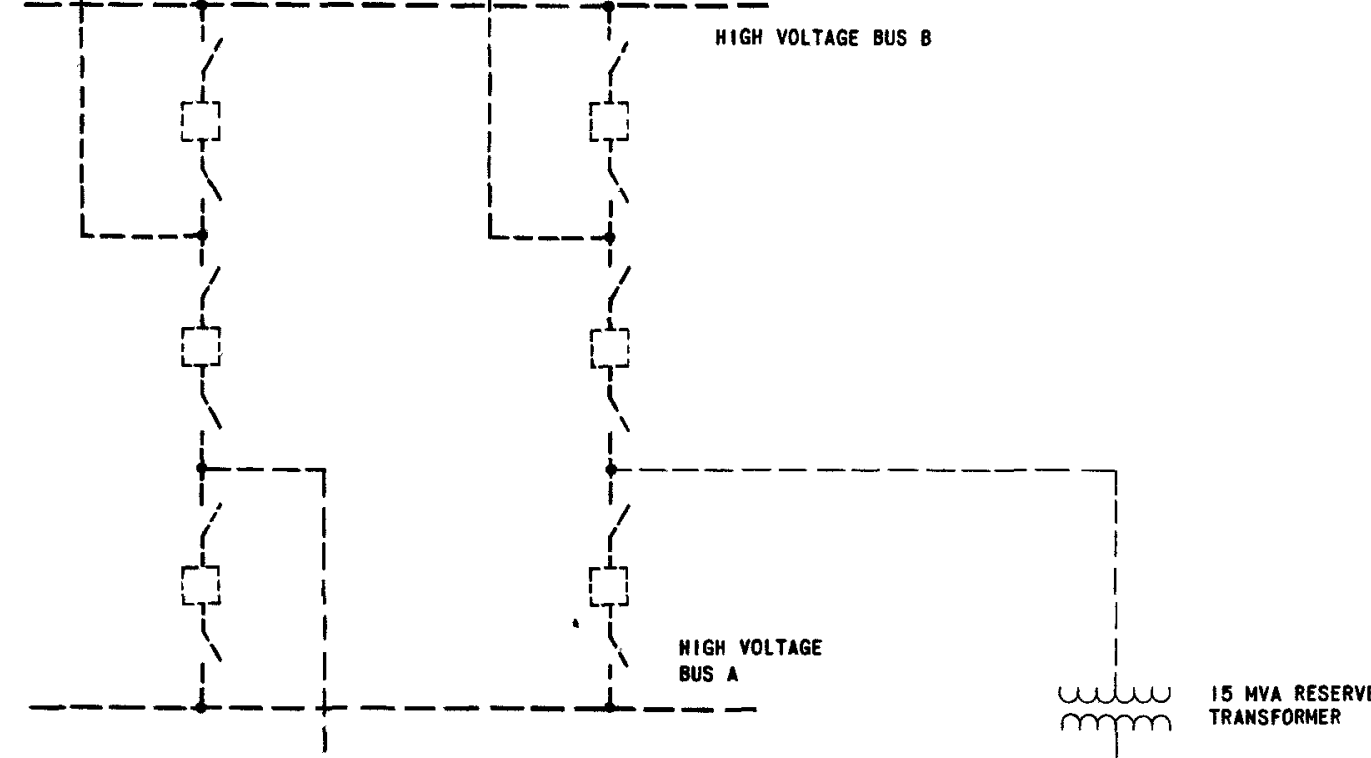

srmeols:

† OIL CIRCUIT BREARER

个

DRAN OUT CIRCUIT BREREE

ulue 140 nva man

Disconnectrmg SWITen

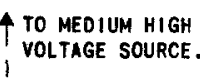

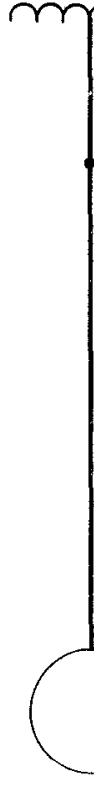

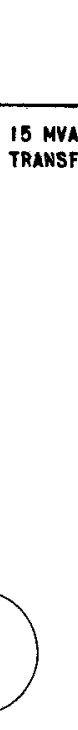

160 NVA
TURB INE GENERTOR
15KY

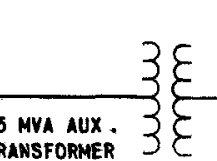
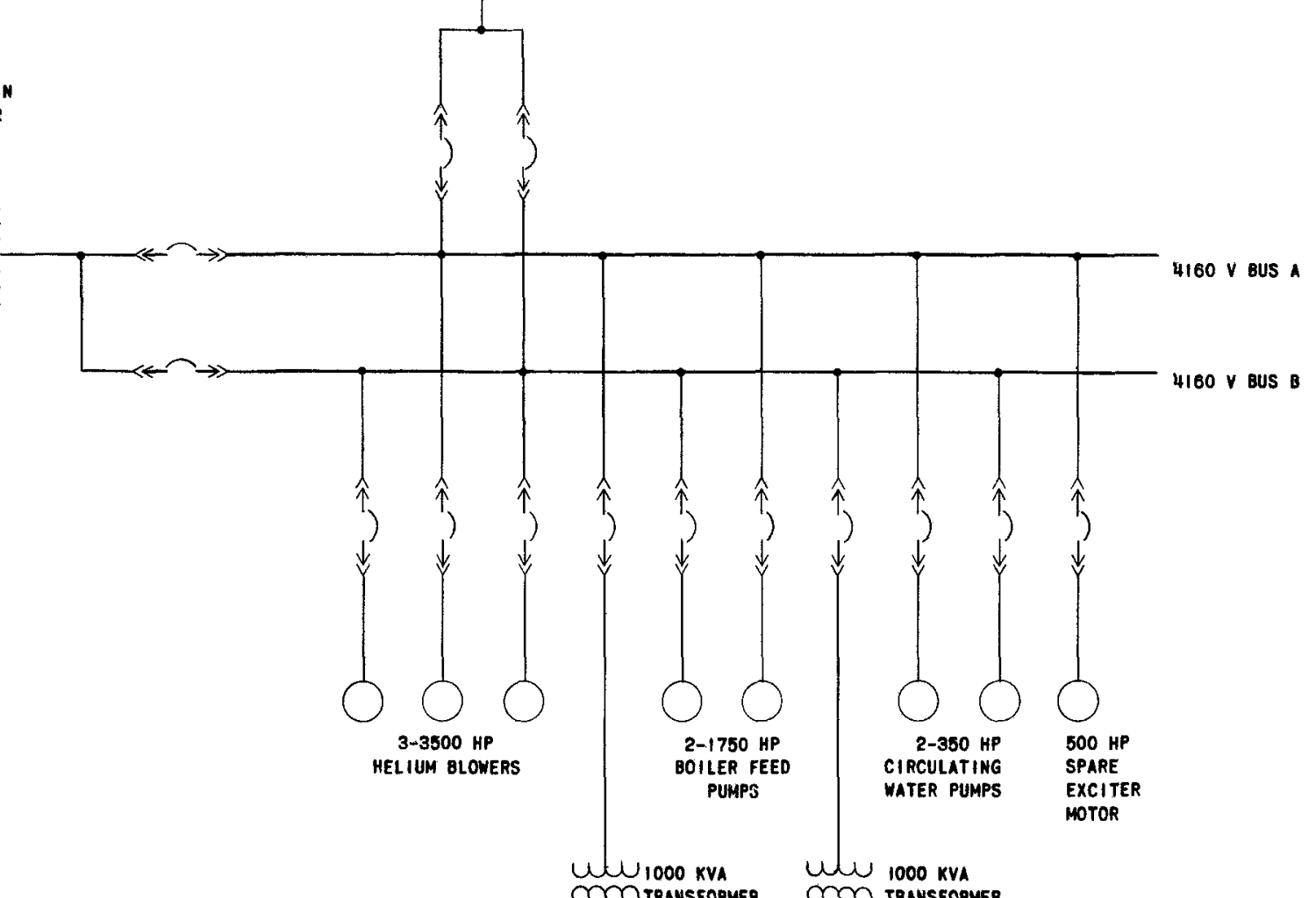

i

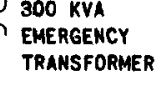
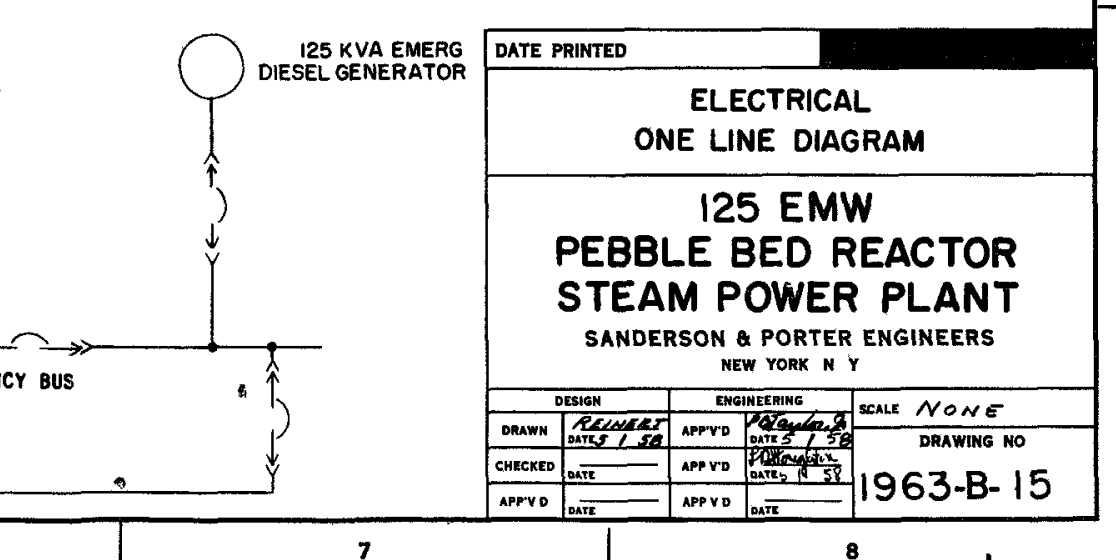


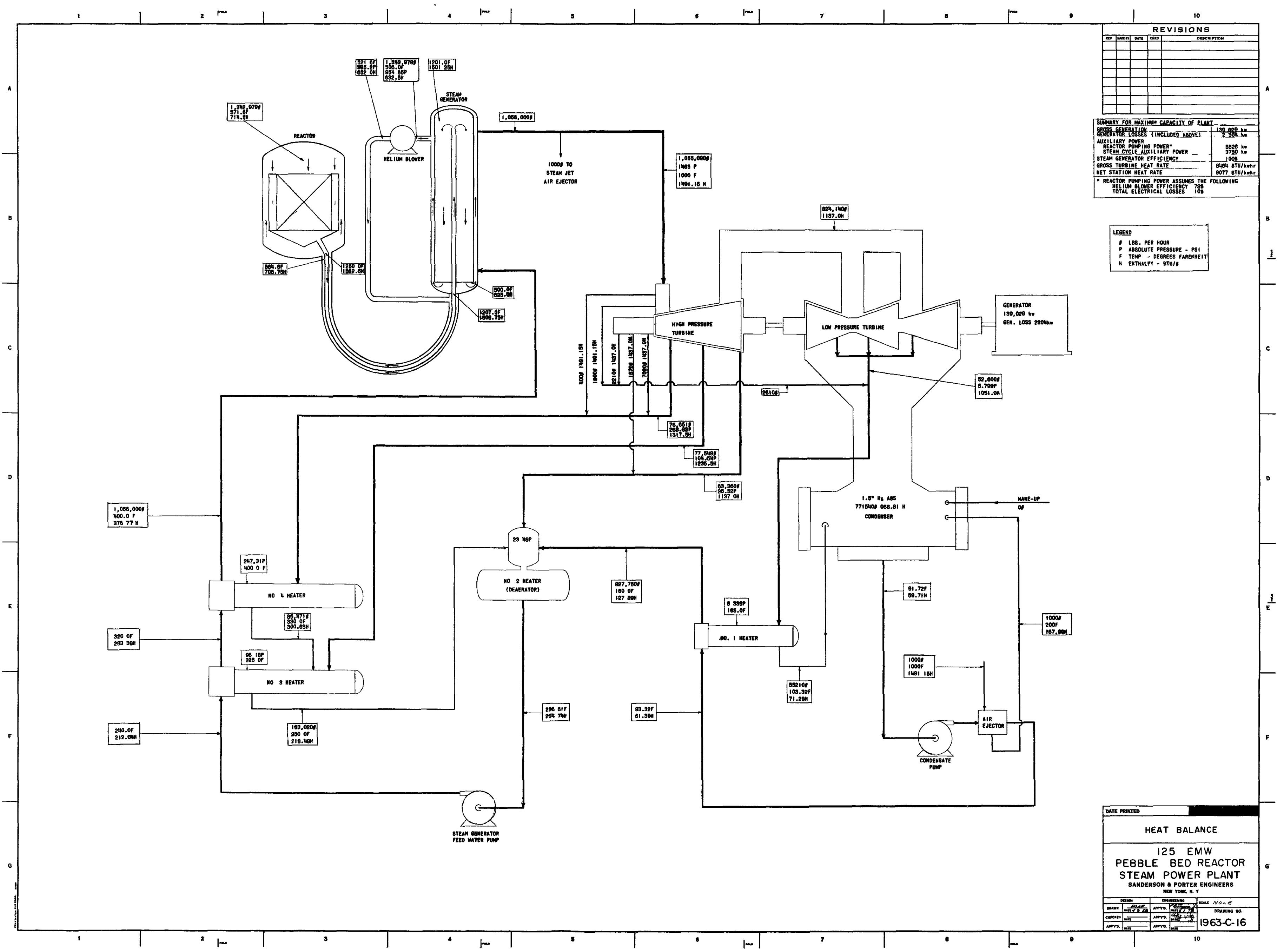




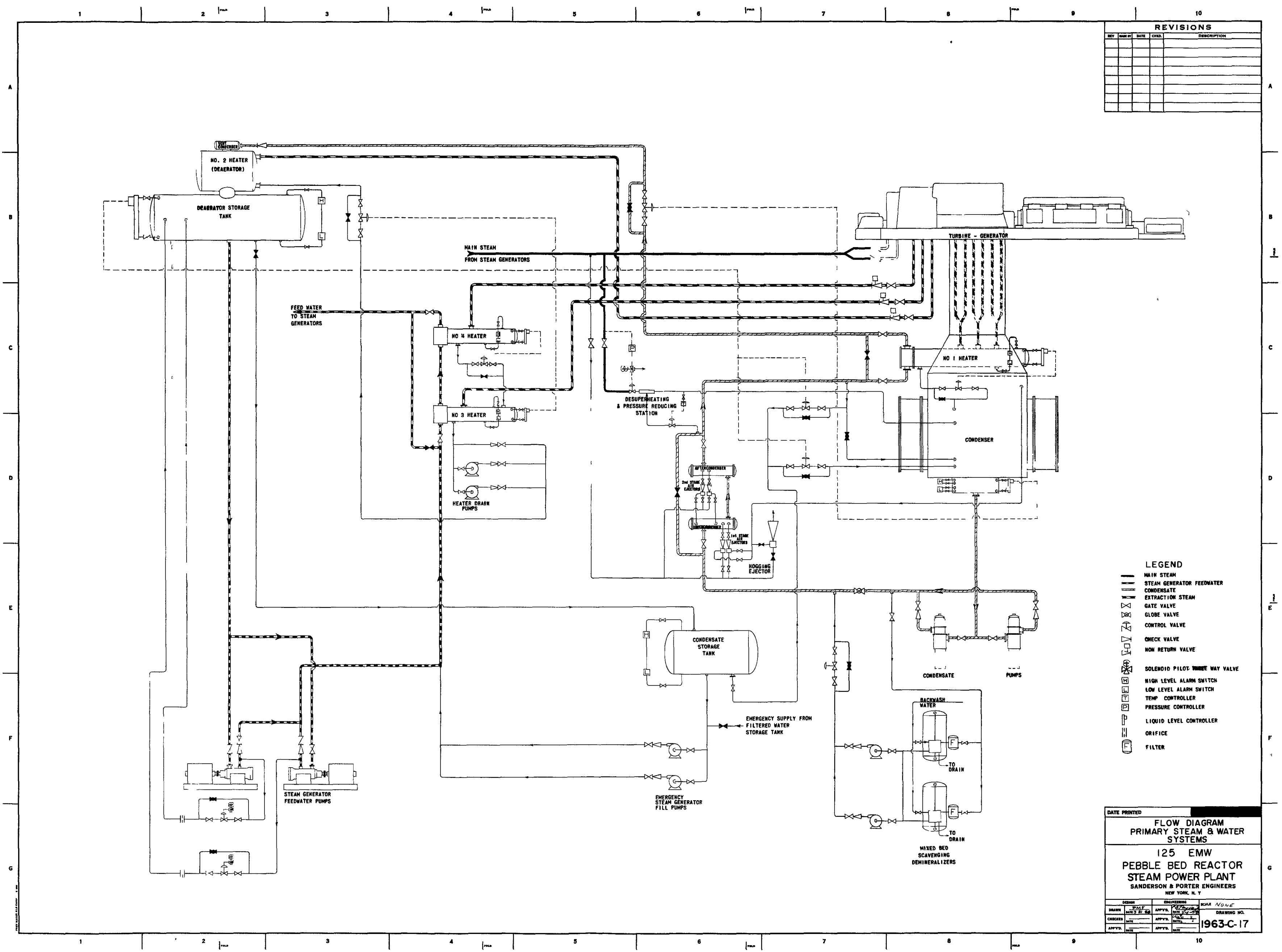




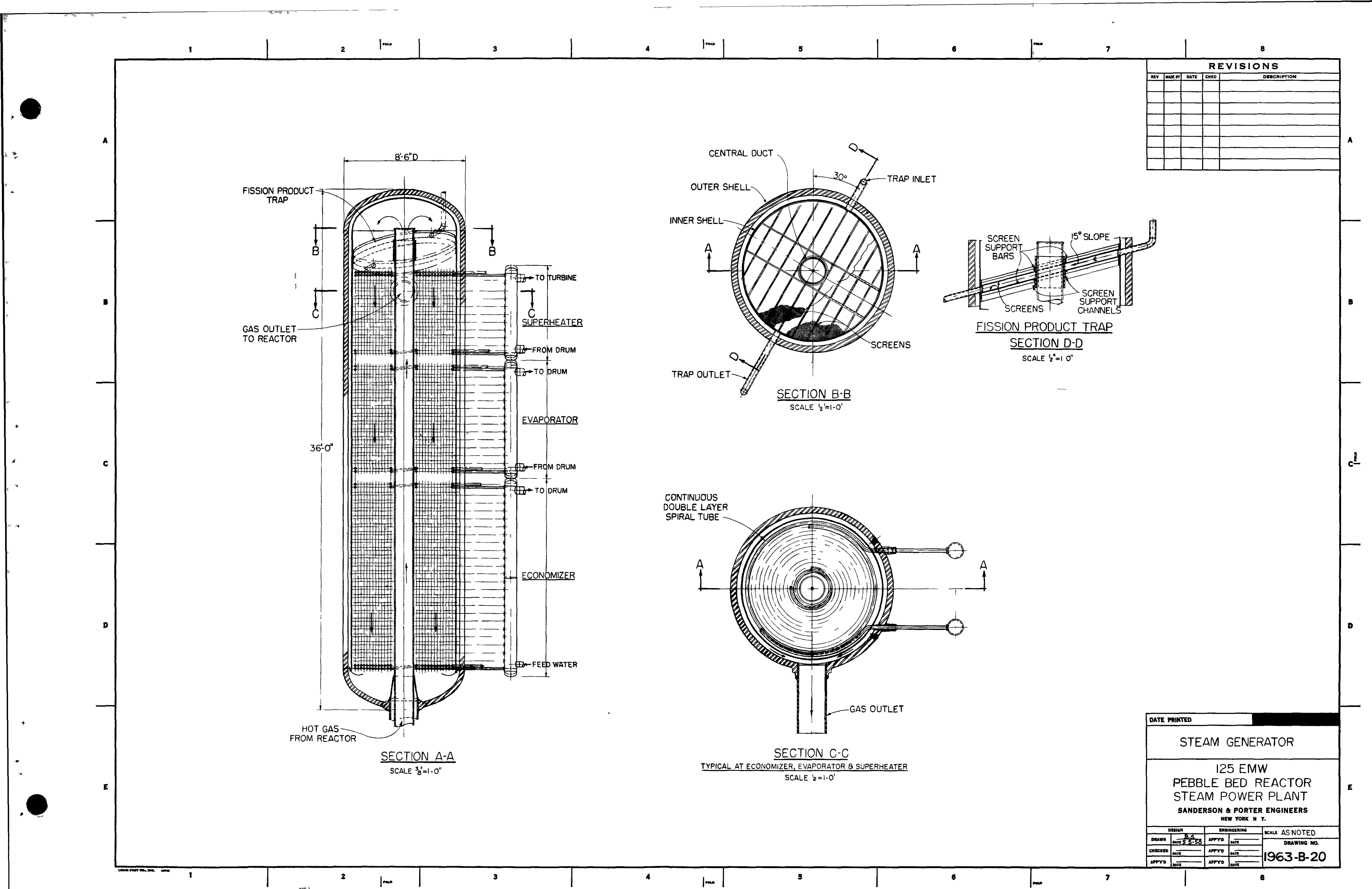




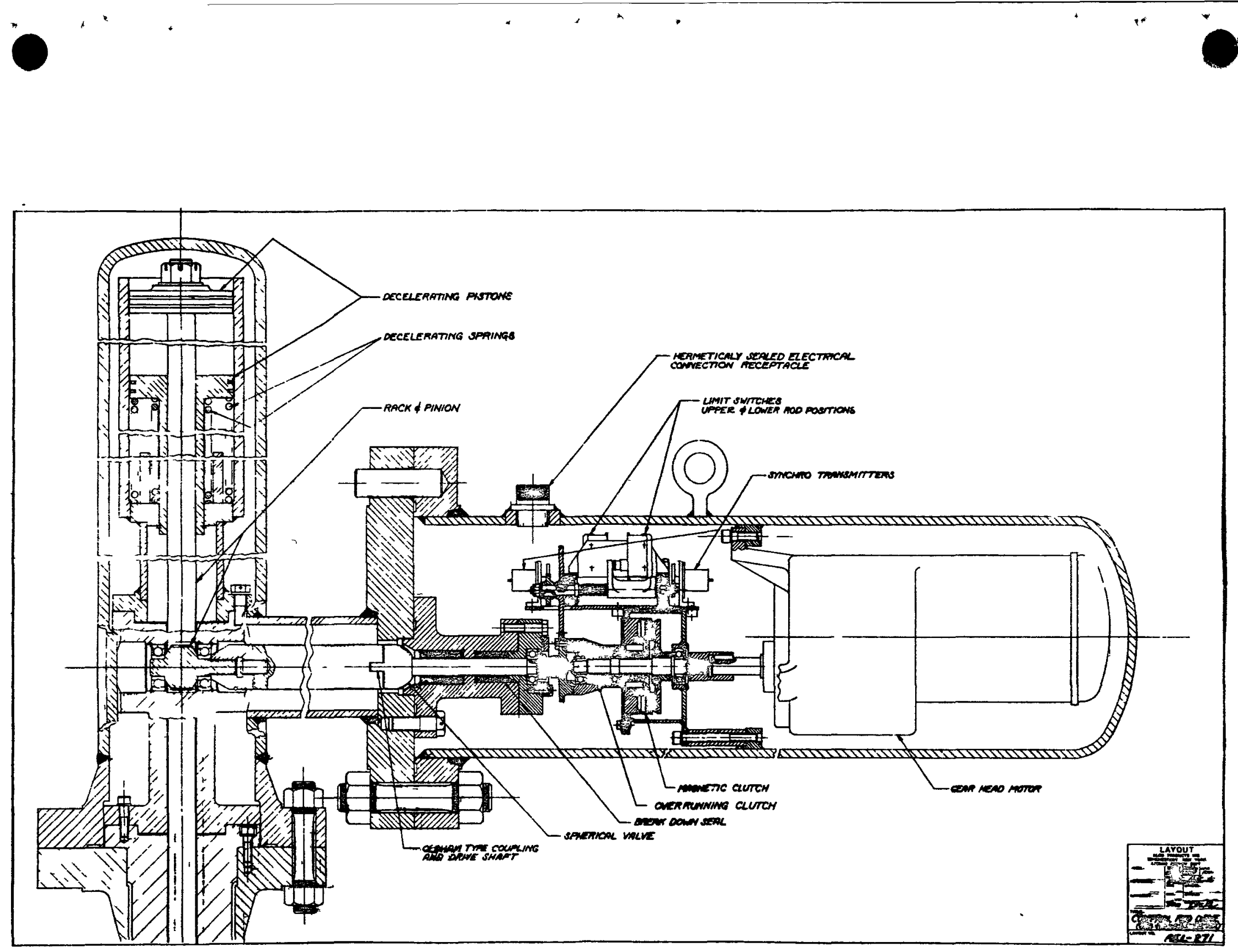




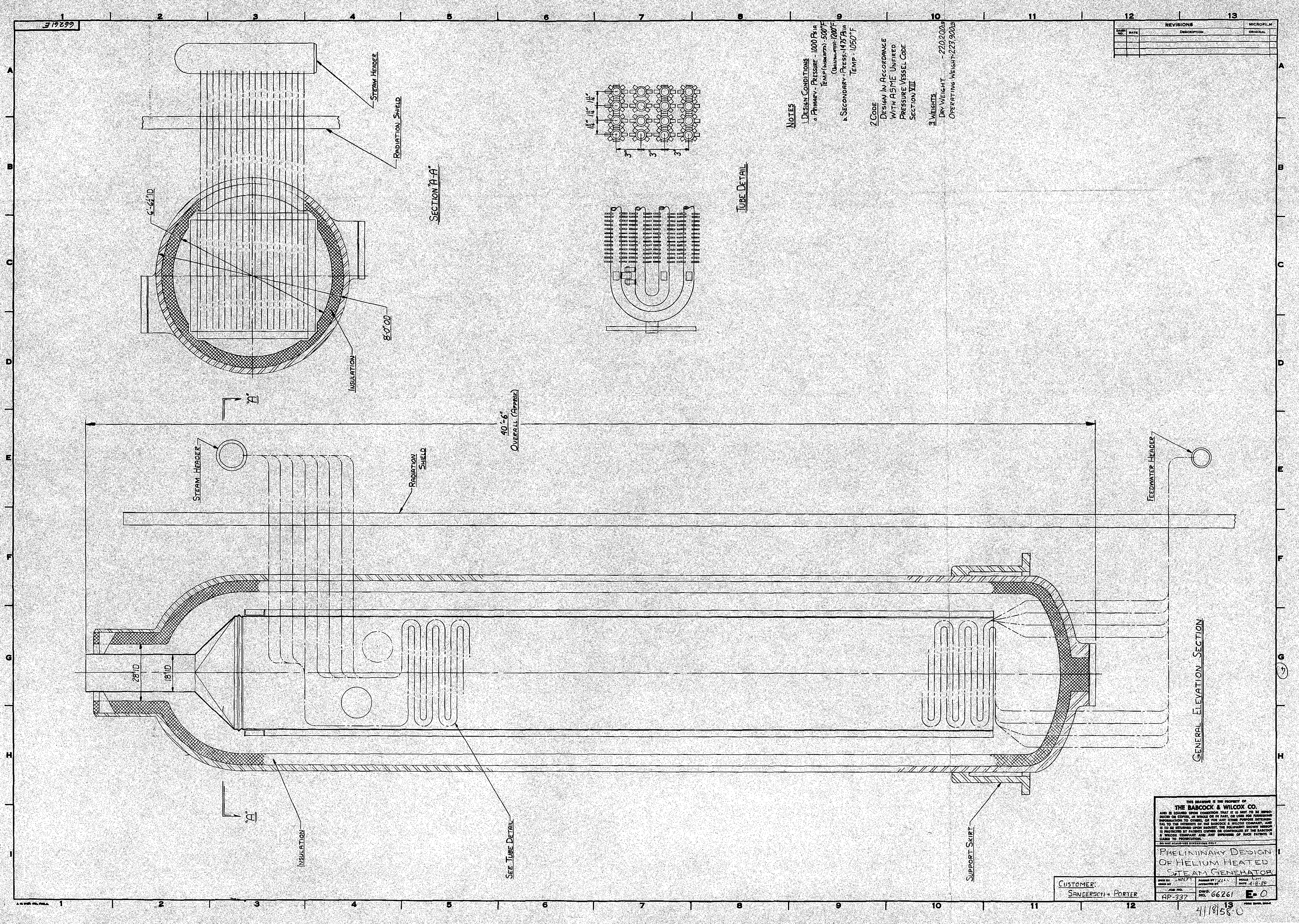




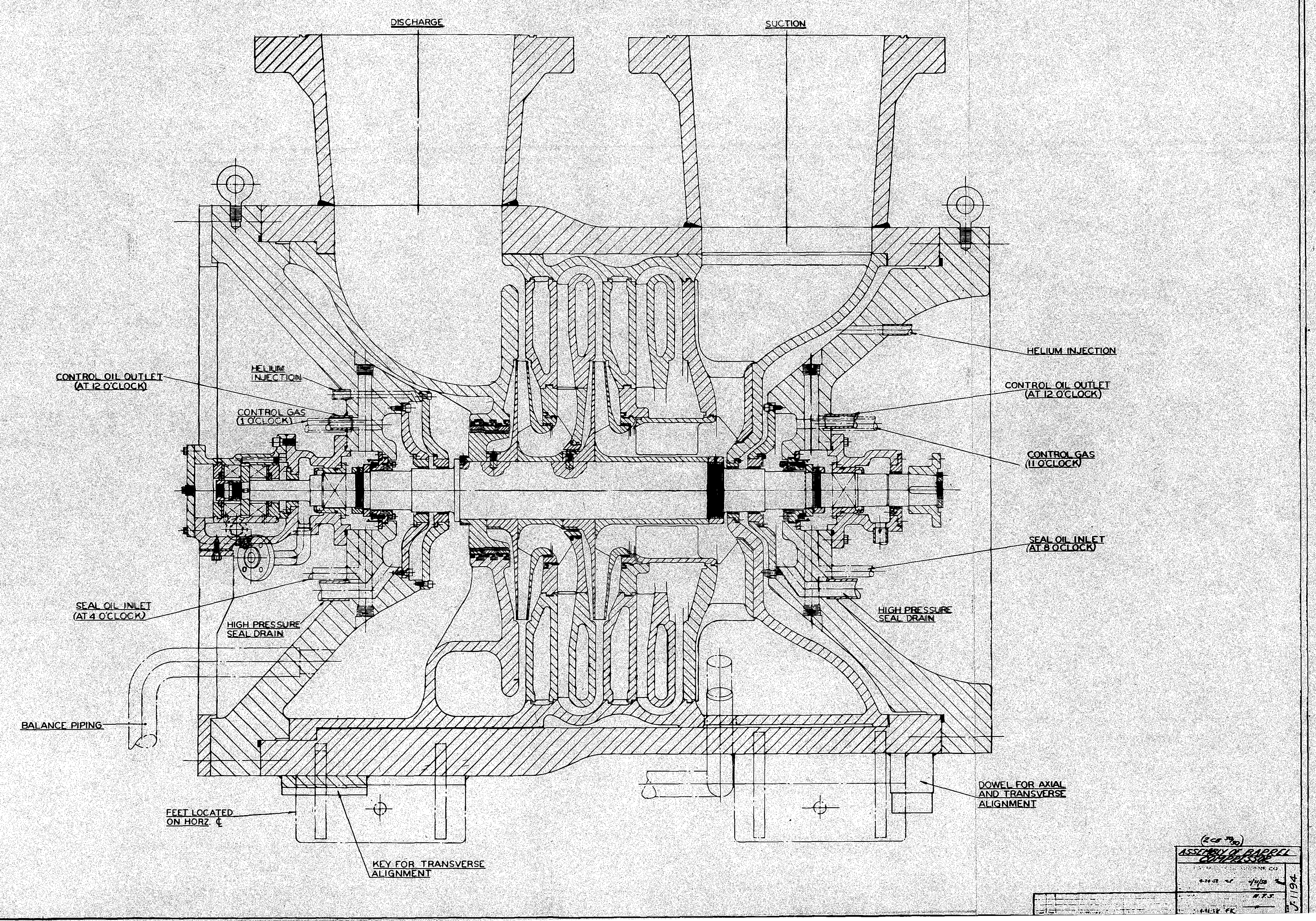




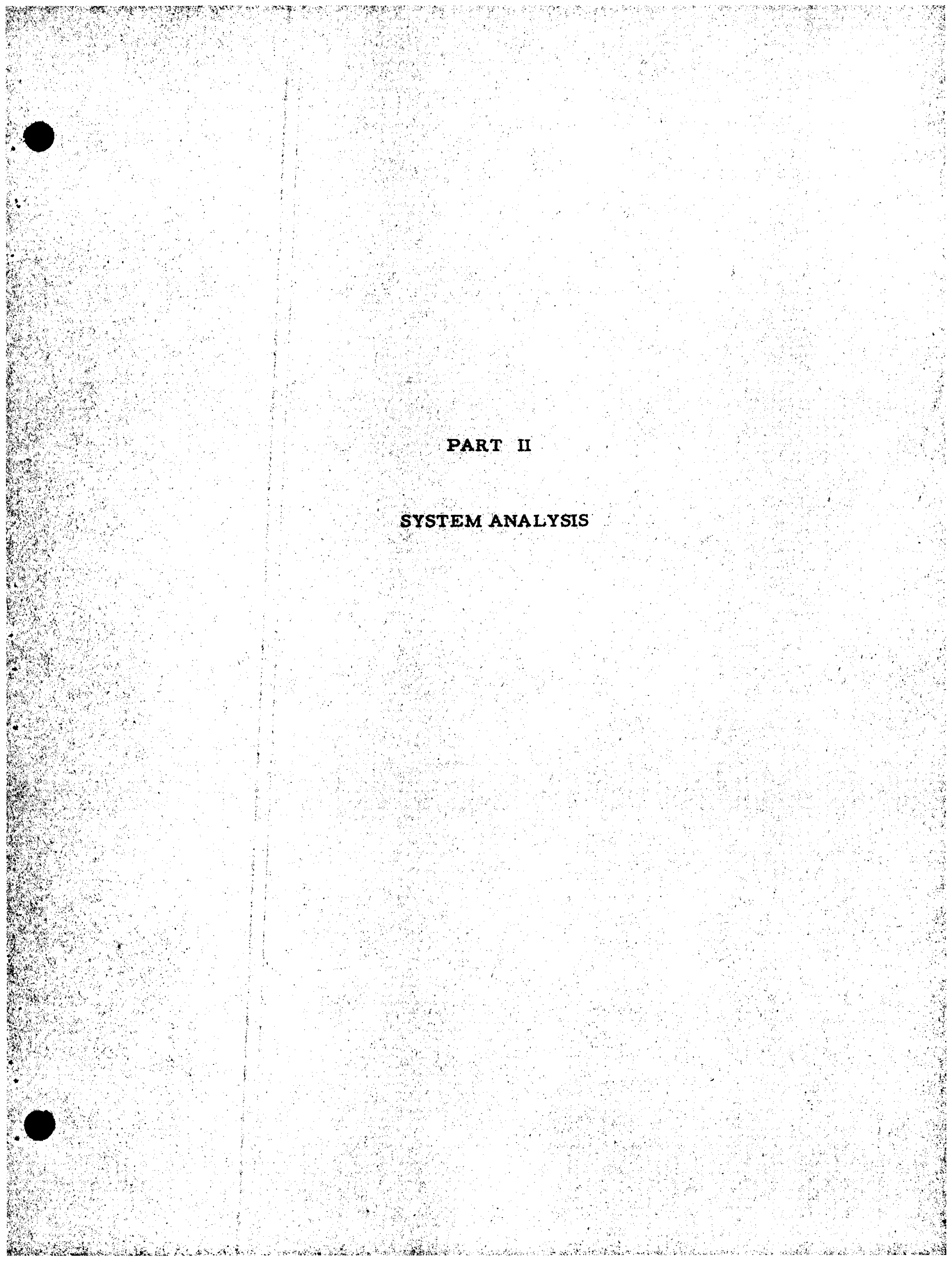


Section 1.0

Reactor Physics

Page

1.1 Core Design Philosophy $\ldots \ldots \ldots \ldots \ldots \ldots \ldots \ldots \ldots$ 1-1

1.2 Description of Parameter Survey ............ 1-1

1.2.1 Calculation Mudel .................... 1-2

1.2.1.1 Determination of Fast Constants ..... 1-3

1.2.1.2 Determination of Thermal Constants .. 1-6

1.2.2 Multi-group Cross-sections ........... 1-8

1.2.2.1 Relation of Resonance Integral and

Average Cross-section ........... 1-8

1.2.2.2 Resonance Cross-sections ......... 1-10,

1.2.2.3 Averaging Over Unresolved Resonances. 1-13

1.2.2.4 Fission Cross-sections 1-17

1.2.2.5 Theory of Average Cross-sections for

Fissionable Materials ........... 1-17

1.2.2.6 Calculations of U-233 Cross-sections.. 1-22

1.2.2.7 Evaluation of Integrals ......... 1-24

1.2.2.8 Fast Cross-section and Group

Constants ................. 1-26

1.2.3 Flux Depression and Doppler Effect ........ 1-28

1.2.3.1 Theory and Development of Equations.. 1-28

1.2.3.2 Preparation of Graphs and Tables for

Calculation of $f . \ldots \ldots \ldots \ldots \ldots \ldots, 1-32$

1.2.3.3 Calculational Procedure for Resolved

Resonances and $1 / v$ Cross-section .... 1-37

1.2.3.4 Unresolved Resonances ............ 1-39

1.2.3.5 Treatment of U-233 ........... 1-40

1.2.4 Discussion of Values of Capture to Fission Ratio

for $U-233 \ldots \ldots \ldots \ldots \ldots \ldots \ldots \ldots \ldots$ 1-44

1.2.5 Thermal Neutron Spectrum and Thermal

Properties ..................... 1-46

1.2.6 Calculation of Breeding Ratio ........... 1-49

1.2.7 Results of Nuc? ear Parameter Survey ...... 1-50

1.2.7.1 Single Region Core ............. 1-50

1.2.7.2 Small Breeder Blanket Core ......... 1-51

1.2.7.3 Large Breeder Blanket Cores ........ 1-52 


\section{Table of Contents $\left(\operatorname{con}^{\prime} t\right)$}

Page

1.3 Selection of Final Core $\ldots \ldots \ldots \ldots \ldots \ldots \ldots \ldots \ldots$ 1-65

1.4 Analysis of Final Core $\ldots \ldots \ldots \ldots \ldots \ldots \ldots \ldots \ldots, 1-70$

1.4.1 Core Loading ..................... 1-70

1.4.2 Control Rod Requirements ............. 1-70

1.4.3 Power and Flux Requirements ........... 1-71

1.4.3.1 Radial Flux and Power ........... 1-73

1.4.3.2 Axial Flux and Power ........... 1-73

1.4.4 Initial Breeding Ratio................ 1-79

1.4 .5 Buildup of Isotopes .................. 1-84

1.4.5.1 Buildup in the Core ............ 1-85

1.4.5.2 Buildup in the Blanket.......... $1-86$

1.4.6 Core Lifetime .................. $1-90$

1.4.7 Neutron Spectrum in the Core ........... 1-92

1.4.8 Temperature Coefficient of Rwactivity ....... 1-94

1.4.9 Summary of Reactor Characteristics ........ 1-96

1.5 Shielding Analysis .................... 1-98

1.5.1 Shutdown Shielding Requirements .......... 1-98

1.5.2 Primary Shielding .................. 1-98

1.5.3 Vapor Container Shielding ............... 1-102

NOMENCLATURE $\ldots \ldots \ldots \ldots \ldots \ldots \ldots \ldots \ldots \ldots \ldots \ldots \ldots$ 1-106

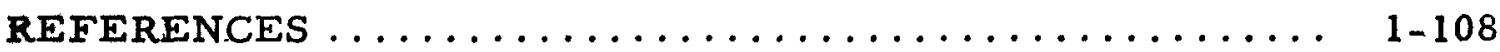




\subsection{Reactor Physics}

\section{1. l Core Design Philosophy}

In the initial planning of the nuclear calculations, it was unknown whether a large single region core or a smaller core with a fertile blanket would be the most desirable. It was realized that a large core would require a field fabricated vessel and a lower coolant operatin pressure. The smaller core might possibly be shop fabricated and certainly more readily transported, but would involve a more complicated mechanical design due to its two regions. With the two opposing concepts in mind it was decided to investigate a series of core diameters ranging from 7 to $20 \mathrm{ft}$. The $7 \mathrm{ft}$. core was surrounded with a fertile blanket to supplement its lower conversion ratio and make it more comparable with the larger cores.

Economics, in the form of fuel cost and pumping power, were also deemed important. Therefore, to consider them adequately, it was necessary to conduct a nuclear parameter survey in order to present a scries of cores which werc acceptable from the physics aspect. These cores could then be compared and a more nearly optimum design selected. This was done and is explained in detail.in Sections 1.2 and 1. 3 .

Following the selection of the final design, a more detailed analysis was conducted to determine its characteristics.

\subsection{Description of the Parameter Survey}

The initial calculations done on the pebble bed reactor were designed to cover a wide range of parameters in order to give an overall picture of the effect of the many variables. The following table lists the variables and the range considered.

TABLE 1.2-1

Variable

Weight per cent total oxide in fuel elements Average void content of the core

Core diameter

$\mathrm{Th} / \mathrm{U}$ atom ratio
Range

7. 5-30\%

$10-39 \%$

$7-20 \mathrm{ft}$.

$5-50$ 
The core was assumed to be composed of a homogenized mixture of $\mathrm{ThO}_{2}, \mathrm{U}^{233} \mathrm{O}_{2}, \mathrm{C}$, and void. The concentration of the components was based on the fuel, graphite and fertile material being in the form of spheres and thus having an associated voidage of $39 \%$. Cases where the void was less than $39 \%$ of the core volume, the reduction was achieved by replacing a portion of the fuel balls with solid graphite. Since the core was homogenized, the effect of lumping the graphite does not appear in the results.

The average moderator temperature was assumed to be $1200^{\circ} \mathrm{F}$ with the reactor operating at $350 \mathrm{MW}$. It is expected to vary with core size and void volume, but in a survey where numerous cores are considered, a detailed calculation of average moderator temperature for each is not feasible. Small temperature variations of the order of $100^{\circ} \mathrm{F}$ would not change the calculated reactivity by more than 0.3 percent as noted by the temperature coefficient.

The effect of xenon on initial reactivity was accounted for by calculating the equilibrium xenon concentration from the average flux at full power operation. The xenon was assumed to remain in the fuel elements for purposes of this calculation, although some will probably diffuse into the coolant gas. Because of the lack of detailed temperature distributions and conflicting diffusion constants any diffusion calculation was considered unreliable at this time.

\subsection{Calculation Mode1}

In order to make an extensive survey of various reactor core compositions, it was necessary to employ a relatively simple nuclear model which would be expected to give reasonable accurate values of $\mathrm{K}_{\mathrm{eff}}$. Due to appreciable resonance absorption in the core, at least three neutron groups should be used to describe the neutron flux. How ever, the three group may be reduced to two with multiplication in the fast group with little loss of generality. The general two group equations, including resonance fission and absorption are:

$$
\begin{aligned}
& -D_{1} \nabla^{2} F(\underline{r})+\sum_{1} F(\underline{r})=S(\underline{r}) \\
& -D_{2} \nabla^{2} \phi(\underline{r})+\sum_{a_{2}} \phi(\underline{r})=p \sum, F(\underline{r})
\end{aligned}
$$


where the source term is defined as:

$$
S(r)=\left[(1-p) k_{f} \sum, F(\underline{r})+k_{t h} \sum a_{2} \phi(r)\right] \frac{V_{c}}{V}
$$

In the special case of a bare reactor:

$$
\begin{aligned}
& \nabla^{2} F(\underline{r})=-B^{2} F(\underline{r}) \\
& \nabla^{2} \phi(r)=-B^{2} \phi(\underline{r})
\end{aligned}
$$

Solving the above set of equations to determine the expression for $\mathrm{K}_{\mathrm{eff}}$ yields:

$$
k_{\text {eff }}=\frac{V}{V_{c}}=\frac{p k_{t h}}{\left(1+L^{2} B^{2}\right)\left(1+\tau B^{2}\right)}+\frac{(1-p) k_{f}}{\left(1+T B^{2}\right)}
$$

which is the standard modified two-group equation.

The effect of a reflector was included by modifying the definition of the buckling:

$$
B^{2}=\left[\frac{\pi}{H+2 S_{z}}\right]^{2}+\left[\frac{4.810}{D+2 S_{R}}\right]^{2}
$$

where $S_{z}$ is the axial reflector savings and $S_{R}$ is the radial reflector savings.

\subsubsection{Determination of Fast Constants}

In order to obtain the fast constants with a reasonable degree of accuracy the Fourier transform of the slowing down distribution from a point source in space was used (1)*.

* Numbers in parentheses designate references on page 1-10.8. 
In a non-hydrogeneous medium, neglecting inelastic scattering, the first Legendre coefficients of the flux, known as the P-1 approximation, are determined from the following set of equations:

$$
\begin{aligned}
& \sum_{a}(u) F_{c}(u)-i B F_{1}(u)=f_{0}(u)-\frac{\partial q}{\partial u} \\
& \gamma_{(u)} \sum_{(u)}-\sum_{i}(\bar{u}) ; \sum_{s_{0}}^{i} F_{1}(u)=i \frac{B}{3} F_{0}(u) \\
& \sum_{i} \xi^{i}(u) \sum_{s_{0}}^{i}(u) F_{0}(u)-q(u)=0
\end{aligned}
$$

where the term $\gamma(u)$ is defined as :

$$
\gamma(u)=\frac{\alpha^{2} \tan ^{-1} \alpha}{3\left(\alpha-\tan ^{-1} \alpha\right)} ; \alpha \frac{B}{\sum(u)}
$$

The solution of the above equations was facilitated through the use of a machine code called MUFT III and described in detail in reference (1). In brief, the code calculates the average flux $\left(F_{0}\right)$, ourrent $\left(-i F_{1}\right)$ and the slowing down (q) in each of the 54 or 59 groups depending on where the fast cutoff* was taken. These values are then used in conjunction with the appropriate cross-sections to determine the few group (1, 2, 3,5 and 7 groups) constants as outlined below. The superscript indicates the number of groups and the subscript indicates the group number.

$$
\begin{aligned}
& \Gamma_{m}^{7}=\sum_{k<n \leq l} \bar{F}_{n} \Delta_{n} \quad \text { where m }=(1,2,3 \ldots-7) \\
& \Lambda_{m}^{7}=\sum_{k<n \leq l} \bar{J} \Delta_{n}
\end{aligned}
$$

* 54 groups correspond to $1200^{\circ} \mathrm{F}$ and 59 groups correspond to $77^{\circ} \mathrm{F}$. 


$$
\begin{aligned}
& \vec{\Xi}_{m}^{7}=\sum_{k<n \leq l}\left[\sum_{c_{n}} \bar{F}_{n} \Delta_{n}+q_{n-1}+\bar{\Sigma}_{f_{n}} \bar{F}_{n} \Delta_{n}\right] \\
& \alpha_{m}^{7}=\sum_{k<n \leq l} \bar{T}_{n} \bar{F}_{n} \Delta_{n}
\end{aligned}
$$

The few group constants can be transposed into a more familiar and useable form by the following definitions:

$$
\begin{aligned}
& D_{m}^{\prime}=\frac{\Lambda_{m}}{B \Gamma_{m}} \\
& \sum_{m}^{7}=\frac{q_{l+} \Xi_{m}}{\Gamma_{m}} \\
& \sum_{a_{m}}^{7}=\frac{\Xi_{m}}{\Gamma_{m}} \\
& v \sum_{f m}^{7}=\frac{\alpha_{m}}{\Gamma_{m}} \\
& p_{m}^{7}=\frac{\sum_{m}^{7}-\sum_{a_{m}}^{7}}{\sum_{m}^{7}} \\
& \Gamma_{m}^{7}=\frac{\Lambda_{m}^{7}}{B\left(q l+\Xi_{m}\right)}
\end{aligned}
$$

The seven fast group constants can be coalesced into the one fast group value, necessary for the modified two-group calculations, by utilizing the following expressions: 


$$
\begin{aligned}
& \alpha_{1}^{1}=\alpha_{1}^{2}+\alpha_{2}^{2} \\
& \Gamma_{1}^{1}=\Gamma_{1}^{2}+\Gamma_{2}^{2} \\
& \Lambda_{1}^{1}=\Lambda_{1}^{2}+\Lambda_{2}^{2} \\
& \varpi_{1}=\varpi_{1}+\varpi^{2} \\
& \varpi_{1}
\end{aligned}
$$

The above equations illustrate the coalescence from two to one fast

\begin{tabular}{|c|c|c|c|c|}
\hline Seven Groups & Five Groups & Three Groups & Two Groups & One Group \\
\hline $\mathrm{m}=1$ & 1 & & & \\
\hline 2 & 2 & & & \\
\hline 3 & 3 & 1 & & \\
\hline 4 & & & & \\
\hline 5 & 4 & 2 & 1 & \\
\hline 6 & & & & \\
\hline 7 & 5 & 3 & 2 & 1 \\
\hline
\end{tabular}
group. The procedure is identical in going from seven to five and five to three only the subscripts and superscripts would differ. The overall coalescence scheme is shown in the following diagram.

\subsubsection{Determination of Thermal Constants}

In calculations involving the breeding ratio, it was desired to poison out the core until $\mathrm{K}_{\text {eff }}$ was unity in order to account for parasitic captures in the control rods. The rods are principally thermal neutron absorbers, therefore a total thermal neutron absorption cross-section for the core was determined by:

$$
\sum_{a}^{T^{\prime}}=\frac{V \sigma_{f}^{23} N^{23}}{\frac{\left(1+\tau B^{2}\right)}{p}-\frac{(1-p) k_{f}}{p}}-D_{s} B^{2}
$$


where the prime denotes the value for a just critical reactor.

The coupling coefficient between the fast and thermal flux was calculated using the total thermal absorption cross-section from above,

$$
\frac{\bar{\phi}_{f}}{\bar{\phi}_{t h}}=\frac{\left[D_{s} B^{2}+\sum_{a}^{T^{\prime}}\right] J}{p D_{f}}
$$

Thus neutron fluxes reflect the control rod absorptions.

The average thermal neutron flux was determined through use of the known power output of the core.

$$
\Phi_{t h}=\frac{C[\text { Reactor Power }]}{\left\{\sum_{f_{t h}}^{23}+\left[\Phi / \Phi_{t h}\right] \sum_{f f}^{23}\right\} \text { Reactor Vol }}
$$

The conversion factor $\mathrm{C}=3.121 \times 10^{16} \mathrm{fissions} / \mathrm{MW}-\mathrm{sec}$ was obtained by assuming $200 \mathrm{mev}$ of energy released per fission

The effect of the fission product xenon was included in the calculations by considering the equilibrium concentration as

$$
N^{x e}=\frac{[0.059]\left\{\sum_{f t h}^{23}+\bar{\phi}_{f /} \bar{\phi}_{t h}\left(\sum_{f f}^{23}\right) ; p_{t h}\right.}{\left[2.1 \times 10^{-5}+\sigma \times e \bar{\phi}_{t h}\right]}
$$

The effective multiplication constant was calculated both with and without equilibrium xenon. Where it was included, the total thermal absorption cross-section was modified as follows:

$$
\sum_{a}^{T^{\prime \prime}}=\sum_{a}^{T}+N^{x e} \bar{\sigma}_{x e}
$$

where the double primes denote equilibrium xenon.

Due to significant thermal absorption, the following definition of the thermal diffusion coefficient, $D_{s}$, was used:

$$
D_{s}=\frac{\sum_{s}+9 / 5 \sum_{a}^{T}}{3\left[\sum_{s}+\sum_{a}^{T}\right]^{2}}=D_{2}
$$


The appropriate absorption cross-section was used, depending on whether equilibrium xenon was considered.

Through use of the terms presented above and in Section 1.2.1.1 it is possible to determine most of the parameters of interest in the survey.

\subsubsection{Multigxoup Cross-sections (2)}

The multigroup cross-sections for Th-232 and U-233 were originally calculated for 13 fast groups extending from $10 \mathrm{Mdv}$ to $0.196 \mathrm{ev}$. These were then recalculated in order to divide them into 18 groups so each group lethargy width would not be over unity. Subsequent use of the MUFT III slowing down code revealed it desirable to use a much smaller lethargy width; thus, the 18 group cross-sections were split up into 59 groups.

The method used to determine the original 13 fast group cross sections is given below, The 18 group values are listed at the end of this section.

\subsubsection{Relation of Resonance Integral and Average Cross-section}

Epithermal neutron absorption in homogeneous mixtures of dilute fissionable or fertile material with moderator may be simply represented by use of the resonance integral, $\int \sigma_{\alpha} \frac{d E}{E}$. According to Fermi's model of slowing and capture for an infinite medium,

$$
\frac{d q}{d u}=-\phi \sum_{a}
$$

If the absorption cross-section does not seriously disturb the flux,

$$
\phi \simeq \frac{q}{\xi \Sigma_{s}}
$$

Thus

$$
\frac{d q}{d u}=-\frac{q \sum a}{\xi \sum s}
$$


and integrating

$$
q=q_{0} \exp \int_{u_{0}}^{u}-\frac{\sum_{a} d u}{\xi \sum_{s}}
$$

If it is assumed that an average value of $\xi \Sigma_{s}$ can be formed for a given range of lethargy, then the resonance escape probability is,

$$
p=\frac{q}{q_{0}}=\exp -\frac{N_{a} \sigma_{r}}{\bar{\xi} \bar{\Sigma}_{s}}
$$

where

and

$$
\mathrm{N}_{\mathrm{a}}=\text { number of absorbing nuclei per unit volume }
$$

$$
\sigma_{r}=\int_{u_{0}}^{u} \sigma_{a} d u
$$

is the resonance integral.

The multigroup counterpart of the differential equation (1.2-33) is:

$$
\int_{u_{i}}^{u_{i+1}} \frac{d q}{d u} d u=-\int_{u_{i}}^{u_{i+1}} \frac{q \sum_{a d u}}{\xi \sum_{s}}
$$

where $u_{i}$ and $u_{i+1}$ are the lethargy boundaries of a given group.

Taking $\bar{\Sigma}_{\mathrm{a}}, \xi \Sigma_{\mathrm{s}}$ and $\overline{\mathrm{q}}$ as appropriate averages over the group of width $u_{(i+1)}-u_{i}=\Delta_{u}$, the integration in equation $(1.2-37)$ gives

$$
q_{(i+1)}-q_{i}=-\Delta u \frac{\bar{q} \bar{\Sigma} a}{\bar{\xi} \sum_{s}}
$$

The average group microscopic absorption cross-section is: 


$$
\bar{\sigma}_{a}=\frac{\int_{u_{i}}^{u_{i+1}} \sigma_{a} d u}{\Delta_{u}}=\frac{\sigma_{r}}{\Delta_{u}}
$$

Thus the evaluation of the piecewise resonance integral immediately yields the cross-section for the multigroup formulation.

\subsubsection{Resonance Cross-sections}

The resonance capture cross-section for interaction of neutrons of energy $E$ with nuclei is:

$$
\sigma_{c}(E)=\sigma_{0} \frac{\Gamma \gamma}{\Gamma} \psi
$$

where $\sigma_{O}$ is the peak total cross-section at energy $E_{O}, \Gamma_{\gamma}$ is the capture half width of the resonance, $\Gamma$ total half width, and $\psi$ is a Doppler correction factor. For resonance lying within a group, the resonance integral for capture is approximately:

$$
\sigma_{r}=\int \sigma_{c}(E) \frac{d E}{E}=\int_{-\infty}^{\infty} \sigma_{0} \frac{\Gamma \gamma}{\Gamma} \psi d u=\frac{\pi \sigma_{0} \Gamma_{\gamma}}{2 E_{0}}
$$

Now

$$
\sigma_{0}=\frac{2.6 \times 10^{6} \Gamma_{n} g}{E_{0} \Gamma}
$$

where $\Gamma_{n}$ is the neutron half width and $g$ is a statistical weight factor.

Combining equations $(1.2-41)$ and $(1.2-42)$,

$$
\sigma_{r}=\frac{4.09 \times 10^{6} \Gamma_{n} \Gamma_{\gamma g}}{E_{0}^{2} \Gamma}
$$


TABLE 1.2-2

RESOLVED RESONANCE CAPTURE INTEGRALS

FOR FERTILE MATERIALS

Th-232

$\mathrm{E} \quad(\mathrm{ev})$

22.0

23.7

59.6

70.1

114

123

131

152

174

195

202

215

226

235

256

269

290

310 $\sigma_{r}$ (barns)

15.84

23.55

4.572

14.27

2. 360

3.842

1.918

1.770

2.837

1.613

1.166

0.191

1. 387

0.093

1.179

0.717

0.956

0.993 
TABLE $1.2-3$

GROUP CAPTURE CROSS-SECTION DUE TO RESOLVED RESONANCES AND 1/v ABSORPTION IN Th-232

\begin{tabular}{|c|c|c|c|c|}
\hline Lethargy Range & $\begin{array}{c}\text { Group } \\
\text { Nuriser } \\
\end{array}$ & Resolved & $\begin{array}{c}\sigma \mathrm{c}(\mathrm{Th}-232) \\
1 / \mathrm{v} \\
\end{array}$ & $\underline{\text { Sum }}$ \\
\hline Thermal & & - & 6.605 & 6.605 \\
\hline $17.75-17.00$ & 13 & - & 2.235 & 2.235 \\
\hline $17.00-16.00$ & 12 & - & 1.448 & 1.448 \\
\hline $16.00-15.00$ & 11 & - & 0.880 & 0.880 \\
\hline $15.00-13.75$ & 10 & - & 0.505 & 0.505 \\
\hline $13.75-12.50$ & 9 & 31.52 & 0.269 & 31.80 \\
\hline $12.50-11.00$ & 8 & 19.15 & 0.137 & 19.29 \\
\hline $11.00-8.00$ & 7 & 3.71 & 0.047 & 3.76 \\
\hline $8.00-4.00$ & 6 & - & 0.01 & 0.01 \\
\hline
\end{tabular}


This equation may be used to find the resonance capture integral for resolved resonances, when $\Gamma_{n}, \Gamma_{\gamma}, \Gamma$, and $E_{O}$ are available, as in references (3) and (4).

Table 1.2-2 shows the results of calculations of resonance integrals using equation (1.2-43) for resolved resonances in $\mathrm{Th}-232$.

For groups of low neutron energy, the $1 / \mathrm{v}$ contribution to the crosssection must be added. Assuming that the cross-section is given by

$$
\sigma c(v)=\frac{\sigma p^{v} p}{v}
$$

where subscript $\mathrm{p}$ refers to conditions at most probable thermal speed, it may be shown that

$$
\int_{E_{1}}^{E_{2}} \sigma_{c}(E) \frac{d E}{E}=2\left[\sigma_{c}\left(E_{1}\right)-\sigma_{c}\left(E_{2}\right)\right]
$$

The thermal group average is taken by the familiar formula

$$
\bar{\sigma}_{a}=\frac{\sigma_{a p}}{1.128}
$$

For Th-232, a non-1/v correction is not needed. The thermal group average with a cutoff at energy $0.1956 \mathrm{ev}$ has been found different from the above by only about $1 / 4$ percent, which means that a negligible error is incurred with the above formula. Table 1.2-3 shows the contributions to the average group cross-sections for Th-232 from the resolved resonances and the $1 / \mathrm{v}$ absorption, along with the sum of the two.

\subsubsection{Averaging Over Unxesolved Resonances}

The cross-sections for high energy rcsonances have not been determined experimentally because of limitations of present velocity selectors. It is conventional to attempt an extrapolation based on known low energy resonances, following a general appraach suggested ori The theoretical basis for the method is presented below. For a single resonance, the cross-section for the reaction, $(n, x)$ where $x$ is $\gamma$ for capture and $f$ for fission is: 


$$
\sigma(n, x)=\frac{\sigma_{0}}{\left(E-E_{0}\right)^{2}+(\Gamma / 2)^{2}} \frac{\Gamma_{x}}{\Gamma}
$$

where the peak cross-section

$$
\sigma_{0}=4 \pi \chi^{2} g \frac{\Gamma n}{\Gamma}
$$

Assume that in a region of energy $\Delta \mathrm{E}$ there are $\mathrm{N}$ resonances, equally spaced with level spacing $D$, as shown in Figure 1.2-1.

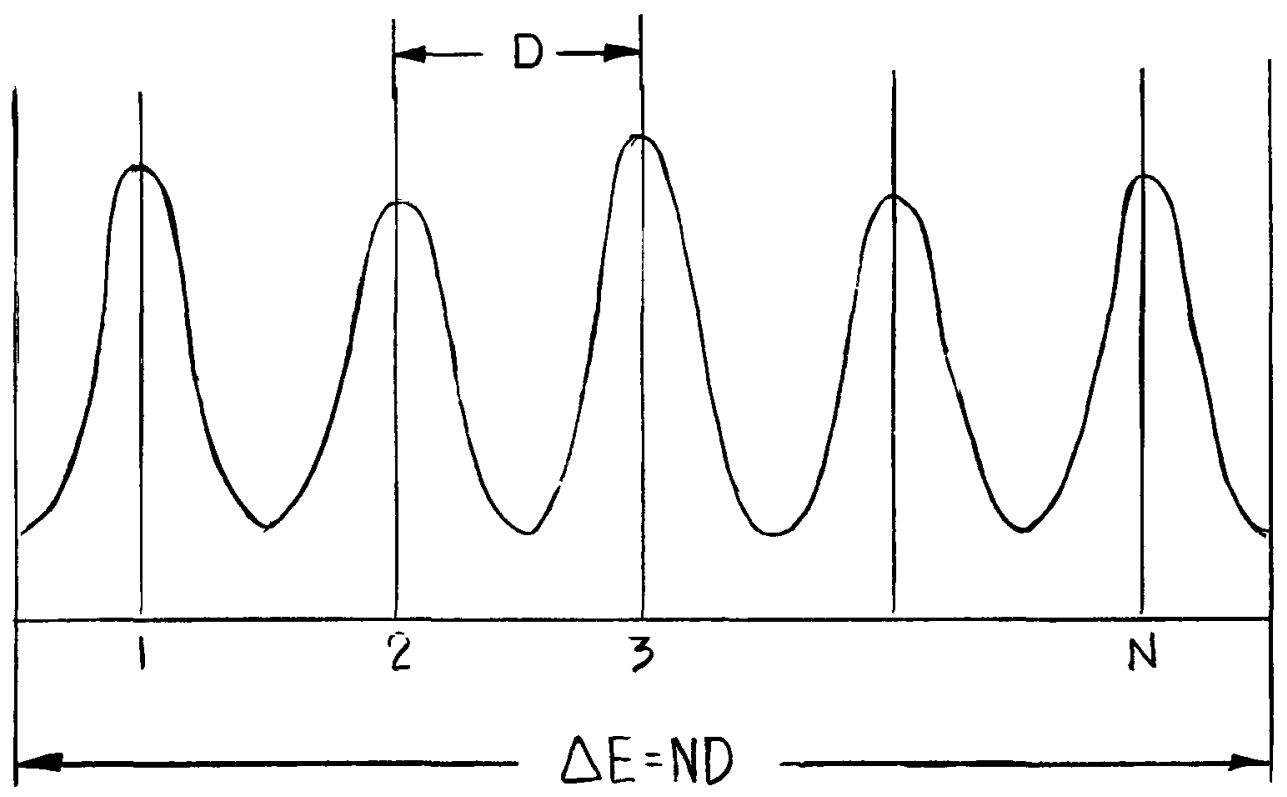

Figure 1.2-1 Method of Averaging Cross-sections Over Several Resonances 
Define the average cross-section as:

$$
\sigma(n, x)=\frac{\sum_{i=1}^{N} \int \sigma_{i}(n, x) d E}{\Delta E}
$$

If all the resonances within a region were identical in character, this could be written,

$$
\sigma(n, x)=\frac{N \int \sigma(n, x) d E}{\Delta E}
$$

or since $N D=\Delta E$

$$
\bar{\sigma}(n, x)=\frac{1}{D} \int \sigma(n, x) d E
$$

The resonance parameters such as $\Gamma_{\mathrm{n}}, \Gamma_{\mathrm{f}}, \Gamma_{\gamma}$, actually will not be identical. As found in the low-energy resonances, there are distributions of these quantities. Thus,

$$
P\left(r_{n}\right) d r_{n}
$$

represents the fraction of the levels that have a neutron width in the range $d \Gamma_{n}$ at $\Gamma_{n}$. The average should be generalized to read

$$
\bar{\sigma}(n, x)=\frac{1}{D} \int d E \iiint P\left(\Gamma_{n}\right) d \Gamma_{n} P\left(\Gamma_{f}\right) P\left(\Gamma_{\gamma}\right) d \Gamma_{f} d \Gamma_{\gamma} \sigma(n, x)(1.2-51)
$$

Consider the case of a non-fissionable element for which $\Gamma_{\gamma}$ is independent of energy (an accurate assumption, as inspection of reference (3) will reveal). Then, the fission distribution function is missing and

$$
\bar{\sigma}(n, \gamma)=\frac{1}{D} \int d E\left(P\left(\Gamma_{n}\right) d \Gamma_{n} \sigma(n, x)\right.
$$

Inserting the Breit-Wigner formula and integrating over energy,

$$
\bar{\sigma}(n, y)=\frac{2 \pi^{2} x^{2} g}{D} \int \frac{\Gamma_{n} \Gamma x p\left(\Gamma_{n}\right) d \Gamma_{n}}{\Gamma}
$$


There is much controversy over the proper choice of distribution function in $\Gamma$ n. Evidence based on studies by Hughes (ㅁ) and Harvey (6) of many elements points to the exponential expression:

$$
P\left(\Gamma_{n}\right) d \Gamma_{n}=e^{-x} d x
$$

where

$$
x=\frac{\Gamma_{n}}{\bar{\Gamma}_{n}}
$$

Then integrating equation $(1.2-53)$ yields

$$
\bar{\sigma}(n, \gamma)=\frac{4.09 \times 10^{6} g \Gamma \gamma}{D E}\left[1-y e^{y} E_{1}(y)\right]
$$

where

$$
y=\frac{\Gamma x}{\bar{\Gamma} n}
$$

and $E_{1}(y)$ is the exponential integral.

Some investigations make use of theoretical estimates of the level spacing $D$ based on the Weisskopf (ㄱ) formula

$$
D=\frac{e^{-2 \sqrt{a \varepsilon}}}{c}
$$

where a and $c$ are parameters that vary slowly with atomic weight. The effect of higher angular momentum neutrons is also applied, by multiplying by a factor

$$
\frac{L^{*}}{D}=(1+k R)^{2}
$$

involving the wave number and nuclear radius. In view of Weisskopf's own admission that equation $(1.2-56)$ formula should not be used quantitatively, it was regarded as preferable to use the available crosssection data as a source of information on $D$ trends, rather than vice versa. The procedure adopted was therefore as follows: 
(a) To plot graphs of the level spacing at low neutron energy, from the difference of tabulated resonance energies.

(b) To compute the theoretical cross-section (determined except for D/I) from equation (1.2-55) at a number of points in the high energy region, where experimental data averaged over resonances is available.

(c) To deduce D/I as a function of energy and plot on the same graph as for low levels.

(d) To interpolate the $\mathrm{D} / \mathrm{I}$ graph over the relatively small region of energy in which no data are given in references (3) and (4).

(e) To calculate the average cross-section in the above region using appropriate level spacings.

It is to be noted that the above method takes maximum advantage of experimental data. Table 1.2-4 gives the average group capture cross-sections in the high energy and intermediate energy regions for $\mathrm{Th}-232$.

Table 1.2-5 combines the results of the previous tables to give the final group average capture cross-sections for $\mathrm{Th}-232$.

\subsubsection{Fission Cross-sections}

The average cross-sections for fission in the high energy region are formed from BNL values or values obtained by deduction or extrapolation. Table 1.2-6 lists the average fission cross-sections for Th-232.

$\cdot$

\subsubsection{Theory of Average Cross-sections for Fissionable Materials}

The general formula for the cross-section for a process $\sigma(n, x)$, where $x$ may be capture $(\gamma)$ or fission ( $f), \sigma$ averaged over several resonances near an energy $E$ is given by equation (1.2-51). The local cross-section is given by the usual Breit-Wigner formula, equation (1.2-46). In most fertile or fissionable materials, the gamma width $\Gamma \gamma$ is approximately constant. Hence the use of a distribution function in that variable is not needed. With $\Gamma_{\gamma}$ independent of energy, by 
TABLE 1.2-4

INTERMEDIATE AND HIGH ENERGY CONTRI BUTIONS TO GROUP

CROSS-SECTIONS AND RESONANCE INTEGRALS FOR Th-232

$\begin{array}{ccc}\text { Group Number } & \frac{\bar{\sigma}_{c}}{7} & \frac{\sigma_{r}}{2.47} \\ 6 & 0.63 & 7.41 \\ 5 & 0.22 & 2.52 \\ 4 & 0.130 & 0.22 \\ 3 & 0.050 & 0.13 \\ 2 & 0.013 & 0.05 \\ 1 & 0.004 & 0.01 \\ & & \text { neg. }\end{array}$


TABLE $1.2-5$

FINAL COMPILATION, GROUP CAPTURE

CROSS-SECTIONS FOR Th-232

Group Number

Thermal

13

12

11

10

9

8

7

6

5

4

3

2

1
$T h-232$

$\sigma_{c}$

6.605

2. 235

1. 448

0.880

0.505

31.80

19.29

6.23

0.64

0.22

0.13

0.05

0.013

0.004 
TABLE $1.2-6$

FISSION CROSS-SECTIONS FOR Th-232

Group Number

4

3

2

1 $\left(\sigma_{f}\right) 232$ barns

0.002

0.12

0.21

0.40

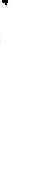


inserting the Breit-Wigner formula in equation (1.2-51) and integrating, one obtains for the capture cross-section

$$
\left.\bar{\sigma}(n, \gamma)=\frac{2 \pi^{2} \lambda^{2} g}{D} \iint \frac{\Gamma_{n} \Gamma_{\gamma}}{\Gamma} P\left(\Gamma_{n}\right) d \Gamma_{n} P\left(\Gamma_{\gamma}\right) d\right) d \Gamma_{\gamma}
$$

and for the average fission cross-section

$$
\bar{\sigma}(n, f)=\frac{2 \pi^{2} x^{2} g}{D} \int \frac{\Gamma_{n} \Gamma_{f}}{\Gamma} P\left(\Gamma_{n}\right) d \Gamma_{n} P\left(\Gamma_{f}\right) d \Gamma_{f}
$$

If the distribution functions can be specified, then the integration may be performed.

An examination of the resolved resonances in the low energy region, less than $100 \mathrm{ev}$, reveals that the neutron width is proportional to the square root of the energy, by a factor of $\Gamma_{n}^{\circ}$, the reduced neutron width, defined by

$$
\Gamma_{n}^{0}=\Gamma_{n}\left(\frac{\epsilon}{E}\right)^{\frac{1}{2}}
$$

where $\varepsilon$ is chosen as $1 \mathrm{ev}$. It is also found that the level spacing $D$ is roughly constant. The best distribution function for neutron widths is considered to be the exponential function

$$
P\left(\Gamma_{n}\right) d \Gamma_{n}=e^{-x} d x
$$

The best distribution for the $\Gamma_{f}$ for fission is believed to be of the form

$$
P\left(\Gamma_{f}\right) \cdot d \Gamma_{f}=y^{\prime} e^{-y^{\prime}} d y^{\prime}
$$

where

$$
y^{\prime}=\frac{2 \Gamma_{f}}{\Gamma_{f}}
$$


This distribution function is normalized to unit area $P\left(\Gamma_{f}\right) d \Gamma_{f}=1$; the average fission width is $\Gamma_{f}=\int \Gamma_{f} P\left(\Gamma_{f}\right) d \Gamma_{f}$.

In the case of U-233, with limited data, the application of the general formula is needed. Equations $(1.2-54)$ and $(1.2-61)$ are inserted in equations $(1.2-58)$ and $(1.2-59)$ and the integration performed. The $I$ integrals appearing in the formulas below are listed in section (2.2.7)

$$
\begin{aligned}
& \bar{\sigma}(n, \gamma)=\frac{8 \pi^{2} \not^{2} g \Gamma x}{D \bar{\Gamma}_{n} \bar{\Gamma}_{f}^{2}} I_{11} \\
& \bar{\sigma}(n, f)=\frac{8 \pi^{2} \chi^{2} g}{D \bar{\Gamma}_{n} \bar{\Gamma}_{f}^{2}} I_{21}
\end{aligned}
$$

\subsubsection{Calculations of U-233 Cross-sections}

The thermal group cross-sections were obtained from the world consistent figures

$$
\begin{aligned}
& \sigma c=56 \text { barns } \\
& \sigma f=532 " \text { " } \\
& \sigma a=588 " ~
\end{aligned}
$$

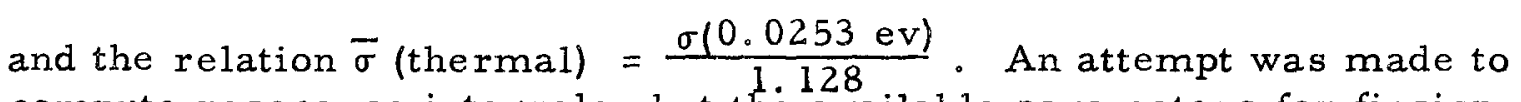
compute resonance integrals, but the available parameters for fission were very ill-defined. For groups 13 and 12, immediately above thermal, numerical integration of the data for both $\sigma_{f}$ and $a=\sigma_{c} / \sigma_{f}$ in reference (4) was possible. For groups 11 and 10, 6, 5, and 4, numerical integration of $\sigma_{f}$ only from reference (4) was performed. For groups 1, 2, ani 3, a constant fission cross-section of 1.9 barns was assumed.

For groups 9, 8 and 7 , the theory of average fission cross-sections as embodied in equation (1.2-63) was employed to determine the variations of $\sigma_{f} \sqrt{E}$ for energies ranging from $10 \mathrm{ev}$ to $10^{4} \mathrm{ev}$. A complete curve was developed for $\sigma_{f}$ through the region in which tabulated data are weak. Numerical integration of the resulting curve was then done. 
After obtaining the above set of fission cross-sections, a calculation was made of the variation $a=\Gamma \gamma I_{1} / I_{21}$, (see equations (1.2-62 and 1.2-63), from low energies up to $10 \%$. It was found necessary to carry as many as 9 significant figures along to guarantee accuracy and consistency of the final integrals for $I_{11}$ and $I_{21}$. Since a was observed to vary slowly $\left(\simeq 0.5\right.$ at low energies to 0.4 at $\left.10^{7} \mathrm{ev}\right)$, the capture crosssections could be computed by multiplying by typical a values over a lethargy range. It should be emphasized that these capture values are rather uncertain, because the best available theory still gives only rough agreement with experiment in the case of $U-235$. The final results are listed below.

TABLE $1.2-7$

Average Group Cross-sections for U - 233

$\begin{array}{crrr}\text { Group } & \sigma_{\mathrm{c}} \text { (barns) } & \sigma_{\mathrm{f}} \text { (barns) } & a=\frac{\sigma_{\mathrm{C}}}{\sigma_{\mathrm{f}}} \\ \text { Thermal } & 49.6 & 472.0 & 0.11 \\ 13 & 23.0 & 173.0 & 0.13 \\ 12 & 17.0 & 132.0 & 0.13 \\ 11 & 172.0 & 325.0 & 0.53 \\ 10 & 63.0 & 121.0 & 0.52 \\ 9 & 33.0 & 63.5 & 0.52 \\ 8 & 18.0 & 35.0 & 0.52 \\ 7 & 5.9 & 11.6 & 0.51 \\ 6 & 2.0 & 4.1 & 0.49 \\ 5 & 1.1 & 2.2 & 0.47 \\ 4 & 0.9 & 2.0 & 0.46 \\ 3 & 0.8 & 1.9 & 0.44 \\ 2 & 0.8 & 1.9 & 0.43 \\ 1 & 0.8 & 1.9 & 0.41\end{array}$


1.2.2.7 Evaluation of Integrals

Oleska's integrals for fissionable materials (8).

$$
\begin{aligned}
& I_{11}=\frac{2}{(b-a)^{3}}\left[e^{b \Gamma_{\gamma}} E_{1}\left(b \Gamma_{\gamma}\right)-e^{a \Gamma_{\gamma}} E_{1}\left(a \Gamma_{\gamma}\right)\right] \\
& +\frac{1}{(b-a)^{2}}\left[-\Gamma_{\gamma} e^{a \Gamma_{\gamma}} E_{1}\left(a \Gamma_{\gamma}\right)+\frac{1}{a}-\Gamma_{\gamma} e^{b \Gamma_{\gamma}} E_{1}\left(b \Gamma_{\gamma}\right)+\frac{1}{b}\right]
\end{aligned}
$$

where

$$
a=\frac{2}{\bar{\Gamma}_{f}}, b=\frac{1}{\bar{\Gamma}_{n}}
$$

$$
\begin{aligned}
I_{21} & =\frac{6}{(a-b)^{4}}\left[e^{a \Gamma_{\gamma}} E_{1}\left(a \Gamma_{\gamma}\right)-e^{b \Gamma_{\gamma}} E_{-1}\left(b \Gamma_{\gamma}\right)\right] \\
& +\frac{4}{(a-b)^{2}}\left[-\Gamma_{\gamma} e^{a \Gamma_{\gamma}} E_{1}\left(a \Gamma_{\gamma}\right)+\frac{1}{a}\right] \\
& +\frac{2}{(a-b)^{3}}\left[-\Gamma_{\gamma} e^{b \Gamma_{\gamma}} E_{1}\left(b \Gamma_{\gamma}\right)+\frac{1}{b}\right] \\
& +\frac{1}{(a-b)^{2}}\left[\Gamma_{\gamma}^{2} e^{a \Gamma_{\gamma}} E_{1}\left(a \Gamma_{\gamma}\right)-\frac{\Gamma_{\gamma}}{a}+\frac{1}{a^{2}}\right]
\end{aligned}
$$

$1-24$ 
Evaluation of integral appearing in $\bar{\sigma}(n, \gamma)$ for non-fissionable isotope using exponential distribution.

$$
I=\int_{0}^{\infty} \frac{\Gamma_{n} \Gamma_{\gamma}}{\Gamma_{n}+\Gamma_{\gamma}} P\left(\Gamma_{n} d \Gamma_{n}=\Gamma_{\gamma} \int_{0}^{\infty} \frac{x P(x) d x}{x+y}=\Gamma_{\gamma} \int_{0}^{\infty} \frac{x e^{-x} d x}{x+y}\right.
$$

where

$$
y=\frac{\Gamma_{y}}{\bar{\Gamma}_{n}}
$$

Let $x+y=u, d x=d u$ and $x=u-y$

$$
\begin{aligned}
I & =\Gamma_{\gamma} \int_{y}^{\infty} \frac{(u-y) e^{-(u-y)} d u}{u}=\Gamma_{\gamma} e^{y}\left[\int_{y}^{\infty} e^{-u} d u-y \int_{y}^{\infty} \frac{e^{-u} d u}{u}\right] \\
& =\Gamma_{\gamma} e^{y}\left\{\left[-e^{-u}\right]_{y}^{\infty}-y E_{1}(y)\right\} \\
I & =\Gamma_{\gamma}\left[1-y e^{y} E_{1}(y)\right]
\end{aligned}
$$


1.2.2.8 Fast Cross-Section and Group Constants

TABLE $1.2-8$

FAST CROSS-SECTION AND GROUP CONSTANTS

EIGHTEEN GROUP CROSS SECTIONS

\begin{tabular}{|c|c|c|c|c|c|c|c|c|}
\hline $\begin{array}{l}\text { Group } \\
\text { No. }\end{array}$ & $\begin{array}{l}\text { Lethargy } \\
\text { Range }\end{array}$ & $\begin{array}{c}\overline{\sigma_{c}} \\
(T h-232) \\
\end{array}$ & $\begin{array}{c}\overline{\sigma_{c}} \\
(U-233)\end{array}$ & $\begin{array}{c}\overline{\bar{T}_{r}} \\
(U-233)\end{array}$ & $\begin{array}{c}\bar{\sigma}_{5} \\
(\mathrm{U}-233) \\
\end{array}$ & $\begin{array}{c}\bar{\sigma}_{s} \\
(\mathrm{Th}-232) \\
\end{array}$ & $\begin{array}{l}\bar{\sigma}_{s} \\
\text { (c) }\end{array}$ & $\begin{array}{l}\bar{\pi} \\
(0) \\
\end{array}$ \\
\hline 1 & 0.0 .5 & 0.004 & 0.8 & 1.9 & 1.75 & - & 0.64 & 1.75 \\
\hline 2 & $0.5-1$ & 0.013 & 0.8 & 1.9 & 2.40 & - & 0.74 & 1.15 \\
\hline 3 & $1-2$ & 0.050 & 0.8 & 1.9 & 3.60 & 6.99 & 1.39 & 2.06 \\
\hline 4 & $2-3$ & 0.130 & 0.9 & 2.0 & 5.40 & 6.81 & 2.66 & 4.20 \\
\hline 5 & $3-4$ & 0.22 & 1.1 & 2.2 & 7.50 & 9.11 & 2.76 & 4.56 \\
\hline 6 & $4-5$ & 0.32 & 1.2 & 2.52 & 9.1 & 10.5 & 4.59 & 3.52 \\
\hline 7 & $5-6$ & 0.47 & 1.43 & 2.94 & 10.4 & 11.5 & 4.7 & 3.50 \\
\hline 8 & $6-7$ & 0.70 & 2.02 & 4.09 & 11.5 & 11.5 & 4.7 & 3.70 \\
\hline 9 & $7-8$ & 1.08 & 3.25 & 6.51 & 12.5 & 11.12 & 4.7 & 3.80 \\
\hline 10 & $8-9$ & 1.75 & 3.28 & 6.50 & 13.3 & 10.63 & 4.7 & 3.80 \\
\hline 11 & $9-10$ & 2.49 & 5.92 & 11.6 & 13.8 & $1 d .4$ & 4.7 & 3.80 \\
\hline 12 & $10-11$ & 14.44 & 9.53 & 18.5 & 14.0 & 10.4 & 4.7 & 3.80 \\
\hline 13 & $11-12$ & 24.28 & 16.06 & 31.0 & 14.1 & 10.7 & 4.7 & 3.80 \\
\hline 14 & $12-13$ & 28.32 & 24.2 & 46.5 & 14.1 & 10.12 & 4.7 & 3.80 \\
\hline 15 & $13-14$ & 16.16 & 59.38 & 114.2 & 13.8 & 11.4 & 4.7 & 3.80 \\
\hline 16 & $14-15$ & 0.53 & 48.0 & 92.28 & 13.7 & 11.3 & 4.7 & 3.80 \\
\hline 17 & $15-16$ & 0.88 & 172.0 & 325.0 & 13.3 & 11.6 & 4.7 & 3.80 \\
\hline 18 & $16-17$ & 1.45 & 17.0 & 132.0 & 12.8 & 11.8 & 4.7 & 3.80 \\
\hline
\end{tabular}


TABLE $1.2-9$

EIGHTEEN GROUP CONSTANTS

\begin{tabular}{|c|c|c|c|c|c|}
\hline Group No. & $X(\mathrm{U}-233) *$ & $\bar{\mu}(0)$ & $\xi \quad 0$ & $\bar{\mu}_{\text {(C) }}$ & $\xi 10$ \\
\hline 1 & 0.02027 & 0.688 & 0.038 & 0.610 & 0.056 \\
\hline 2 & 0.10324 & 0.582 & 0.051 & 0.458 & 0.088 \\
\hline 3 & 0.45353 & 0.340 & 0.079 & 0.249 & 0.124 \\
\hline 4 & 0.30201 & 0.170 & 0.1035 & 0.151 & 0.143 \\
\hline 5 & 0.09233 & 0.095 & 0.1122 & 0.105 & 0.150 \\
\hline 6 & 0.02236 & 0.039 & 0.1175 & 0.017 & 0.155 \\
\hline 7 & 0.00548 & 0.001 & 0.1198 & - & 0.158 \\
\hline 8 & 0.00074 & - & 0.12 & - & 0.158 \\
\hline 9 & - & - & 0.12 & - & 0.158 \\
\hline 10 & - & - & 0.12 & - & 0.158 \\
\hline 11 & - & - & 0.12 & - & 0.158 \\
\hline 12 & - & - & 0.12 & - & 0.158 \\
\hline 13 & - & - & 0.12 & - & 0.158 \\
\hline 14 & - & - & 0.12 & - & 0.158 \\
\hline 15 & - & - & 0.12 & - & 0.158 \\
\hline 16 & - & - & 0.12 & - & 0.158 \\
\hline 17 & - & - & 0.12 & - & 0.158 \\
\hline 18 & - & - & 0.12 & - & 0.158 \\
\hline
\end{tabular}

* Normalized to one neutron per fission. 


\subsubsection{Flux Depression and Doppler Effect (9)}

In the preceding section, group constants for fertile and fissionable elements based in part on the resonance integral were prepared, These values, which were computed by a combination of theory and available data, represent the effective cross-sections in the idealized situation of very dilute systems such that the Doppler effect is negligible. This section covers the modification of the above data to include higher concentrations of fuel and fertile material, and the specific Doppler broadening corrections. The fuel and moderator are presumed to be homogeneously mixed, which eliminates the need for geometric self-shielding. The self-shielding associated with flux depressions in energy will however be retained. The moderator will be considered to be a relatively heavy element, into which category graphite reasonably falls.

Convenient graphs for the computation of self-shielding factors are presented for use with a 59 group division, for $\mathrm{Th}-232$ and $\mathrm{U}$ 233 in graphite at a variety of moderator-absorber ratios and temperatures in the range from room temperature to $1600^{\circ} \mathrm{F}$.

\subsubsection{Theory and Development of Equations}

Short of application of the rigorous transport solution of the neutron slowing problem under conditions of strong absorption, the method attributed to Goertzel and Greuling $(G-G)(10)$ is considered the most accurate available. It is summarized below.

The basic equations for slowing in a heavy element are the collision density.

$\phi(u) \Gamma .(u)=S(u)+\int_{u+l n \alpha}^{u} \phi(u) \sum s\left(u^{\prime}\right) \frac{e^{-\left(u-u^{\prime}\right)}}{1-\alpha} d u^{\prime}(1.2-64)$

and the slowing down density

$$
q(u)=\int_{u+\ln \alpha}^{u} \phi\left(u^{\prime}\right) \sum_{s}\left(u^{\prime}\right) \frac{\left[e^{-\left(u-u^{\prime}\right)}\right]}{1-\alpha} d u^{\prime}
$$


where $\alpha=\left(\frac{A-1}{A+1}\right)^{2}$

$S(u)$ is the fission source, and the fluxes and cross-sections have conventional meaning. The essence of the G-G method is that the scattering collision density $\psi_{\mathrm{s}}=\phi_{\mathrm{s}}$ may be represented by a two-
term expansion

$$
\psi_{s}\left(u^{\prime}\right) \simeq \psi_{s}(u)+\left[\frac{d \psi_{s}\left(u^{\prime}\right)}{d u^{\prime}}\right]_{u}\left(u^{\prime}-u\right)
$$

which is valid if $\phi(u)$ is relatively slowly varying with lethargy.

Solving equations $(1.2-64),(1.2-65)$, and $(1.2-67)$, the G-G resonance escape probability becomes

$$
P=e^{-\int_{0}^{u} \frac{\sum_{a}\left(u^{\prime}\right) d u^{\prime}}{\xi \sum_{s}\left(u^{\prime}\right)+\gamma \sum_{a}\left(u^{\prime}\right)}}
$$

where

$$
\xi^{\prime}=\frac{2}{A+2 / 3}
$$

and

$$
\gamma=1-\frac{\alpha}{2 \xi^{\prime} \alpha}\left(1-\xi^{\prime}\right)^{2}
$$

The Doppler effect is incorporated in the resonance absorption and scattering cross-sections by the use of the tabulated (11) function $\Psi(x, \xi) \quad$ Thus

$$
\sigma(x)=\sigma_{0} \Psi(x, \xi) \text { total cross section }(1.2-71)
$$




$$
\begin{aligned}
& \sigma_{a}(x)=\sigma(x) \frac{\Gamma_{\gamma}}{\Gamma} \quad \begin{array}{l}
\text { absorption cross } \\
\text { section }
\end{array} \\
& \sigma_{s}(x)=\sigma(x) \frac{\Gamma_{n}}{\Gamma} \quad \begin{array}{l}
\text { scattering cross } \\
\text { section }
\end{array} \quad(1.2-73) \\
& \text { where } \left.X=\frac{E-E_{0}}{(\Gamma / 2)}\right\} \quad \text { reduced variables }(1.2-74) \\
& \xi=\Gamma / \Delta\} \\
& \Delta=\left(\frac{4 m k T E_{0}}{M}\right)^{\frac{1}{2}} \quad \text { Doppler width } \quad(1.2-75) \\
& \sigma_{0}=\frac{2.6 \times 10^{6} \Gamma_{n} g}{E_{0} \Gamma} \quad \begin{array}{l}
\text { peak total } \\
\text { cross section }
\end{array} \quad(1.2-76)
\end{aligned}
$$

A distinction is made between the resonance scattering crosssection $\sigma_{s}$ and the potential scattering cross-section $\sigma_{p}$ which includes the constant moderator and fuel scattering cross-sections in the vicinity of a resonance. Thus one writes for a mixture of fuel (0) and moderator (1)

$$
\Sigma_{s}=N_{0} \sigma_{P O}+N_{1} \sigma_{S 1}+N_{0} \sigma_{s o}
$$

where the terms, in order, are fuel potential, moderator and fuel resonance scattering. Combining as follows,

$$
\sum_{p}=N_{0} \sigma_{p c}+N_{1} \sigma_{s 1}=N_{0} \sigma_{p}
$$

and inserting equations $(1.2-78),(1.2-72)$ and $(1.2-73)$ in equation $(1.2-67)$

$$
P=e^{\frac{-N_{0} \sigma_{r e}}{\xi \sum p}}
$$


where the effective resonance integral is given by

$$
\sigma_{r e}=\int_{-\infty}^{\infty} \frac{\sigma_{0} \frac{\Gamma_{x}}{\Gamma} \psi \frac{d E}{E}}{1+\frac{\sigma_{0}}{\sigma_{p}}\left(\Gamma_{n}+\frac{\gamma}{\xi} \Gamma_{\gamma}\right) \frac{\psi}{\Gamma}}
$$

Changes of variable are made in order to express equation (1.2-80) in terms of Dresner's J functions (12).

$$
\sigma_{r e}=\frac{\sigma_{p}^{\prime} \Gamma_{\gamma}}{E_{0}} \int \frac{\psi(x \xi) d x}{\beta^{\prime}+\psi(x, \xi)}=\frac{\sigma_{p}^{\prime} \Gamma_{\gamma}}{E_{0}} J\left(\xi, \beta^{\prime}\right)
$$

where

$$
\sigma_{p}^{\prime}=\frac{\sigma_{p} T}{\Gamma_{n}+\frac{\gamma}{\xi} \Gamma_{\gamma}}
$$

and $\beta^{\prime}=\sigma_{p}^{\prime} / \sigma_{0}$

The effective resonance integral is to be compared with the infinite dilution result from which previous calculations were made

$$
\left(\sigma_{r e}\right)_{\infty}=\frac{\pi}{2} \frac{\Gamma_{y} \sigma_{0}}{E_{0}}
$$

The ratio of the two functions given by equations $(1.2-81)$ and (1.2-84) may be labled a "self-shielding factor",

$$
f=\frac{\sigma_{r e}}{\left(\sigma_{r e}\right)}=\frac{\pi}{2} \beta^{\prime} T\left(\xi, \beta^{\prime}\right)
$$

This number is always less or equal to unity, by virtue of the fact that the denominator, $(\sigma \mathrm{re})_{\infty}$, is obtained by letting $\sigma_{\mathrm{p}}$ go to infinity in equation $(1.2-80)$. 
1.23 .2 Preparation of Graphs and Tables for Calculation of $\mathrm{f}$.

The discussion below refers principally to the treatment of Th-232 for which resonance data is fairly good.

The tabular data of Dresner on the function $J(\xi, \beta)$ were employed. Equation (1.2-85) was plotted as a function of $k$,

where

$$
\beta=10^{-5} ?^{k}
$$

(with $\beta$ replaced by $\beta /$ ) to form graphs of $f$ as a function of $k$. These are shown in Figure 1.2-2. Figure 1.2-3 gives a convenient working plot of $\mathrm{k}$ as a function of $\beta$, based on equation (1.2-86). It was necessary to perform a number of additional numerical integrations of the function $\int_{0}^{\infty} \frac{\psi(\xi, x) d x}{\psi(\xi, x)+\beta}=J(\xi, \beta)$ to extend the tables to the case $\xi=0.05$ for several values of $k$.

The individual resonance data for $T h-232$ were revaluated to provide $\sigma_{0}, \mathbf{r}$ and the function

$$
\frac{\beta^{\prime}}{\sigma_{p}}=\frac{\Gamma}{\sigma_{0}\left(\Gamma_{n}+\frac{\gamma}{\xi} \Gamma_{\gamma}\right)}
$$

for each resonance, using a graphite moderator, which fixes $y$ and $\xi$. These are shown in Table 1.2-10 along with the infinite resonance integral $\left(\sigma_{\mathrm{re}}\right)_{\infty}$.

Charts of the function $\xi$ appearing in equation (1.2-85) for a chosen set of temperature values and for the known resonances were prepared. These are given in Table 1.2-11. 
GRAPH OF THE SELF-SHIELDING FACTOR

$f$ AGAINST $k$ FOR THE RANGE $0.05 \leqslant \xi \leqslant 1.0$

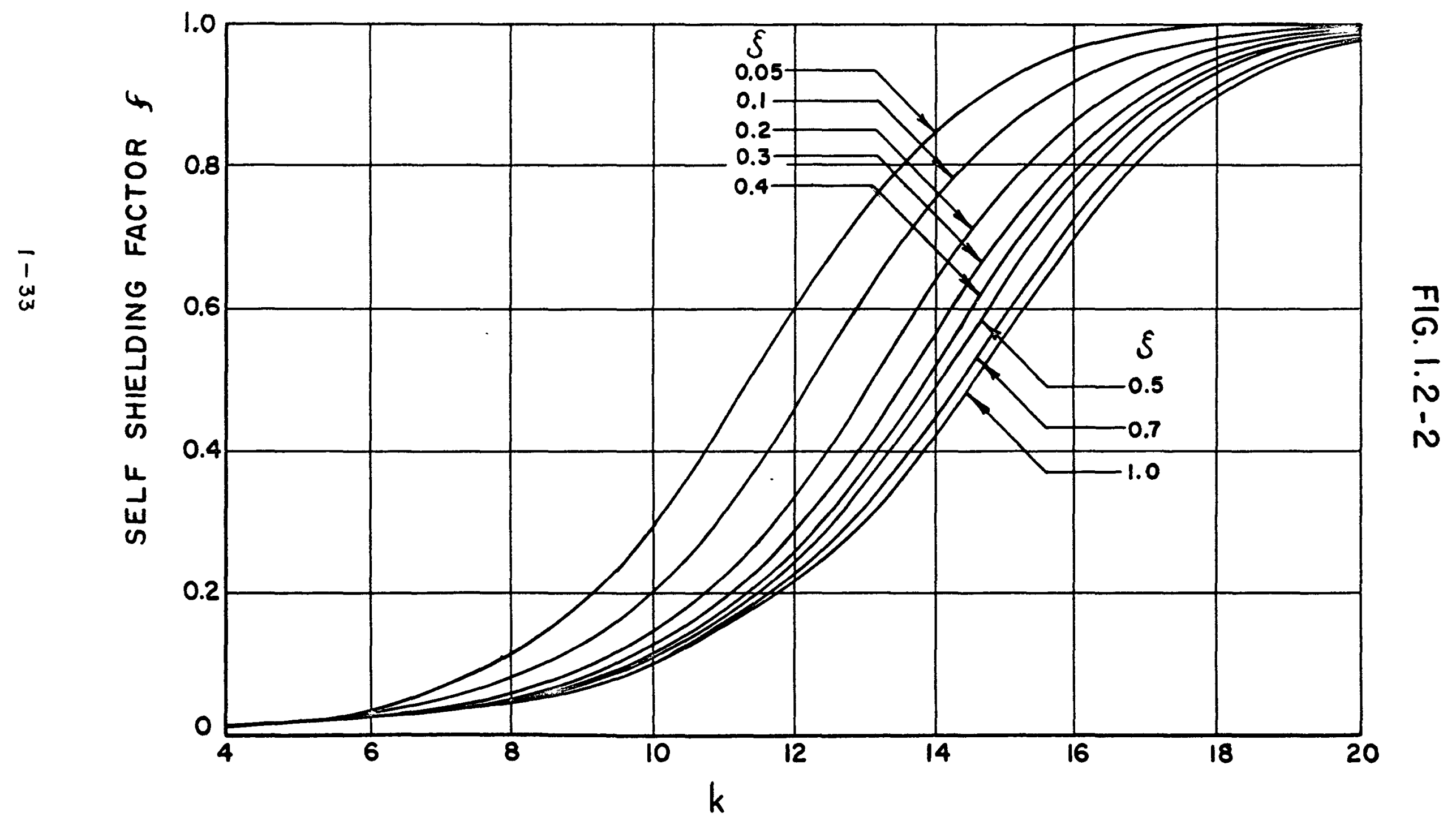


FIG.I.2-3 RELATION OF $K$ AND $\beta^{\prime}$

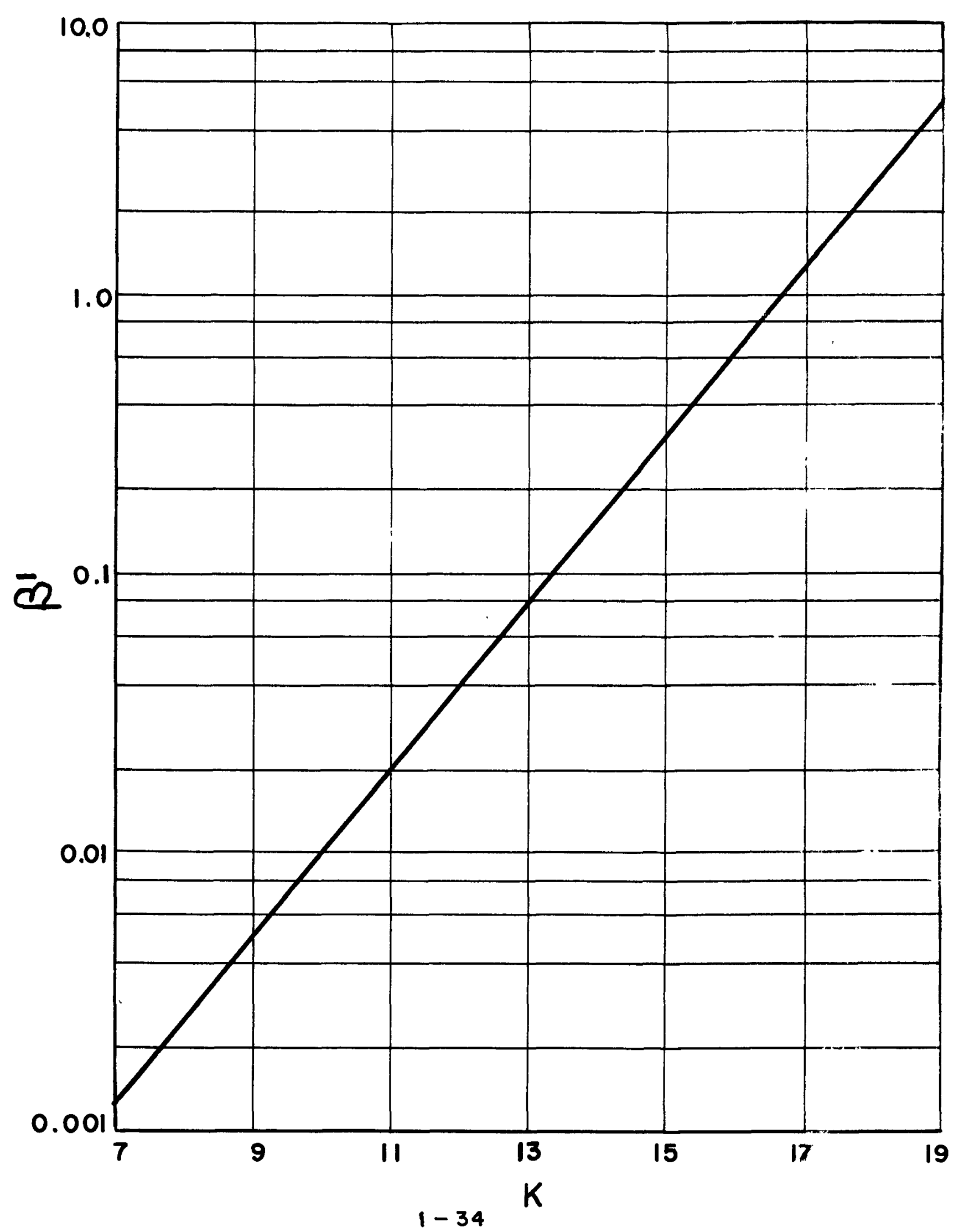


TABLE $1.2-10$

RESOLVED RESONANCE DATA

Th-232

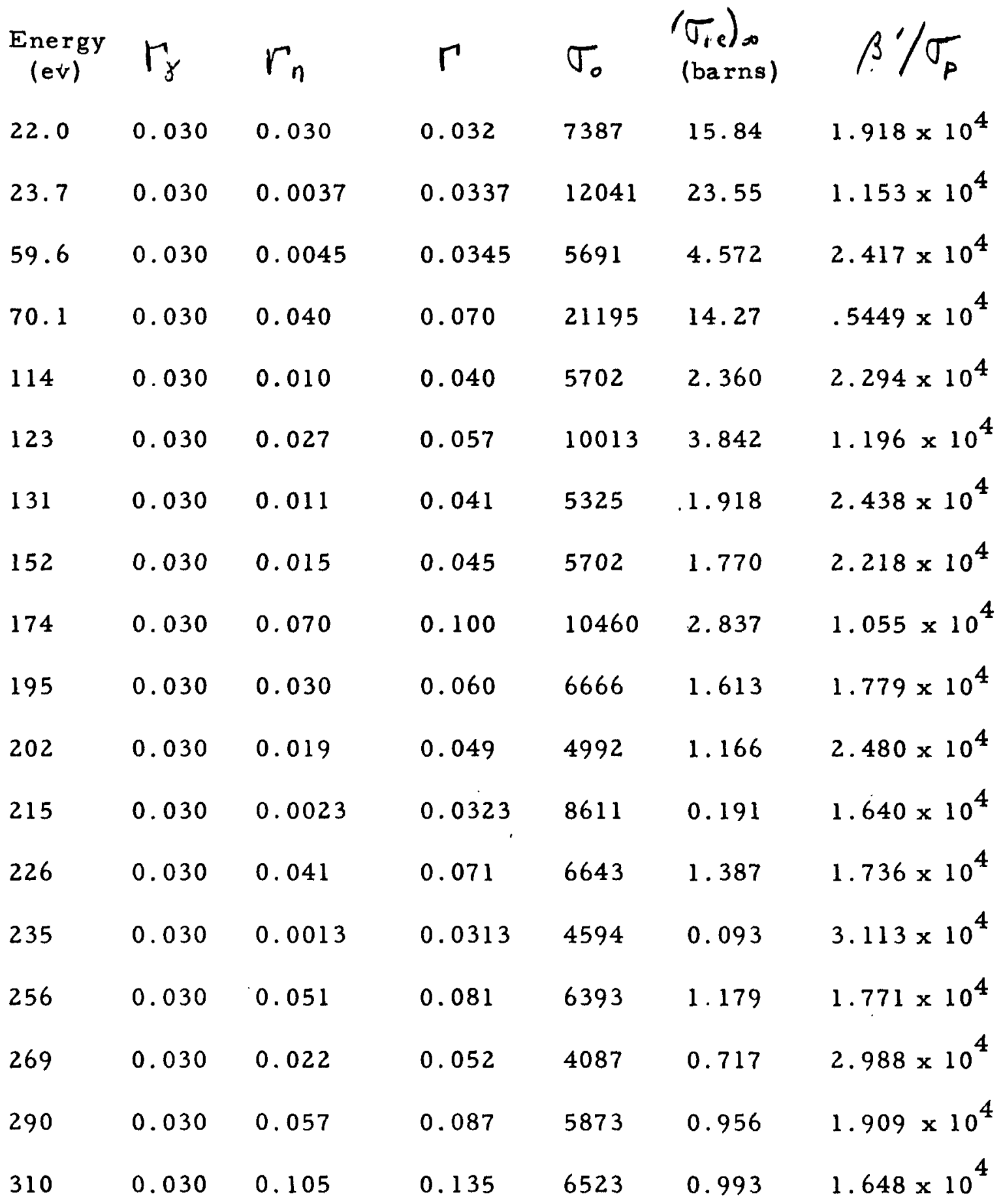


Table 1.2-11

Values of $\xi$ v.s. Temperature for Th-232 Resonances

Temp. in ${ }^{\circ} \mathrm{F}$

\begin{tabular}{|c|c|c|c|c|c|c|c|c|c|}
\hline$E_{O}(e v)$ & $77^{\circ}$ & $212^{\circ}$ & $400^{\circ}$ & $600^{\circ}$ & $800^{\circ}$ & $1000^{\circ}$ & $1200^{\circ}$ & $1400^{\circ}$ & $1600^{\circ}$ \\
\hline 22.0 & .3256 & .2908 & .2573 & .2316 & .2124 & .1974 & .1851 & .1749 & .1662 \\
\hline 23.7 & .3332 & .2976 & .2634 & .2370 & .2174 & .2020 & .1894 & .1790 & .1701 \\
\hline 59.6 & .2100 & .1876 & .1660 & .1494 & .1371 & .1273 & .1194 & .1128 & .1072 \\
\hline 70.1 & .3991 & .3565 & .3154 & .2839 & .2604 & .2419 & .2269 & .2144 & .2037 \\
\hline 114 & .1790 & .1599 & 1415 & .1274 & .1168 & .1085 & .1018 & .0962 & .0914 \\
\hline 123 & .2454 & .2192 & .1939 & .1746 & .1601 & .1488 & .1395 & .1318 & .1253 \\
\hline 131 & .1709 & .1526 & 1351 & .1216 & .1115 & .1036 & .0972 & .0918 & .0872 \\
\hline 152 & .1742 & .1556 & .1377 & .1240 & .1137 & .1056 & .0991 & .0936 & .0889 \\
\hline 174 & .3619 & .3232 & .2860 & .2574 & .2361 & .2194 & .2057 & .1943 & .1847 \\
\hline 195 & .2053 & .1834 & .1622 & .1460 & 1339 & .1244 & .1167 & .1103 & .1048 \\
\hline 202 & .1647 & .1471 & .1302 & .1172 & .1075 & .0998 & .0936 & .0885 & .0841 \\
\hline 215 & .1041 & 0.929 & .0823 & .0740 & .0679 & .0631 & .0592 & .05589 & .0531 \\
\hline 226 & .2253 & .2013 & .1781 & .1613 & .1470 & .1366 & .1281 & .1210 & .1150 \\
\hline 235 & .0964 & .0861 & .0762 & .0686 & .0629 & .0585 & .0548 & .0518 & .0492 \\
\hline 256 & .2416 & .2158 & .1909 & .1718 & .1576 & .1464 & .1373 & .1297 & .1233 \\
\hline 269 & .1513 & .1352 & .1196 & .1076 & .0987 & .0917 & .0860 & .0813 & .07725 \\
\hline 290 & .2401 & .2145 & .1898 & .1708 & .1567 & .1456 &, 1365 & .1290 & .1226 \\
\hline 310 & .3662 & .3270 & .2894 & .2605 & .2389 & .2220 & .2082 & .1966 & .1869 \\
\hline
\end{tabular}


1.2.3.3 Calculational Procedure for Resolved Resonances and 1/v Cross-section

The following procedure was used to compute the self-shielding factor for a given resonance:

1. $\sigma_{p}$ was determined from equation (1.2-78)

2. For the resonance considered, $\beta^{\prime} / \sigma_{p}$ was determined from Table 1.2-10.

3. From 1 . and $2, \beta^{\prime}$ was found and then $K$ was determined from Fig.(1.2-3).

4. The value of $\xi$ was taken from Table 1.2-11.

5. The appropriate self-shielding factor $f$ was then determined from Fig. (1.2-2).

6. The absorption cross section is then calculated by

$$
\bar{\sigma}_{n}=f \frac{\left(\sigma_{r e}\right)_{\infty}}{\Delta u}
$$

The above method takes care of the contribution to the absorption cross-section for a group in which the particular resonance falls. To this must be added the $1 / v$ contribution at this lethargy, which will not be temperature dependent.

The $1 / v$ resonance integral for a group bounded by $u_{1}, u_{2}$ is

$$
\left(\sigma_{r}\right)_{1 / v}=2\left[\sigma_{\alpha}\left(u_{1}\right)-\sigma_{\alpha}\left(u_{2}\right)\right]
$$

the $1 / \mathrm{v}$ law may be expressed in various ways,

$$
\sigma_{a}=\frac{\sigma_{u p} V_{\mu}}{V}=\sigma_{\alpha p} \sqrt{\frac{E p}{E}}=\sigma_{a p} e^{-(u p-u) / 2}(1.2-89)
$$

with $\sigma_{a p}$ the most probable room temperature cross section at speed $V_{p}$ (energy $E_{p}$, lethargy $U_{p}$ ). Combining and going to the average group cross-section due to $1 / \mathrm{v}$

$$
\left(\bar{\sigma}_{a}\right)_{1 / v}=\frac{2 \sigma_{a p}}{u_{1}-u_{2}}\left[e^{-\left(u_{p}-u_{1}\right) / 2}-e^{-\left(u_{p}-u_{2}\right) / 2}\right]
$$

using the 2200 meter cross-section of $\mathrm{Th}-232$ at $\mathrm{U}_{\mathrm{p}}$ of 7.45 barns, and equation (1.2-90), Table 1.2-12 was prepared, whfch covers the groups in which the $1 / v$ contribution is significant. 
TABLE $1.2-12$

CONTRIBUTION TO AVERAGE GROUP CROSS-SECTIONS OF $1 / \mathrm{v}$

$\mathrm{Th}-232$

Lethargy

Group Number

$\sigma_{\alpha}$

17.750

59

2.681

17.511

58

2. 381

17.273

57

17.035

16.811

56

55

54

2. 112

16.588

16.30

53

52

1.879

1.676

1. 489

16.00

51

1. 297

15.753

15.50

15.25

50

1. 125

0.0876

15.00

49

0.8713

14.75

48

0.7682

14.50

47

0.6782

14.25

14.00

13.75

13.50

46

0.5980

45

0.5280

44

0.4660

43

0.4112

13.25

42

0.362

41

0.321

13.00

40

0.283

0.249 


\subsubsection{Unresolved Resonances}

The last resonance in $\mathrm{Th}-232$ for which data are available in reference (11) is at energy $310 \mathrm{ev}$. Beyond that point, the crosssections must be based on combined experimental cross-sections and theoretical interpretation. By the following derivation, it can be shown that previous cross-sections deduced can be corrected for the Doppler effect by simple multiplication by the self-shielding factor $f=\frac{2}{\pi} \beta^{\prime} \tau\left(\xi, \beta^{\prime}\right)$ which can in turn be evaluated as a

function of energy.

The effective resonance integral over an energy range $\Delta E=E_{2}-E_{1}$ that includes identical resonances, $N$ in number, spaced on the average an energy $D$ apart is

$$
\left(\sigma_{r e}\right) \Delta E=\sum_{i-1}^{N}\left(\sigma_{r e}\right)_{i}=\frac{1}{D} \int_{E_{1}}^{E_{2}} d E\left(\sigma_{r e}\right)_{i} \quad(1.2-91)
$$

where $\left(\sigma_{\text {re }}\right) \mathrm{l}$ is the effective resonance integral for the $i^{\text {th }}$ resonance. It is assumed that the distribution of $\Gamma_{n}$ over the range is constant.

Now from equation $(1.2-81)$

$$
\left(\sigma_{r e}\right)_{i}=\frac{\sigma_{p}^{\prime} \Gamma_{\gamma i}}{E_{i}} J\left(\xi_{i}, \beta_{i}\right)
$$

Hence

$$
\left(\sigma_{r e}\right) \Delta F=\frac{\sigma_{p}^{\prime} \Gamma}{D} \int_{E_{1}}^{E_{2}}\left(\xi_{1} \beta\right) \frac{d E}{E}
$$

In considering a group of small energy or lethargy width, $\frac{J\left(\xi_{1} \beta\right)}{E^{\prime}}$ may be replaced by its value at the middle of the group (or the average value) and

$$
\left(\sigma_{r e}\right) \Delta E=\frac{\sigma_{\mu}^{\prime} T_{\gamma}^{\prime}}{\partial E} J\left(\xi, \beta^{\prime}\right) \Delta E
$$


Now if the Doppler effect were ignored or were small, this would reduce to

$$
\left(\sigma_{r e}\right) \Delta E_{1 \infty}=\frac{\sigma_{p}^{\prime} \Gamma_{\gamma}}{D E} \frac{\pi}{2 \beta^{\prime}} \Delta E
$$

The ratio of equations $(1.2-93)$ and $(1.2-94)$ is thus

$$
\frac{\left(\sigma_{r e}\right) \Delta E}{\left(\sigma_{r e}\right) \Delta F_{1 \infty}}=\frac{2}{\pi} \beta^{\prime} J\left(\xi_{1} \beta^{\prime}\right) \equiv f
$$

In order to apply this formula, the variation of $\beta^{\prime} / \sigma_{p}$ and $\xi$ with energy in the region beyond the resolved resonances must be known. The following argument was invoked:

1. $\Gamma_{\gamma}$ is constant at $0.030 \mathrm{ev}$ in the low energy range, and hence may be assumed to continue so.

2. $\Gamma_{n}$ varies with energy in general as $\Gamma_{n}^{0} \sqrt{E}$ where $\Gamma_{n}^{0}$ is the reduced neutron width (the neutron width at $1 \mathrm{ev}$ ). The latter was estimated by taking an average over 18 tabulated resonances, to obtain $\bar{\Gamma}_{n}{ }^{0}=0.00208 \mathrm{ev}$.

Thus $\Gamma=\Gamma_{\gamma}+\Gamma_{n} \quad=0.030+0.00208 \sqrt{\mathrm{E}}$. Equations $(1.2-84),(1.2-85)(1.2-86)$ and $(1.2-87)$ were used to compute $\beta^{\prime} / \sigma_{p}$ and $\zeta$

For purposes of correcting previous cross-section data, Figure $1.2-4$ was used, showing $\beta^{\prime} / \sigma_{p}$ as a function of lethargy over the principal range of interest $300 \mathrm{ev}$ to $3.5 \mathrm{mev}$. In Figure 1.2-5 the proper $\xi$ values for several temperatures are plotted as a function of $u$. The graph was then used to form the appropriate $f$ factors for each group in the unresolved region.

\subsubsection{Treatment of $\mathrm{U}-233$}

The method described above is most appropriate for Th-232, where the resonances are reasonably well resolved. The situation in $\mathrm{U}-233$ is different for several reasons. 
FIG. I.2-4 $\beta^{\prime} / \sigma_{P}$ UNRESOLVED RESONANCES

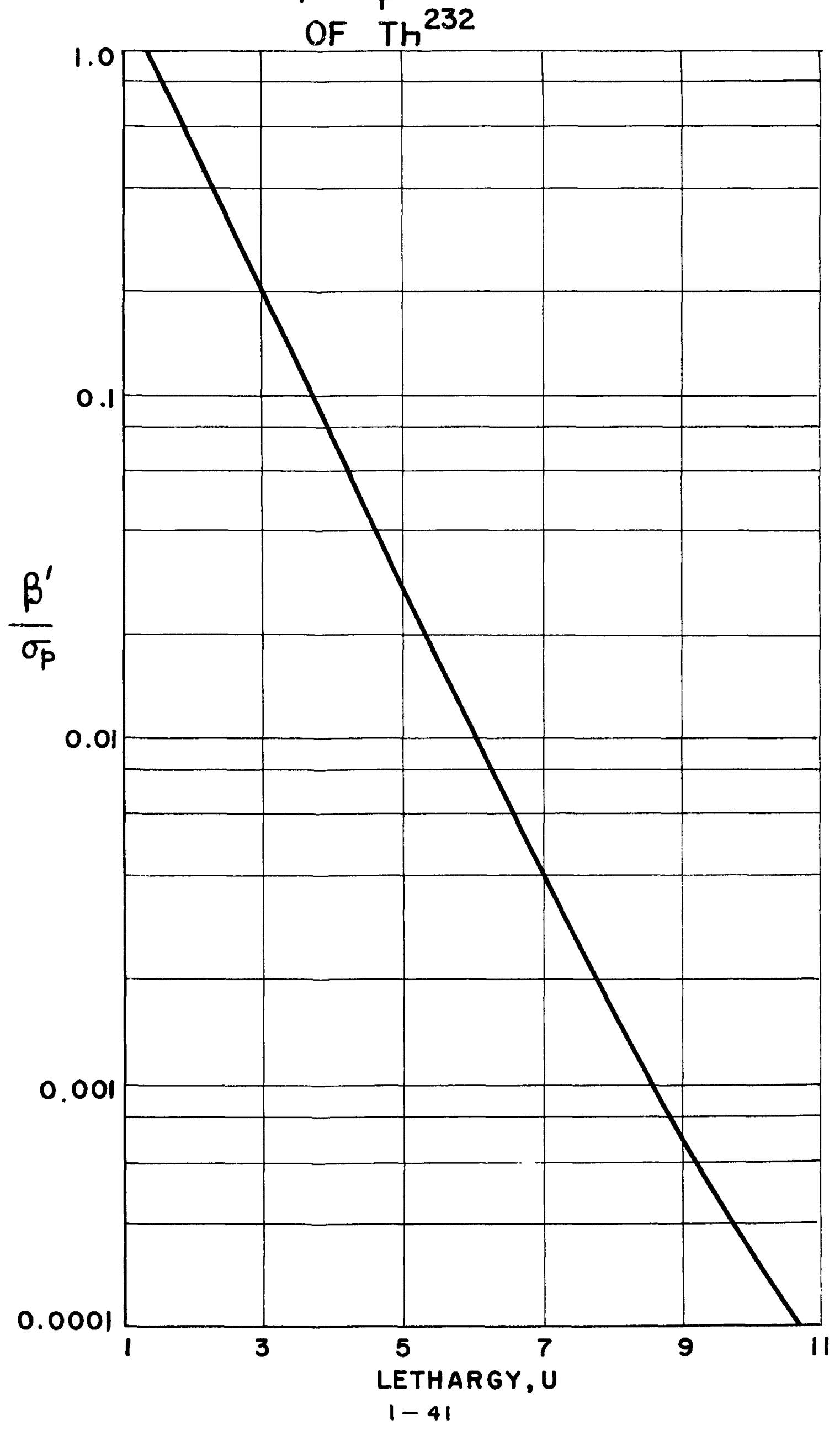


FIG. I.2-5 $\xi(U, T)$ FOR Th ${ }^{232}$ UNRESOLVED RESONANCES

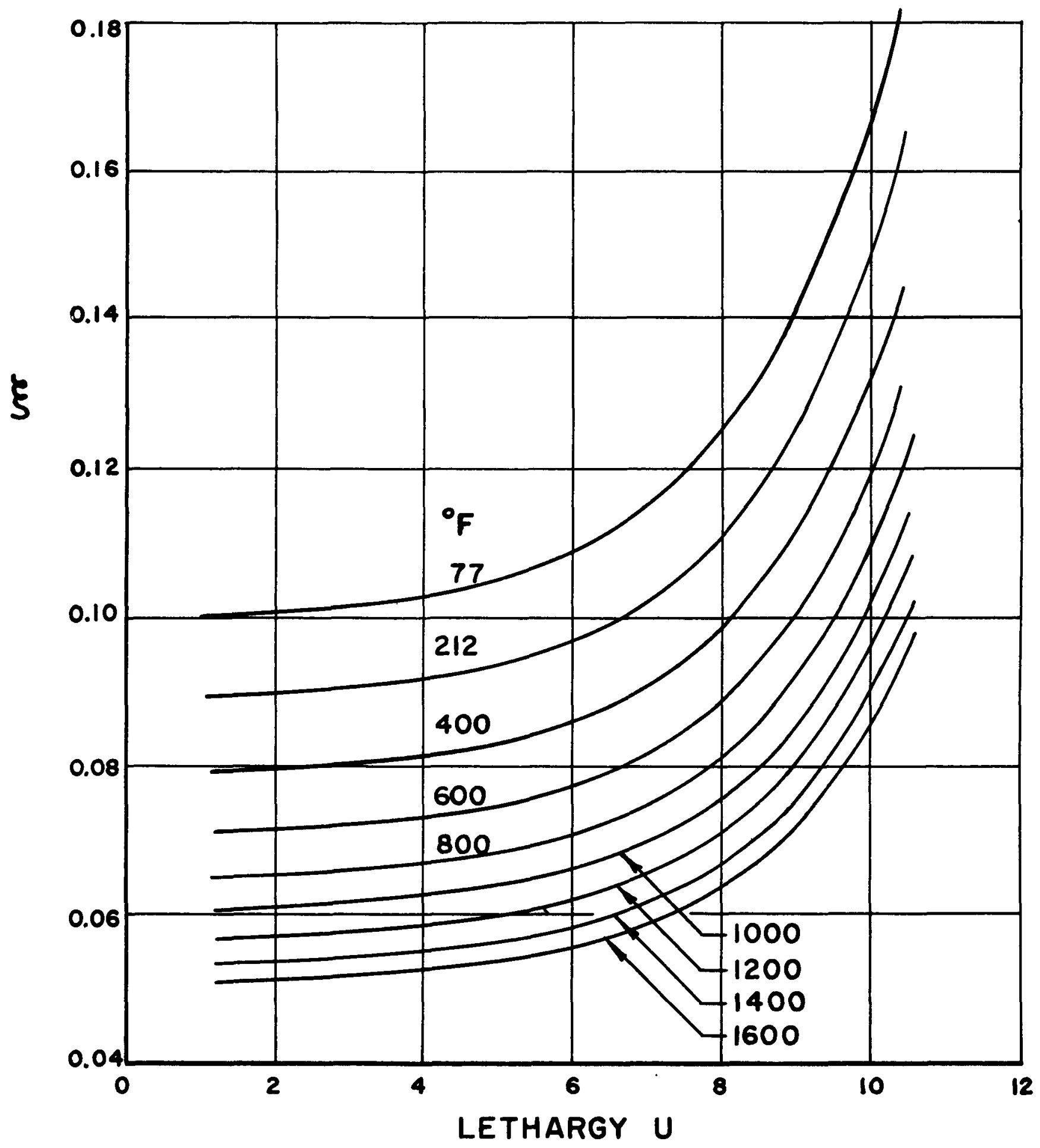

$$
1-42
$$


1. Cross-section data are rather uncertain, having been derived from graphical integration of cross-section peaks in the very low energy region, theory in the intermediate region and experimental values at high energy.

2. The values of $\xi$ are very much greater than unity, principally because $\Gamma$ is large and $\sigma_{0}$ is small, in comparison with $\mathrm{Th}-232$. For these $\xi$ values, the effective resonance integral is independent of temperature, i.e. the Doppler effect is not important. The only factor that must be accounted for is the flux self-shielding.

3. The resonance scattering in $U-233$ is very small compared with absorption:

\section{$\Gamma_{n} \ll \Gamma_{\gamma}$ and $\ll \Gamma_{f}$}

An elementary method of applying corrections to previous data is adopted in view of the uncertainties in the basic cross-sections. For any interval of energy, the ratio of $\sigma_{r e}$ to $\left(\sigma_{r e}\right)_{\infty}$ in an element with negligible resonance scattering may be written

$\frac{\sigma_{r e}}{\left(\sigma_{r e}\right)_{\infty}}=\frac{\int 1+\frac{\gamma}{\xi} \frac{\sigma_{a}}{\sigma_{p}} \frac{d E}{E}}{\int \sigma_{a} \frac{d E}{E}}=\frac{\left.\frac{\sigma_{a}}{1+\frac{\gamma}{\xi} \frac{\sigma_{a}}{\sigma_{p}}}\right]_{\bar{E}}^{\frac{\Delta E}{E}}}{\left.\sigma_{a}\right]_{\bar{E}} \frac{\Delta E}{E}}$

where $\vec{E}$ is an appropriately chosen value of energy within the interval. If the interval is very small, as in one of a 59 group sequence, E may be chosen as the energy of the midpoint and

$$
\frac{\sigma_{r e}}{\left(\sigma_{r e}\right)_{\infty}} \simeq \frac{1}{1+\frac{\gamma}{5} \frac{\sigma_{a}}{\sigma_{p}}}=f
$$

Thus the self-shielding factor is readily determined. 


\subsubsection{Discussion of Values of Capture to Fission Ratio for U-233}

In an attempt to compare the cross-sections for U-233 developed in Section 1.2-2 with those used by ORNL (22) it was found that the value $a\left(\sigma_{c} / \sigma_{f}\right)$ differed greatly in most of the high energy neutron groups. This uncertainty in a reflects the difficulty encountered in determining $\sigma_{c}$ for the fuel. The value of the capture cross-section used in the multigroup calculations has a profound effect on whether or not breeding can be achieved in a small reactor that is controlled by non-productive control rod neutron captures. Since approximately $40 \%$ of the fissions are in the epithermal range, a ratio of capture to fission (above thermal) of 0.4 or 0.5 will limit a reactor to at best, a good converter.

In order to show a comparison of the values of a presented in (22) and those used in the survey, Figure 1.2-6 was prepared. The effect of using a from reference $(22)$ is discussed in Section 3.0. Several points in the survey were recalculated with the different values of $a$, but using the fission cross-sections shown in Table 1.2-8. These are plotted along with tiet results of the survey. 


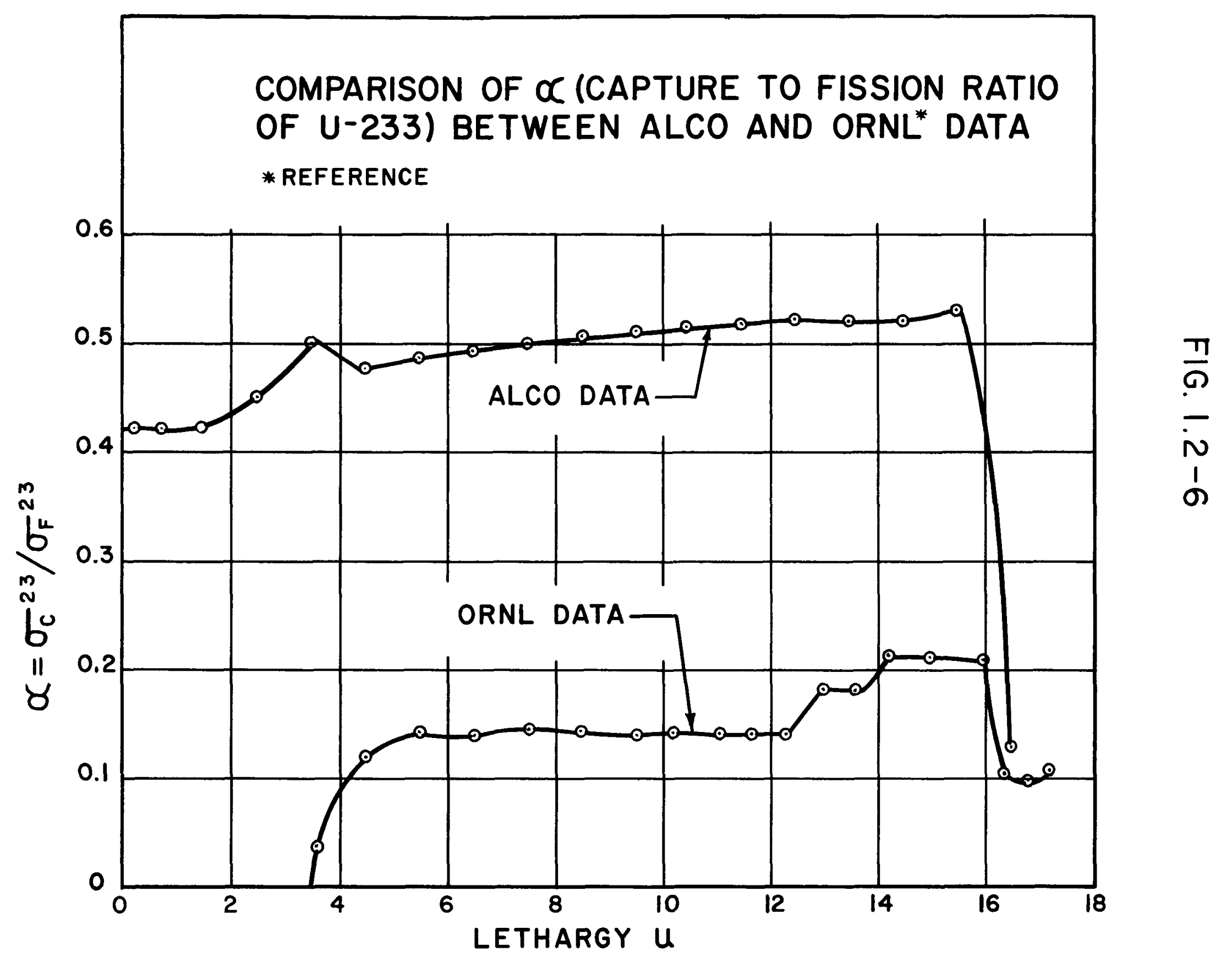




\section{$1.2,5$ Thermal Neutron Spectrum and Thermal Properties}

Since the core is heavily loaded with high thermal neutron absorbing materials, these materials can be expected to harden the thermal neutron spectrum to some extent.

Since a hardened spectrum has the most probable neutron velocity at some neutron energy above the $k T$ energy as opposed to a Maxwell Boltzman distribution, the amount of spectrum hardening is essential to determining the proper thermal neutron cross-sections.

The nuclear parameter survey covered a wide range of core loadings, and it would have been too time consuming to determine the most probable neutron velocity for each case. Therefore, a representative loading was selected. The neutron velocity spectrum was determined by the method presented by Cohen in reference (21).

The neutron spectrum in a heavy moderator in the presence of an absorption cross-section with a $1 / v$ dependence can be expressed as

$$
x^{\prime \prime}(X)+\left(2 X^{2}-1\right) N^{\prime}(X)+(4 X-\Delta) N(X)=0
$$

where

$$
\begin{aligned}
& \Delta=\frac{2 M \sum_{a}(k T)}{\sum_{s}(k T)} \\
& x=\frac{\text { velocity }}{\text { velocity at energy } k T}
\end{aligned}
$$

and $N(X)$ is number of neutrons per unit of $X$.

For the average core under consideration $\Delta$ has a value of about 2.8. Figure $(1.2-7)$ shows the neutron velocity spectrum for this case. From the curve it can be seen that the peak, or most probable neutron velocity is approximately 1.5 times the $k T$ value. Since for the PBR, the average temperature was assumed to be $1200^{\circ} \mathrm{F}$. (0.08 ev) the energy at the most probable velocity is $0.18 \mathrm{ev}$. In order to avoid numerous numerical integrations of the spectrum weighted by the individual cross-sections, the spectrum was as sumed to be similar in shape to a Maxwell-Boltzman. Therefore the thermal cross-sections were evaluated at .18 ev and corrected by $\sqrt{\pi} / 2$. These cross-sections are presented in Table 1.2-13, in addition to those obtained at $77^{\circ} \mathrm{F}$ and $1400^{\circ} \mathrm{F}$ in a similar manner. 
FIG. I.2-7 THERMAL NEUTRON VELOCITY SPECTRUM

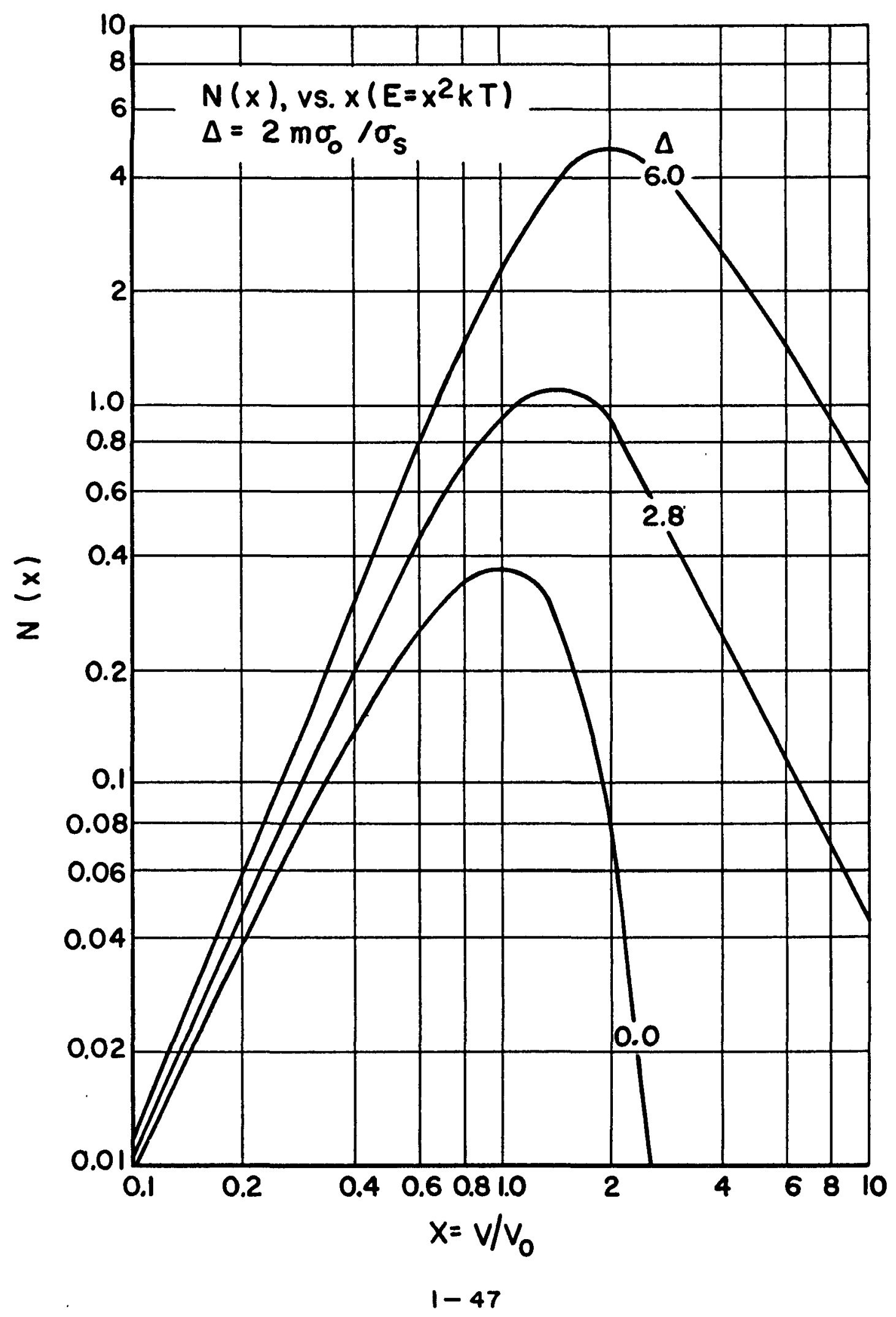




\section{THERMAL PARAMETERS}

Parameter +

$\sigma_{a}^{02}$ barns

$\sigma_{\mathrm{a}}^{23}$ barns

$\sigma_{a} c$ barns

$\sigma^{\circ}{ }^{\circ}$ barns

$\sigma_{a}{ }^{B}$ barns

$\sigma_{\mathrm{a}}{ }^{13}$ barns

$\sigma_{\mathrm{a}}^{\mathrm{xe}} \times 10^{18}$

$\sigma_{s} 02$ barns

$\sigma s^{23}$ barns

$\sigma_{s}{ }^{c}$ barns

$\sigma_{\mathrm{s}}{ }^{\circ}$ barns

$\sigma_{f^{23}}$ barns

2) 23
Temperature ${ }^{\circ} F . *$
$77^{\circ} \mathrm{F}$
$1200^{\circ} \mathrm{F}$.
$1400^{\circ} \mathrm{F}$.

4.431

2.477

2. 346

349.72

195.5

185.2

0.00190

0.00106

0.00101

0

0

0

449.

251.

238.

90.34

50.50

47.84

2.20

1.049

0.910

11.6

11.6

11.6

13. 3

13.3

13.3

4.8

4.8

4. 8

4. 2

4.2

4.2

316.45

176.9

167.6

2.52

2.52

2.52

+ All microscopic absorption cross-sections are averaged over a Maxwell Boltzmann at the appropriate energy.

* From Section 1.2.5, the energy at which the crosssections are evaluated is $2.25 \mathrm{kT}$. 


\subsubsection{Calculation of Breeding Ratio}

The initial breeding ratio (IBR) * $^{*}$ may be defined as the total absorption of neutrons in Th-232 per neutron absorbed in U-233 provided losses in $\mathrm{Th}-233$ and $\mathrm{Pa}-233$ are small and can be neglected. In a reactor such as the PBR where the thermal neutron flux is about $10^{14} \mathrm{n} / \mathrm{cm}^{2}-\mathrm{sec}$ the losses are small $(\sim 2 \%)$ and the assumption is justified.

Since fast neutron absorptions have a significant effect on the IBR, the neutrons absorbed in each energy group of the fast range as well as those absorbed thermally were considered. Therefore, if we define:

$$
\begin{aligned}
& \mathrm{H}=\bar{\phi}_{t h} \sum_{t h}^{02}+C \sum_{n=1}^{54} \bar{F}_{n} \Delta_{n} \sum_{a n}^{02} \\
& J=\bar{\phi}_{t h} \sum_{t h}^{23}+C \sum_{n=1}^{54} \bar{F}_{n} \Delta_{n} \sum_{a n}^{23}
\end{aligned}
$$

where the factor $C$ is defined as

$$
c=\bar{\phi}_{f} / \sum_{n=1}^{54} \bar{F}_{n} \Delta_{n}
$$

This is the conversion factor between the actual spatially averaged value of the total fast flux and the total relative value. The actual fast flux was determined from the power level of the reactor as shown in equations $(1,2-26)$ and $(1,2-27)$. The fluxes reflect thermal absorptions in the control rods as all cores were poisoned out before the coupling coefficient was calculated.

From the above definitions, the breeding ratio in the core without a blanket may be calculated by

$$
\operatorname{IBR}(\operatorname{core})=\frac{\mathrm{H}}{\mathrm{J}}
$$

* In this report, the term breeding ratio is used regardless of whether its value is above or below unity. 
The effect of a blanket may be included by adding the total absorptions in the Th-232 in the blanket to that in the core and dividing by the total absorptions in the $\mathrm{U}-233$ in the core. If $\mathrm{V}_{\mathrm{c}}$ and $\mathrm{V}_{\mathrm{b}}$ are the volumes of the core and blanket respectively, the overall IBR may be written:

$$
\operatorname{IBR}\left(\text { total) }=\frac{\mathrm{H}_{\mathrm{C}} \mathrm{V}_{\mathrm{C}}+\mathrm{H}_{\mathrm{b}} \mathrm{V}_{\mathrm{b}}}{\left(\mathrm{J}_{\mathrm{c}} \times \mathrm{V}_{\mathrm{c}}\right)}\right.
$$

\subsubsection{Results of Nuclear Parameter Survey}

The principal items of interest in the PBR are the initial breeding ratio and the effective multiplication factor. If the combination of uranium, thorium, graphite and void $\exists$ re such that the conversion ratio is small or $\mathrm{K}_{\mathrm{eff}}$ is less than unity, then the cores are of little interest, but they do indicate the general trend. On the other hand, cores with $\mathrm{K}_{\text {eff }}$ above unity and a reasonably high breeding ratio are very desirable. Often the dividing line is very fine. Therefore, the basic results of the survey have been expressed graphically as plots of $\mathrm{K}_{\mathrm{eff}}$ and IBR versus atom ratios of thorium to uranium for various void fractions, weight per cent total oxide in the fuel balls and core diameters.

In order to lend added significance to the general results, a series of cross plots are presented which include the following:

1) Initial breeding ratio versus void fraction for $7,9,12,16$ and $20 \mathrm{ft}$. core diameters and for oxide weight percents of 10 and 20 with $K_{\text {eff }}$ equal to 1.00 and 1.10 .

2) Initial breeding ratio versus core diameter for several void fractions with $K_{\text {eff equal to }} 1.00$ and 1.10 .

3) Critical loading of U-233 vs core diameter for several void fractions.

\subsubsection{Single Region Core}

It was realized at the beginning of the survey that large diameter cores would have a higher breeding ratio due to reduced neutron leakage. The limit on core diameter was set at twenty $\mathrm{ft}$. due to the pressure vessel size. Core diameters were thus considered starting at twenty $\mathrm{ft}$. and going to seven $\mathrm{ft}$. The smaller cores were expected to have a lower breeding ratio, but it was planned to surround them with a fertile blanket and thus improve the breeding. 
The following set of curves (Fig. 1.2-8 through 1.2-17) show the results of the survey, assuming an operating power level of $350 \mathrm{tMW}$ and equilibrium xenon. The curves indicate that where the breeding ratio is high, $K_{e f f}$ is low and vice versa. It should be noted that as the volume fraction of void decreases, $K_{\text {eff }}$ increases. Since there is always $39 \%$ void associated with the ball volume, to decrease the void some of the ball volume must be replaced by solid graphite. Thus the increase in $K_{\text {eff }}$ with additional graphite reflects the tendency for the cores to be under moderated. The variation of IBR with void for constant $\mathrm{K}_{\mathrm{eff}}$ also reflects the undermoderation of the core. By decreasing the void, a higher atom ratio of $\mathrm{Th} / \mathrm{U}$ can be used which increases the IBR. As the void is further decreased, the reduction causes the thermal flux to increase, which increases xenon and carbon neutron captures and also the leakage. The overall effect of void fraction on IBR is shown in Figs. 1.2-18 and 1.2-19 for a $\mathrm{K}_{\mathrm{eff}}$ of 1.00 and 1.10 respectively.

It should be pointed out again that each core was poisoned out to make $K_{e f f}$ equal to unity before the IBR was calculated. Thus the IBR reflects the loss of neutrons to control rod captures.

The core loading has a pronounced effect on the feasibility of any reactor design. Cores requiring upwards to $1000 \mathrm{~kg}$ of $\mathrm{U}-23.3$ are practically out of the question unless there are drastic changes in the supply of U-233. Also, cores with larger fuel loading must support very large inventory costs which seriously hurt the economics. Therefore, Fig. 1.2-20 is presented to show the critical core loading as a function of core diameter.

\subsubsection{Small Breeder Blanket Core}

A small seven foot core was selected to determine the gain in breeding ratio by surrounding it radially with a fertile blanket. The design point is indicated on Fig. 1.2-8 by the letter A. This specific core composition was chosen because Keff was about 1.10 and thus should yield a reasonable lifetime and yet be controllable with a finite number of rods.

The blanket was composed of spherical elements containing $\mathrm{ThO}_{2}$ and graphite. The magnitude of the change in breeding ratio with increased thorium in the blanket was not known, so the weight percent of $\mathrm{ThO}_{2}$ in the balls was varied from 10 to $50 \%$. The blanket was physically separated from the core by a four-inch graphite ring. 
Using the core properties based on a homogenized model, multiregion two-group calculations were made to determine the flux distributions for the various blanket compositions. Fig. 1.2-21 shows a typical flux distribution for the case of 50 weight $\% \mathrm{ThO}_{2}$ in the blanket balls.

The flux distributions were integrated to determine the overall breeding ratios including the blanket contributions. The effect of increasing the weight percent $\mathrm{ThO}_{2}$ in the blanket balls is illustrated in Fig. 1.2-22 where IBR is plotted versus wt $\% \mathrm{ThO}_{2}$. Above 50 wt $\%$ ThO2 the IBR levels off and little is gained by increasing the concentration of thorium. Figs. 1.2-23 through 1.2-27 show the variation of some of the more detailed parameters of the fertile blanket as a function of thorium concentration.

\subsubsection{Large Breeder Blanket Cores}

Since the breeding ratio of the seven-foot core was quite low, it was decided to look briefly at the effect a blanket would have on the breeding ratio of larger cores. In order to avoid numerical integration of the blanket flux for each case, it was assumed that all the leakage neutrons were captured by thorium in the blanket. This is an upper limit, and it is realized that the overall breeding ratio will be somewhat lower than that determined by this calculation.

The neutron captures in the control rods seriously limit the breeding ratio. If the core is designed for continuous fuel loading, the $\mathrm{Th} / \mathrm{U}$ ratio can be increased until $\mathrm{K}_{\text {eff }}$ is close to unity and the neutrons previously lost to the rods can be absorbed in thorium. Therefore, the IBR for the larger cores with a blanket was determined for $\mathrm{K}_{\text {eff }}$ equal to 1.10 and 1.00. These are shown in Figs. 1.2-28 and 1.2-29. 

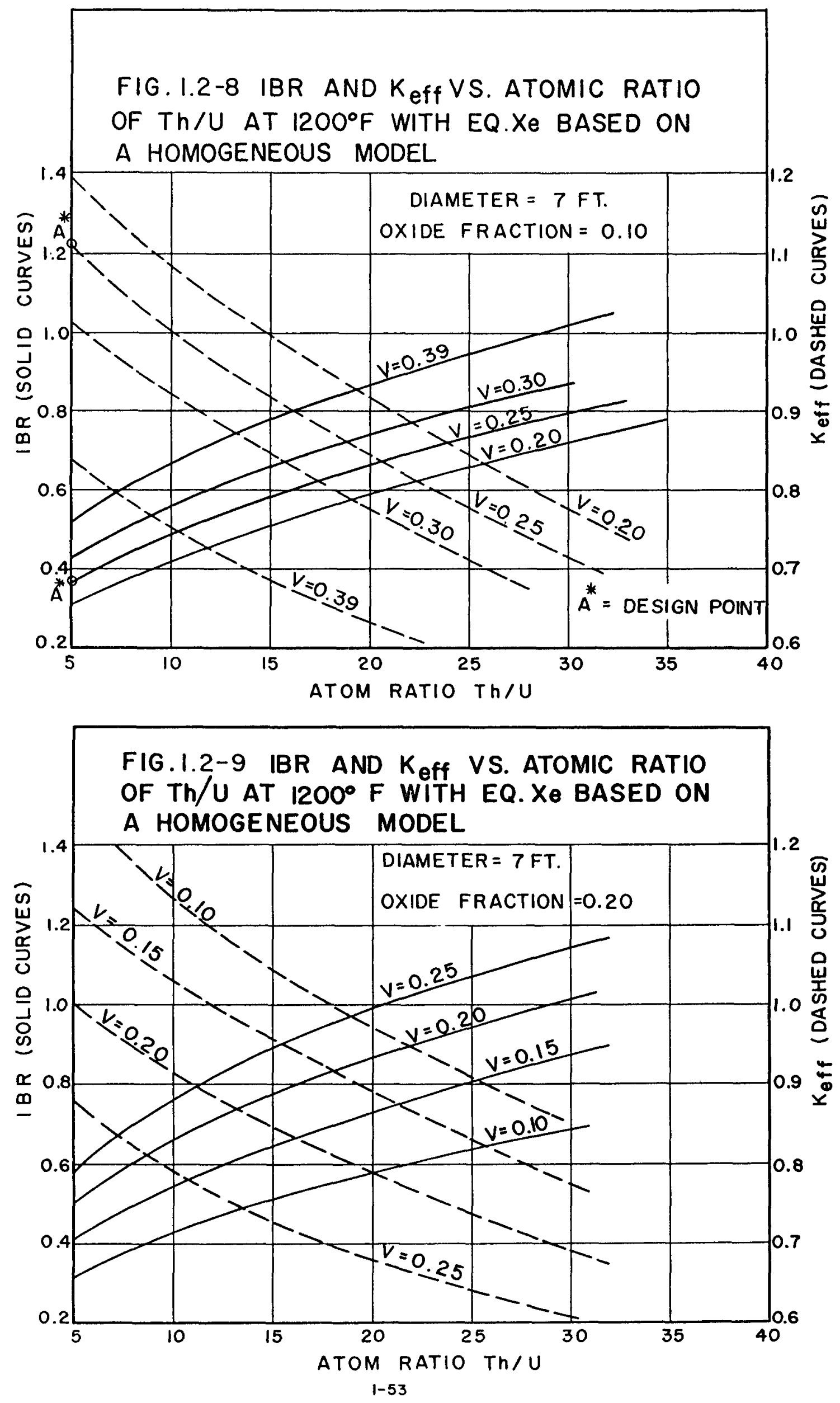


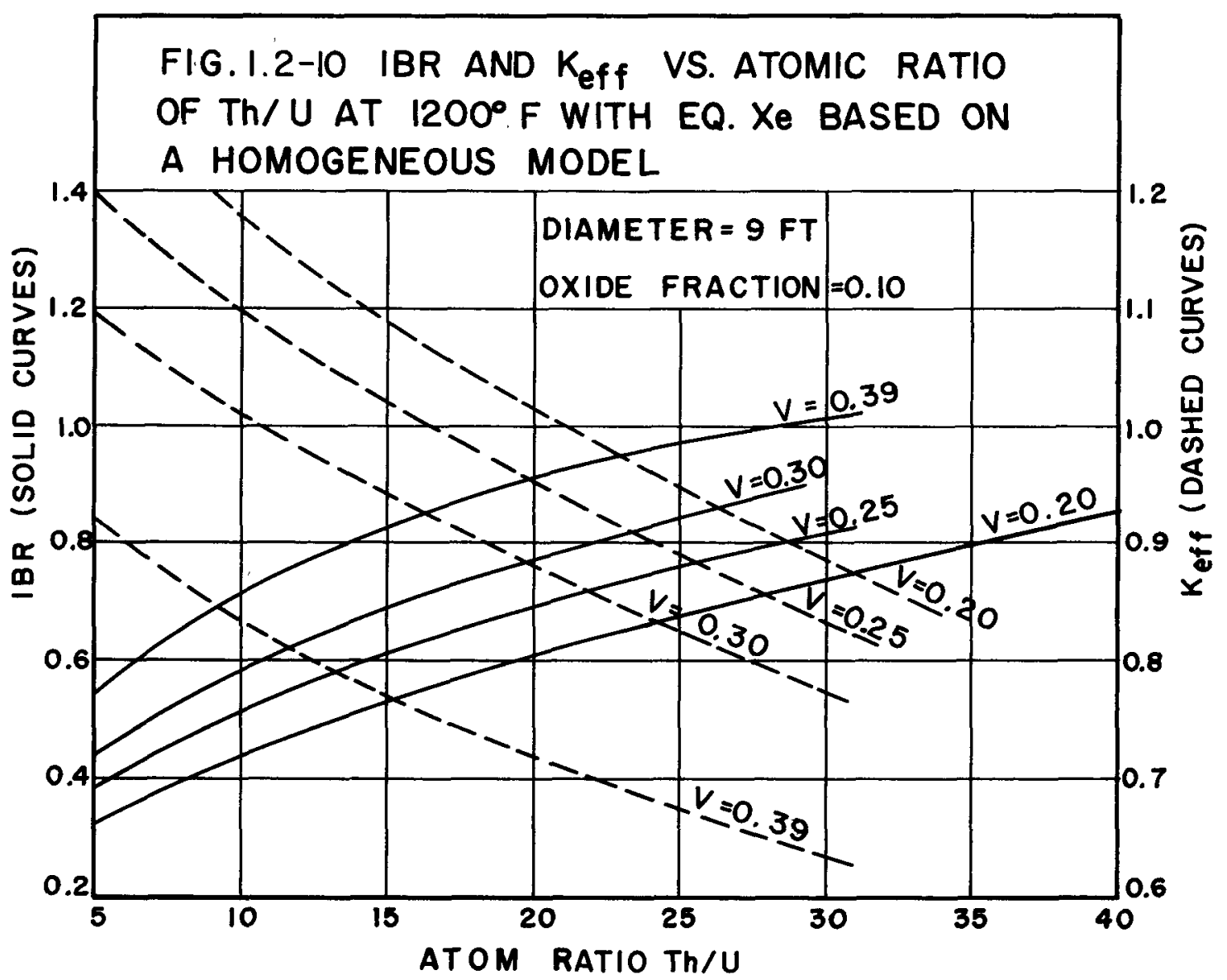

FIG.I.2-II IBR AND K Off VS. ATOMIC RATIO OF Th/U AT $1200^{\circ} \mathrm{F}$ WITH EQ.Xe BASED ON A HOMOGENEOUS MODEL

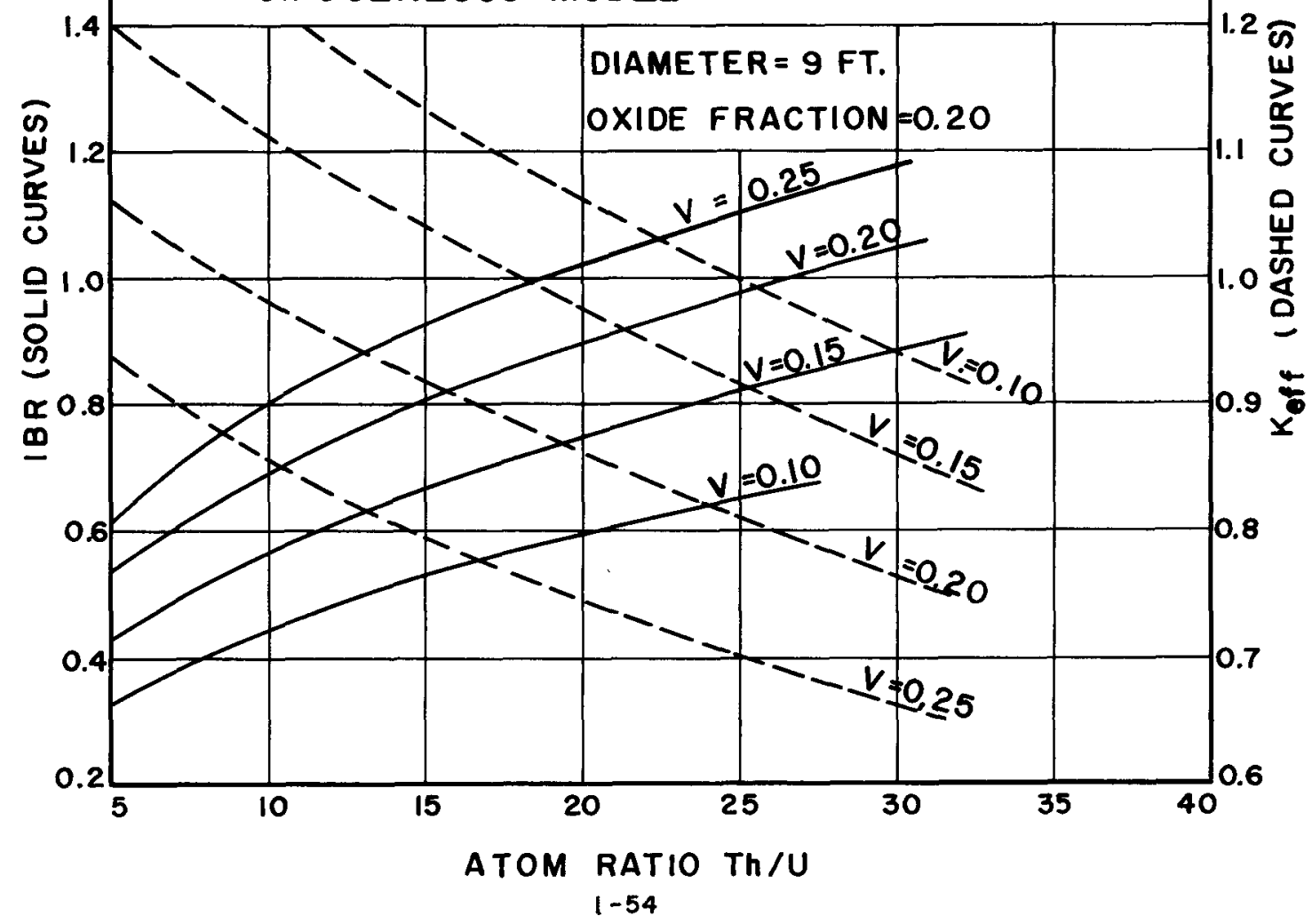



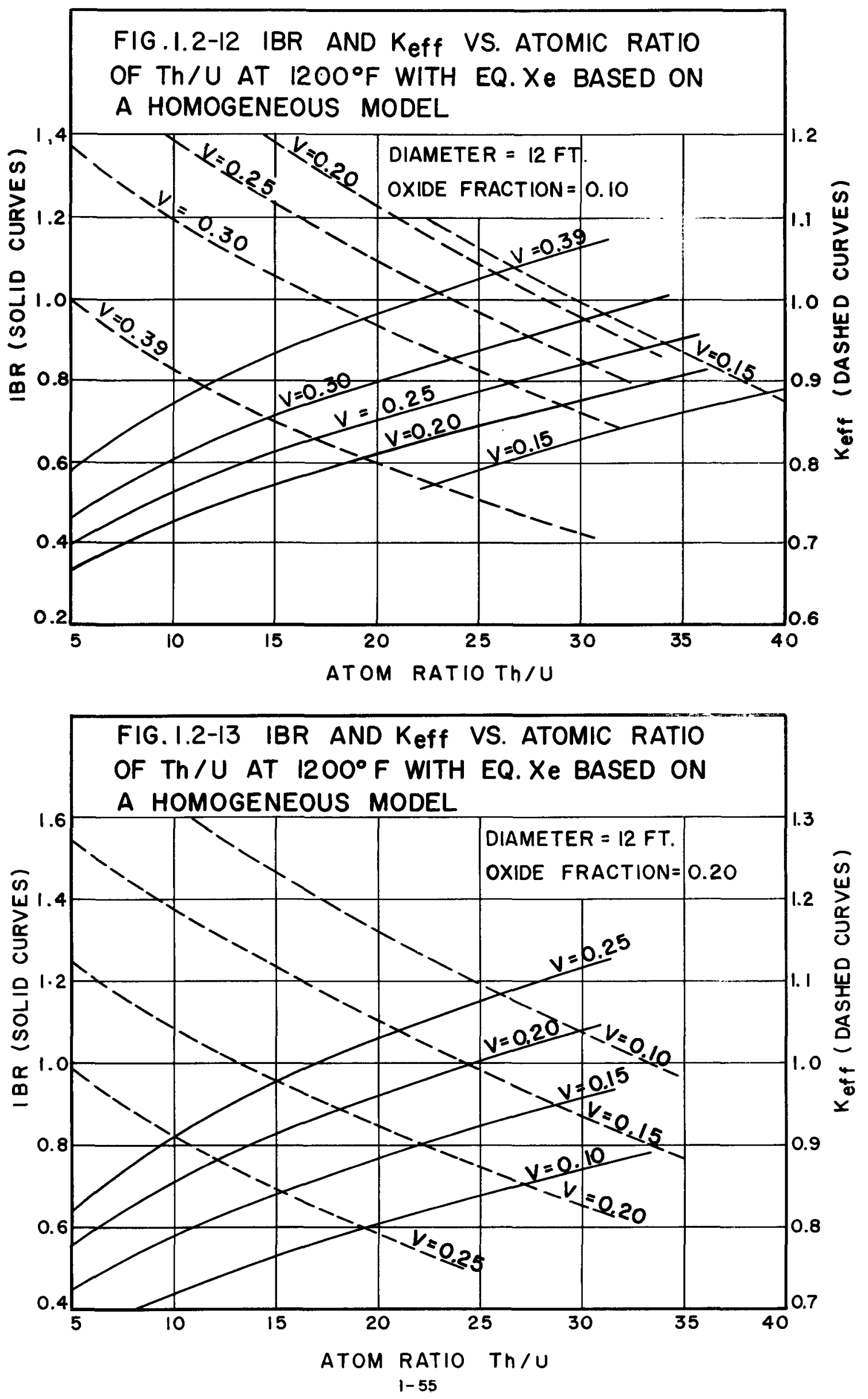

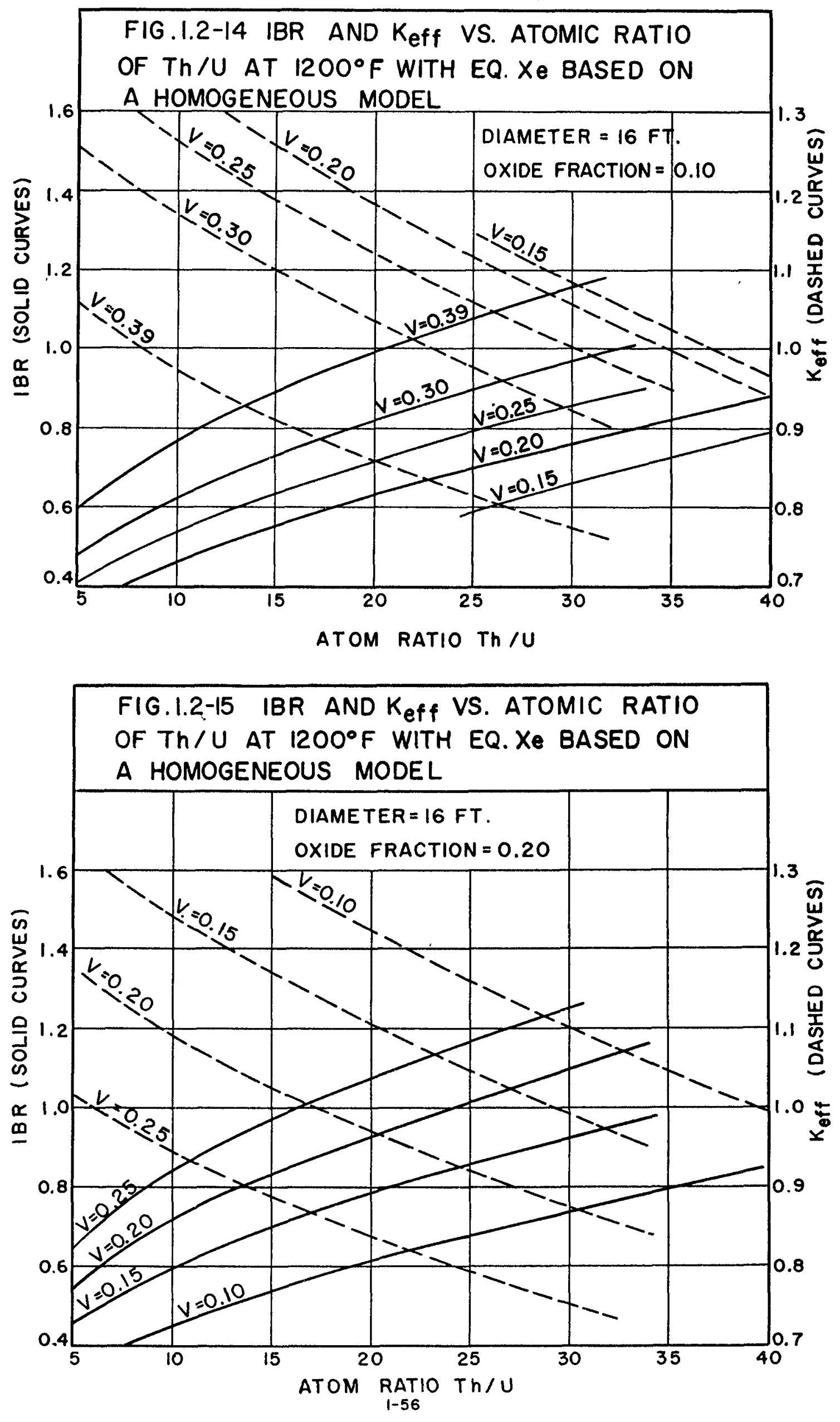

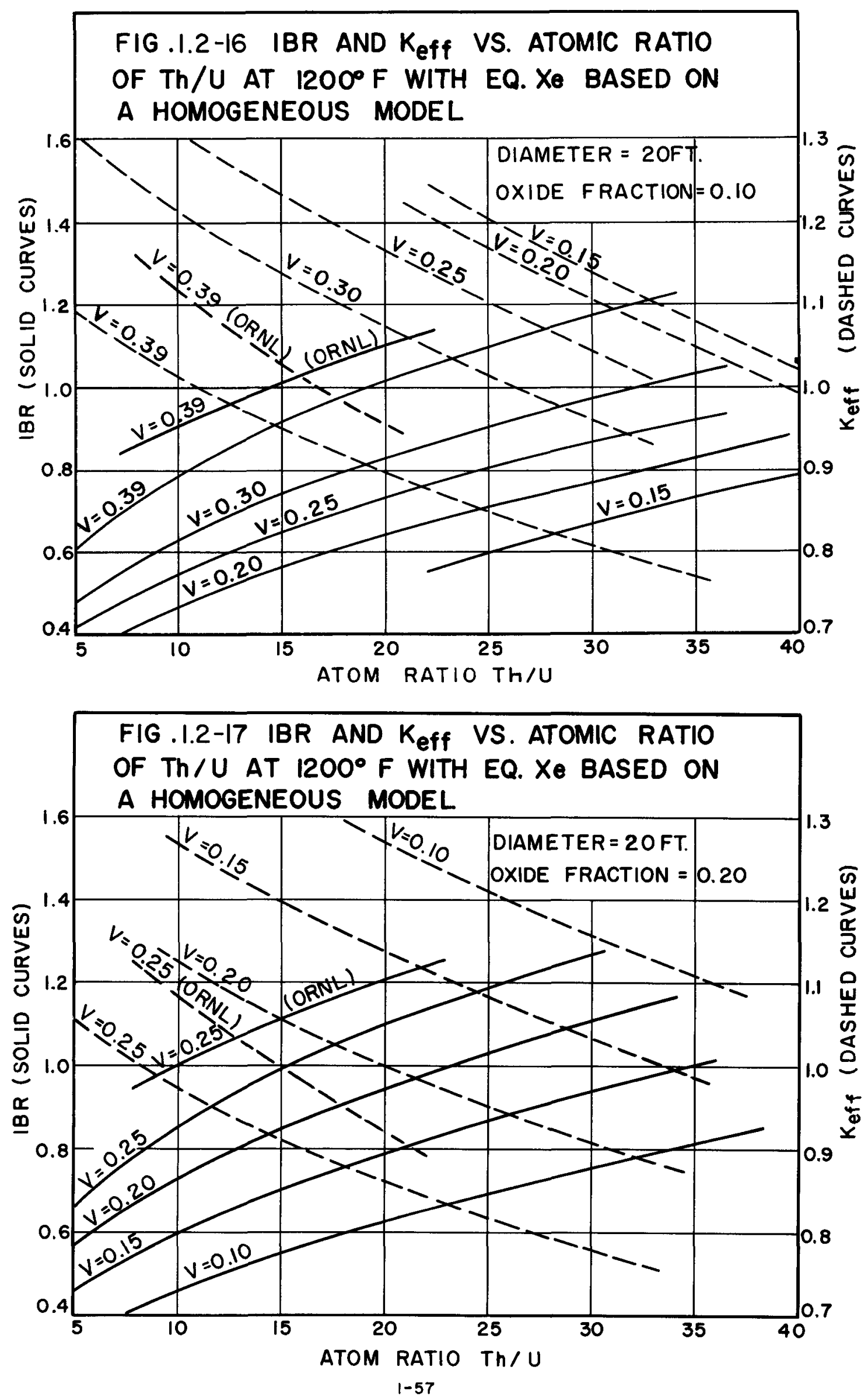

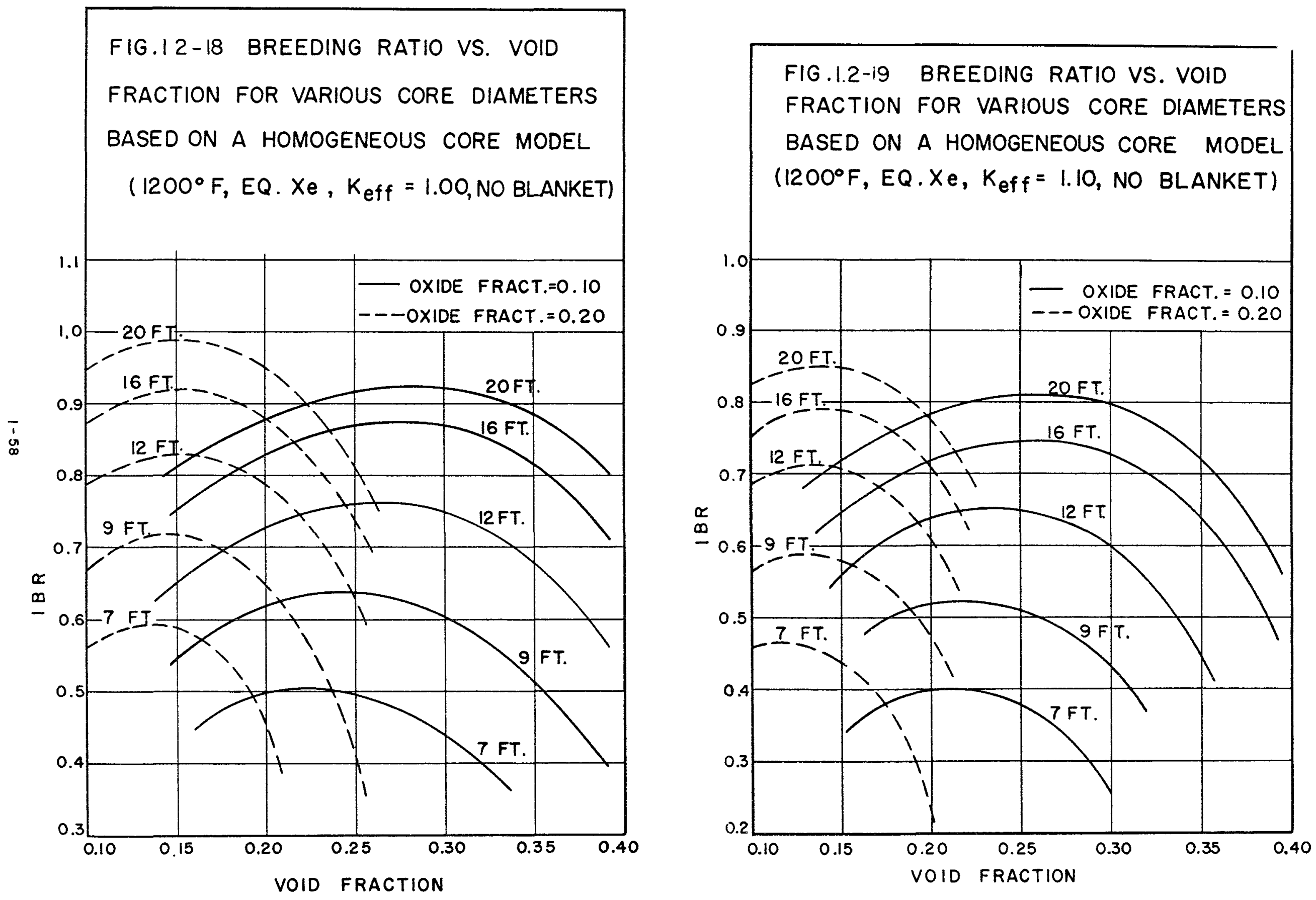

O... 


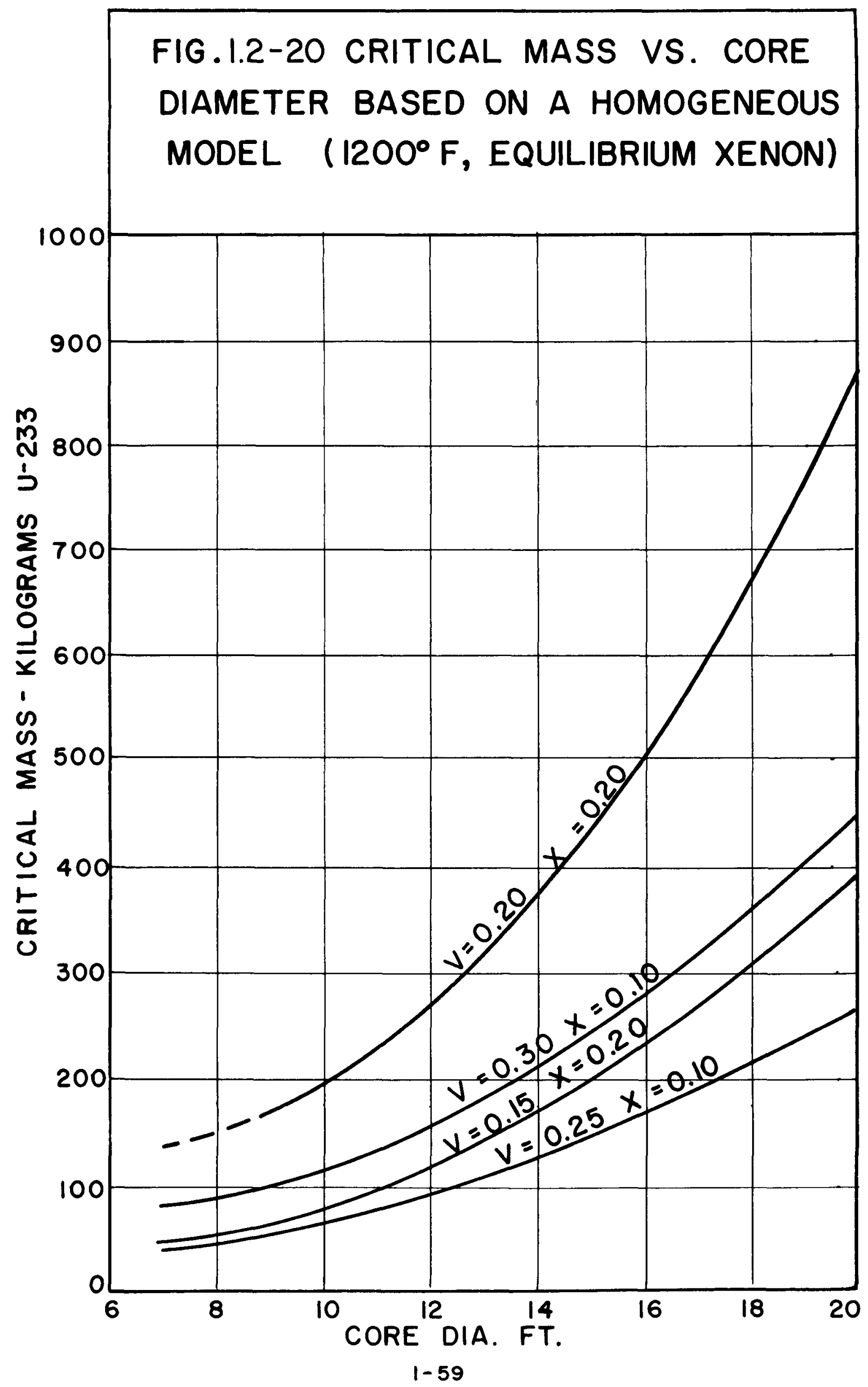


RELATIVE RADIAL FAST AND THERMAL FLUX FOR THE 7FT. CORE WITH A 16 INCH BLANKET AT $1200^{\circ} \mathrm{F}$

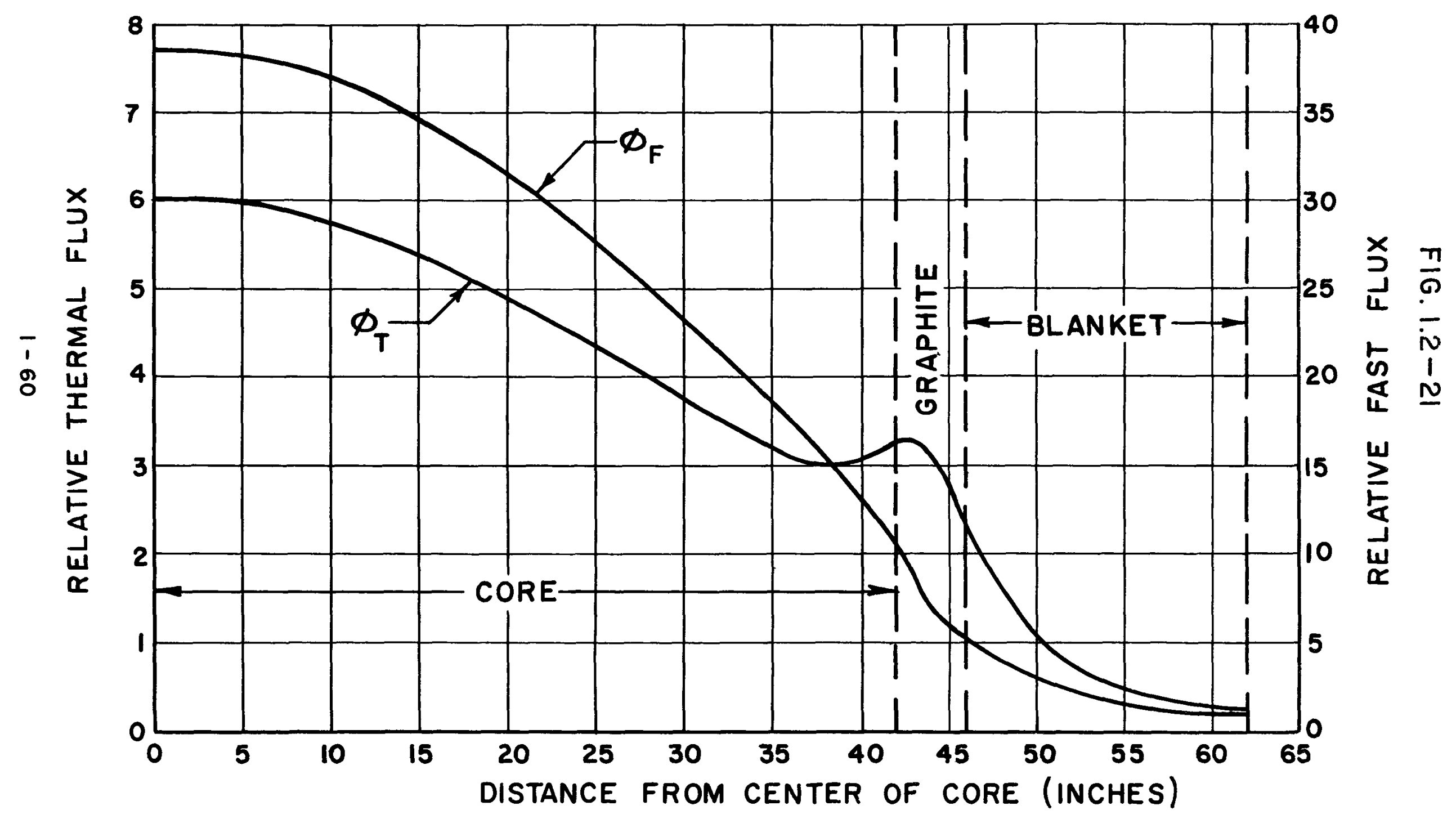




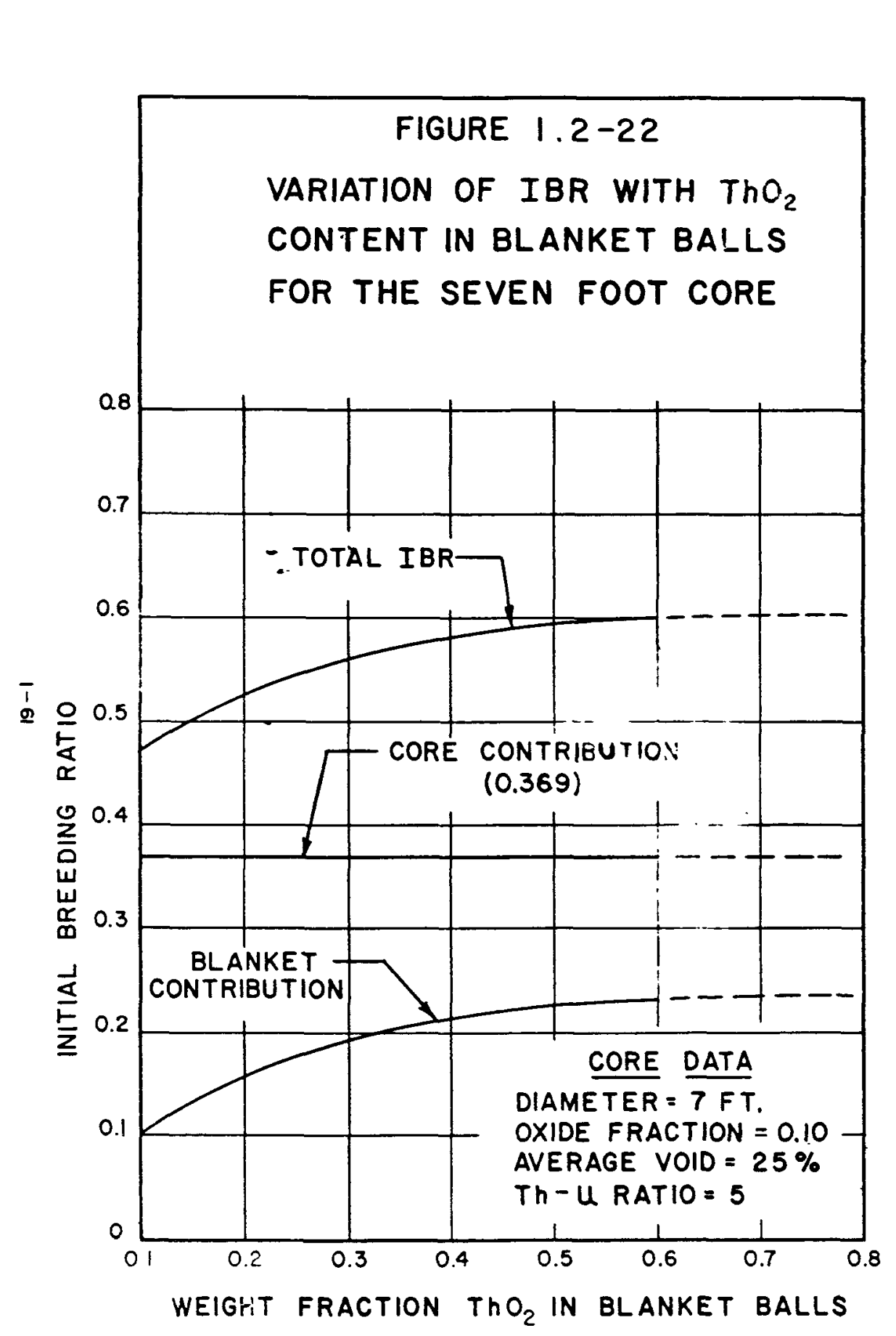

FIG. $1.2-23$

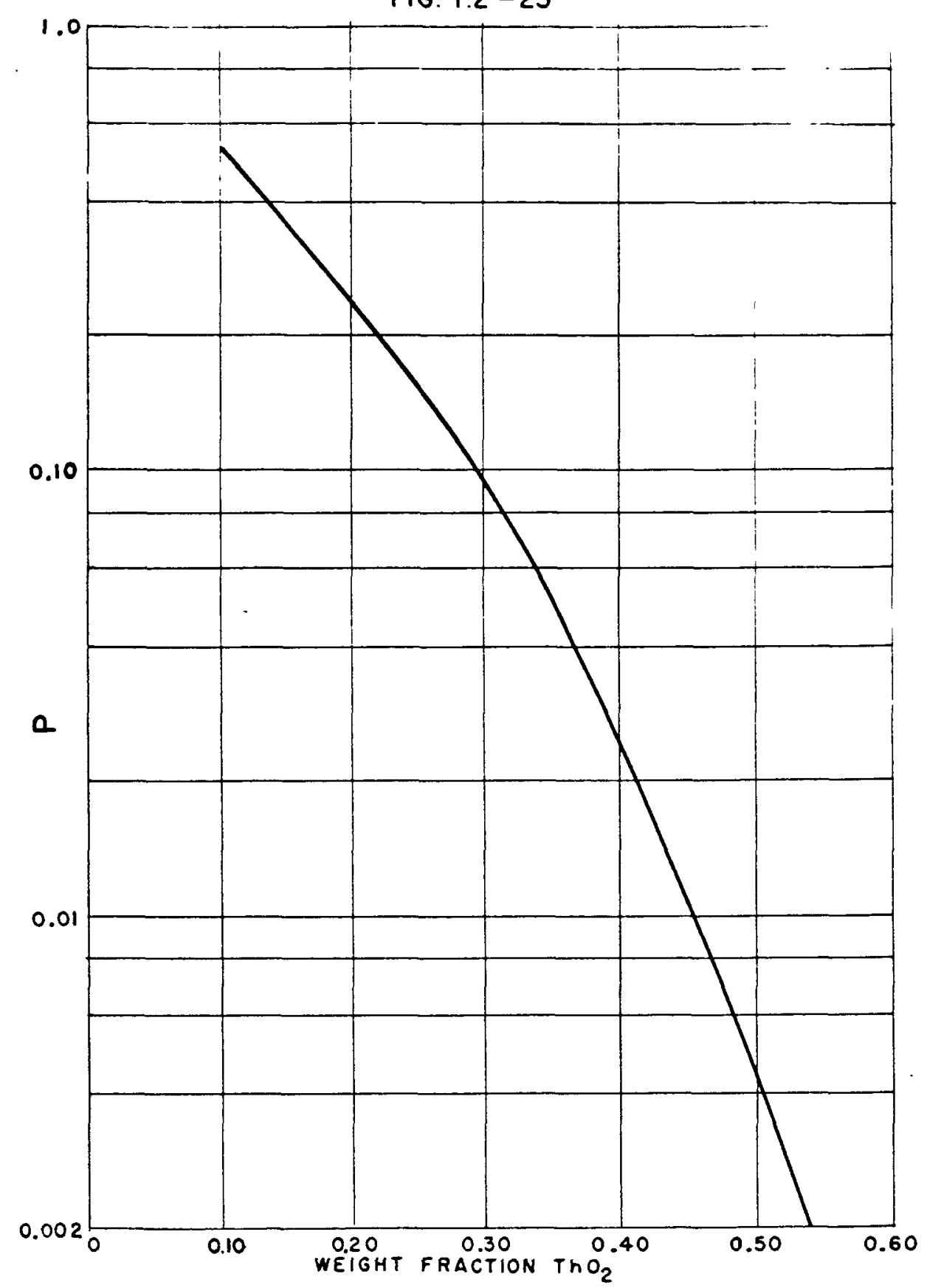

RESONANCE ESCAPE PROBABILITY $P$ VS. WEIGHT FRACTION ThO IN BLANKET (VOID $=39 \%)$ 


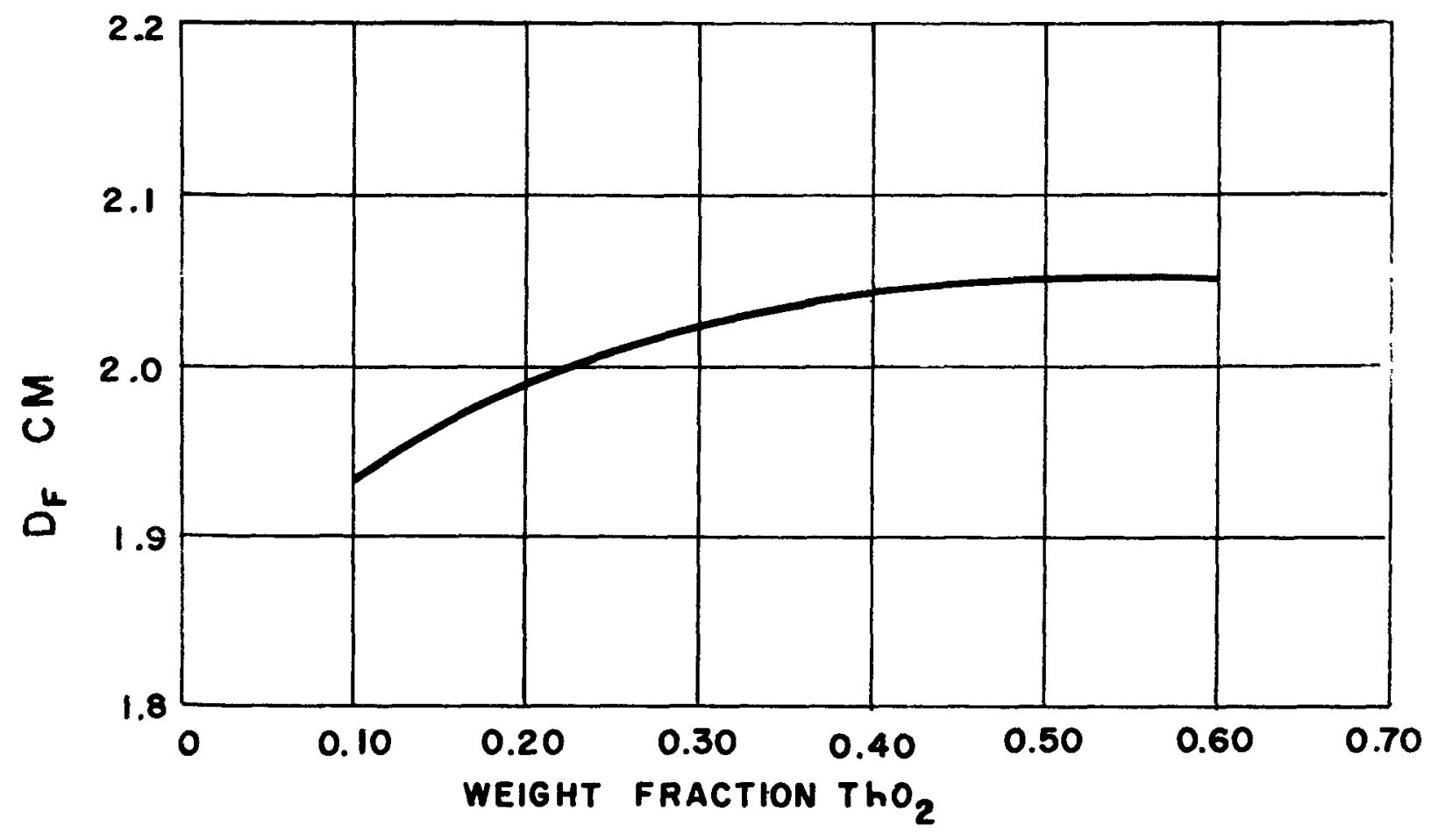

FIG. I.2-24 DF VS. WEIGHT FRACTION $\mathrm{THO}_{2}$ IN BLANKET, (VOID FRACTION $=0.39$ )

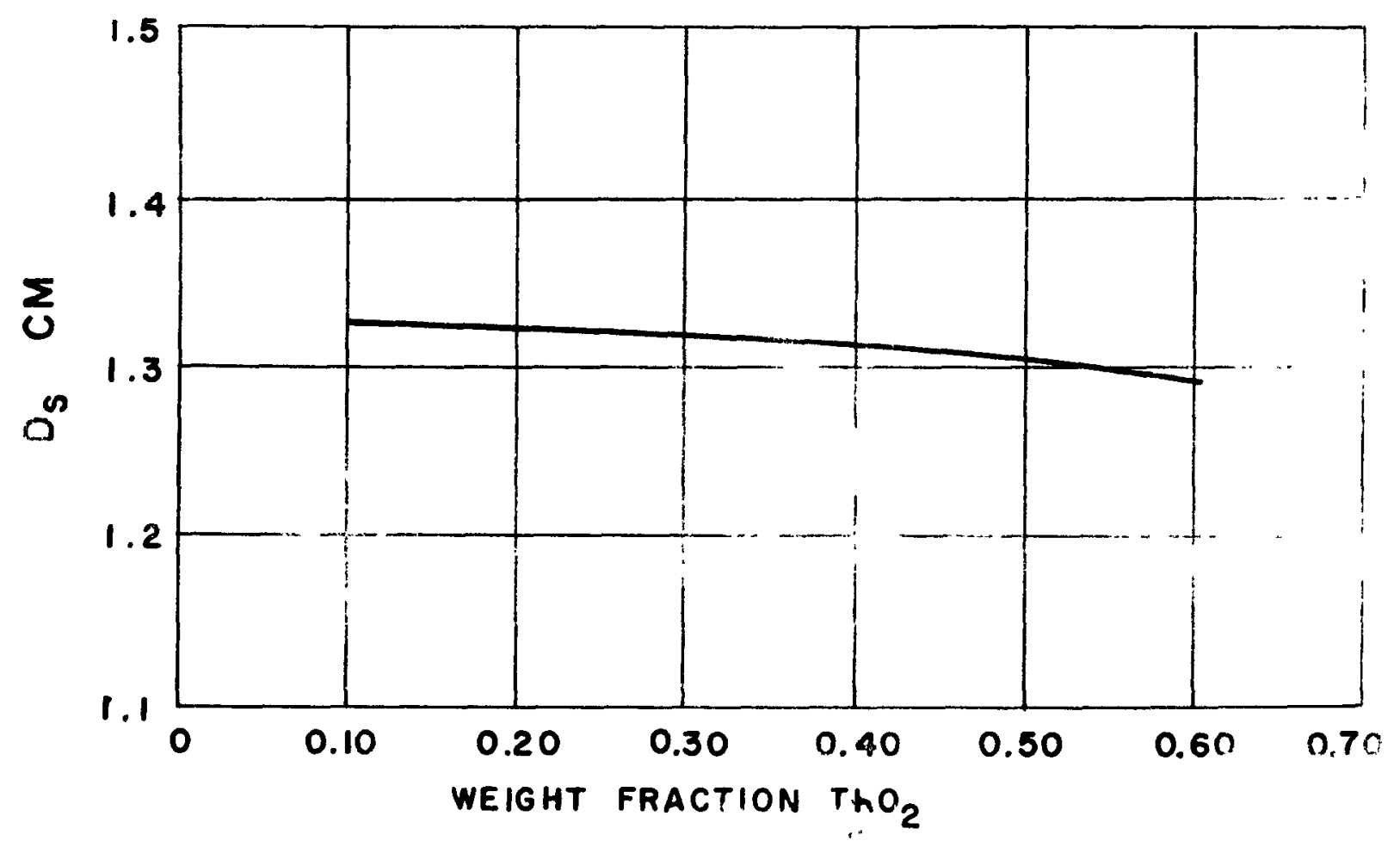

FIG.I.2-25 DS VS. WEIGHT FFIACTION $\mathrm{ThO}_{2}$ IN BLANKEI (VOID FRACTION $=0.39$ ) 
FIG. $1.2-26$

FERMI AGE $\tau$ VS. WEIGHT FRACTION Th $\mathrm{O}_{2}$ IN BLANKET. (VOID FRACTION=0.39)

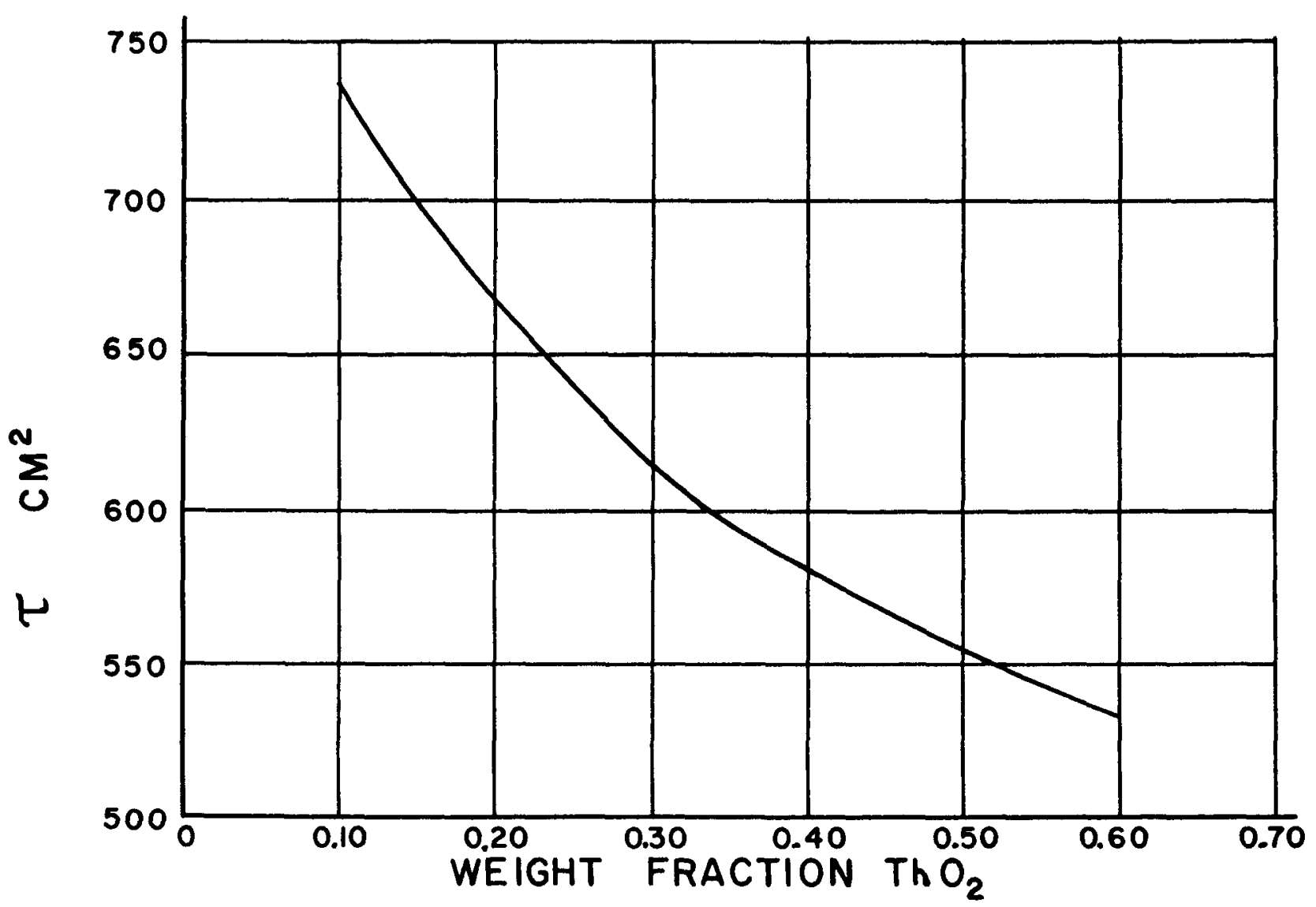

FIG. 1.2-27

AVERAGE FAST MACROSCOPIC ABSORPTION CROSS SECTION VS. WEIGHT FRACTION ThO IN BLANKET.

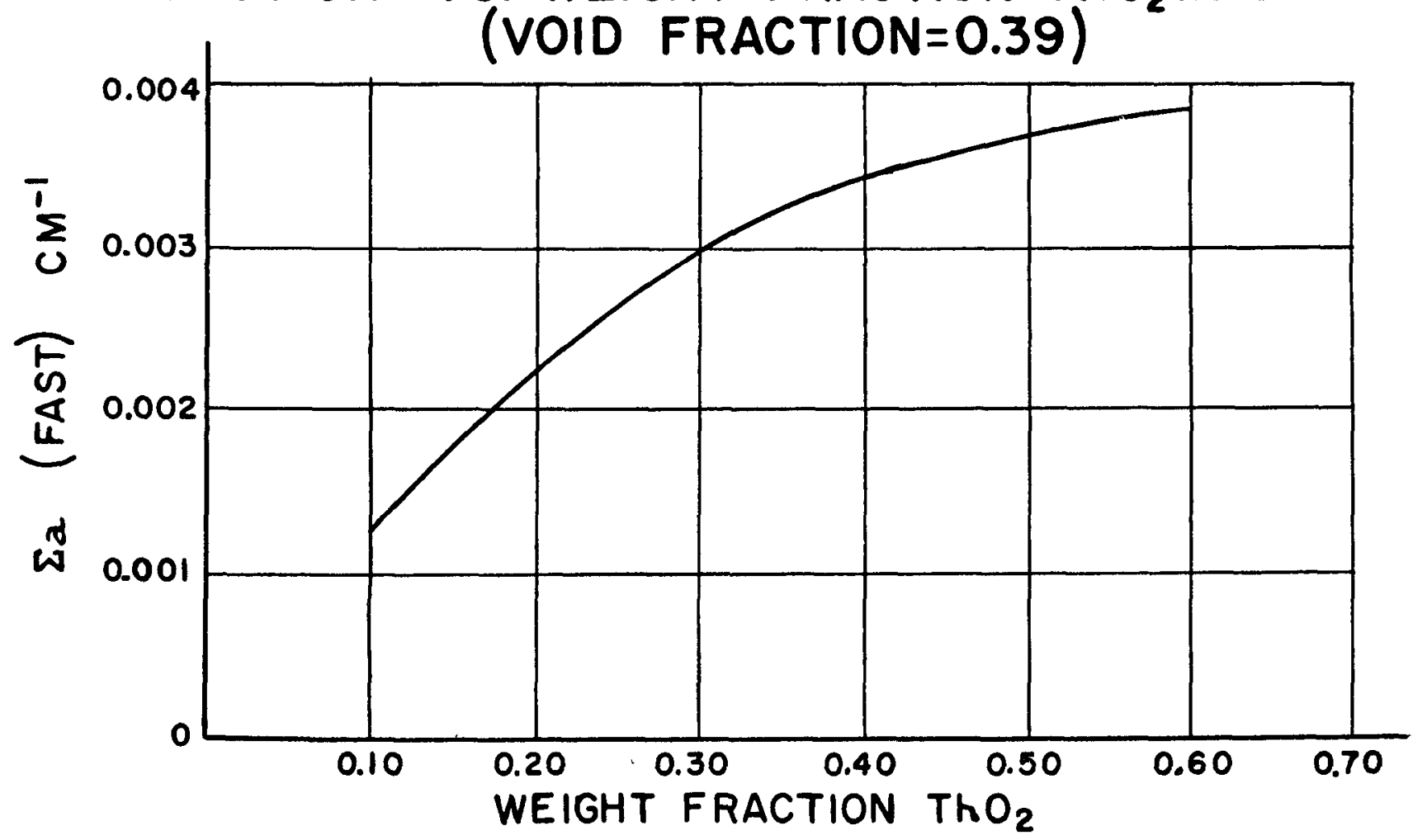



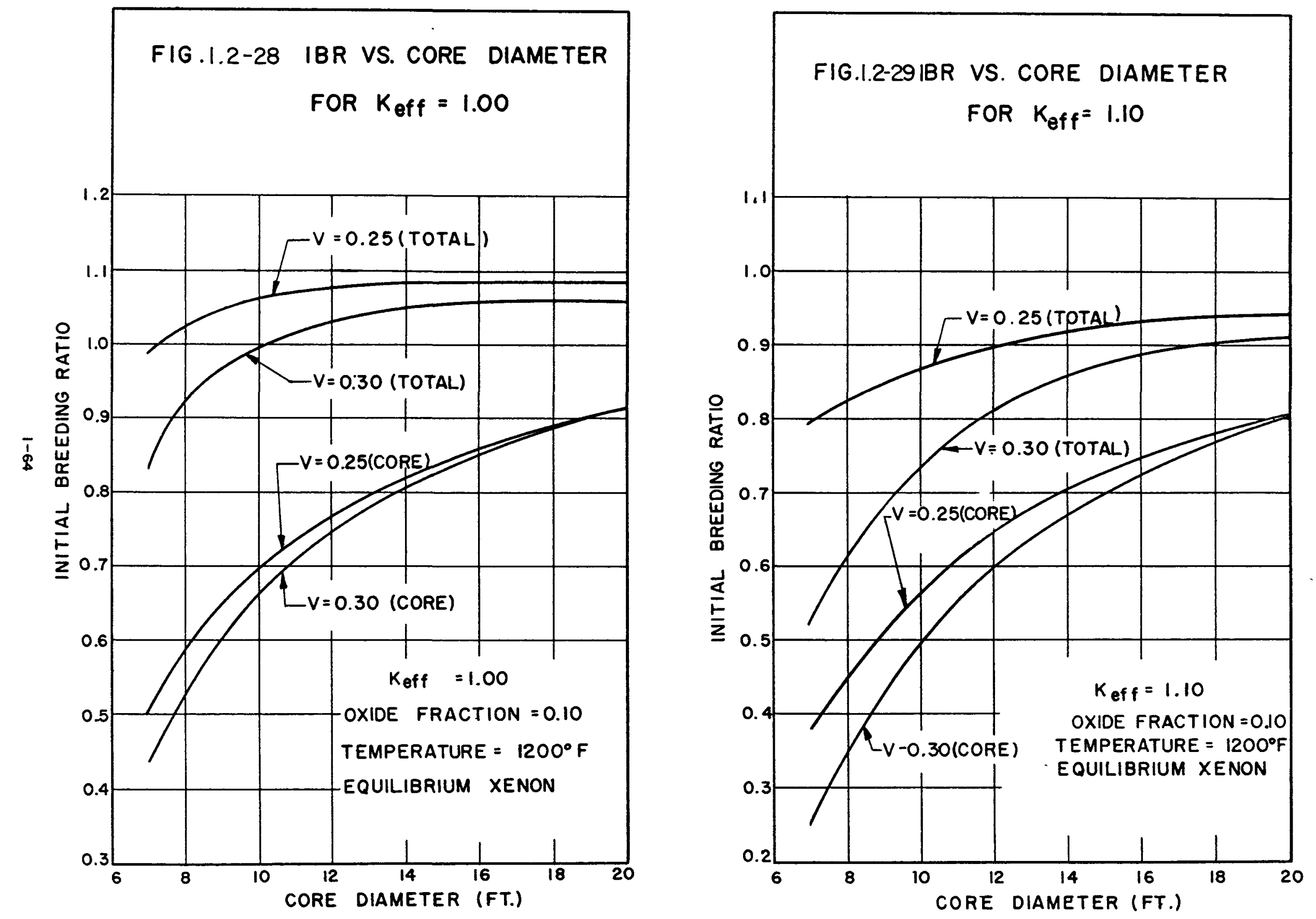


\subsection{Selection of Final Core:}

The selection of the final core was based on the factors listed below:

a) Core loading (fuel inventory cost)

b) Fuel fabrication method

c) Pumping power

d) Initial breeding ratio

e) Initial $\mathrm{K}_{\mathrm{eff}}\left(1200^{\circ} \mathrm{F}\right.$, eq. Xenon)

The critical mass for large cores of the order of 12 to 20 feet in diameter is in the range of a hundred to about a thousand $\mathrm{kg}$. of U-233 as seen in Fig. 1.2-20. The inventory cost alone on this amount of fuel is quite large and in addition, there is the problem of securing or building up very large amounts of $U-233$; therefore, the large cores were disqualified.

Fuel elements can be fabricated by impregnation, modular dispersion or lumping. Limitations on loading by these methods will probably fall between 10 and $50 \%$ by weight fis sile and fertile material. In order not to make the design restrictive as to method of fuel element manufacture a limit of 10 weight percent oxide was used in the final design.

While fuel inventory and capital costs for the graphite moderator and the reactor pressure vessel place an upper limit on core size, pumping power imposes a lower limit. It was felt that $1-1 / 4$ percent of the reactor power could be expended as core pumping power without seriously imparing the reactor economics. With this limitation, the minimum core diameter with a $39 \%$ void fraction is 8 feet. At larger diameters, smaller void fractions can be used and still not exceed the pumping power limit. Table 1.3-1 lists the void fraction corresponding to a given core diameter for constant pumping power. 
TABLE $1.3-1$

\title{
COMBINATION OF VOID FRACTION AND CORE DIAMETER
}

\author{
FOR 1.25 PERCENT CORE PUMPING POWER
}

Diameter (Ft.)

8

$81 / 2$

9

$91 / 2$

10
Void (Percent)

39.0

34.0

29.0

25.5

21.5

In order to obtain a reasonable lifetime from each core fuel loading, it was deemed necessary to select a core with an initial $\mathrm{K}_{\mathrm{eff}}\left(1200^{\circ} \mathrm{F}\right.$ eq. xenon) of about 1.10. From Fig. 1.2-19 of the results of the nuclear survey, it can be seen that the smallest core which meets the pumping power consideration of Table 1.3-1 and still has a reasonable IBR is $9 \mathrm{ft}$. in diameter and has a $29 \%$ void. A smaller core requires a larger void which reduces the IBR drastically. Larger cores have a higher associated IBR, but the blanket volume (for constant thickness) increases rapidly with core diameter and this affects the overall economics adversely. Thus the $9 \mathrm{ft}$. core with the $29 \%$ void was selected.

With ile above parameters fixed, it was feasible to apply Doppler broadening and self-shielding factors to the cross-sections to improve the accuracy of the results. The correction factors were based on a void of 29 percent, an oxide weight percent in the balls of 10 and an average $\mathrm{Th} / \mathrm{U}$ atom ratio of 5 . These cross sections were used in several calculations where the Th/U ratio was the variable. The results are shown in Figs. 1.3-1 through 1.3-3.

By using the improved cross-sections, $K_{\text {eff }}$ increased about 10 percent and the IBR decreased a corresponding amount. Therefore, the $T h / U$ ratio was increased to 11 , which was found to yield the desired $\mathrm{K}_{\mathrm{eff}}$ of about 1.10. The associated breeding ratio of this core, based on an equivalent bare homogenized model was found to be 0.48 . The contribution to the breeding ratio fiom the blanket, 
based on previous calculations, was expected to be between 0.3 and 0.4. In order to approximate the upper limit of the blanket breeding ratio, the core neutron leakage was determined, and it was assumed that all these neutrons were captured in the thorium in the blanket. This curve has been included on Fig. 1.3-1 along with the total IBR which is the sum of the blanket and core contributions. A brief list of the parameters for the final reference design are as follows.

(a) Weight per cent oxide in fuel balls - 10 percent

(b) $\mathrm{Th} / \mathrm{U}$ atom ratio $\quad-11$

(c) Average void fraction in the core - 29 percent

(d) Core diameter - $9 \mathrm{ft}$.

In order to compare the breeding ratio for our present design with the value that would be obtained by using ORNL cross-section data for $\mathrm{U}-233$ given in reference (22), curves for IBR and $\mathrm{K}_{\mathrm{eff}}$ based on this data are superimposed on Fig. 1.3-1. It can be seen that this results in an IBR of 1.035 at a $T h / U$ atom ratio of $141 / 2$ where $K_{\text {eff }}$ is 1.10 . This value of the IBR is somewhat higher than the value of .83 obtained by our present calculations. This difference is caused by the values of $\alpha$ which are used and reflects the need of measurements to determine $d$ in the epithermal region.

An alternate to the reference design, which was considered but not investigated thoroughly, was a continuously refueled reactor. Although this concept would involve additional mechanical design problems of loading and unloading devices while the reactor is operating, its compensation would be a higher IBR. It could be designed to have an initial $\mathrm{K}_{\mathrm{eff}}\left(1200^{\circ} \mathrm{F}\right.$, eq. xenon) of slightly above unity and a refueling cycle of between several hours to a week. An exact determination of the required initial reactivity was not made as it is a rather lengthly calculation. A general picture can be gained from Fig. 1.3-1. For a core with the same physical dimensions as the reference design, an atom ratio of about 16 yields a $K_{\text {eff }}$ $\left(1200^{\circ} \mathrm{F}\right.$, eq. xenon) of 1.02 and an IBR of about one. Non-capture leakage will reduce the IBR to about 0.97 . If some of the gaseous fission product xenon diffused out of the fuel elements, a higher $\mathrm{Th} / \mathrm{U}$ ratio could be used with a corresponding higher IBR. The cause of the higher IBR for the continuously fueled core is better neutron economy due to fewer control rod captures.

The remaining parts of this section are concerned with a more detailed analysis of the selected reference design. 


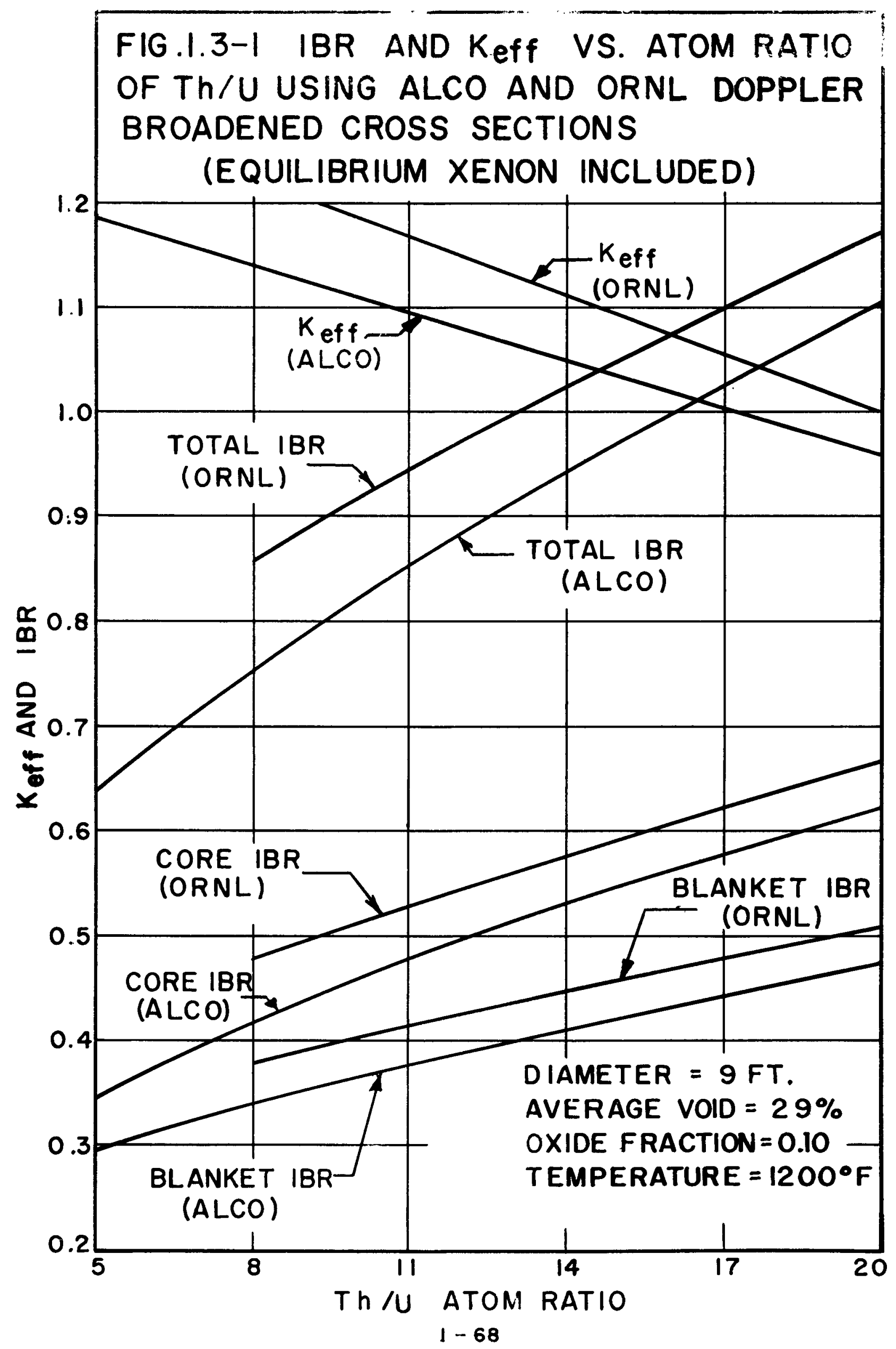


FIG.I.3-2 RESONANCE ESCAPE PROBABILITY P VS. ATOM RATIO Th/U AT $1200^{\circ} \mathrm{F}$ FOR FINAL DESIGN BASED ON A HOMOGENEOUS MODEL.

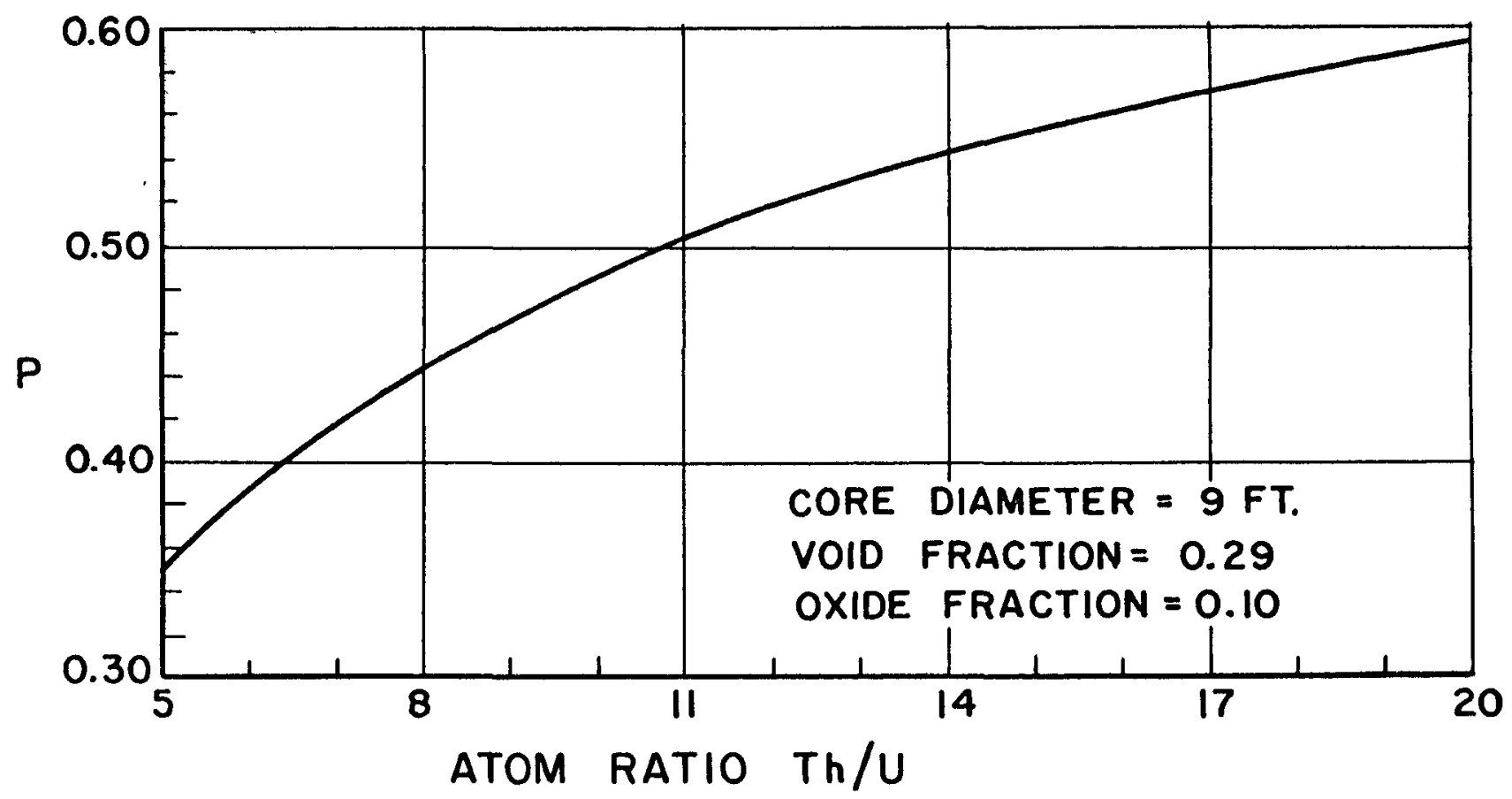

FIG.I.3-3 NEUTRON AGE $\tau$ VS. ATOM RATIO Th/U AT $1200^{\circ} \mathrm{F}$ BASED ON A HOMOGENEOUS MODEL

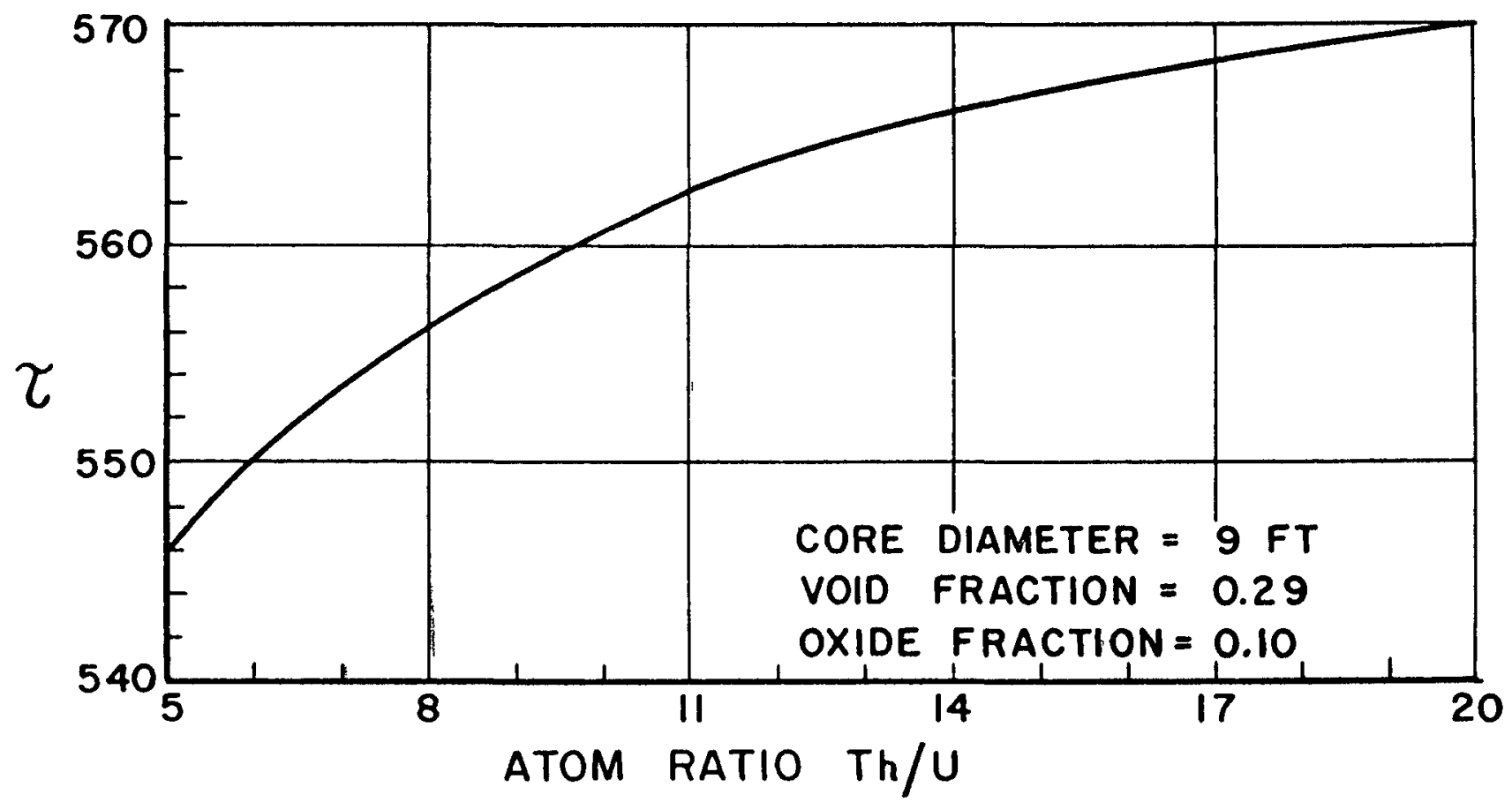




\subsection{Analysis of Final Core}

With the selection of the final design, the large number of variables considered previously has been significantly reduced. It is now possible to develop a mechanical design of the core so that succeeding calculations will be based on a more precise geometric model. Once the geometry is fixed, multi-region calculations can be made to determine the core parameters more accurately than the homogenized core model used in the survey. This section is concerned primarily with the detailed analysis of the final core design. A summary of the final reactor characteristics is listed in Table 1.4-3 in Section 1.4.9.

\subsubsection{Core Loading}

As discussed in Section 1.3, the necessary atom ratio of $T h / U$ to yield a desired $K_{\text {eff }}$ of 1.10 (Figure $1.3-1$ ) was found to be 11 to 1 . This corresponds to an initial loading of $90.2 \mathrm{~kg} \mathrm{U}-233$ and 992.2 $\mathrm{kg}$ Th-232 in the core. This loading results in about $30 \mathrm{~kg}$ of U-233 above the critical mass.

Referring to Figure 1.3-1, it is seen that the atom ratio necessary to make the reactor critical with equilibrium Xenon is about 16 to 1 resulting in a loading of $63.7 \mathrm{~kg}$ of $U-233$. This ratio is of interest for a reactor which is to be refueled continuously. This type of reactor operation would increase the IBR by about 0.15 due to lower neutron losses to the control rods. The same effect could be achieved by using fertile material in the control rods and thus convert and control at the same time.

\subsubsection{Control Rod Requirements}

The number of control rods required was determined by initially calculating the reactivity worth of a centerline control rod using the method outlined in references (13), (14). The worth of an off-center 
rod was found by weighing the centerline rod worth by a $\mathrm{J}_{\mathrm{o}}$ Bessel function squared. The calculation of the centerline rod worth is based on the rod being black to thermal neutrons. Since the rods are to be hollow, the fast neutron properties of the rod are the same as those of a void. Fig. 1.4-1 shows the worth of the centerline rod as a function of rod radius. From both nuclear and mechanical design considerations a four inch diameter control rod is acceptable. Larger rods with their incre: sed weight present a mechanical problem in designing a decelerating mechanism while smaller rods control less reactivity.

By using four inch diameter rods, and locating twelve of them in a ring of two foot radius, the total worth of the rods is approximately 17.3 percent in reactivity. This worth is conservative because the rods are placed in a graphite ring which causes the thermal flux to peak as opposed to the regular Bessel function flux distribution that was assurned. Actually, the thermal flux is higher in the graphite ring than at the core centerline as shown in Fig. 1.4-3.

Europium and Haynes $-25 *$ were both considered for control rod material. Europium is blacker to the thermal neutrons for the same thickness, but it is considerably more expensive. Haynes -25 has a high cobalt content but the associated activity is not expected to cause any difficulty. Therefore, the reduced cost of Haynes -25 led to its selection as the control rod material.

\subsubsection{Power and Flux Distributions}

In order to determine $\mathrm{K}_{\text {eff }}$ and the initial breeding ratio more accurately than the homogenized model it was necessary to utilize a multiregion calculation model. The modified two group theory was used employing group constants as found by the methods as outlined in Section 1.2.1.

* Chemical composition of Haynes-25: Cr 19-21\%, Co 46-53\%, W-14-16\%, Ni 9-11\%, Fe 3\%, Si 1\%, Mn 1-2\%, C 0.05-0.15\%, $\mathrm{P} 0.04 \%$ and $\mathrm{S} 0.03 \%$. 
FIC.1.4-1 REACTIVITY (\%) OF A SINGLE COIVTROL ROD VS. RADIUS OF ROD (INCHES)

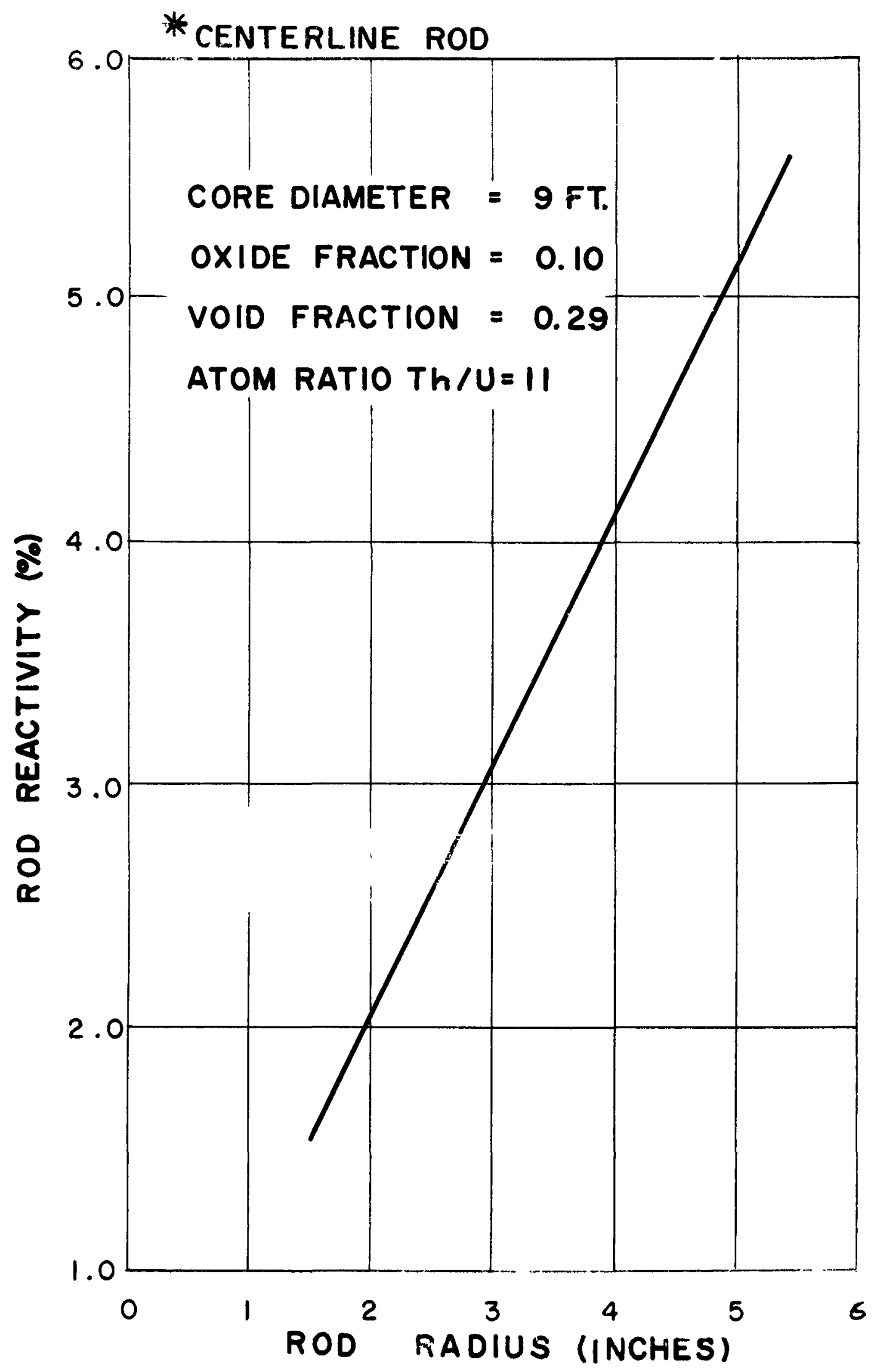




\subsubsection{Radial Flux and Power}

The radial flux and power were calculated using a one dimensional machine code (15). It was necessary to homogenize the graphite webs (between the outer six fuel pockets) and the fuel into an equivalent annular fuel region. The graphite ring which houses the control rods and a two inch graphite ring between core and blanket was preserved. Figure 1.4-2 shows the radial geometry used along with the materials considered. The results of the calculations are shown in Figures 1.4-3 and 1.4-4, where the flux and power are plotted as a function of radius.

The control rods reduce the peaking of the radial flux in the graphite regions and flatten the radial power distribution in the rodded region of the core adjacent to the graphite. Therefore, the power distribution with the rods inserted was determined. The worth of the rods fully inserted was known; thus, the subcriticality of the reactor was known. An equivalent poison was added to the graphite ring that houses the rods until the $K_{\text {eff }}$ of the reactor equaled that due to full insertion of the rods. Figure (1.4-5) illustrates the effect on $\mathrm{K}_{\mathrm{eff}}$ of increasing $\boldsymbol{\Sigma}_{\mathrm{a}}$ in the graphite ring. When the proper $\boldsymbol{\Sigma}$ a was determined, corresponding to $\mathrm{K}_{\mathrm{eff}}$ equal to approximately 0.93 , the power and flux distribution was recalculated. The former is shown in Figure 1.4-6.

\subsubsection{Axial Flux and Power}

The fuel pockets extend the full length of the core in the axial direction; therefore, to compute the average axial flux and power distribution the core region must be homogenized.

The partially inserted control rods were replaced by an equivalent $\Sigma_{p}$ in the region into which the rods are inserted (16). In this type of calculation, the $K_{\text {eff }}$ of the core with the rods fully inserted is determined by:

$$
\mathrm{K}_{\mathrm{eff}}(\text { rods in })=\frac{1}{1-(\rho \text { reactor }-\rho \text { rods })}
$$


FIG.I.4-2 SCHEMATIC OF RADIAL CONFIGURATION USED IN MULTIREGION CALCULATION (DIMENSIONS IN INCHES)

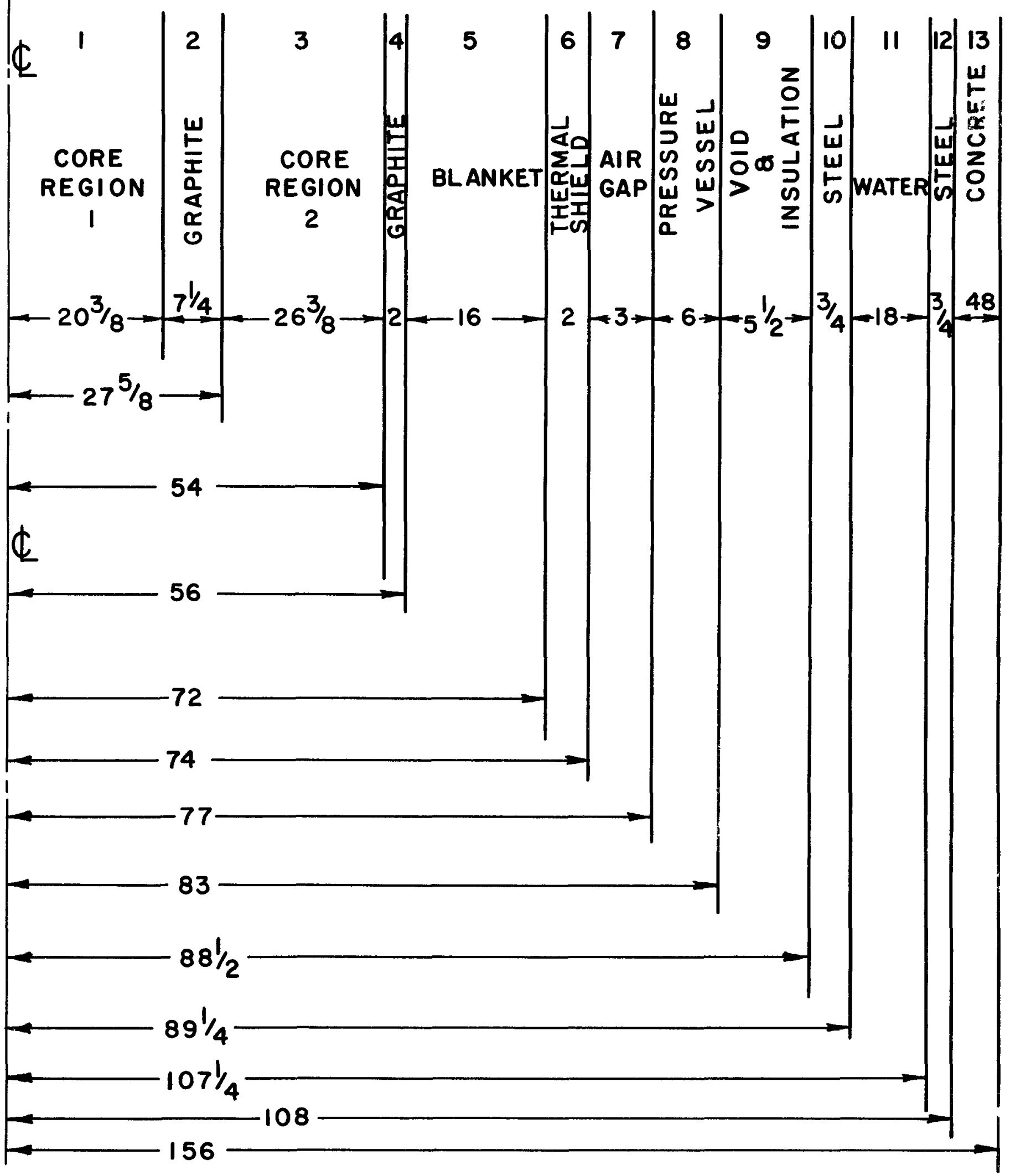


FIG. 1.4-3 RELATIVE * RADIAL FAST AND THERMAL FLUX FOR FINAL DESIGN AT $1200^{\circ} \mathrm{F}$.

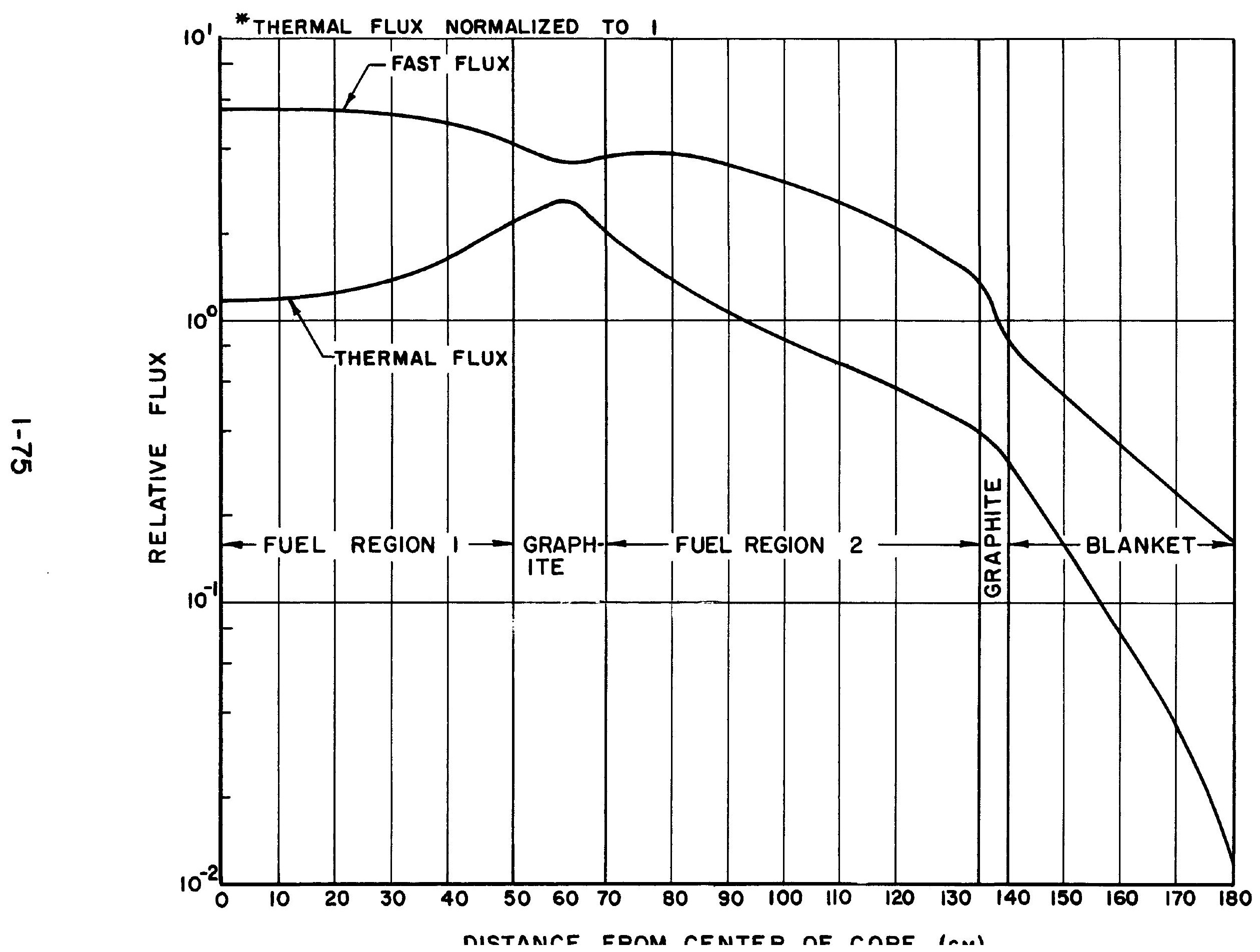


FIG.1.4-4 RELATIVE * RADIAL POWER FOR FINAL DESIGN AT $1200^{\circ} \mathrm{F}$. (RODS FULLY WITHDRAWN)

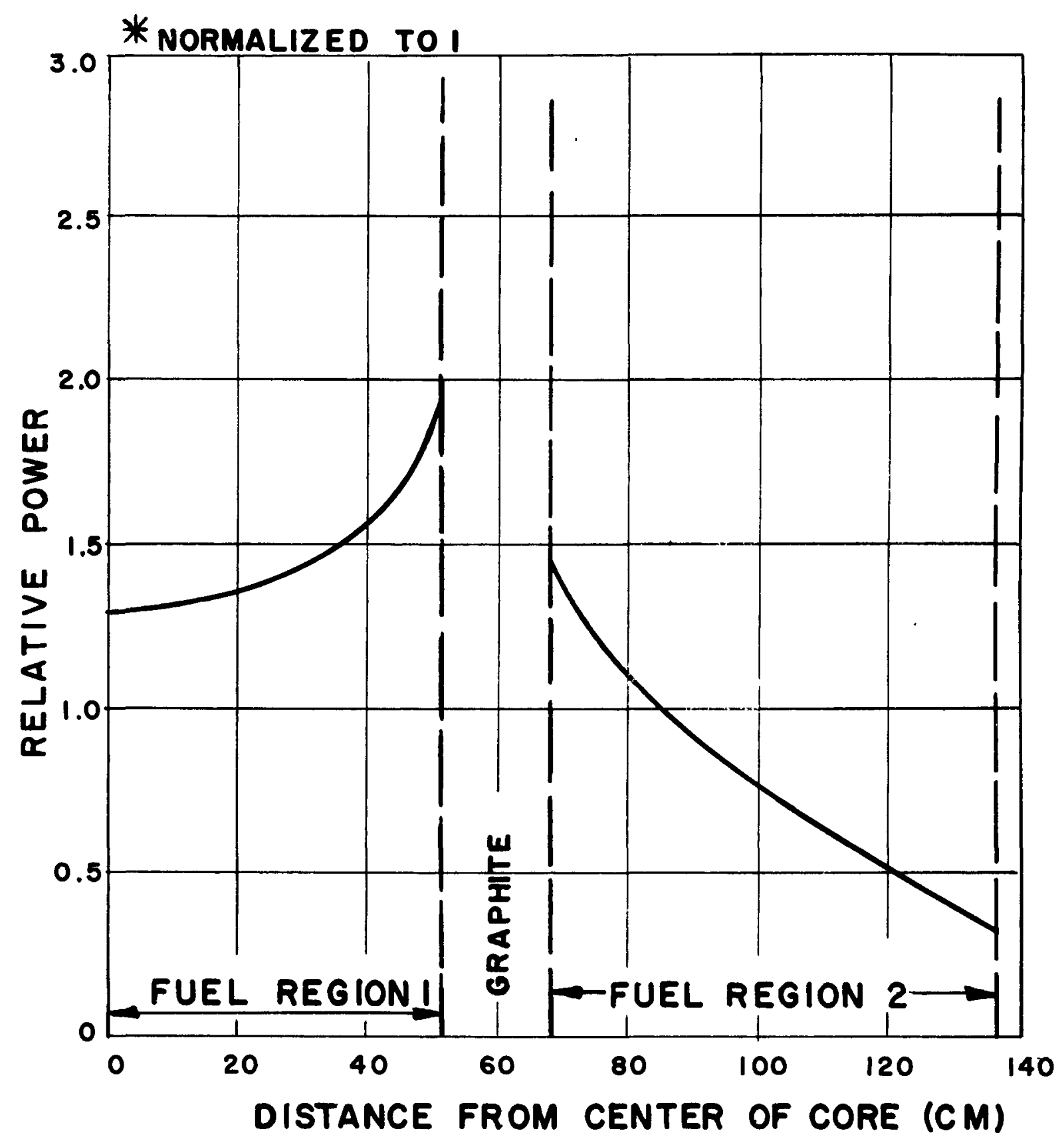


FIG. I.4-5 EFFECT OF INCREASING $\Sigma_{p}$ IN GRAPHITE RING ON KEFF $\left(1200^{\circ} \mathrm{F}\right)$

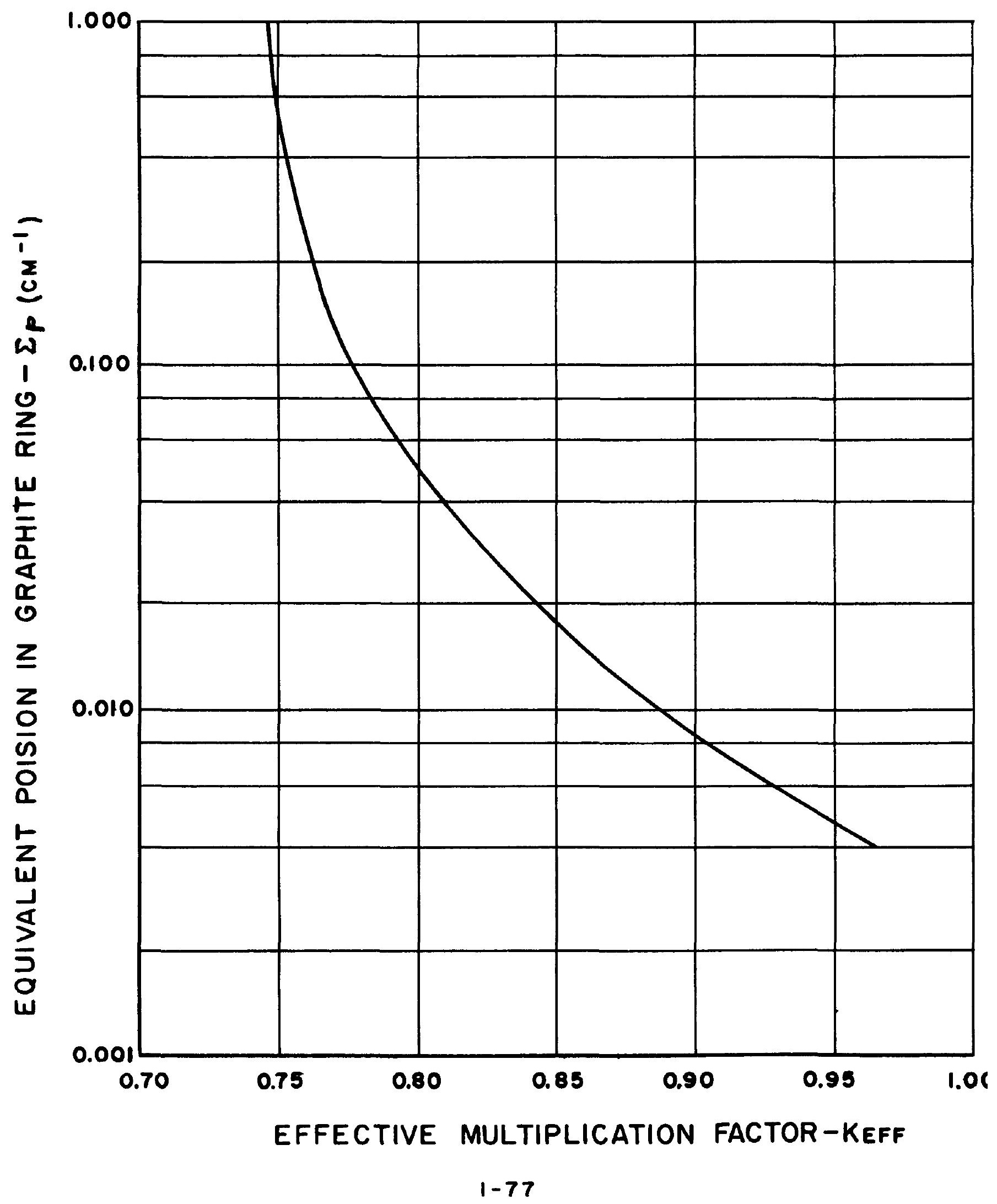


FIG. I.4-6 RELATIVE *RADIAL POWER FOR

FINAL DESIGN AT $1200^{\circ} \mathrm{F}$.

(RODS FULLY INSERTED)

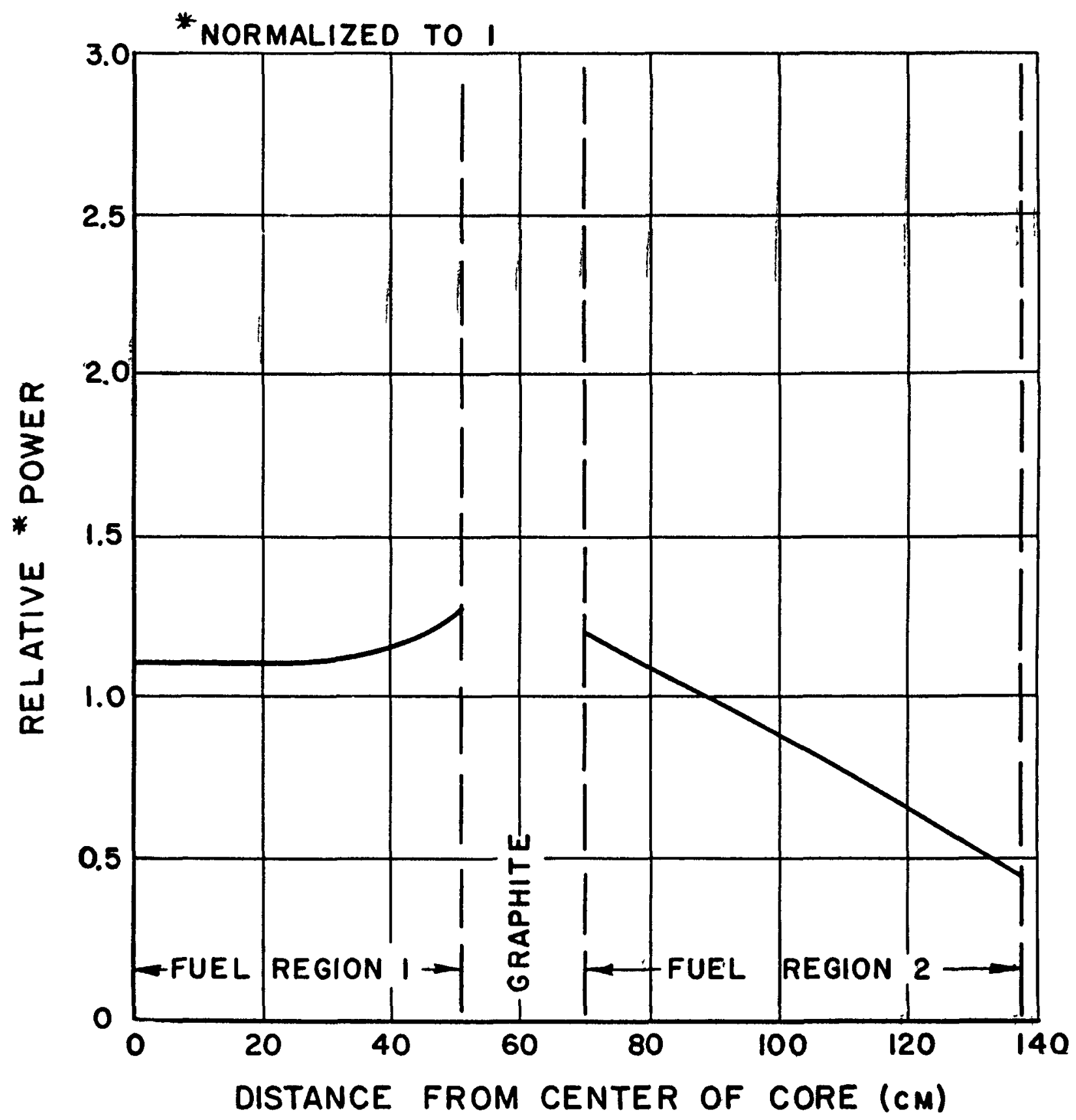


Then an equivalent thermal poison is determined that will reduce $K_{e f f}$ to $K_{\text {eff }}(\operatorname{rods}$ in).

$$
\sum_{a}^{T^{\prime}}=\frac{V \sum_{f \text { th }}}{\frac{\left(1+T B^{2}\right) K_{\text {eff }}(\operatorname{rods} \text { in })}{P}-\left(\frac{1-P}{P}\right) K_{f}}-D_{s} B^{2}(1.4-2)
$$

where

$$
\sum_{p}=\sum_{a}^{T^{\prime}}-\sum_{a}^{T}(\text { core })
$$

The core is then considered as two regions, one containing $\Sigma_{p}$ in excess of $\sum_{a}^{T}$ and the other region containing only $\sum_{a}^{T}$. An iterative calculation is then made to determine the location of the interface of the two regions so that $K_{e f f}$ is just unity. The interface is then taken as the depth of the control rod insertion.

The results of these calculations are shown in Figures 1.4-7 and 1.4-8 as flux and power distributions as a function of distance from the top of the core.

\subsubsection{Initial Breeding Ratio.}

The initial breeding ratio in the nuclear parameter survey was calculated based on an homogenized core. After a reference design was selected, it was possible to obtain a more exact value of the IBR using multi-region flux distributions. There is a breeding contribution in both fuel regions and from the blanket. The overall IBR may be defined as:

$$
\text { IBR }=\frac{H_{1} V_{1}+H_{2} V_{2}+H_{B} V_{B}}{J_{1} V_{1}+J_{2} V_{2}+J_{B} V_{E}}=\frac{\sum_{i} H_{l} V_{l}}{\sum_{i} J_{i} V_{l}}
$$

where: 1 = designates the region ( 1 = fuel region one;

$$
2 \text { = fuel region two, } B=\text { blanket) }
$$

$$
\begin{aligned}
& H_{i}=N_{i}^{02}\left[\bar{\phi}_{t h i} \sigma_{a t h i}^{02}+\bar{\phi}_{f i} \bar{\sigma}_{a f i}^{02}\right] \\
& J_{i}=N_{i}^{23}\left[\bar{\phi}_{t h i} \sigma_{a t h i}^{23}+\bar{\phi}_{f i} \bar{\sigma}_{a f i}^{23}\right]
\end{aligned}
$$


FI. 1, 4-7 RELATIVE AXIAL FAST AND THERMAL FLUX FOR FINAL DESIGN AT $1200^{\circ} \mathrm{F}$ AT START OF CORE LIFE. (RODS IN CRITICAL POSITION)

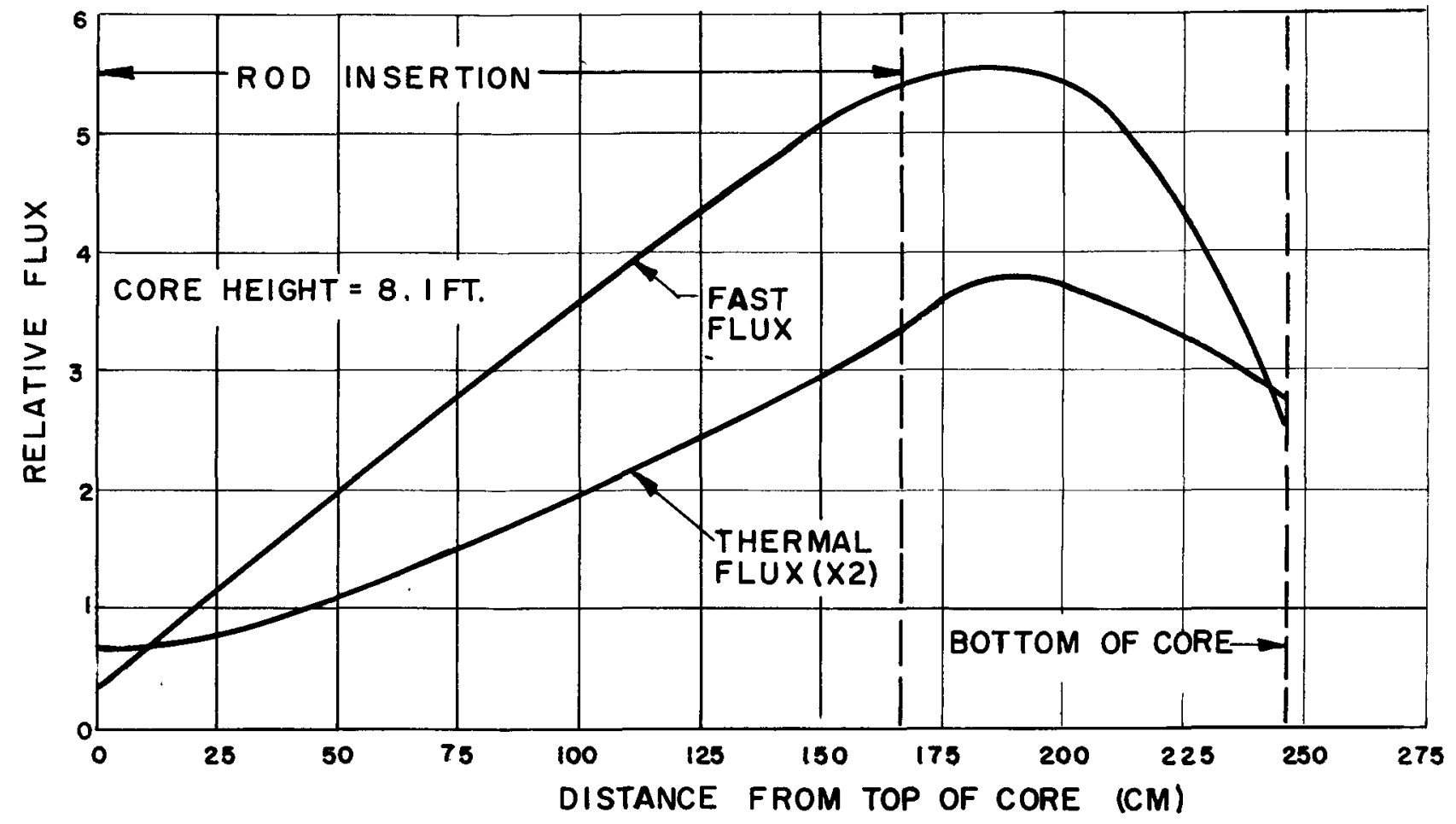

FIG.I.4-8 RELATIVE AXIAL POWER DISTRIBUTION AT $1200^{\circ} \mathrm{F}$ WITH EQUILIBRIUM XENON.(NO FISSION PRODUCTS)

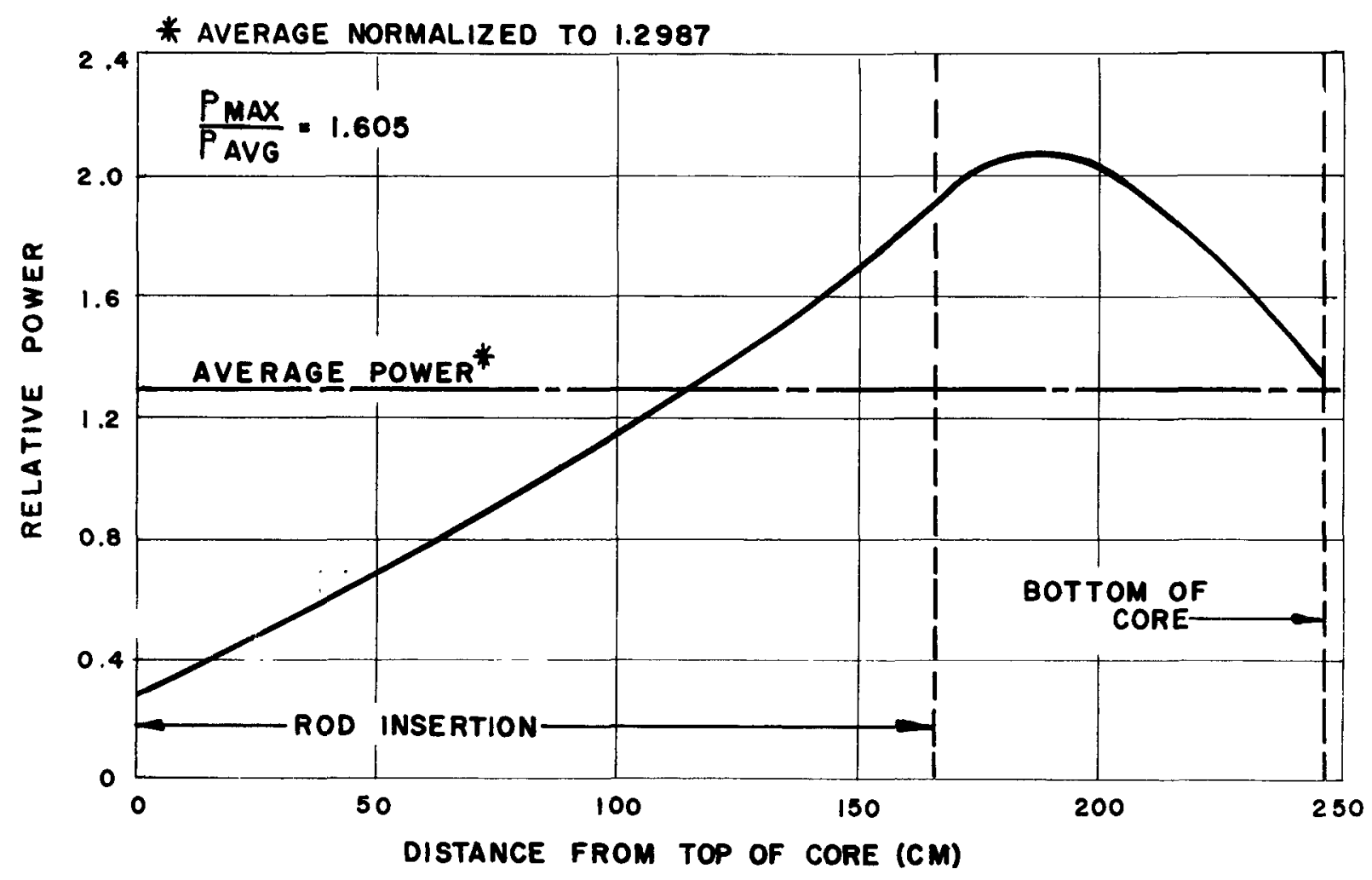


The thermal cross-sections were taken at $1200^{\circ} \mathrm{F}$ and the average fast cross-sections were obtained by the method described in Section 1.2.2. The average relative fluxes in each region were obtained by integrating the multi-region flux plots as given in Section 1.4.3. The actual values of the thermal fluxes in the fuel regions were obtained from:

$$
\phi_{\operatorname{th}(1,2)}=\frac{\left(F \cdot P_{.}\right)_{1,2} c}{V_{i}\left[N_{1,2}^{23} \sigma_{f t h i}^{23}+\left(\frac{\Phi_{f}}{\Phi_{t h}}\right) \sigma_{f f i}^{23} N_{1,2}^{23}\right]}
$$

where: $(F . P .)_{1,2}=$ Fractional Power in the appropriate region $\mathrm{c}=3.12133 \times 10^{16}$ (fissions $/ \mathrm{MW}-\mathrm{sec}$ )

The fractional power for regions one and two were obtained by integration of the radial multi-region power plots in Sections 1.4.3. The value of $\bar{\phi}_{f} / \bar{\phi}_{t h}$ is the ratio of the average relative fluxes.

Table $1.4-1$ is a tabulation of the parameters and results of the preceding calculations. It is seen that the IBR due to the core is 0.477 and the IBR due to the blanket is 0.353 yielding an overall initial conversion ratio of 0.83 . It is to be noted that the IBR due. to the core alone is quite close to the value of 0.478 as determined by the homogeneous model.

The IBR of the blanket includes that portion of blanket which extends radially above and below the core. The IBR contribution from the portion of the blanket which is adjacent to the core is 0.315 . The rest of the blanket was assumed to be in a flux level of $25 \%$ of the adjacent blanket and contributes only 0.038 to the IBR. 
TABLE 1.4-1

CALCULATION OF INITIAL BREEDING RATIO

\begin{tabular}{|c|c|c|c|}
\hline Parameter & Region One & Region Two & Blanket \\
\hline$\phi_{\mathrm{th}} \times 10^{14}\left(\mathrm{n} / \mathrm{cm}^{2}-\mathrm{sec}.\right)$ & 2.298 & 1.608 & $0.259 *$ \\
\hline$\phi_{f} \times 10^{14}\left(\mathrm{n} / \mathrm{cm}^{2}-\mathrm{sec}.\right)$ & 7.629 & 4.938 & 0.654 \\
\hline Power (MW) & 87.16 & 262.84 & $\ldots$ \\
\hline $\mathrm{N}^{02} \times 10^{-20}\left(\mathrm{at} / \mathrm{cm}^{3}\right)$ & 2.375 & 1.934 & 20.301 \\
\hline $\mathrm{N}^{23} \times 10^{-20}\left(\mathrm{at} / \mathrm{cm}^{3}\right)$ & 0.215 & 0.175 & $\cdots \cdots$ \\
\hline $\mathrm{N}^{0} \times 10^{-20}\left(\mathrm{at} / \mathrm{cm}^{3}\right)$ & 5.179 & 4.218 & 40.602 \\
\hline $\mathrm{N}^{\mathrm{c}} \times 10^{-22}\left(\mathrm{at} / \mathrm{cm}^{3}\right)$ & 5.129 & 5.763 & 4.462 \\
\hline Temperature $\left({ }^{\circ} \mathrm{F}\right)$ & 1200 & 1200 & 800 \\
\hline IBR (contribution) & 0.124 & 0.353 & 0.353 \\
\hline
\end{tabular}

* Since the thermal flux increases by about a factor of 2 during the life of the core and because the blanket is not changed for several core lifetimes, the average thermal flux in the blanket was based on that which occurs at the mid-life of the core. 


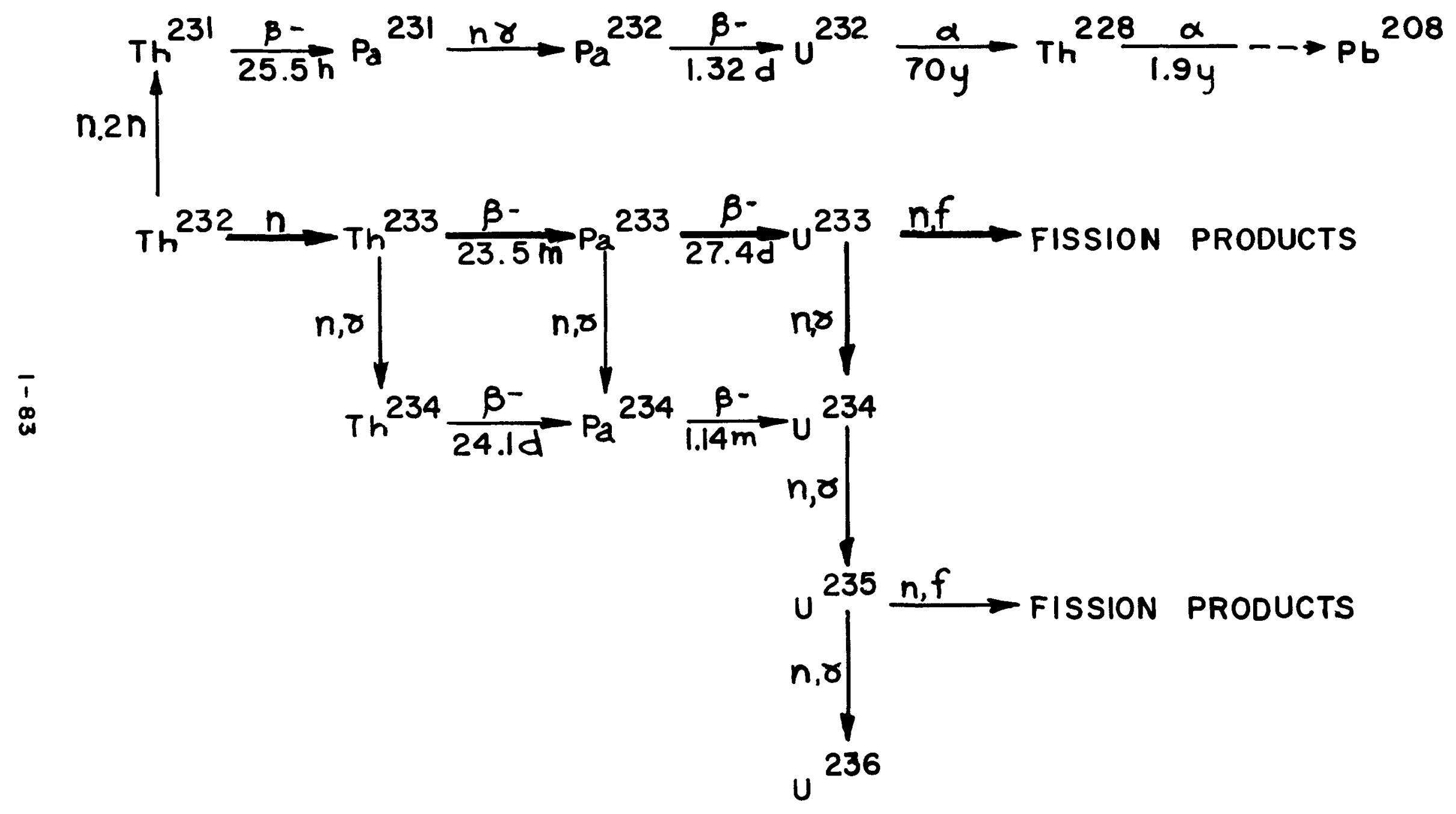

$\frac{\pi}{0}$

THORIUM - URANIUM ABSORPTION DECAY SCHEME 


\subsubsection{Buildup of Isotopes}

The thorium-uranium absorption decay scheme is shown in Figure 1.4-9. The most important chain commences with the absorption of neutrons by $\mathrm{Th}-232$ to produce $\mathrm{Th}-233$ which in turn decays to $\mathrm{Pa}-233$ by $\beta^{-}$emission with a half life of $23.5 \mathrm{~m}$; Pa-233 $\beta^{-}$decays to U-233 with a half life of $27.4 \mathrm{~d}$. The primary reaction in $\mathrm{U}-233$ is the absorption of neutrons to produce fissioning and the associated fission products. Higher isotopes of uranium produce nearly as many neutrons as they absorb and therefore can be neglected (18).

This chain and the differential equations describing it may be used for both the core and blanket. The general differential buildup equations for the principal isotopes $T h-232^{\prime}\left(\mathrm{N}^{02}\right), T h-233\left(\mathrm{~N}^{03}\right)$ and $\mathrm{Pa}-233\left(\mathrm{~N}^{13}\right)$ and $\mathrm{U}-233\left(\mathrm{~N}^{23}\right)$ may be expressed by:

$$
\begin{aligned}
d N^{02} / d t= & -\sigma_{a t h}^{02} \bar{\phi}_{t h} N^{02}-\bar{\sigma}_{a f}^{02} \bar{\phi}_{f} N^{02} \\
d N^{03} / d t= & \sigma_{a t h}^{02} \bar{\phi}_{t h} N^{02}+\bar{\sigma}_{a f}^{02} \bar{\phi}_{f} N^{02} \\
& -\lambda^{03} N^{03}-\sigma_{a t h}^{03} \bar{\phi}_{t h} N^{03} \\
d N^{13} / d t= & \lambda^{03} N^{03}-\lambda^{13} N^{13}-\bar{\sigma}_{a t h}^{13} \bar{\phi}_{t h} N^{13} \quad(1.4-8) \\
d N^{23} / d t= & \lambda^{13} N^{13}-\sigma_{a t h}^{23} \bar{\phi}_{t h} N^{23}-\bar{\sigma}_{a f}^{23} \bar{\phi}_{f} N^{23}(1.4-10)
\end{aligned}
$$

where:

$$
\begin{aligned}
& \lambda^{13}=\text { decay constant of } \mathrm{Pa}-233=2.9273 \times 10^{-7} \mathrm{sec}^{-1} \\
& \lambda^{03}=\text { decay constant of } \mathrm{Th}-233=4.9570 \times 10^{-1} \mathrm{sec}^{-1}
\end{aligned}
$$

For convenience of calculation the following quantities were defined:

$$
a=\sigma_{a t h}{ }^{23} \Phi_{t h}+\bar{\sigma}_{a f}^{23} \bar{\phi}_{f}
$$




$$
\begin{aligned}
& b=\lambda^{13}+\sigma_{a t h}^{13} \bar{\phi}_{t h} \\
& d=\sigma_{a t h}^{02} \bar{\phi}_{t h}+\bar{\sigma}_{a f}^{02} \bar{\phi}_{f} \\
& e=\lambda^{03}+\sigma_{a t h}^{03} \bar{\phi}_{t h}
\end{aligned}
$$

Therefore, the general differential buildup equations may be written as:

$$
\begin{aligned}
& d N^{01} / d t=-d N^{02} \\
& d N^{03} / d t=d N^{02}-e N^{03} \\
& d N^{13} / d t=\lambda^{03} N^{03}-b N^{13} \\
& d N^{23} / d t=\lambda^{13} N^{13}-a N^{23}
\end{aligned}
$$

\subsubsection{Buildup in the Core}

For these calculations, the core was homogenized into one region and the graphite and oxygen concentration were assumed to remain constant.

The general buildup equations may be simplified since the concentration of $\mathrm{Th}-232$ remains essentially constant over time intervals of a few months, changing by less than $1 \%$ during 360 days of reactor operation; in addition, Th- 233 will attain its equilibrium concentration in less than a day of reactor operation so its equilibrium value may be used for all times after startup without introducing any appreciable error. The initial conditions may be expressed by:

$$
N^{02}(t=0)=N_{0}^{02}
$$




$$
\begin{aligned}
& N^{03}(t=0)=N^{13}(t=0)=0 \\
& N^{23}(t=0)=N_{0}^{23}
\end{aligned}
$$

With the above assumptions and initial conditions, the integrated buildup equations become:

$$
\begin{aligned}
& N^{02}(t) \cong N_{0}^{02} \\
& N^{03}(t) \cong \frac{d}{e} N_{0}^{02} \\
& N^{13}(t) \cong \frac{\lambda^{03} d N_{0}^{02}}{e b}\left(1-e^{-b t}\right)+N_{0}^{13} e^{-b t} \\
& N^{23}(t) \cong \frac{\lambda^{13}}{b-a}\left[N_{0}^{13} \frac{\lambda^{03} d N_{0}^{02}}{e b}\right]\left(e^{-a t}-e^{-b t}\right)+ \\
&+\frac{\lambda^{13}}{a}\left(\frac{\lambda^{03} d N_{0}^{12}}{e b}\right)\left(1-e^{-a t}\right)+N_{0}^{23} e^{-a t}
\end{aligned}
$$

The buildup equations depend upon the average fast and thermal fluxes; the latter, however changes with time due to the constantly changing core composition. In an attempt to take this into account, the average fluxes to be used during the first 60 days of buildup were recalculated from the atom densities at the start of core life. From these values, the atom densities at the end of 60 days were recalculated by the buildup equations. The new atom densities were then used to calculate the average fluxes to be used during the next 60 days of isotope buildup. This method was repeated through 120 days of reactor operation. Figure 1.4-10 shows the change in the average fluxes as a function of the days of continuous reactor operation. Figure 1.4-11 shows the buildup of the isotopes in the core as a function of the days of continuous reactor operation. These results assume an average operating temperature of $1200^{\circ} \mathrm{F}$ and a power level of $350 \mathrm{MW}$.

\subsubsection{Buildup in the Blanket}

As in the core, the concentration of Th-232 in the blanket may be considered constant and the equilibrium concentration of Th-233 may be 

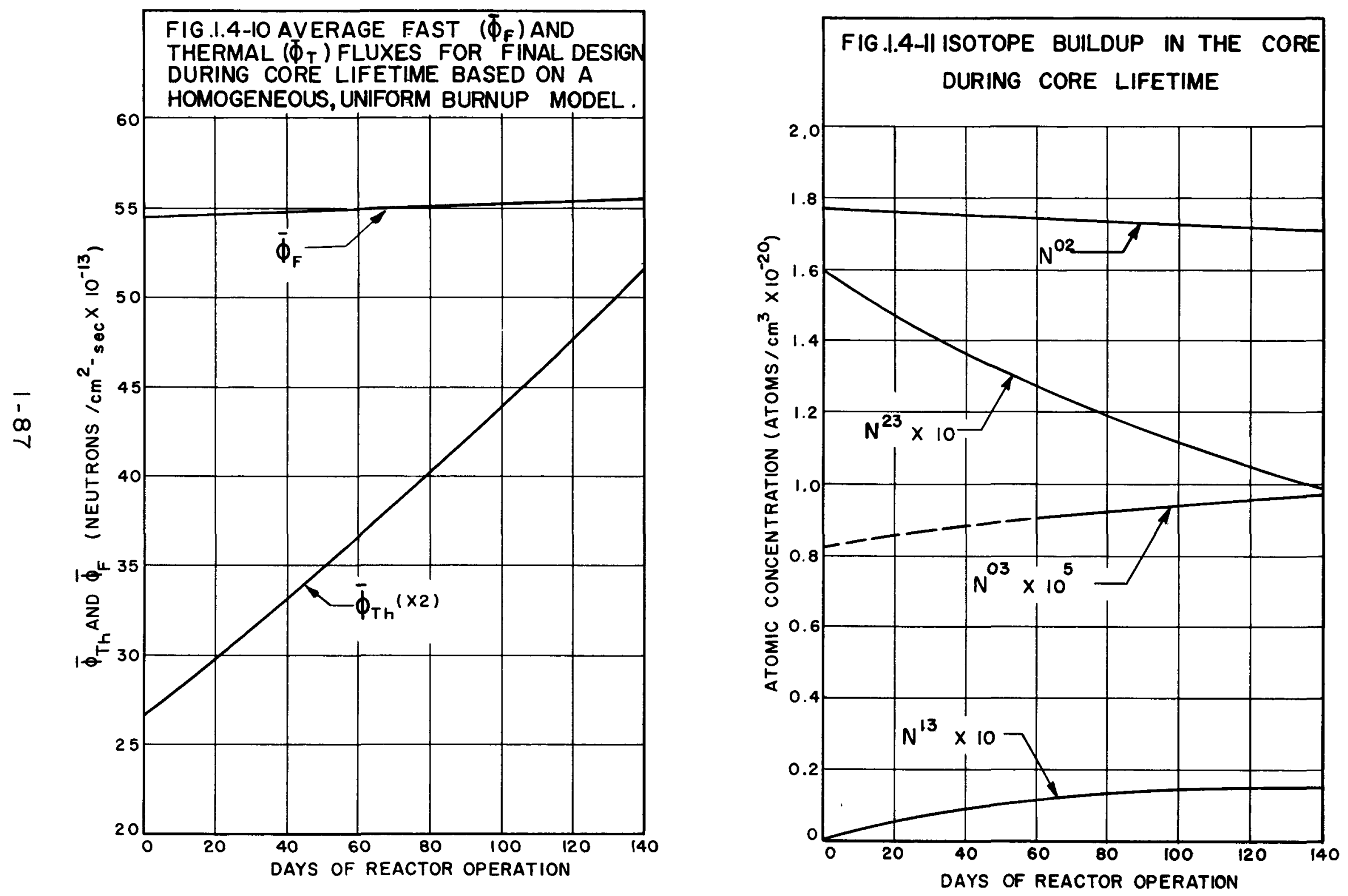
used any time after the start of blanket lifetime. In addition, the average fast and thermal fluxes in the blanket were assumed constant with time because the loading cycle in the blanket is considerably longer than that of the core. The average values of the fluxes were taken at the midlife of the core. The initial conditions may be expressed by:

$$
\begin{aligned}
& N_{0}^{02}=20.3010 \times 10^{20} \text { atoms } / \mathrm{cm}^{3} \\
& N_{0}^{23}=N_{0}^{03}=N_{0}^{13}=0
\end{aligned}
$$

The integrated buildup equations for the blanket become:

$$
\begin{aligned}
N^{02} \cong N_{0}^{02} \\
N^{03} \cong \frac{d}{e} N_{0}^{02} \\
N^{13} \cong \frac{\lambda^{03} d N_{0}^{02}}{b e}\left(1-e^{-b t}\right) \\
N^{23} \cong \frac{\lambda^{13}}{b-a}\left[\frac{\lambda^{03} d N_{0}^{02}}{e t s}\right]\left(e^{-b t}-e^{-a t}\right)+ \\
+\frac{\lambda^{13}}{a}\left(\frac{\lambda^{03} d N_{0}^{02}}{e b}\right)\left(1-e^{-a t}\right)
\end{aligned}
$$

The buildup of U-233 and $\mathrm{Pa}-233$ in atoms per cc of blanket volume as a function of days of continuous full power operation are plotted in Figure 1.4-12.

The blanket loading will not be changed as often as the core in order to reduce the the reprocessing and refabrication charges for the blanket as discussed in Section 3.0. The blanket lifetime affects the heat generation rate in the blanket and also influences the reactivity since the production of U-233 in the blanket adds reactivity which partially offsets the loss in reactivity from burnup of U-233 in the core. The ratio of heat produced in the blanket due to fission of U-233 to that in the core may be written as:

$$
\gamma=\frac{V\left(\sigma_{f B}^{23} \bar{\phi}_{t h B}+\bar{\sigma}_{f f B} \bar{\phi}_{f B}\right) N^{23}}{350 c}
$$




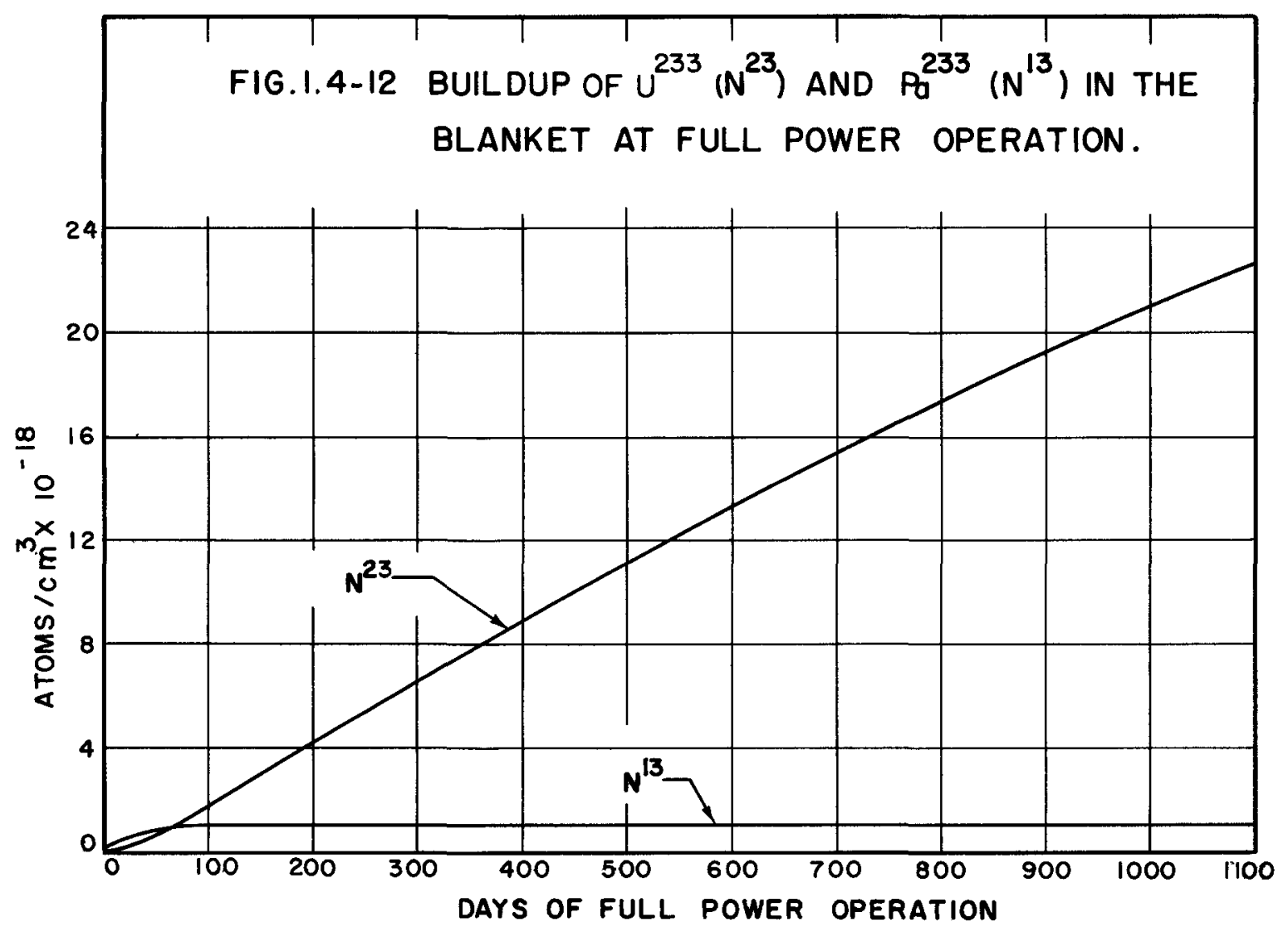

FIG . I. 4-I3 RATIO OF HEAT PRODUCED IN BLANKET TO CORE AT FULL POWER OPERATION.

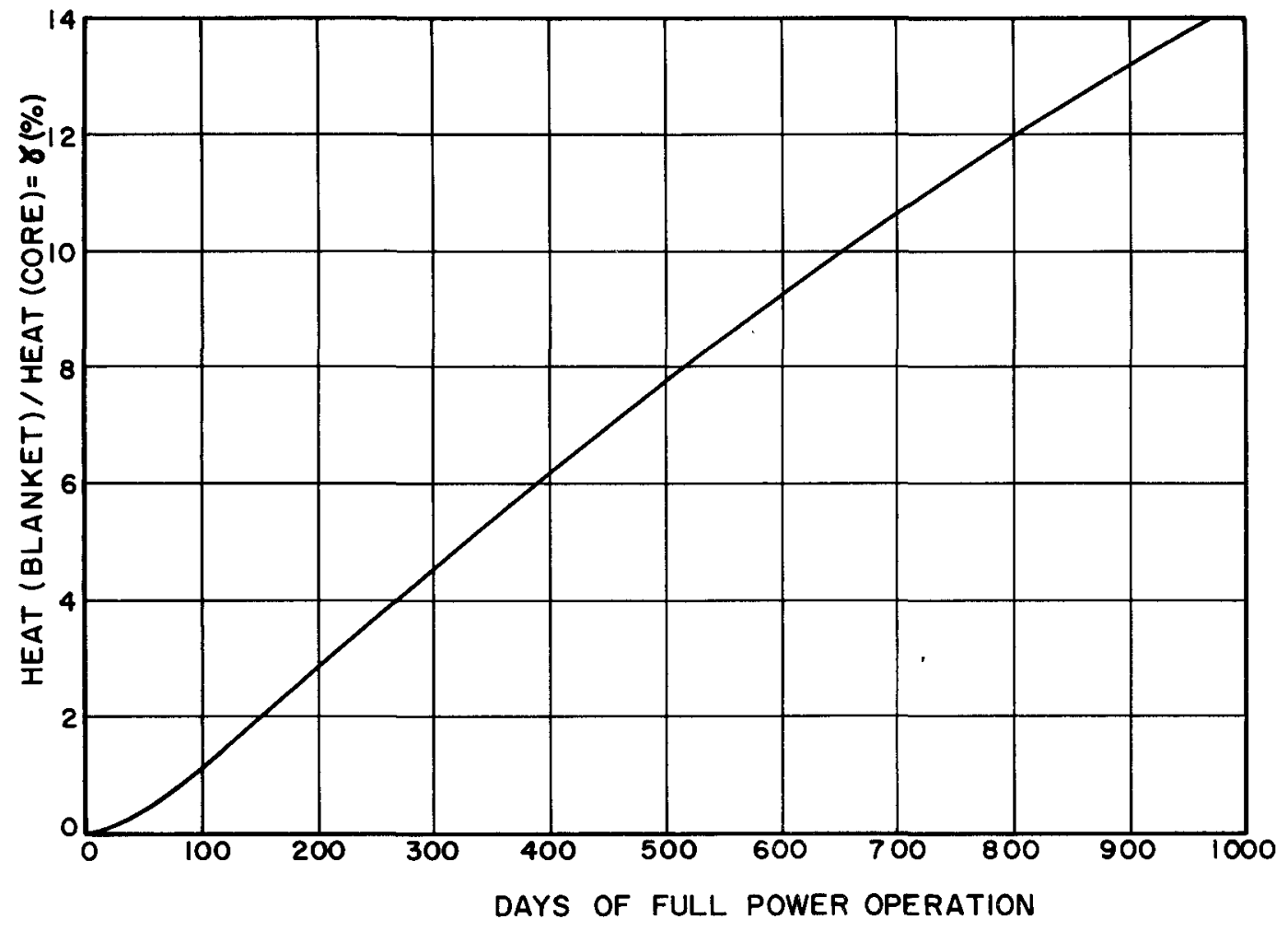


where:

$$
\begin{aligned}
& \mathrm{V}=\text { Blanket volume }=10.25 \times 10^{6} \mathrm{cc} \\
& \mathrm{C}=3.1213 \times 10^{16} \mathrm{fissions} / \mathrm{MW}-\mathrm{sec} \\
& \left(\bar{\phi}_{\mathrm{fB}} \bar{\sigma}_{\mathrm{ff}}+\bar{\phi}_{\mathrm{thB}} \bar{\sigma}_{\mathrm{fth}}\right)=49.3898 \times 10^{-10_{\text {fissions }} / \mathrm{sec} \text {-atom }}
\end{aligned}
$$

The fraction $\gamma$, expressed in percent, is plotted as a function of blanket exposure time in Figure 1.4-13. As discussed in Section 3.0, 1/10 of the blanket will be changed with each core reloading. After reaching equilibrium the heat generation is equivalent to a single blanket lifetime of 550 days which results in a value of $\gamma=8.5 \%$.

\subsubsection{Core Lifetime}

The core lifetime is dependent on the initial loading of $\mathrm{U}-233$, the buildup of U-233 from captures in $\mathrm{T}$ in-232, and the buildup of fission products.

Due to the relatively high power at which the reactor will operate, about $14 \mathrm{~kg}$ of U-233 will be consumed per month. However, due to a breeding ratio of about 0.48 in the core, a large amount of fuel is being formed as the reactor operates which increases the core lifetime.

In Section 1.4.5.1, the buildup of Th-232, Th-233, Pa-233, and $\mathrm{U}-233$ in the core was obtained. It was mentioned that the average fluxes for each 60 days of operation were obtained from the atom densities present at the start of each 60 day period. In the calculation of these fluxes, all of the fast and thermal parameters, $K_{e f f}$, and breeding ratio were also obtained at the end of each 60 day period. The calculation of $\mathrm{K}_{\mathrm{eff}}$ included equilibrium xenon and the buildup of fission products in the fuel balls; the latter was included using a method described in reference (17). This reference contains a curve specifying the amount of natural boron that yields a thermal absorption crosssection which is equivalent to the fission product absorption for a given amount of fuel burnup. The curve is based on U-235 as the fuel; since the fission product absorption in $U-233$ is about $10 \%$ less than $U-235$, the poisoning was reduced by this amount.

Table 1.4-2 is a tabulation of the results of the core lifetime cal-

culations. Figure $1.4-14$ is a plot of $K_{\text {eff }}$ and breeding ratio as a function of days of rcactor operation. It is seen that the rate at which $\mathrm{K}_{\text {eff }} \mathrm{drops}$ 
TABLE $1.4-2$

CORE LIFETIME TABLE

\begin{tabular}{|c|c|c|c|}
\hline Property & Initial & $\mathrm{t}=60$ (days; & $\mathrm{t}=120\langle$ days $\rangle$ \\
\hline $\mathrm{N}^{02}(\mathrm{~kg})$ & 992.24 & 979.23 & 965.51 \\
\hline $\mathrm{N}^{23}(\mathrm{~kg})$ & 90.19 & 71.94 & 59.11 \\
\hline $\mathrm{D}_{\mathrm{f}} \mathrm{cm}$ & 1.6628 & 1.6594 & 1.6569 \\
\hline$P$ & 0.5036 & 0.5481 & 0.5825 \\
\hline$T \mathrm{~cm}^{2}$ & 562.40 & 567.29 & 571.04 \\
\hline$\Sigma_{\text {at }}$ (total) $\mathrm{cm}^{-1}$ & 0.004881 & 0.004180 & 0.003620 \\
\hline $\mathrm{D}_{\mathrm{S}} \mathrm{cm}$ & 1.1369 & 1.1378 & 1. 1385 \\
\hline Core Breeding Ratio & 0.474 & 0.511 & 0.547 \\
\hline$K_{\text {eff }}$ & 1.1001 & 1.0373 & 0.9860 \\
\hline $\mathrm{N}\left(\right.$ Boron) $\times 10^{-17}$ atoms $/ \mathrm{cm}^{3}$ & 0 & 3.3766 & 4.6119 \\
\hline $\mathrm{N}$ (Xenon) $\times 10^{-16}$ atoms $/ \mathrm{cm}^{3}$ & 0.0274 & 0.0206 & 0.0162 \\
\hline
\end{tabular}


with burnup decreases with the buildup of U-233 from the decay of Pa-233. During the first month or two of core operation, a great deal of U-233 is tied up in the Pa-233 which has a half life of about 27 days.

The actual core is not homogeneous but consists of several fuel regions and a fertile blanket, all separated by solid graphite. Also, after the first few core loadings, equilibrium concentrations of U-233 and $\mathrm{Pa}-233$ are present in the blanket. Since the blanket is only partially unloaded every time the core is unloaded, these equilibrium concentrations are present through most of the reactor's existence. Therefore, the hot $\left(1200^{\circ} \mathrm{F}\right)$ initial reactivity with equilibrium xenon present was recalculated at the beginning of a core life using a multiregion model and an equilibrium concentration of the various isotopes in the blanket. This calculational point is indicated on Figure 1.4-14 by the symbol B. Physically what is happening is that the clean fertile blanket is not as good a reflector as the reflector savings assumed in the equivalent bare model, thus the reactivity decreases. When fuel is built up in the blanket, the reactivity increases and the net result is a slight decrease in the overall reactivity as compared to the equivalent bare calculations.

From Figure 1.4-14, it is seen that the homogeneous model predicts a $K_{\text {eff }}$ of about 1.00 after 100 days of core life. The effect of non-uniform burnup is not expected to be very severe because the reactor is building up fuel faster in the region of high burnup than in other regions. The presence of fuel in the blanket will tend to offset the effects of nonuniform burnup.

\subsubsection{Neutron Spectrum in the Core}

The calculation of the neutron spectrum is based on the homogenized core composition. The relative fast neutron flux per unit lethargy was determined for each of the 54 groups as outlined in Section 1.2.2. The thermal neutron spectrum was determined as indicated in Section 1.2.5. The fast and thermal flux distribution were coupled by

$$
\frac{C \sum_{n=1}^{54} F_{n} \Delta_{n}}{\bar{\phi}_{t h}}=\left[\frac{D_{s} B^{2}+\Sigma_{a}^{T}}{\rho D_{f}}\right] \tau
$$




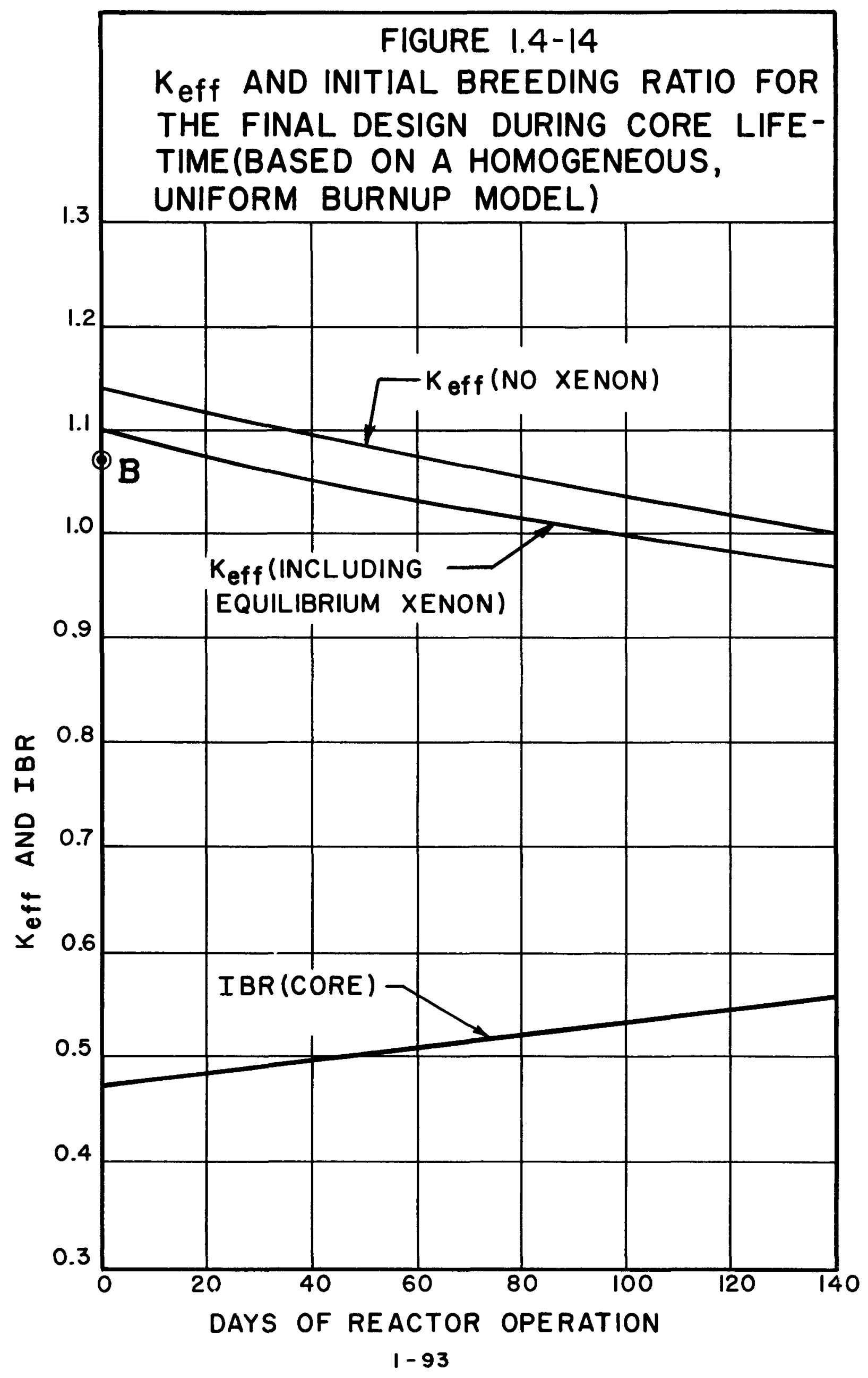


where the constant $C$ is the appropriate scaling factor to make the relative total fast flux consistent with the relative thermal flux.

The neutron flux spectrum is shown plotted in Figure 1.4-15 where the ordinate is relative flux per unit lethargy.

\subsubsection{Temperature Coefficient of Reactivity}

The temperature coefficient was determined by two multiregion, two-group calculations, one at $1200^{\circ} \mathrm{F}$ (assumed operating temperature) and the other $1400^{\circ} \mathrm{F}$. In each case, Doppler broadened cross-sections were used at the appropriate temperatures and equilibrium xenon was included. The temperature coefficient based on the gross reactivity change is:

$$
\frac{\Delta e}{\Delta T}=-2.70 \times 10^{-5} /{ }^{\circ} \mathrm{F}
$$


NEUTRON SPECTRUM FOR FINAL DESIGN AT $T=1200^{\circ} \mathrm{F}$ BASED ON HOMOGENEOUS MODEL

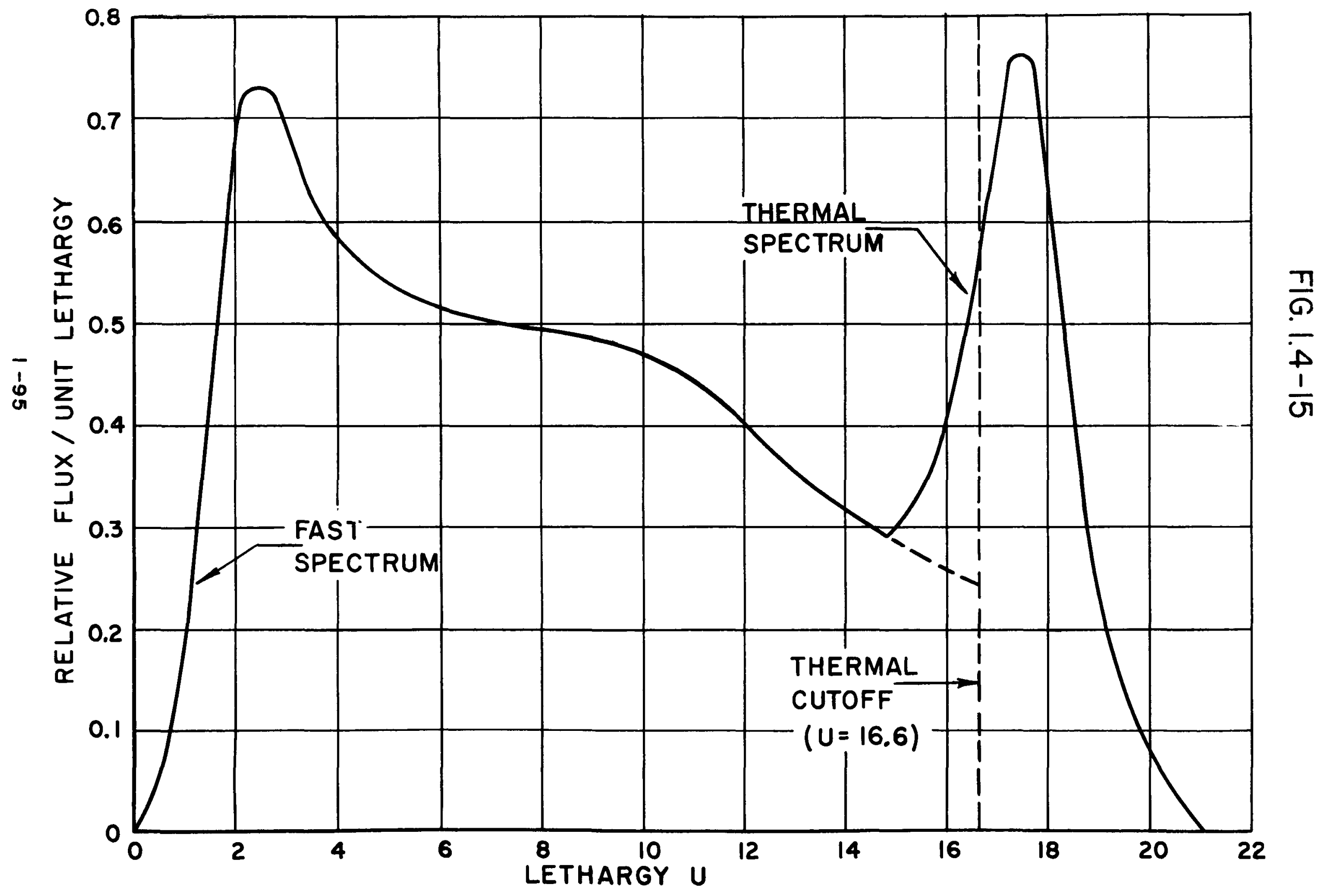


1.4.9 Summary of Reactor Characteristics

TABLE $1.4-3$

Réactor Characteristics for Final Design

Parameter

Material Content

Oxide Weight Percent

in Fuel Elements

$\mathrm{U}-233$ Loading $(\mathrm{Kg})$

Th-232 Loading $(\mathrm{Kg})$

$\mathrm{Th} / \mathrm{U}$ Atom Ratio

Void Fractions

Volume $\left(\mathrm{cm}^{3} \times 10^{-6}\right)$

Nuclear Properties

$\left(1200^{\circ} \mathrm{F} ., \mathrm{E}_{4}\right.$. Xe):

$\top$

$\mathrm{p}$

$\mathrm{D}_{1}$

$\mathrm{D}_{2}$

$\mathrm{K}_{\mathrm{f}}$

$\mathrm{K}_{\mathrm{th}}$

$\Sigma_{\mathrm{a} 2}$

$\mathrm{IBR}$

$$
\frac{\text { Fuel Region 1 }}{\mathrm{C}-\mathrm{UO}_{2}-\mathrm{ThO}_{2}}
$$

17.3

190.0

11

0.39

2.08

736.9

0.339

1.955

1. 320

0.827

1.918

0.00503

0.124

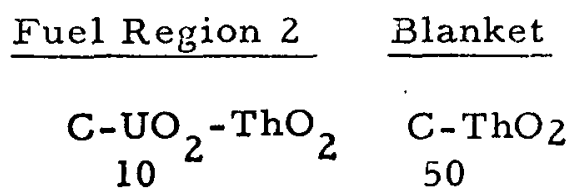

72.9

802.2

11

$0.32 *$

10.77

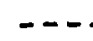

11,780

0.39

15.18

Overall Core Properties
Fuel Burnup Rate (Kg/MWYR)
0.485
Initial Capture to Fission Ratio
Fraction of Epithermal Fissions
0.257
Average Power Density (watts/cc)|
0.372
Percent Atom Burnup:
23.7
Core (After 100 Days)
0.021
Blanket (After 4 Years)
0.101
Control Rods:
Number
12
Reactivity Worth (Initial)
17.3
Material
Haynes -25

603.6

556.6

0.457

0.004

1.735

2. 047

1. 183

1. 303

0.869

1.858

-...

0.00423

0.00508

0.353

0.353

* The graphite web between the fuel pockets is humogenized with the fuel. 
TABLE $1.4-3$ (continued)

$$
\begin{aligned}
& \text { Temperature Coefficient } /{ }^{\circ} \mathrm{F} \\
& \text { Initial Multiplication Factor } \\
& \mathrm{K}_{\text {eff }}\left(1200^{\circ} \mathrm{F} ., \text { Ey. Xe }\right)^{*} \\
& \mathrm{~K}_{\text {eff }}\left(1200^{\circ} \mathrm{F} ., \mathrm{E}, \mathrm{Xe}\right)^{* * * *} \\
& \mathrm{~K}_{\mathrm{eff}}\left(77^{\circ} \mathrm{F} . \text {, No Xe }\right)
\end{aligned}
$$

$-2.7 \times 10^{-5}$

1. 100

1.070

1. 204

* Equivalent bare homogenized core model

*** Heterogeneous multiregion with equilibrium blanket composition 


\section{5 Shielding Analysis}

\subsubsection{Shutdown Shielding Requirements}

The plant shield design is based on the premise that the vapor con-. tainer will be inaccessible during power production. However, the operating floor above the reactor vessel must be accessible at shutdown periods for core refueling and equipment maintenance.

The major source to be shielded during shutdown is the fission product gammas in the spent core. Reference (19) describes the decay of fission products and the resultant gamma flux as a function of time after shutdown. The gamma flux curves after infinite reactor operation were used for the computation. This results in a slightly conservative estimate of the gamma source, but obviates the necessity for knowing the exact core lifetime.

The gamma flux in seven energy groups was included in the shield analysis. The model used was a uniform cylindrical source shielded on one end by a slab shield. The slab shield consisted of a steel lamination $2-3 / 4 "$ thick and a concrete lamination which was allowed to vary from two to ten feet in thickness. Gamma fluxes at 2, 6 and 10 hours after shutdown were used. The equation from $(20)$ for an end shielded cylinder is:

$$
\phi_{\gamma}=\frac{B S_{v}}{2 \mu_{s}}\left[E_{2}\left(b_{1}\right)-\frac{E_{2}\left(b_{1} \sec \theta_{1}\right.}{\sec \theta_{1}}\right]
$$

The results are shown in Figure 1.5-1 where the total dose at the surface of the concrete is expressed as a function of the concrete thickness above the core for the three time increments investigated. This curve indicates that four feet of concrete will provide an adequate shield above the core.

\subsubsection{Primary Shielding}

The primary shield must fulfill two purposes:

1. Reduce the neutron flux to a level which will not cause excessive induced activity in componcnts external to the reactor core. 
FIG. I.5-I

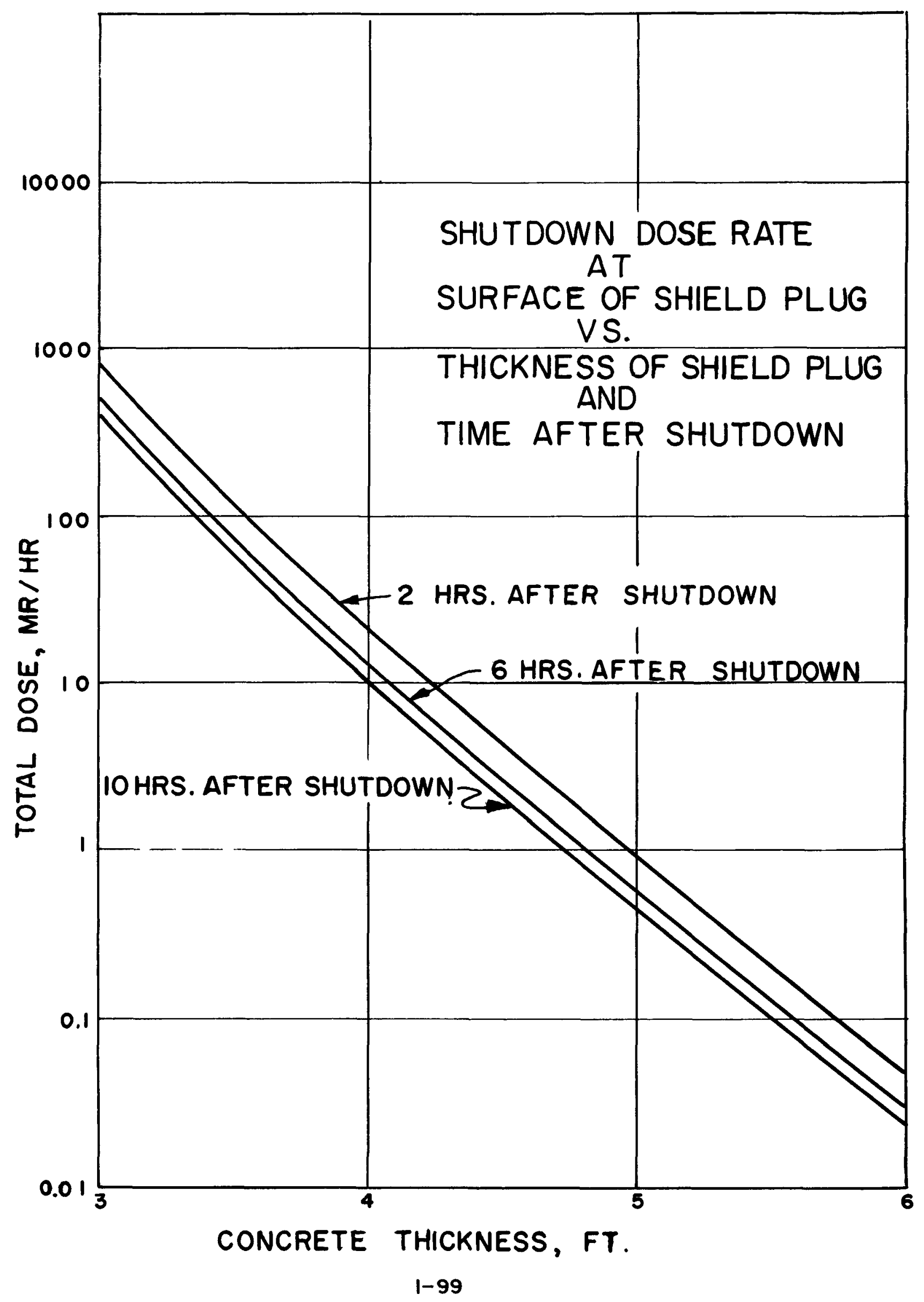


FIG. I.5-2

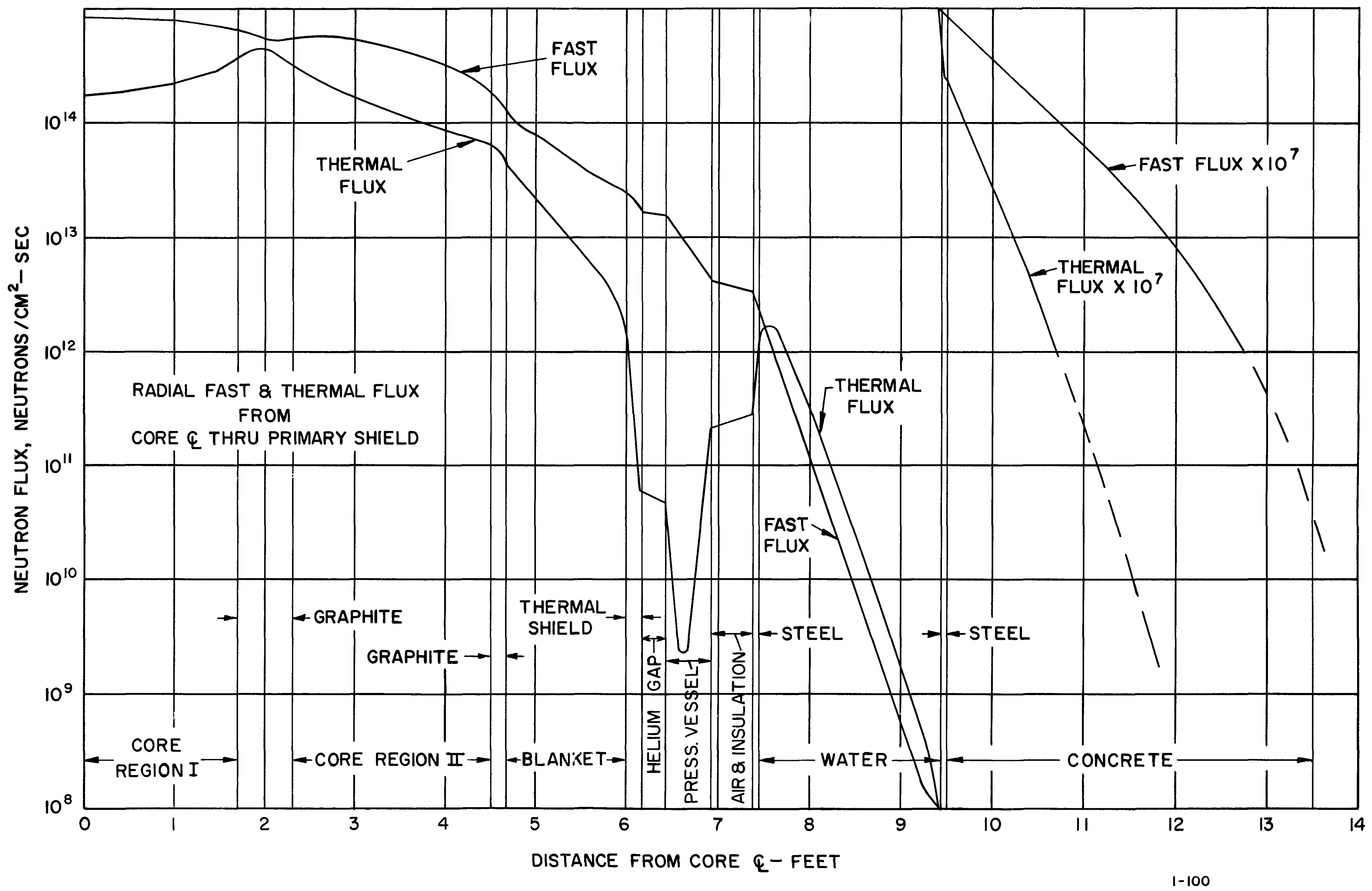


2. Attenuate the core gamma flux to a level at or below that of other components in the primary loop.

The radial neutron flux distribution through the primary shield was calculated using two groups of neutrons in a multiregion calculation. Thirteen regions were used. The thermal and fast neutron fluxes are plotted in Figure 1.5-2 from the core centerline through the thirteen regions, the last region consisting of four feet of concrete as the primary shield. The fast neutron flux penetrating the four feet is approximately $5 \times 10^{3}$ neutrons $/ \mathrm{cm}^{2}-\mathrm{sec}$. This level of flux should not cause any induced activity problem. The thermal flux at the surface of the concrete will be negligible.

The major gamma contributors at the surface of the primary shield were assumed to be full power core gammas, capture gammas in the water and capture gammas in the concrete shield. The total fission and capture core gamma spectrum was assumed to be $20 \mathrm{mev}$ per fission, with a typical energy of 1.5 mev per photon. This assumption is felt to be reasonable after reviewing the atom densities and capture cross-sections of materials in the core.

The equation used to calculate the core gamma contribution at the surface of the primary shield was:

$$
\phi_{\gamma}=\frac{B S_{v} R_{0}^{2}}{2(a+z)} F\left(\theta, b_{2}\right)
$$

The core gamma contribution with two feet of concrete was only $8.6 \mathrm{mr} / \mathrm{hr}$ and therefore, the calculation was not extended.

Capture gammas originating in the water tank were computed using an exponential approximation of the neutron flux in the water. The volume source thus becomes:

$$
S_{v}=\phi_{n_{0}} \sum_{a} e^{-a t} C^{\prime \prime}
$$

A best fit approximation gives an equivalent volume source of:

$$
S_{v}=8.708 \times 10^{10} e^{-0.114 t}
$$


This source term was used in the infinite slab source equation which is:

$$
\phi_{\gamma}=\frac{B S_{r} e^{k b_{3} / \mu_{s}}}{2 \mu_{s}}\left[F_{1}\left(b_{3}, \frac{-k}{\mu_{s}}\right)-F_{1}\left(b_{1}, \frac{-k}{\mu_{s}}\right)\right]
$$

With 2 feet of concrete shield, the water contribution was only $2 \times 10^{-3}$ $\mathrm{mr} / \mathrm{hr}$ and therefore, this calculation was also not extended.

The third source of full power gammas investigated was capture gammas in the concrete. Again, the neutron flux creating the source was approximated by an exponential and the equivalent gamma source was used in the equation:

$$
\phi_{\gamma}=\frac{B \delta_{e^{k h}}}{2 \mu_{s}} F_{1}\left(\mu_{s} h_{,} \frac{-k}{\mu_{s}}\right)
$$

which is the equation for an infinite slab source for the special case of the dose point at the surface of the slab.

Calculations were made with 2 and 4 feet of concrete. The gamma contribution from the concrete with $2 \mathrm{ft}$. of concrete was $280 \mathrm{mr} / \mathrm{hr}$. With $4 \mathrm{ft}$. of concrete, the contribution was $8 \mathrm{mr} / \mathrm{hr}$.

The calculations indicate that the primary shield thickness should be primarily based on attenuating the fast neutron flux. Four feet of concrete appears adequate for this purpose and is used for the preliminary shield design. One source of gammas which was not investigated for the preliminary design, but which may be important, is the capture gammas in the structural steel outside the core, namely the thermal shield, pressure vessel and the steel which forms the water tank. It is expected that the four feet of concrete will adequately shield these capture gammas.

\subsubsection{Vapor Container Shielding}

The vapor container or secondary shielding design was based on a maximum system rupture. It was assumed that the primary system ruptured and that all fission products volatile at or below $2500^{\circ} \mathrm{F}$ were released to the vapor container. It was further assumed that the fission 
gases were uniformly distributed throughout the vapor container. This gave a uniform cylindrical volume source to shield for safe evacuation of the plant site.

Since the vapor container height had not yet been established, shielding calculations were made with height variable. Equation (1.5-2) was used to determine the dose at a point from a shielded cylindrical source.

Since the source consists of gases, the self-shielding of the source is negligible and the self-shielding distancc becomes zero. The volume isource $S_{V}$ is the total gamma emiision $\left(S_{0}\right)$ divided by the vapor container volume. Thus, equation $(1.5-2)$ can be rewritten as:

$$
\phi_{\gamma}=\frac{B S_{0}}{2_{a} \pi H} F(\theta, b)
$$

Equation (1.5-6) was solved for $H=30$ and $60 \mathrm{ft}$. The results are plotted in Figure 1.5-3 as dose rate in $R / h r$ as a function of distance from the vapor container for three concrete thicknesses, 1,2 , and 3 feet and for the two vapor container heights considered. Figure 1.5-3 shows that the dose rate is only slightly dependent on height as expected.

The plant layout indicates that the control room will be located approximately $75 \mathrm{ft}$. from the vapor container. A cross plot of Figure 1. 5-3 was made for this distance to give the dose rate at $75 \mathrm{ft}$. as a function of concrete thickness. This is illustrated in Figure 1.5-4. The maximum dose rate is $30 \mathrm{R} / \mathrm{hr}$. for a concrete thickness of $2-1 / 2$ ft. With no allowance for fission product decay, this would permit an evacuation time of 50 minutes without exceeding an emergency dose tolerance of $25 \mathrm{R} / \mathrm{hr}$. Also, the dose raterapidly decreases with dis tance, thus as personnel move out of the area, there is a further reduction in exposure. When fission product decay and added distance are accounted for, the total emergency exposure should be much less than $25 \mathrm{R}$. Therefore, $2-1 / 2$ feet was used as the reference secondary shield thickness. 
FIG. I.5-3

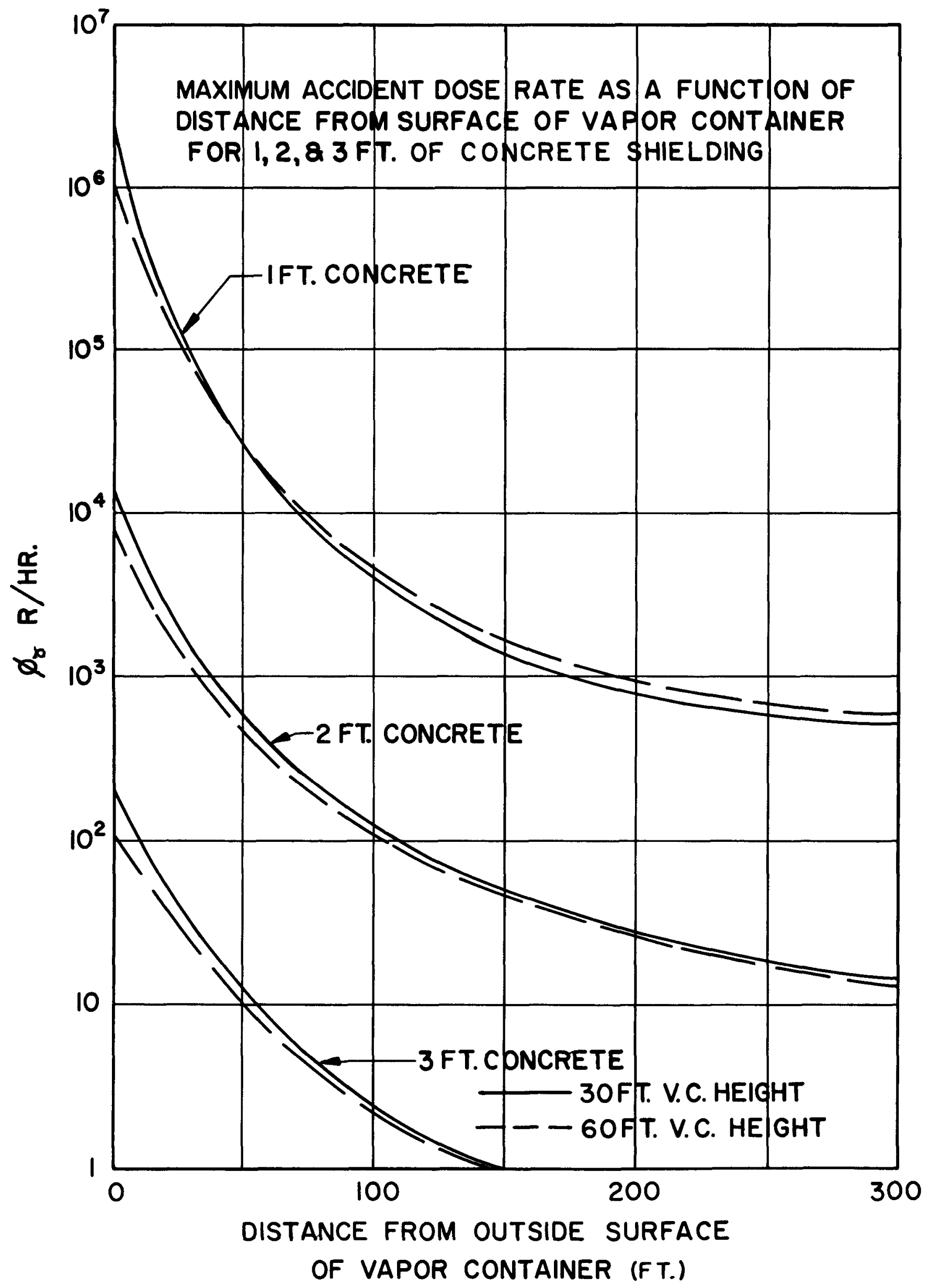




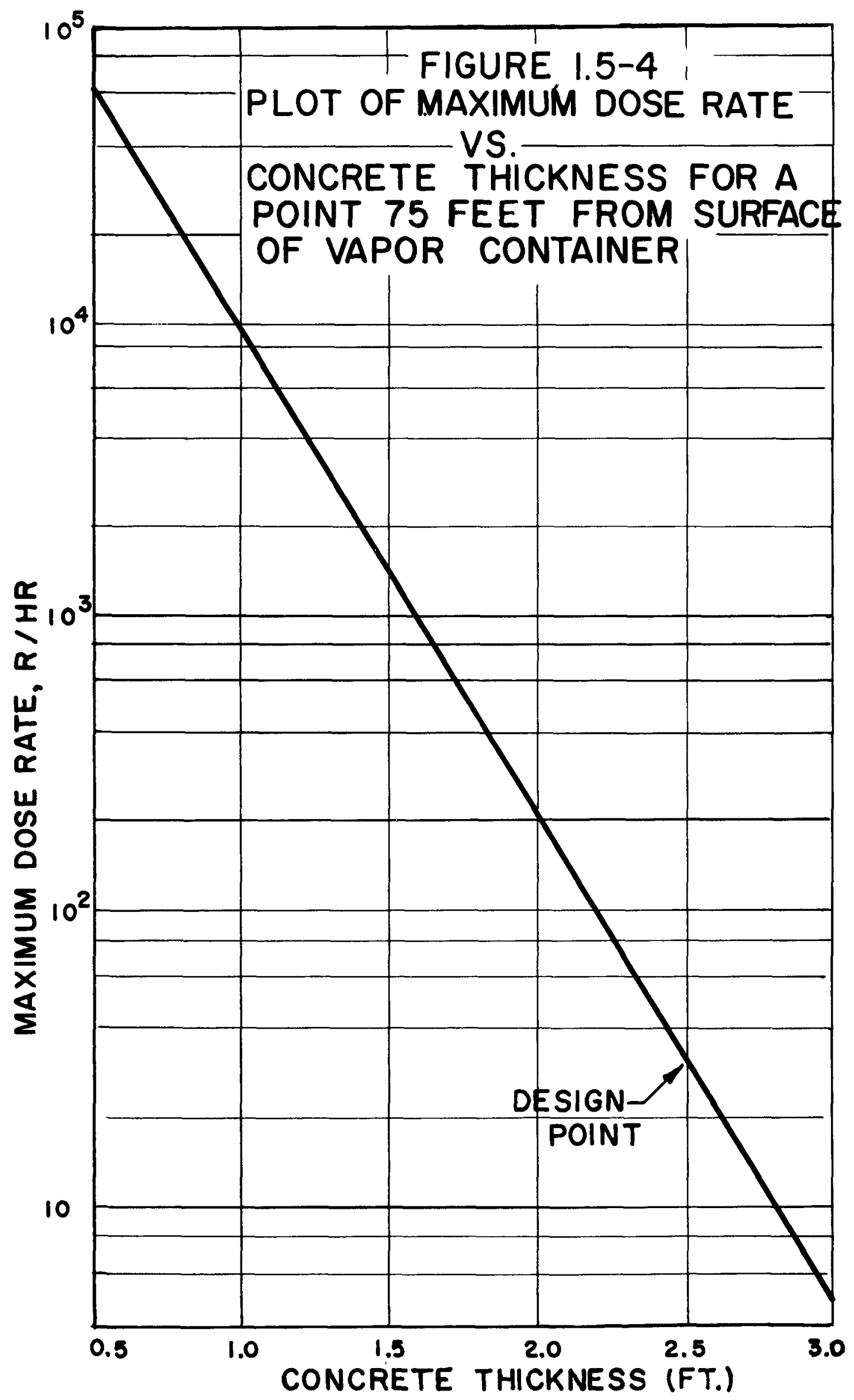

1-105 


\begin{tabular}{|c|c|c|}
\hline Symbol & Definition & Units \\
\hline $\mathrm{B}^{2}$ & Geometric buckling & $\mathrm{cm}^{-2}$ \\
\hline $\bar{\mu}$ & Average cosine of the scattering angle & \\
\hline $\bar{\xi}$ & $\begin{array}{l}\text { Average change of the logarithm of } \\
\text { lethargy in a collision }\end{array}$ & \\
\hline $\mathrm{J}_{\mathrm{n}}$ & Net current per unit lethargy & $\mathrm{n} / \mathrm{cm}^{2}-\sec$ \\
\hline $\mathrm{T}_{\mathrm{n}}$ & Neutrons produced per unit flux & \\
\hline$B$ & Buildup factor & \\
\hline$\mu$ & Attentuation coefficient & $\mathrm{cm}^{-1}$ \\
\hline$\theta$ & Half angle subtended by source & degrees \\
\hline $\mathrm{S}_{\mathrm{V}}$ & Volumetric source strength & $\mathrm{Mev} / \mathrm{cm}^{3}-\mathrm{sec}$ \\
\hline $\mathrm{t}$ & Thickness of shield material & $\mathrm{cm}$ \\
\hline $\mathrm{b}_{1}$ & $\sum_{i}^{n} \mu_{i} t_{i}$ & \\
\hline $\mathrm{h}$ & Slab source thickness & $\mathrm{cm}$ \\
\hline b3 & $b_{1}+\mu_{s} h$ & \\
\hline $\mathbf{z}$ & Self shielding distance & $\mathrm{cm}$ \\
\hline $\mathrm{a}$ & Distance from source to dose point & $\mathrm{cm}$ \\
\hline $\mathrm{b}_{2}$ & $\mathrm{~b}_{1}+\mu_{\mathbf{s}} \mathrm{z}$ & \\
\hline $\mathrm{H}$ & Height & $\mathrm{cm}$ \\
\hline$\Sigma_{\mathbf{a}}$ & \multicolumn{2}{|c|}{ Macroscopic neutron absorption cross-section $\mathrm{cm}^{-1}$} \\
\hline$\phi_{n_{0}}$ & Neutron flux at inner surface of source & $\mathrm{n} / \mathrm{cm}^{2}-\mathrm{sec}$ \\
\hline $\mathrm{C}^{\prime \prime}$ & Number of gammas per neutron capture & $\gamma / \mathrm{n}$ \\
\hline $\mathrm{e}^{\mathrm{kt}}$ & $\begin{array}{l}\text { Exponential fit of the neutron flux through } \\
\text { the source }\end{array}$ & \\
\hline$F_{n}(t, a)$ & Functions described in Reference (20) & \\
\hline$E_{n}(0)$ & Functions described in Reference $(\underline{20})$ & \\
\hline$F(\theta, b)$ & Functions decsribed in Reference $(\overline{20})$ & 2 \\
\hline$\phi \gamma$ & Gamma tlux & \\
\hline
\end{tabular}


REFERENCES FOR SECTION 1.0

(1). Hellens, R. L., R. W. Long, and B.H. Mount, "Multigroup Fourier Transform Calculation Description of MUFT III Code", WAPD-TM.44 (July, 1956)

(2) Murray, Raymond L., "Group Resonance Integrals and Crosssections for Fertile Materials", APAE Memo 116, Alco Products Inc., Nov. 1957.

(3) Hughes, Donald J. and John A. Harvey, Neutron Cross-sections, BNL-325, July 1,1955

(4) Hughes, Donald J. and Robert Schwartz, Neutron Cross-sections Supplement, BNL Supplement, No. 1, January 1957.

(5) Hughes, Donald J., Neutron Cross-sections, Pergamon Press Inc., New York (1957)

(6) Harvey, J.A., et al, "Spacings and Widths of Nuclear Energy Levels," Physics Review 99, 11 (1955)

(7) Blatt, J. and V. Weisskopf, Theoretical Nuclear Physics, John Wiley and Sons, Inc. New York, 1952

(8) Oleska, Sophie, "The Variation of a of U-235 with Energy in the Intermediate Energy Range," Journal of Nuclear Energy, Vol. 5, No. 1, p. 16 (1957)

(9) Murray, Raymond L., "Flux and Doppler Effect Self-shielding Factors for Th-232 and U-233 in Homogeneous Graphite-Moderated Reactors", APAE Memo 122, Alco Products Inc. Feb. 1958

(10) Glasstone, S. and M.C. Edlund, The Elements of Nuclear Reactor Theory, New York, D. Van Nostrand Company (1952)

(11) Rose, M.E., et al, "A Table of the Integral $\psi$ (x., t)," WAPD-SR-506, Volume I and II (October, 1954)

(12) Dresner, L., "The Effective Resonance Integrals of U-238 and Th-232," Nuclear Science \& Engineering, Vol. 1, No. 1, March 1956

(13) Oby, P.V., "Scram I: Control Rod Worth Calculation on the IBM-650" APAE Memo 89, Alco Products, Inc. April 1957

(14) Murray, Raymond L. and John W. Niestlie, "Reactor Control-Rod Theories", Nucleonics, February 1955, Vol. 13, No.2, pages 18-22

(15) Stark, R.H. and G.M. White, "VALPROD - A Revision of the PROD II 650 Multigroup Program," File Number 8.2.013, G. E. Atomic Power Equipment Department, Vallecitos Atomic Laboratory, Jan. 1957

(16) Fairbanks, F.B. "Two-Group Multiregion Axial Window Shade Calculation on the IBM-650", APAE Memo 88, Alco Products Inc. March 1957 
(17) "Program Progress Report", January and February, 1956, KAPL-1501, Confidential, March, 1956.

(18) Sampson, J.B. Et al., "Poisoning in Thermal Reactors Due to Stable Fission Products, "KAPL-1226 (1954)

(19) Clark, F.H. - "Decay of Fission Product Gammas," NDA-27-39, February 15, 1955

(20) Rockwell III, Theodore, Reactor Shielding Design Manual, McGrawHill Book Company, Inc., (1956)

(21) Cohen, E. Richard, "The Neutron Velocity Spectrum in a Heavy Moderator," NAA-SR-1940, October 15, 1957.

(22) Roberts, J.T., "Cross-Sections for OCUSOL-A Program", ORNL-CF-57-6-5(1957) 


\section{Section 2.0}

Thermal \& Hydraulic Analysis

2.1 - Characteristics of Fixed Pebble Beds............... 2-1

2.1.1 - Physical Characteristics of Spherical Particles.... 2-1

2. 1.2 - Physical Characteristics of Randomly Packed Beds. 2-3

2.1.3 - Fluid Dynamics of Randomly Packed Beds......... 2-9

2.1.4 - Thermal Characteristics of Randomly Packed Beds. 2-13

2. 1.5 - Application of Pebble Beds to Nuclear Reactors . . 2-15

2.2 - Thermal and Hydraulic Analysis of Pebble Beds........ 2-18

$2.3-$ Selection of Fuel Element Size ................. 2-27

2.4 - Thermal and Hydraulic Design of Final Core...........2-31

Nomenclature. .............................2-46

References............................. $2-47$ 


\subsection{Thermal \& Hydraulic Analysis}

This section presents some pertinent characteristics of spherical particles and beds of spherical particles. The thermal and hydraulic survey which was carried out in studying the 350 tMW cores is presented and finally, a detail discussion and analysis of the final core with respect to its thermal and hydraulic performance.

\section{1 Characteristics of Fixed Pebble Beds}

Characteristics of beds of particles have been under investigation for the past fifty years. In general this work has covered many types of fluids flowing through materials ranging from sand to coarse aggregate. There has been occasional work on spherical particles which is of direct interest in the study of randomly packed pebble beds as proposed for the PBR 125 eMW plant. Reference (1) to (14).

One of the greatest shortcomings of the work done to date is the fact that the bulk of the data concerns the characteristics of beds of small particles (i.e. less than one tenth of an inch in diameter) at low Reynolds numbers (i.e. less than 10,000). Both of these ranges are below the regions in which our interest lies.

An attempt has been made to report the best interpretation of the data available, recognizing that it is taken from many sources in which the objective of the experiment and the method of reporting and interpreting are not consistent. From these data we have selected parameters for design.

\section{1. I Physical Characteristics of Spherical Particles}

The following fundamental formulae relate various properties of spheres to the basic sphere and/or bed dimensions. For convenience each formula is coded with a number (the primed numbers refer to a special case of the general formula bearing the same number).

Surface area of sphere

$$
\mathrm{s}=\pi \mathrm{D}_{\mathrm{p}}{ }^{2}
$$


Volume of sphere

$$
v=\pi D_{p}^{3} / 6
$$

Average voidage in a random-packed bed of large $d / D_{p}$

$$
\varepsilon=39 \%
$$

Packing fraction in a random-packed bed $(\varepsilon=.39)$

$$
1-\varepsilon=61 \%
$$

Number of spheres per unit bed volume

$$
\begin{aligned}
& z=6(1-\varepsilon) / \pi D_{p}^{3} \\
& z=6 \times \begin{array}{c}
.61 \times 1728 / \pi D_{p}^{3}=2013 / D_{p}^{3} \text { per } F t^{3} \\
\text { where } D_{p} \text { is in inches }
\end{array}
\end{aligned}
$$

Sphere surface area per unit bed volume

$$
\begin{aligned}
& S=6(1-\varepsilon) / D_{p} \\
& \mathrm{~S}=6 \times .61 \times 12 / \mathrm{D}_{\mathrm{p}}=43.92 / \mathrm{D}_{\mathrm{p}} \quad \mathrm{Ft}^{2} / \mathrm{Ft}^{3}
\end{aligned}
$$

Sphere surface area per unit void volume, i.e. per unit volume of bed interstices

$$
\begin{aligned}
S^{\prime} & =6(1-\varepsilon) / \varepsilon D_{p} \\
s^{\prime} & =6 \times \begin{array}{l}
.61 \times 12 / .39 D_{p}=112.6 / D_{p} \\
\text { where } D_{p} \text { is in inches }
\end{array} \mathrm{Ft}^{2} / \mathrm{Ft}^{3}
\end{aligned}
$$

Equivalent diameter of interstitial pores, i.e. flow passage

$$
\begin{aligned}
\phi= & \frac{4 D_{p} \varepsilon}{6(1-\varepsilon)} \\
\phi= & 4 \times \cdot \begin{array}{l}
39 D_{p} / 6 \times .61=D_{p} / 2.35 \\
\text { where } D_{p} \text { is in inches }
\end{array}
\end{aligned}
$$

The above characteristics are tabulated in Table 2.1-1 below for a range of sphere diameters of interest. 
TABLE 2.1.1

Characteristics of Beds of Spheres

$\begin{array}{lcccc}\mathrm{D}_{\mathrm{p}} \text { (inches) } & \phi \text { (inches) } & \mathrm{z} & \mathrm{S}\left(\mathrm{Ft}^{2} / \mathrm{Ft}^{3}\right) & \mathrm{S}^{\prime}\left(\mathrm{Ft}^{2} / \mathrm{Ft}^{3} \text { voids }\right) \\ 1 / 2 & .213 & 16100 & 87.8 & 225.0 \\ 5 / 8 & .266 & 8250 & 70.3 & 180.3 \\ 3 / 4 & .319 & 4780 & 58.6 & 150.2 \\ 7 / 8 & .372 & 3005 & 50.2 & 128.7 \\ 1 & .425 & 2013 & 43.9 & 112.6 \\ 1-1 / 8 & .479 & 1415 & 39.0 & 100.1 \\ 1-1 / 4 & .532 & 1030 & 35.3 & 90.1 \\ 1-3 / 8 & .585 & 775 & 31.9 & 81.9 \\ 1-1 / 2 & .638 & 597 & 29.3 & 75.1\end{array}$

2.1.2 Physical Characteristics of Randomly Packed Beds

The principal physical characteristic of randomly packed beds is the voidage $\varepsilon$. $l-\varepsilon$ is the packing fraction or the percentage of the bed filled with solids. $\varepsilon$ is independent of the size of particle $D_{p}$ but is dependent upon the ratio of the diameter of the container $d$ to the size of the particle. As would be expected $\varepsilon$ varies radially through the bed due to wall effect and the magnitude of this variation is dependent upon the ratio $d / D_{p}$.

The absolute value of $\varepsilon$ selected for design influences the absolute magnitude of the pressure drop and the film coefficient. It has but a second order effect on the ratio of pumping power to heat transferred.

Voidage of a finite bed of a size where the diameter of the container is greater than about ten times the size of the particle is most often reported as $39 \%$ and we have used this value for design. Denton (1), who has done the most work in this field on configurations which more closely approach our requirements, reports that in repeated packings the most 
probable variation in voidage is $\pm .05 \%$ around a mean of $39.1 \%$. The point has been raised regarding the effect of occasional broken balls on pressure drop, or voidage, and we are inclined to believe it will be between these limits.

Voidage of an infinite bed (i.e. characteristic voidage) is reported between 30 and $38 \%$. These values are invariably deduced from a value of the infinite bed and an estimate of wall voidage. Figure 2.1-1 presents data of Carman (2) indicating that the voidage of an infinite bed, or the characteristic voidage, is of the order of $38 \%$. We have accepted this value as being representative.

The difference between the characteristic voidage of an infinite bed $(38 \%)$ and the voidage of a finite bed $(39 \%)$ is due to wall effect. Simple geometrical considerations indicate that near the wall of the containing vessel the flow channels are larger in area therefore causing a greater voidage at the wall than near the center of the bed. This voidage is maximum at a $d / D_{p}$ ratio slightly less than 2 and minimum for a $\mathrm{d} / \mathrm{D}_{\mathrm{p}}$ ratio of infinity. This effect is shown in Figure 2. 1-1.

The magnitude of wall voidage is the least known and least understood of pebble bed characteristics and from our standpoint is most important in reactor design. Wall voidage is probably a maximum about one sphere diameter in from the wall but characteristic voidage is not reached for two or possibly three sphere diameters.

Schwartz (3) has done work in this area, reported in Figure 2. 1-2, indicating that the wall effect extends for about two sphere diameters in from the wall. Although this is based on tests covering a $d / D_{p}$ ratio of only 8 to 16 , it clearly shows that wall voidage is a function of $d / D_{p}$ ratio. It is interesting to note that over the range of these experiments wall voidage is constant at $40 \%$ at one and one quarter ball diameters from the wall. However, the trend of the curve indicates a characteristic voidage from 30 to $33 \%$.

Consideration has been given to mixing two sizes of spheres in either the core (or blanket) where the smaller sphere contains the fissile and fertile (or fertile) material. When two sizes of spheres are mixed, and especially when the size ratio exceeds four, the mixture shows a lower porosity than either of its constituents. This is shown in Figure 2. 1-3. The surface area in a mixed bed in $\mathrm{Ft}^{2}$ surface per $\mathrm{Ft}^{3}$ of bed volume is equal to: 
CARMAN'S RELATION BETWEEN POROSITY OF BED F SIZE OF CONTAINER

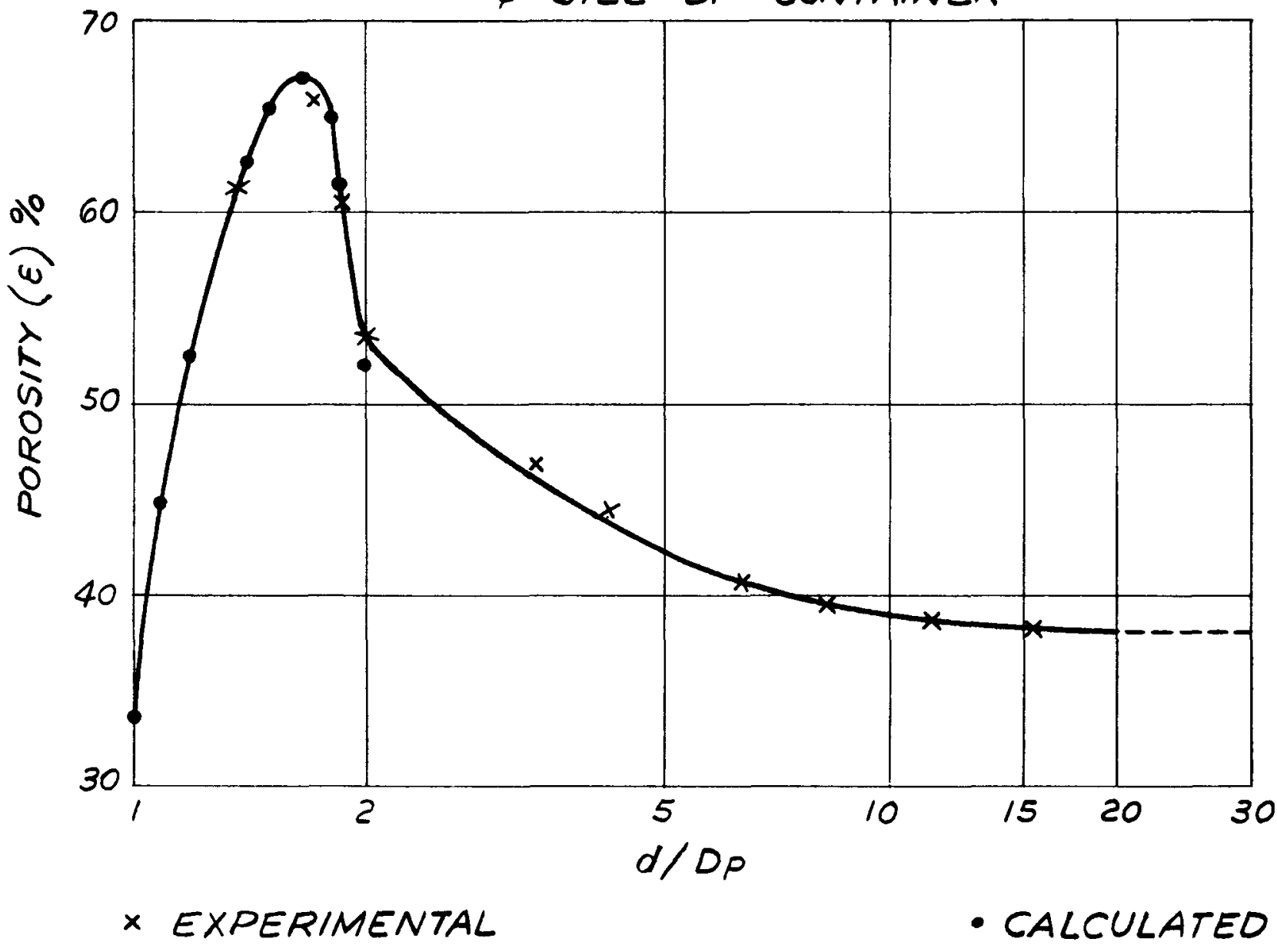


FIG. 2.1-2
FIG. 2.1-3

CAULSON'S RESULTS FOR MIXTURES

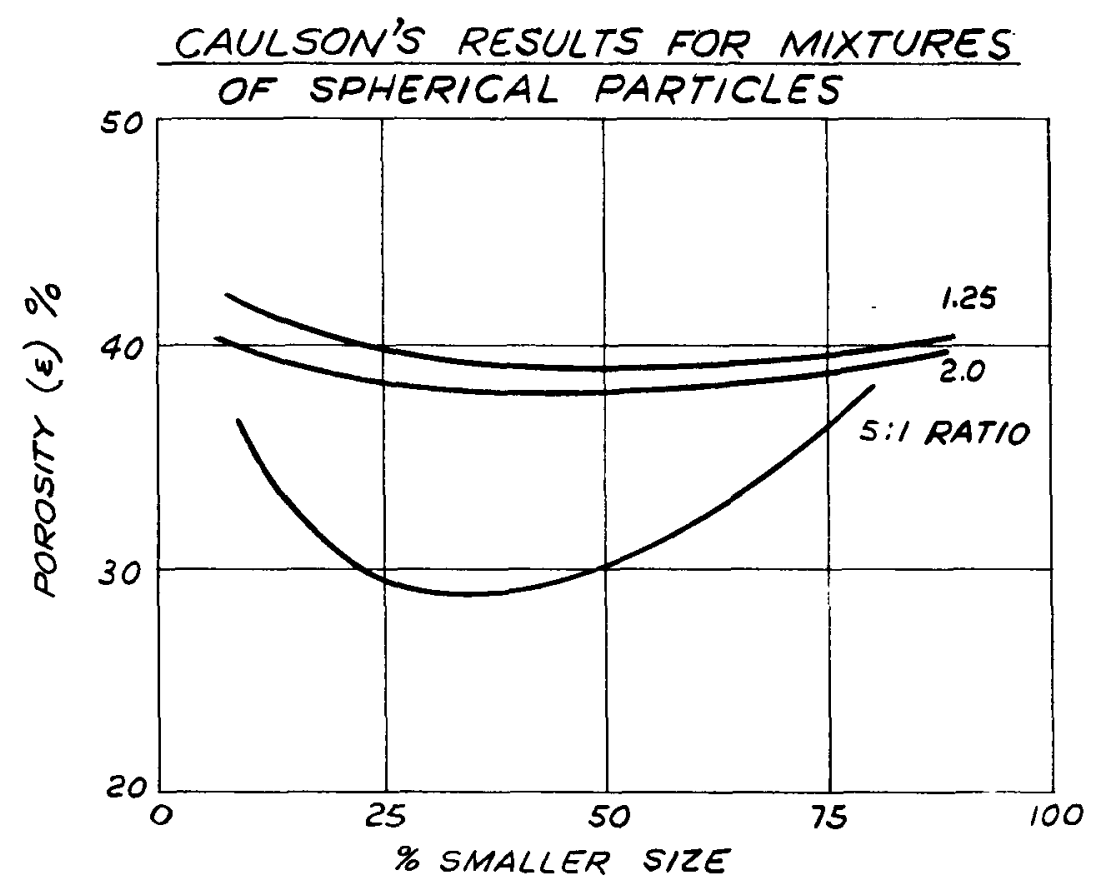

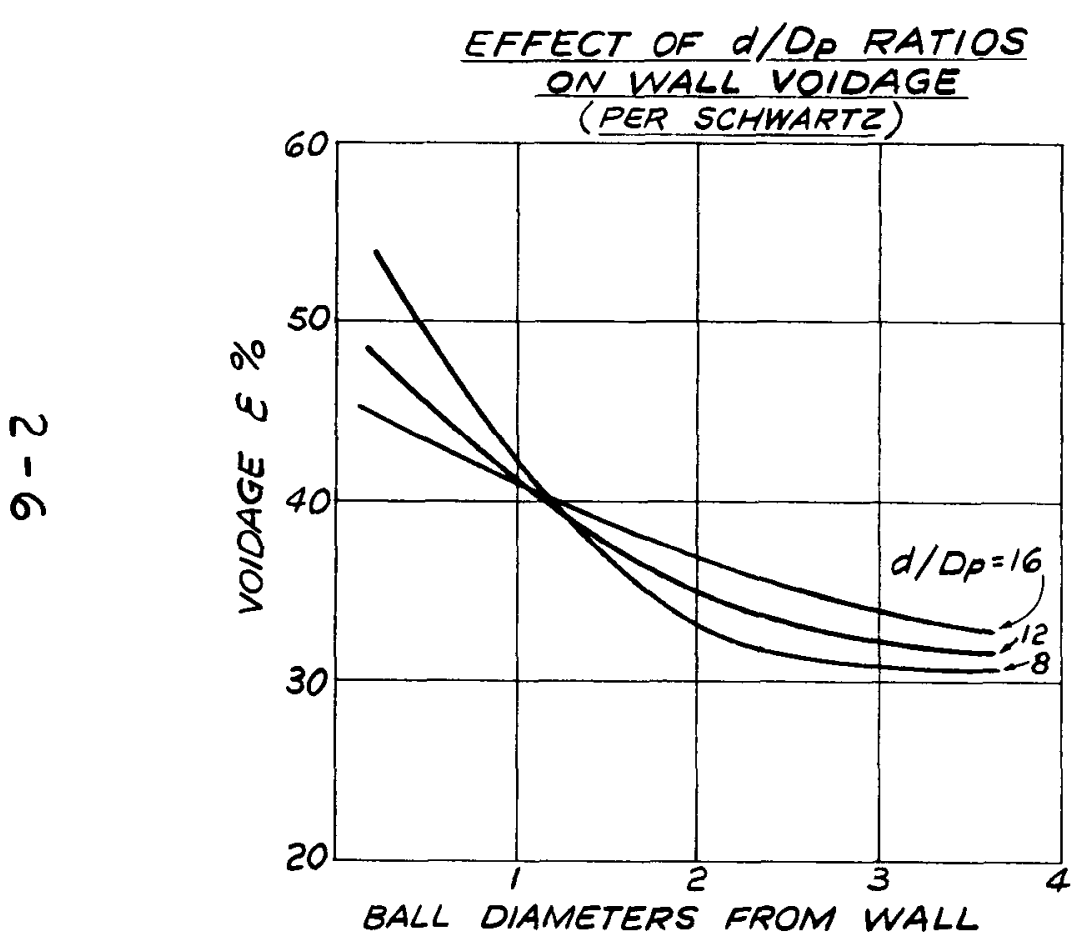

n

6) 


$$
S=6(1-\varepsilon) \Sigma \frac{V^{1}}{D_{p}}
$$

where $\mathrm{V}^{1}$ is the fractional volume of the particles of size $\mathrm{D}_{\mathrm{p}}{ }^{1}$. This approach has the objective of separating the fissile material from the bulk of the moderator. However, its use must await experimental work on the uniformity of distribution of fissile material throughout the core.

Mechanical characteristics of a packed bed which are of interest relate to the flow characteristics of spheres during loading and unloading of the reactor. Flow of particulate matter through pipes and ducts is well understood and practiced in industry. Spheres are usually handled in an open trough, not applicable to a reactor design. This has prompted an investigation of the flow of spheres in pipes by others.

The California Research Corporation (4) has reported some work on bridging of spheres while flowing through a tube. The surface finish and hardness of both the spheres and tube had a direct bearing on results. They concluded that bridging was impossible at $d / D_{p}$ ratios between 1 and 1. 75 , unlikely at $d / D_{p}$ ratios of 5 , and again highly improbable at $d / D_{p}$ ratios greater than 6. Therefore, we have used $d / D_{p}$ ratios of 1 to 1.75 and greater than 6 in our design.

Denton ( 1 ), in his study of the pebble bed, found that if a hemispherical bottom was put on a container $\left(d / D_{p} \sim 40\right)$ uninterrupted flow was obtainable through an outlet pipe of $3 \mathrm{D}_{\mathrm{p}}$ in diameter.

The behavior of particulate matter in handling is well known in the conveying and handling industries. The angle of repose is a function of the shape, size, hardness and density as well as the hardness of the surface on which it is poured. Ideally, spherical particles have a zero angle of repose when released through a gate, i.e. they behave like liquids. However, unlike liquids, they cannot rise to seek their own level in interconnected regions, as shown in Figure 2.1-4. A further characteristic of the flow of spheres is that since when they are restrained they cannot rise to seek their own level, adjacent compartments separated by a gate can be drained in sequence as also shown in Figure 2.1-4. The number of interconnected compartments can be infinite and they will drain in sequence from the compartment in which the drain valve is located.

Valves for controlling the flow of spheres should not crush the spheres on closing due to the danger of blockage. The principles cited above:can be used to design a valve capable of controlling the flow of spherical particles in the same manner that a gate valve controls the flow of a liquid. 
FIG, $2,1-4$

\section{BALL FLOW CHARACTERISTICS}

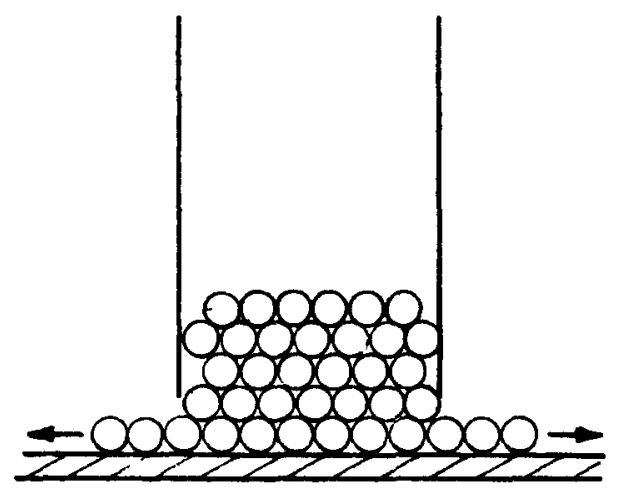

UNRESTRAINEO

SPHERICAL PARTICLES

HAVE A ZERO ANGLE

OF REPOSE, i.e.

THEY BEHAVE LIKE LIQUUIOS

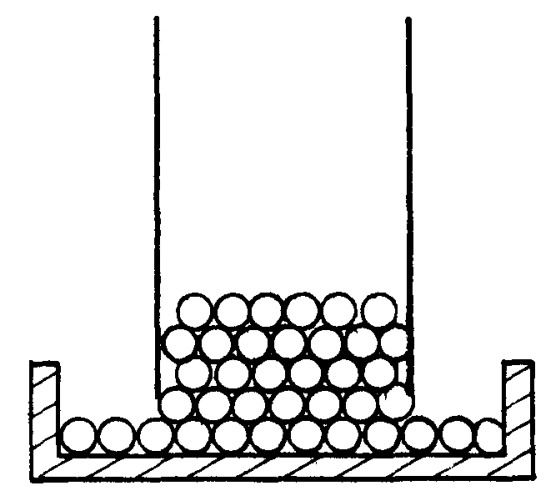

RESTRAINED SPHERICAL PARTICLES CANNOT RISE TO SEEK THEIR OWN LEVEL
COMPARTMENT IN WHICH VALVE IS LOCATED WILL EMPTY FIRST

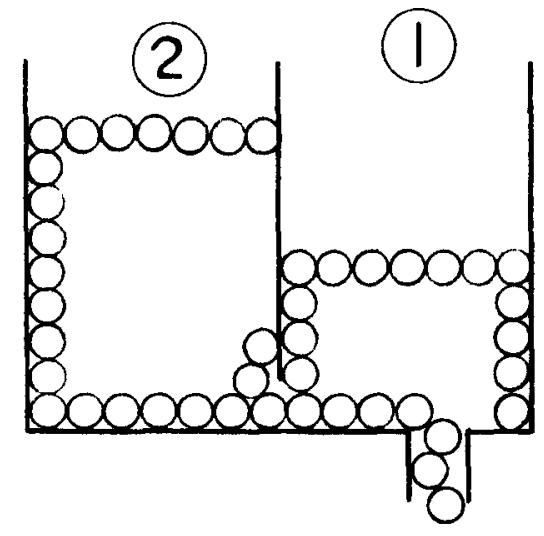

SINCE RESTRAINED, THEY CANNOT RISE TO SEEK THEIR OWN LEVEL
NO BALLS WILL FLOW FROM COMPARTMENT (2) UNTIL LEVEL OF 1 IS BELOW GATE

(2)

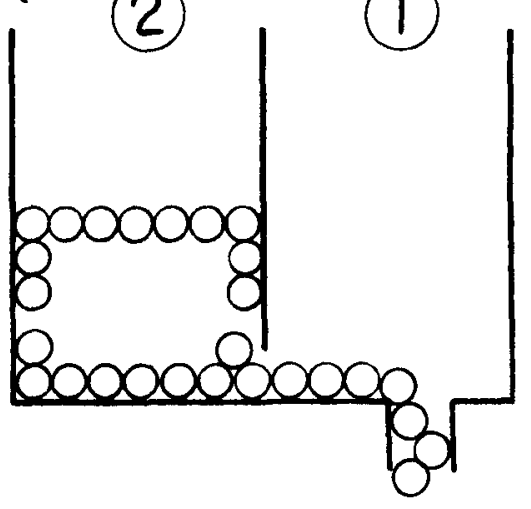

INTERCONNECTED COMPARTMENTS CAN BE EMPTIED BY CASCADING 


\section{1. 3 Fluid Dynamic Characteristics of Randornly Packed Beds}

Pressure drop through beds of randomly packed spheres is usually correlated by plotting a modified friction factor $f^{\prime}$ vs. a modified Reynolds number $\mathrm{Re}^{\prime}$ defined as follows:

$$
\begin{aligned}
& \mathrm{Re}^{\prime}=\frac{D_{p} G_{s}}{\mu(1-\varepsilon)} \\
& f^{\prime}=\frac{d p}{d L} \frac{\rho g D_{p}}{2 G_{s}} \frac{\varepsilon^{3}}{1-\varepsilon}
\end{aligned}
$$

Figure 2. 1-5 presents a plot of friction factors $f^{\prime} v s . \mathrm{Re}^{\prime}$ for testś on pebble beds as conducted by four investigators: Kayes \& London (5), Denton, Robinson \& Tibbs (6), Glasser \& Thodos (7), and Brownell, Dombrowski \& Dickey (8). These data show a spread of $\pm 25 \%$ around a mean line. Using $125 \%$ of the mean line for design, results in the following expression for $f^{\prime}$ :

$$
f^{\prime}=7.5 / \mathrm{Re}^{1.27}
$$

Since our survey work will be based on beds with a uniform voidage of $39 \%$, this value is substituted in the expression for $f^{\prime}$ and $R e^{\prime}$ to give:

$$
f=67.5 / \mathrm{Re}^{.27}
$$

where

$$
f=\frac{d p}{d L} \frac{g \rho D_{p}}{2 G_{s}}
$$

and

$$
\operatorname{Re}=D_{p} G_{s} / \mu
$$

Equation (2.1-13) is the relationship used for design and it is platted in Figure 2.1-6.

In selecting a value of the friction factor for design, the upper limit of the mean tolerance of $\pm 25 \%$ has been selected because of uncertainties at higher Reynolds numbers. We have found only two investigators who have established friction factors at Reynolds numbers in excess of 10,000, Denton (6) and Rose (9). While Denton's data was obtained at an acceptable $d / D_{p}$ ratio, Rose's data was at a much lower value than our range of 
FIG. 2.1 - 5

FRICTION FACTOR CORRELATION

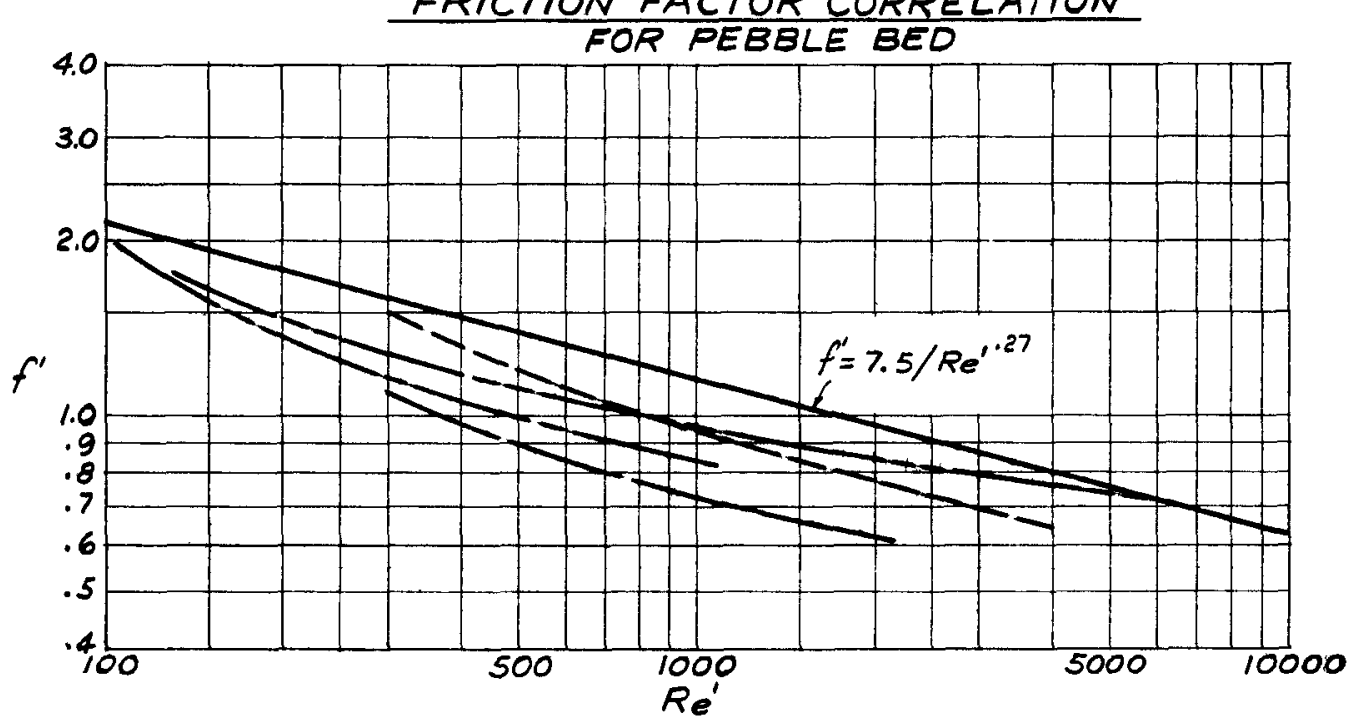

$f^{\prime}=\frac{d p}{d L} \cdot \frac{g \rho D p}{2 G_{s}^{2}} \cdot \frac{\varepsilon^{3}}{1-\varepsilon}$

LONDON KAYE

$R_{e}^{\prime}=\frac{D_{p} G_{s}}{\mu(1-\varepsilon)}$

DENTON ROBINSON TIBBS

GLASSER \& THODOS

BROWNELL DOMBROWSKI \& DICKEY

FIG. $2.1-6$

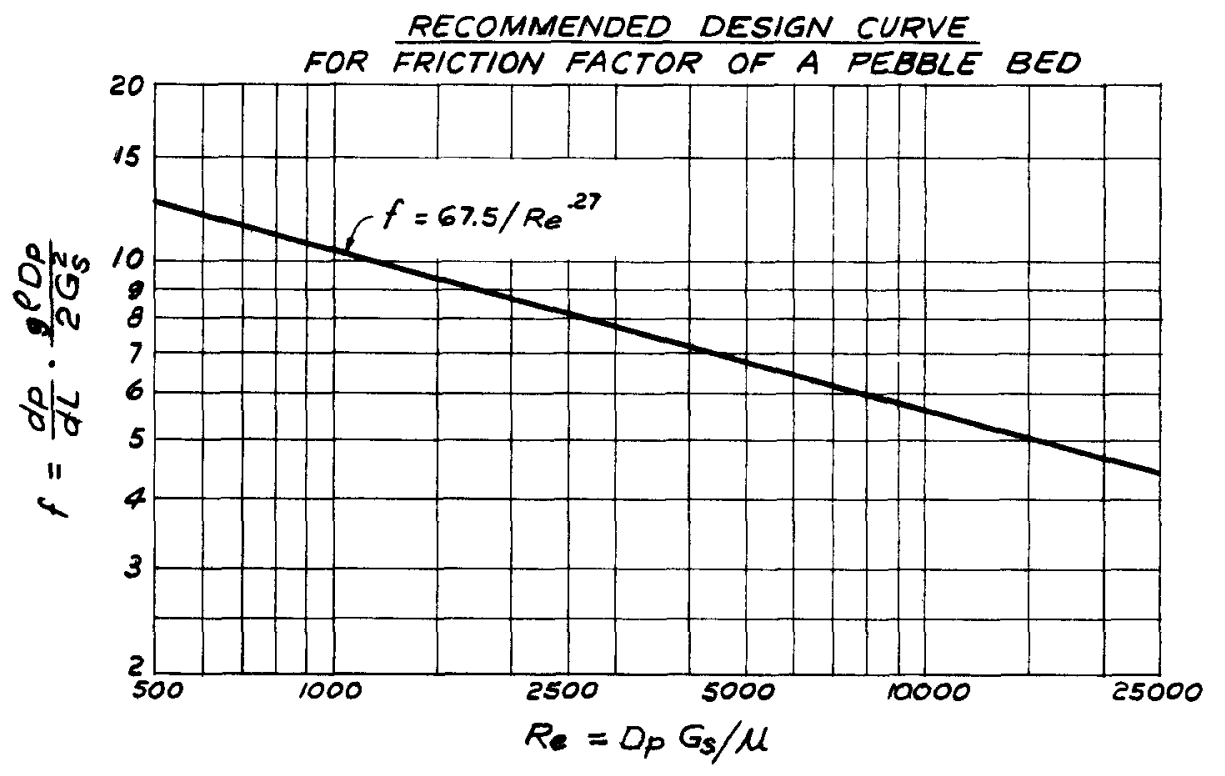

$$
2-10
$$


interest and therefore was not included. Using the upper limit of the mean tolerance curve appears conservative since it embraces both the data of Denton and Rose. When data is available on friction factors at high Reynolds numbers and within our range of interest, these data will be re-evaluated.

In considering the flow through an infinite pebble bed, it is obvious that there are not uniform, continuous flow lines. The bed is composed of a series of interconnected voids of different size and the fluid passes from one void to another in its passage through the bed. Carman (2) postulates that instead of the velocity increasing and decreasing from void to void, it is nearer to the true state to assume that it is constant. Within the bed there cannot be any isolated channels since the whole system of voids is interconnected, so that where the cross-section or volume of one void is decreasing in the direction of flow, the velocity does not increase but the fluid stream moves to a neighboring void, the section of which is enlarging in the direction of flow. It is apparent that every flow line of the fluid, in the continual division and rejunction with other flow lines, must follow a very tortuous path. The true picture is one of an element of fluid winding its way along a path through a bed, changing direction at random from point to point, but with a net vector perpendicular to the face of the bed.

When we consider the flow through the total bed we get a slightly different picture. Assuming a uniform velocity profile at the face of the bed, we would expect to get a velocity profile at a few ball diameters deep which was a function of the voidage. The velocity would be largest near the wall, leveling off to a smaller velocity in the characteristic section. However, as flow progresses through the bed there will be an increase in the radial component of velocity with more and more fluid moving towards the wall until, at the exit, the wall velocity is appreciably greater than that at the center. This is illustrated in Figure $2.1=7$.

Data on this velocity profile is scattered and varies over a considm erable range. Since it is a function of the voidage, we have chosen to use a characteristic voidage of $38 \%$ and algebraically determine the wall voidage (extending over a range of $2 D_{p}$ ) that will give a bed voidage of $39 \%$.

When using voidages other than $39 \%$, equation (2.1-13) is rewritten as:

$$
f=7.5{\frac{(1-\varepsilon)}{\varepsilon^{3}}}^{1.27} / \mathrm{Re}^{.27}
$$


FIG. $2.1-7$

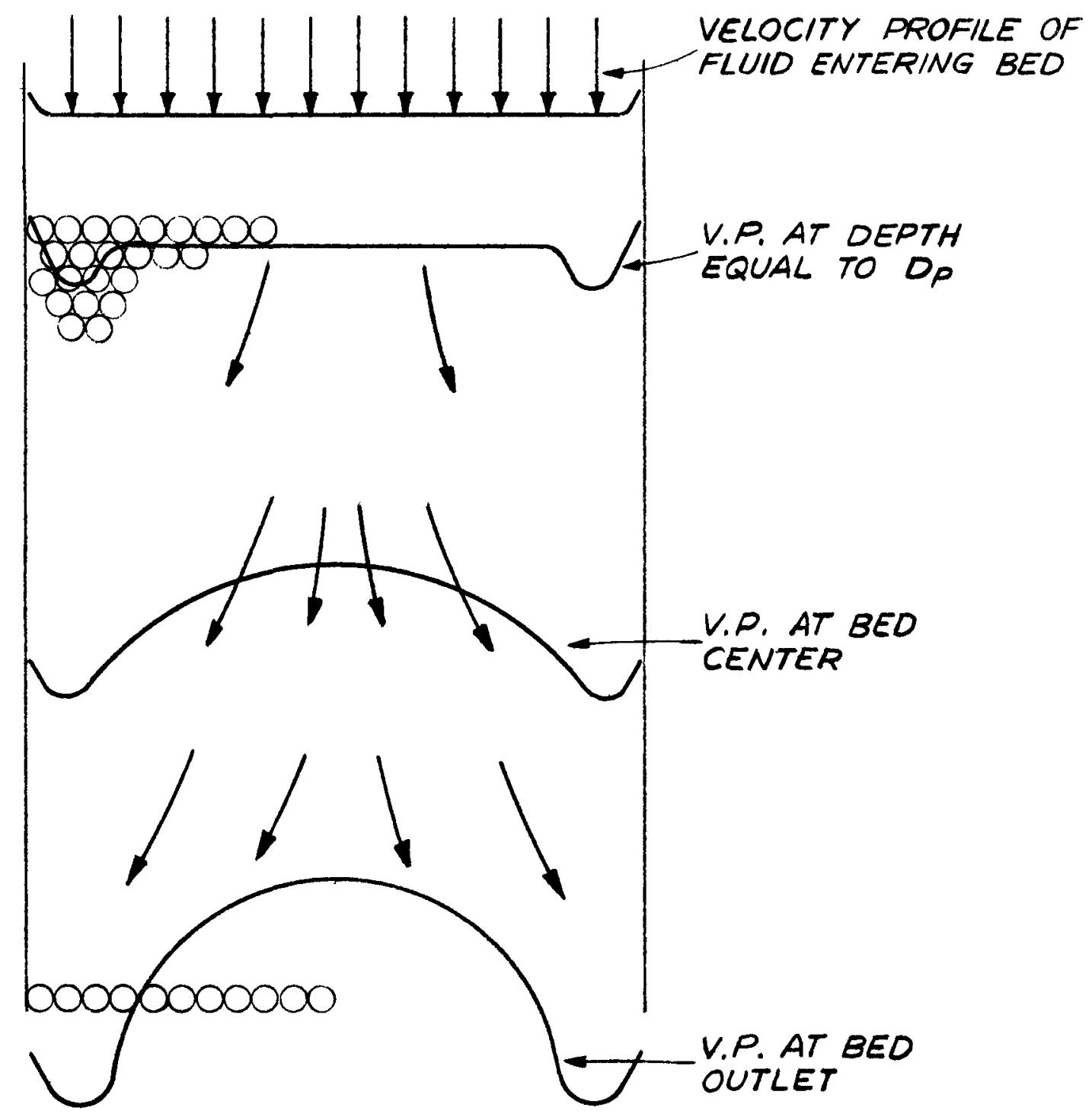

\section{POSTULATED VELOCITY PROFHES \\ THRU A PEBBLE BED (ISOTHERMAL CASE)}




\section{1.4 Thermal Characteristics of Randomly Packed Beds}

Thermal characteristics of packed beds of spheres are correlated by plotting the Colburn heat transfer grouping, $j$, vs. a modified Reynolds number, $R e^{\prime}$, where:

$$
\begin{aligned}
& j=h P_{r} \cdot 66 / G_{s} C_{p} \\
& R e^{\prime}=D_{p} G_{s} / \mu(1-\varepsilon)
\end{aligned}
$$

Figure 2. 1-8 presents a plot of heat transfer grouping $j$ vs $R^{\prime}$ for heat transfer tests on beds of spheres as conducted by a number of investigators, Kaye and London (5), Denton, Robinson \& Tibbs (6) Glasser and Thodos (7) and Gamson (13). Three of these runs were made concurrent with the friction tests reported in Section 2.1-3. These results also have a spread of $\pm 25 \%$. In order to be on the conservative side, a value of $75 \%$ of the mean is used in design. This results in the following expression for, $j$, :

$$
\mathrm{j}=0.5 / \mathrm{Re}^{10.3}
$$

Since our survey work will be based on beds with a uniform voidage of $39 \%$, this value is substituted in equations $(2.1-18)$ and $(2.1-19)$ to give:

$$
\mathrm{j}=.43 / \mathrm{Re}^{0.3}
$$

where $\quad R e=D_{p} G_{s} / \mu$

Equation (2. 1-20) is the relationship used for design and it is plotted in Figure 2. 1-9.

Comparing our design curve with the data of Kaye and London (5), the mean data of Figure 2. 1-8 lies on the Kaye and London correlation line with a slightly lower slope. Since there is little data reported in the area of Reynolds numbers where we expect to operate these data are adequate until other material becomes available. 
FIG. $2.1-8$

HEAT TRANSFER CORRELATION FOR PEBBLE BEDS

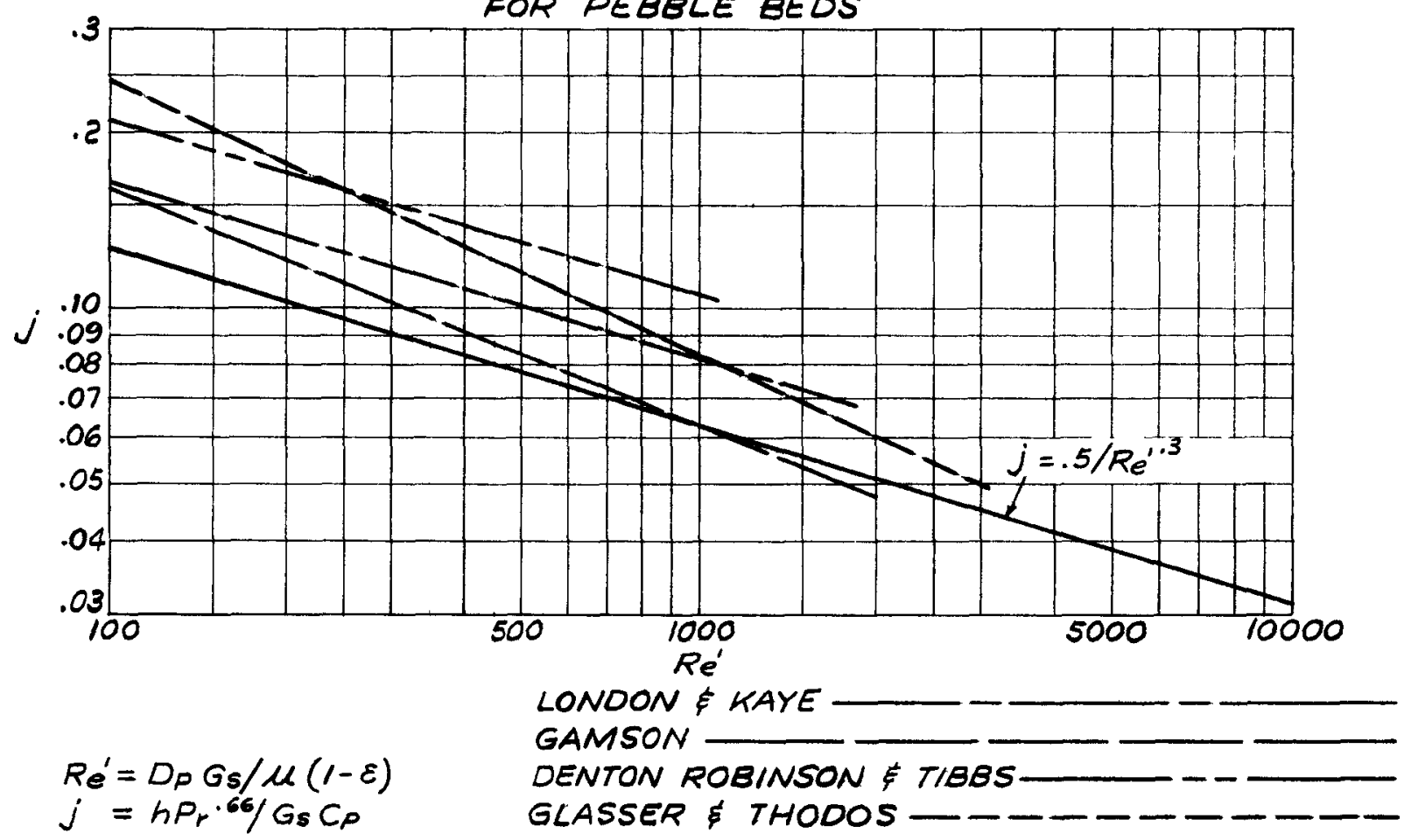

FIG. 2. $1-9$

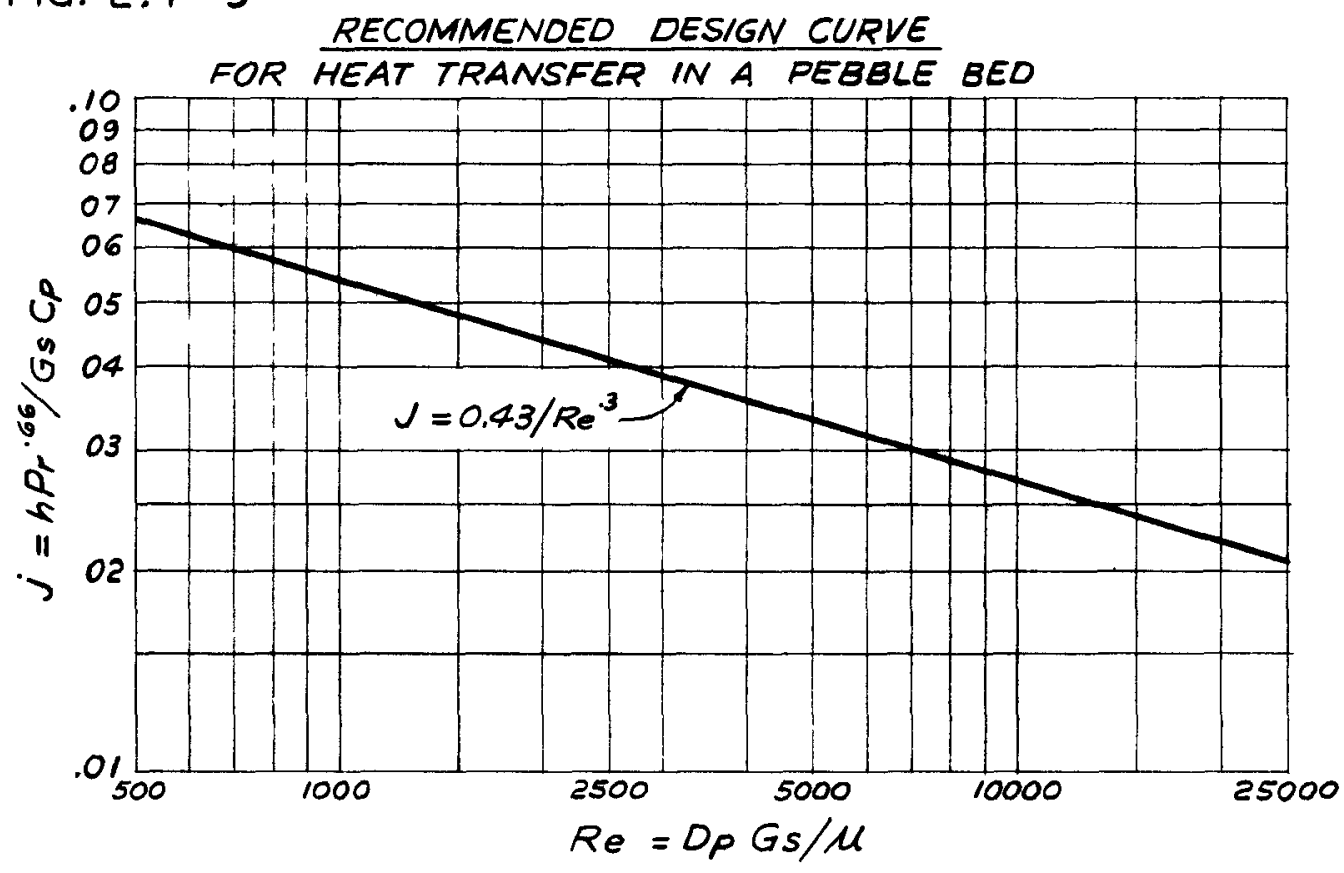

$2-14$ 


\section{1.5 Application of Pebble Beds to Nuclear Reactors}

There have been a number of studies made of the use of spherical fuel elements in nuclear reacturs (Ref. 15 to 20). These studies have considered both ceramic and metallic clad spheres, and both liquids and gases as coolants. The pattern that has developed is that where liquids are used as coolants the bed is fluidized, and where gases are used as; coolants the bed is fixed. The use od a fluidized bed where a liquid is used as a coolant appears to be necessary to eliminate hot spots on the ball surface that would cause local boiling. Fluidized bed reactor cores are feasible using liquid coolants because of the stable nature of such beds. This condition is not present with a gas. It is virtually impossible to fluidize a reactor core using a gas stream since slugging, rat-holing and otier such unstable phenomena result in an uncertain and variable vuid fraction in the core. In addition since boiling is not a problem with a gaseous coolant, the aforementioned objection to a fixed bed has no significance. It is necessary, however, to avoid metallic coatings on the fuel elements to prevent local welding due to hot spots.

There are a number of characteristics of pebble beds that make their use attractive in a gas-cooled, ceramic reactor. Principal among them are the ease with which the fual can be handled, the excellent structural characteristics of the spherical shape and the potential economies in its fabrication.

From the standpoint of fuel handling, a pebble bed reactor can be loaded and unloaded by gravity, taking advantage of the flow characteristics of spherical particles. What this means in equipment is flasks to hold fresh and spent fuel elements with appropriate locks and valves, and pipes leading to and from the reactor.

From the standpoint of manufacturing, spheres can be fabricated in a number of ways, which can be readily adapted to mass production, thus lowering the costs. In a fixed pebble bed reactor many thousands of spheres will be randomly packed in the reactor core, thus individual variations from one fuel element to the next will have no significant effect on the core properties. This means that close tolerances of size and shape are not required, which should ease the fuel element manufacturing problems considerably.

With a ceramic or refractory matrix, which also serves as the moderator, the temperature limits normally associated with a reactor design assume different proportions. Graphite, unlike other materials, 
actually exhibits an increase in strength with increasing temperature. Increasing temperature cannot aggravate corrosion problems because none exist when an inert gas is used as the coolant. By accepting the presence of radioactivity in the primary loop, problems associated with increased leakage of gaseous fission products through cladding at increasing temperatures are not of primary interest.

The amount of heat transfer surface per unit of bed volume is not large for the range of ball size under consideration. It is independently controllable and can be made as large or small as is desired by simply varying the ball size. There are practical limitations, however, which are imposed by the increased $\Delta P$ which accompanies decreased ball size. In the range of size presently under consideration, i.e. $1 / 2^{11}$ balls to $1-1 / 2^{\prime \prime}$ balls, the surface varies from about $87 \mathrm{ft}^{2} / \mathrm{ft}^{3}$ to $29 \mathrm{ft}^{2} / \mathrm{ft}^{3}$. These values result in film temperature drops as high as $360^{\circ} \mathrm{F}$ which tends to increase the fuel element surface temperature somewhat, however no problem exists since the surface temperatures are still far below the limits for our structural materials, even in the most perverse case.

As with all gas cycle reactors the ratio of pumping power to heat transfer is somewhat higher than for a liquid-cooled reactor. On the other hand, the high outlet temperature from a ceramic gas-cooled reactor means a high power plant efficiency resulting in a greater net plant output for a given amount of reactor heat. The friction factor of a pebble bed is high compared to a reactor with continuous coolant channels, necessitating a larger face area for the pebble bed to keep an equivalent pumping power.

One rather unique feature of a pebble bed reactor core, which results from the fact that all voids are interconnected, is that either axial or radial flow may be had with equal ease. This enables the designer to select the flow pattern which is best for each particular application.

In any practical reactor core the local rate of heat generation shows appreciable variations in a radial and axial direction. The effect of this non-uniform heat generation on the temperature-dependent properties of gas density and viscosity are such as to divert flow away from the high flux regions. This accentuates the non-uniform temperature profile, which in turn aggravates the maldistribution of flow. This situation is particularly bad in a single region core where the peak radial flux is on the bed centerline where voidage is least and the minimum flux is at the wall where voidage is the greatest. 
The nuclear survey of pebble beds has shown that in order to achieve a high conversion ratio it is necessary to reduce the voidage of the bed from that of $39 \%$ for the finite bed to something between 20 and $25 \%$ by dispersing extra graphite over the bed face. One way of doing this is to place the fuel in channels which results in flux peaking in the wall between channels with minimum flux at the channel centerline, e.g. the characteristic of self-shielding of a fuel cluster. Superimposed upon this effect is the overall effect of flux buckling due to neutron leakage, resulting in a radial power generation similar to that of the reference core and shown in Figure 2.4-1. Thus we have a reverse of the condition that existed in the single region bed, $i$.e. the point of maximum flux coincides with that of maximum voidage and vice versa.

The distribution of flow through such a core can be approximated, based on the fact that the pressure drop across all stream filaments must be equal, together with a knowledge of distribution of voidage in the bed. The weight flow in any stream filament varies inversely with a power of the average bulk gas temperature and directly with a power of the local voidage. The combined effect of flux and voidage distribution in a fuel channel is such as to virtually eliminate the high ratios of max.* imum to minimum temperature as found in the single region bed. 


\section{2 Thermal and Hydraulic Analysis of Pebble Beds}

A nuclear analysis was conducted covering a range of pebble beds from seven to twenty feet in diameter. Core characteristics affecting the fluid dynamic and thermal characteristics were the diameters and voidage. The voidage of a finite bed of randomly packed spheres is $39 \%$ which is the case in a single region core. To increase the carbon to fuel ratio over that obtainable in a single region core, when the weight percent of fuel is held a constant, it is necessary to assume that carbon is "smeared" throughout the core in, for example, islands. This reduces the voidage with respect to core diameter, although the actual ball bed voidage remains at $39 \%$.

From equation $2.1-14$

$$
\Delta P=\frac{2 f G_{S}^{2} L}{g \rho D_{p}}
$$

For a randomly packed bed of $39 \%$ voidage

$$
\begin{aligned}
& f=\frac{d p}{d L} \frac{g \rho D_{p}}{2 G_{s}}=67.5 / R e^{11.27} \\
& R e=D_{p} G_{s} / \mu \\
& G_{s}=W / A_{s} \\
& L=V / A_{s}
\end{aligned}
$$

Expressing $D_{p}$ in inches and $\mu$ in lbs/Ft/Hr

$$
\Delta P=\frac{10.75 W^{1.75} \mu \cdot 27 V}{\rho D_{p}^{1.27} A_{s}^{2.73}}
$$

$\mu$ can be expressed as $.00131 \mathrm{~T} \cdot 58$ over the range of temperature of interest. $p$ is.equal to $.373 \mathrm{P} / \mathrm{T}$ for helium where $\mathrm{P}$ is in psia.

Substituting in $2.2-1$

$$
\Delta \mathrm{P}=\frac{4.78 \mathrm{~W}^{1.73} \mathrm{~T}^{1.157 \mathrm{~V}}}{\mathrm{P} \mathrm{D}_{\mathrm{p}}^{1.27} \mathrm{As}^{2.73}}
$$


The face area of the bed, $A_{S}$, can be expressed in terms of the volume V by:

$$
A_{S}=V^{2 / 3}(\pi / 4)^{1 / 3}(D / L)^{2 / 3}=0.9225\left(\frac{V}{L / D}\right)^{2 / 3}(2.2-3)
$$

For a core having an L/D of 0.9

$$
A_{S}=0.99 \mathrm{~V}^{2 . / 3} \& A_{s}^{2.73}=0.973 \mathrm{~V}^{1.82}
$$

Substituting in $2.2-2$

$$
\Delta \mathrm{P}=\frac{4.92 \mathrm{~W}^{1: 73} \mathrm{~T}^{1.157}}{\mathrm{P} \mathrm{D}_{\mathrm{p}}^{1.27} \mathrm{~V}^{.82}}
$$

Initial design considerations set the reactor inlet temperature at $500^{\circ} \mathrm{F}$ and the outlet temperature at $1200^{\circ} \mathrm{F}$, resulting in a temperature rise through the core of $700^{\circ} \mathrm{F}$. The design rating of $350 \mathrm{tMW}$ of reactor heat results in a helium flow of $378 \mathrm{lbs} / \mathrm{sec}$. System pressure is 1000 psia.

Substituting these values in $2.2-5$

$$
\Delta P=\frac{577,000}{D_{p}^{1.27} \mathrm{~V}^{.82}}
$$

The blower head required to overcome the core pressure drop is equal to:

$$
\begin{aligned}
& \mathrm{H}_{\mathrm{ad}}=\Delta \mathrm{P}_{\mathrm{r}} / \rho_{\mathrm{p}} \text { in Ft. } \\
& \rho_{\mathrm{p}}=.373 \times 1000 / 960 \text { Lbs } / \mathrm{Ft}^{3} \\
& \mathrm{H}_{\mathrm{ad} p}=2.57 \Delta \mathrm{P}_{\mathrm{r}} \text { in Ft } \\
& \mathrm{w}_{\mathrm{p}}=\mathrm{H}_{\mathrm{ad}} \mathrm{W} / 738 \text { in } \mathrm{KW} \\
& \mathrm{wp}=2.57 \Delta \mathrm{P}_{\mathrm{r}} 378 / 738=1.315 \Delta \mathrm{P}_{\mathrm{r}}
\end{aligned}
$$

Let $w / y=\beta$ the ratio of pumping power to heat transferred

$$
\beta=\frac{1.315 \Delta P_{r}}{350,000}=\frac{\Delta P r}{266,000}-
$$


Thus a $\Delta \mathrm{P}$ through the core of 2660 psf represents $1 \%$ of the heat trans ferred in pumping power.

Substituting in $2.2-6$

$$
\beta=2.17 / \mathrm{D}_{\mathrm{p}}^{1.27} \mathrm{~V}^{.82}
$$

This relation was solved over a range of values of $\beta$ from 1 to $5 \%$ and core volumes from 100 to $10,000 \mathrm{Ft}^{3}$ by rearranging and solving for $D_{p}$

$$
D_{p}=1.835 / \beta^{.7 \varepsilon 7} \mathrm{~V}^{.645}
$$

These results, applicable to a single region core of $39 \%$ voidage, are presented in Figure 2.2-1.

As a result of the nuclear survey reported in Section 1 of Part II it was determined that in order to approach or achieve a net breeding gain in a pebble bed reactor it would be necessary to add additional carbon to the core which is expressed by specifying a voidage less than $39 \%$. For example, adding $25 \%$ carbon to the core would be expressed as a voi.dage of $(1-.25) .39=29.3 \%$. As a result of the nuclear survey voidages between 20 and $30 \%$ appeared most likely to satisfy the design conditions, so the fluid friction characteristics of cores of these void=. ages were determined.

It was also apparent from the nuclear survey that all cores would be two region or be blanketed. Since at this time the characteristics of the blanket as a function of core diameter were not known and it was not known precisely how flow would be handled through the blanket, its effect on the thermal and hydraulic characteristics was neglected.

Voidages of 30 and $20 \%$ result in a ball bed area in the core of 76.9 and $51.3 \%$ of the face area respectively. From the standpoint of the friction characteristics, this changes the $L / D$ of the bed as follows:

$\%$ Voidage

L/D
39

0.9
30

1.026
20

1. 257

Substituting these values in $2.2-4$
$\%$ Voidage
39
$\mathrm{As}_{\mathrm{s}_{2}: 73}$
$0.99 \mathrm{~V}^{2 / 3}$
30
20
$0.907 \mathrm{~V}^{2 / 3}$
$0.766 \mathrm{~V}^{1.82}$
$0.792 \mathrm{~V} 2 / 3$
$0.973 \mathrm{~V}^{1.82}$
$0.528 \mathrm{~V}^{1.82}$ 


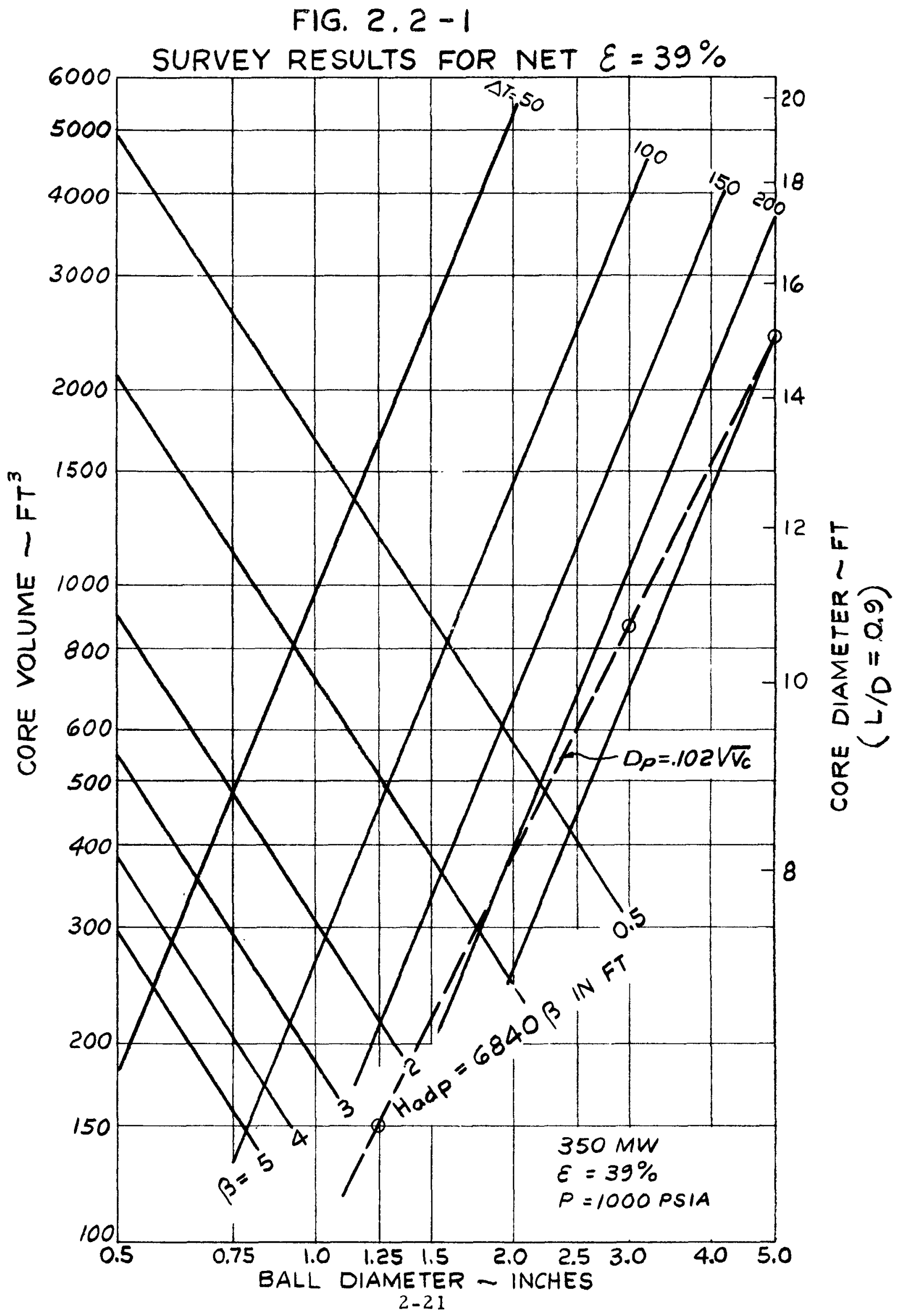


FIG. 2. 2-2

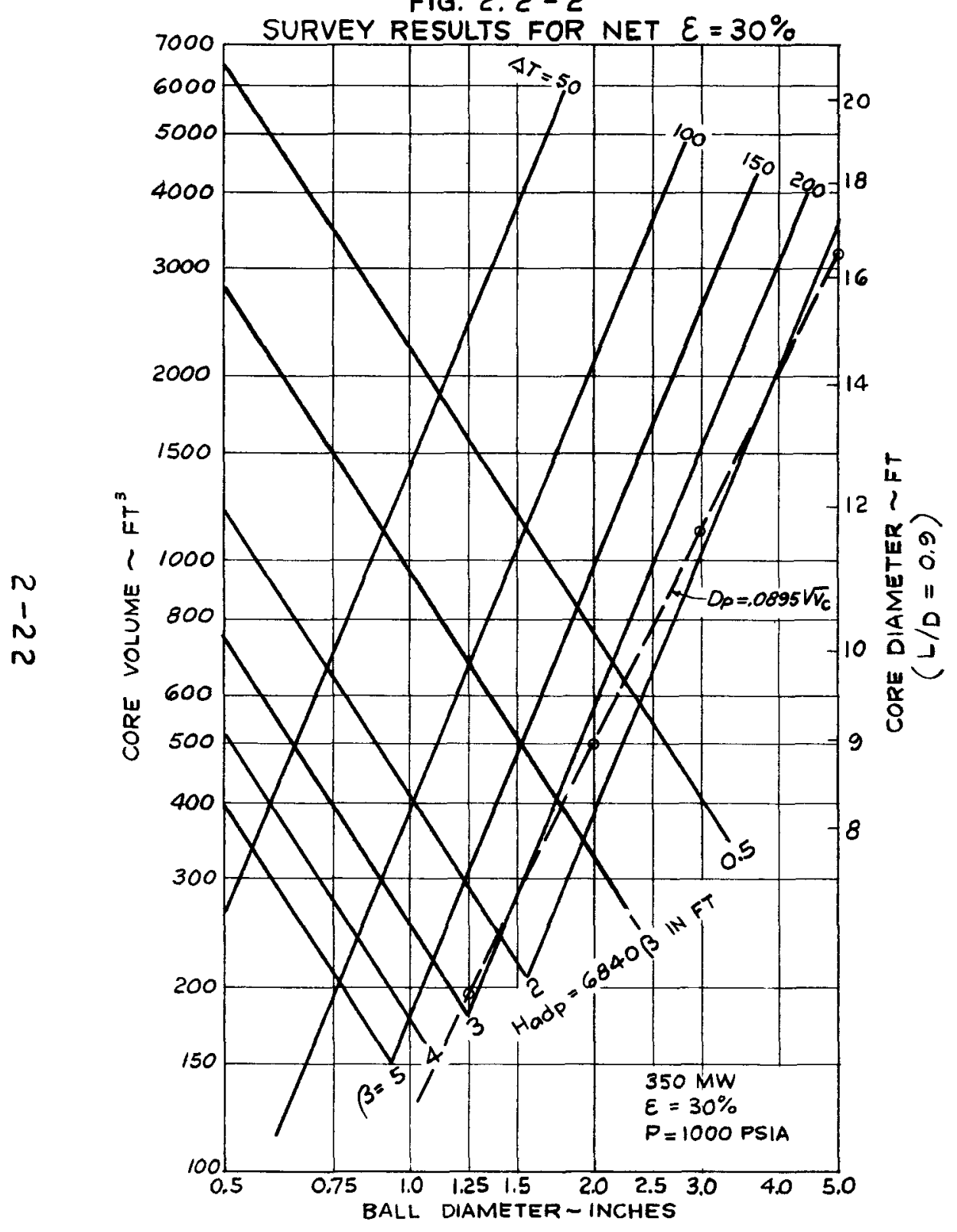

FIG. 2. 2-3

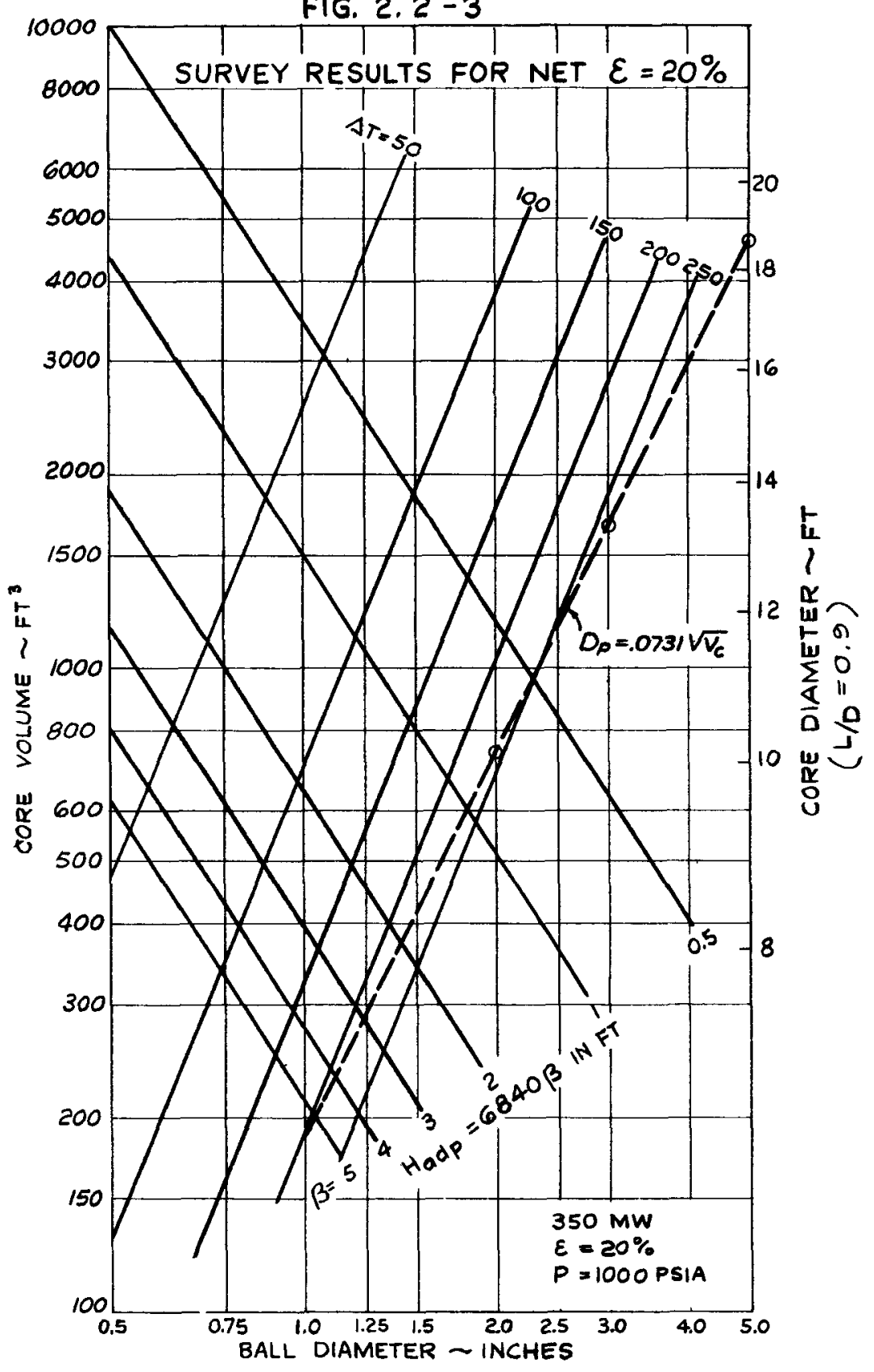




$$
\begin{array}{ll}
\beta=2.17 / \mathrm{D}_{\mathrm{P}} 1.27 \mathrm{~V}_{\mathrm{V}} \cdot 82 & \text { for } \varepsilon=35 \% \\
\beta=2.76 / \mathrm{D}_{\mathrm{P}} 1.27 \mathrm{~V}_{\mathrm{V}} .82 & \text { for } \varepsilon=30 \% \\
\beta=4.0 / \mathrm{D}_{\mathrm{p}} 1.27 \mathrm{~V}^{.82} & \text { for } \varepsilon=20 \%
\end{array}
$$

Pumping power fractions for cores of 30 and $20 \%$ voidage were determined for the same volume range of 100 to $10,000 \mathrm{Ft}^{3}$ and the results presented in Figures $2.2-2$ and 2.2-3 respectively.

The weight flow used in determining the pressure drop characteristics of the cores is such as to yield a temperature rise tinrough the coro of $700^{\circ} \mathrm{F}$. To this can be added the temperature drop through the film to get an idea of the order of magnitude of the fuel element surface temperatures.

$$
h=j G_{s} C_{p} / P_{r} 2 / 3
$$

From equation 2. 1-20

$$
\mathrm{j}=0.43 / \mathrm{Re}^{\cdot 3}=0.43 \mu \cdot 3 / \mathrm{D}_{\mathrm{p}} \cdot 3 \mathrm{G}_{\mathrm{s}} \cdot 3
$$

substituting in equation $2.2-11$

$$
h=0.43 \mu \cdot 3 G_{s} \cdot 7 C_{p} / D_{p} \cdot 3 \operatorname{Pr} 2 / 3
$$

Expressing $h$ in $B T U / H r / F t 2 /{ }^{\circ} F, \quad D_{p}$ is in inches and $G_{s}$ in $1 \mathrm{bs} / \mathrm{sec} / \mathrm{Ft}^{2}$

$$
\begin{aligned}
& h=281 \mu \cdot 3 G_{s}: 7 C_{p} / D_{p} \cdot 3 \operatorname{Pr}^{2 / 3} \\
& G_{s}=W / A_{s}
\end{aligned}
$$

and fur $39 \%$ voidage

$$
A_{S}=0.99 V^{2 / 3} \text { and } A_{s} \cdot 7=.993 \mathrm{~V} \cdot 467
$$

subsituting in equation 2.2-13

$$
\mathrm{h}=283 \mathrm{~W} \cdot 7 \mathrm{C}_{\mathrm{p}}{ }^{\cdot 3} / \mathrm{D}_{\mathrm{p}} \cdot 3 \mathrm{~V}^{\cdot 467} \mathrm{P}_{\mathrm{r}} 2 / 3
$$


Design conditions are:

$$
\begin{array}{ll}
W=378 \# / \mathrm{sec} & \mathrm{W}^{\cdot 7}=64 \\
\mathrm{~T}_{\mathrm{m}}=850^{\circ} \mathrm{F}=1310^{\circ} \mathrm{R} & \\
\mathrm{C}_{\mathrm{p}}=1.25 & \\
\mu=.084 \mathrm{bs} / \mathrm{Hr} / \mathrm{Ft} & \mu \cdot 3 \cdot \overline{3}=0.476 \\
\mathrm{P}_{\mathrm{r}}=0.673 & \mathrm{Pr}_{\mathrm{r}} 2 / 3=0.768 \\
\mathrm{~h}=14,000 / \mathrm{D}_{\mathrm{p}} \cdot 3 \mathrm{~V} \cdot 467 &
\end{array}
$$

The $\Delta \mathrm{T}$ film is equal to the rate in $\mathrm{BTU} / \mathrm{Hr} / \mathrm{Ft}^{2}$ divided by the film coefficient.

$$
\begin{aligned}
& \Delta T_{f}=\frac{Q}{S_{h}}=\frac{P 3415}{S_{h}} \\
& P=350 \mathrm{MW} \\
& S=43.92 \mathrm{~V} / D_{p_{3}} \mathrm{~V} \cdot 467 \\
& \mathrm{~h}=14,000 / D_{\mathrm{p}}{ }^{3} \mathrm{~V} \\
& \Delta \mathrm{T}_{\mathrm{f}}=1940 \mathrm{D}_{\mathrm{p}} 1.3 / \mathrm{V}^{.533}
\end{aligned}
$$

$\Delta T_{f}$ was determined for a range of cores of volumes from 100 to $10,000 \mathrm{Ft}^{3}$ and voidages of 39,30 and $20 \%$ and the results plotted on the pumping power curves of Figures 2.2-1, 2.2-2 and 2.2-3.

As is shown in Section 2.3, a relation was established between the volume of the core and the maximum ball diameter, based on thermal stress limitations. This relation is equal to:

$$
D_{p}=0.102\left(V_{b}\right)^{5}
$$

for a core of 350 tMW output and is plotted on Figures 2.2-1,-2 and -3 . Acceptable combinations of core size and ball diameter lie above this line.

These generalized plots are based on a system pressure level of 1000 psia. $\beta$ for pressures other than 1000 can be obtained by multiplying the value of $\beta$ at 1000 by $(1000 / P)^{2}$.

The nuclear survey has shown that in order to get a high conversion. ratio, or in the case of large cores, a net breeding gain, it is necessary to design with a voidage of between 20 and $30 \%$. Figure $2.2-4$ presents a 
FIG $2 \cdot 2-4$

Core Characteristics vs. $\beta$ \& I BR 350 t MW- $700^{\circ} F$ St - 1 1/4 IN BALLS

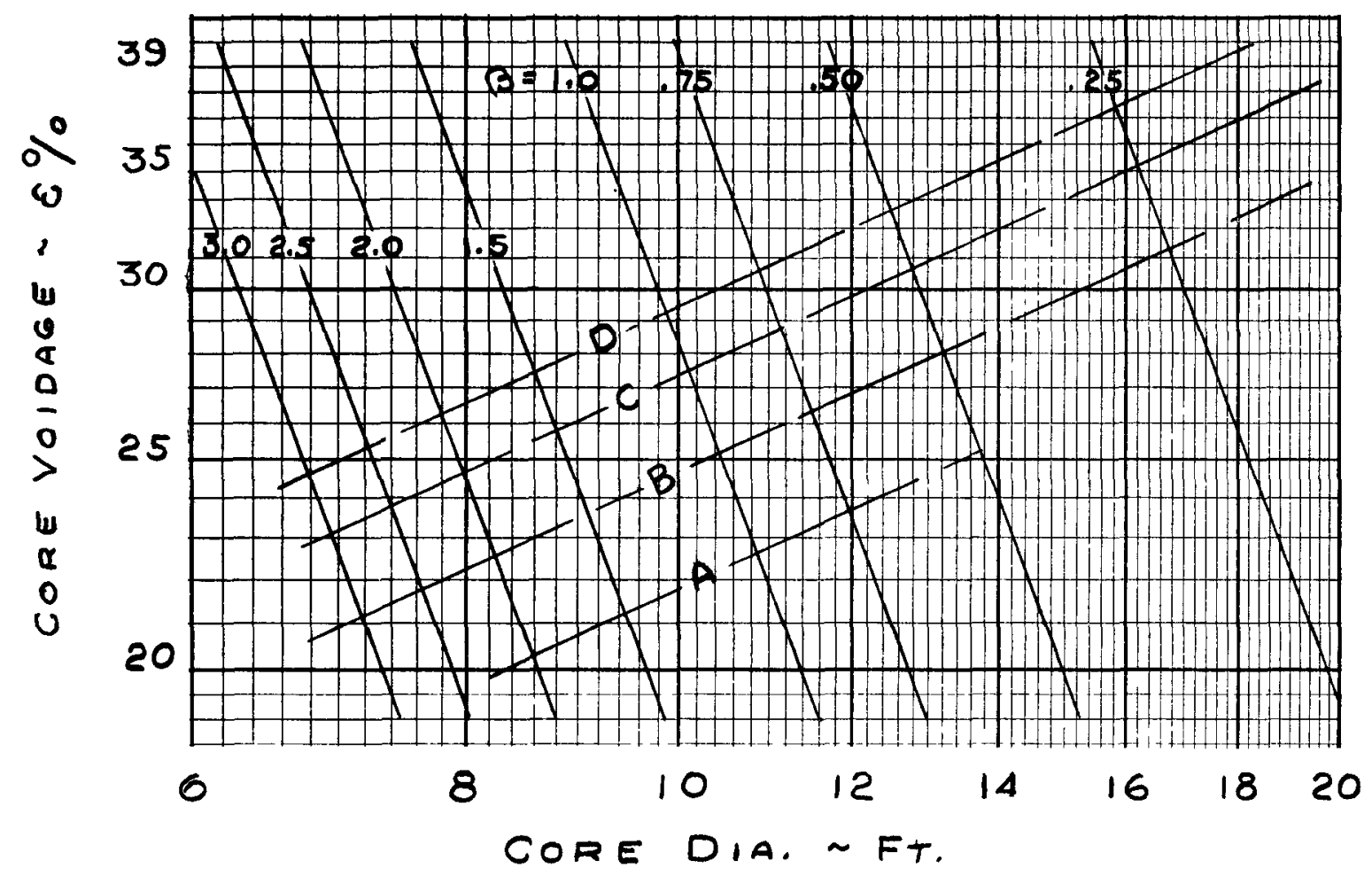

$\begin{array}{ccc}\text { CORES } & \text { Ko SF } & \text { I BR } \\ A & 1.10 & 0.90 \\ B & 1.10 & 0.85 \\ C & 1.00 & 1.00 \\ D & 1.00 & 0.95\end{array}$

(REF SECT, 1.2.7)

$2-25$ 
plot of voidage $\varepsilon$ vs. core diameter with lines of constant $\beta$, using a 1-1/4" ball as the parameter. Cores having selected nuclear parameters, such IBR and $\mathrm{K}_{\text {eff }}$ can be cross-plotted on a curve of this type to evaluate the relative worth of different possible cores from the standpoint of pumping power.

From the standpoint of net plant output and selection of blowers to circulate the helium it is desirable that the core pumping power be between 1 and $1-1 / 2 \%$ of the heat output. This would narrow a selection of cores to those lying between 7.6 to 9 feet in diameter with $39 \%$ voidage and 9.6 to 11.4 feet in diameter with $20 \%$ voidage. 


\section{3 Selection of Fuel Element Size}

As shown in Section 2.2, both the thermal and hydraulic characteristics of the reactor core are affected by ball diameter. A larger ball diameter will decrease the core pressure loss but will increase the fuel element surface temperature. However, since the pressure loss reduction tends to be more significant than the increased surface temperatures in the range of conditions we have studied, it is of interest to find the maximum allowable ball diameter for a given size core. Ball size also has an effect on the size of ball handling passageways (larger balls need larger passageways in order to avoid bridging or plugging) and an effect on fuel fabrication and reprocessing charges since larger balls mean fewer fuel elements to be handled for a given core bed volume and ball price per pound decrease with increasing size.

The most important factor which limits the maximum ball size will be the tangential tensile stress at the outer surface of the ball caused by the internal generation of heat. For uniform heat generation within the ball, thermal stress depends on a number of factors as shown by the following relationship:

$$
\sigma_{t}=a E^{2} / 8640 k(1-v)
$$

where $\sigma_{t}=$ tangential stress at outer surface, psi

$\mathrm{Q}^{\mathrm{t}}=$ volumetric heat generation rate, $\mathrm{BTU} / \mathrm{Hr}-\mathrm{Ft}^{3}$

$\mathrm{D}=$ ball diameter, inches

$a=$ coefficient of thermal expansion, in $/$ in $-{ }^{\circ} \mathrm{F}$

$\mathrm{E}=$ modulus of elasticity, psi

$\nu=$ Poisson's ratio

$\mathrm{k}=$ thermal conductivity, $\mathrm{BTU} / \mathrm{Hr}-{ }^{\circ} \mathrm{F}-\mathrm{Ft}$

In other words, for a given volumetric heat generation, the maximum allowable fuel element diameter is a function of five properties of the fuel elements: $\sigma, a, E, v$ and $k$. A review of the literature pertaining to these properties of graphite and uranium-graphite shows a wide variation which is primarily due to the variety of raw materials ard manufacturing methods. In addition, these properties are a function of both temperature, radiation exposure and, in the case of uranium-graphite, the method used to add uranium to the graphite.

Figure 2.3-1 is plot of tensile stress, modulus of elasiticity and coefficient of thermal expansion of unirradiated graphite as a function of 
FIG, 2. 3-I

SOME PROPERTIES OF GRAPHITE AS A FUNCTION OF TEMPERATURE

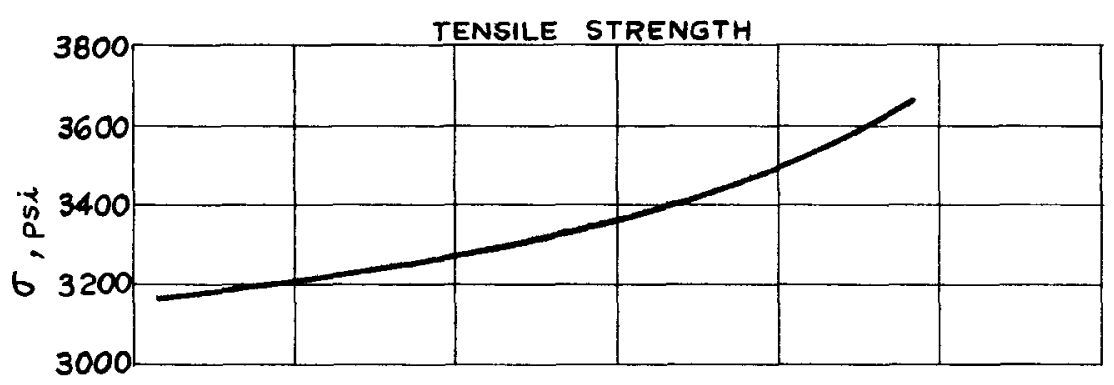

N

in

$\infty$
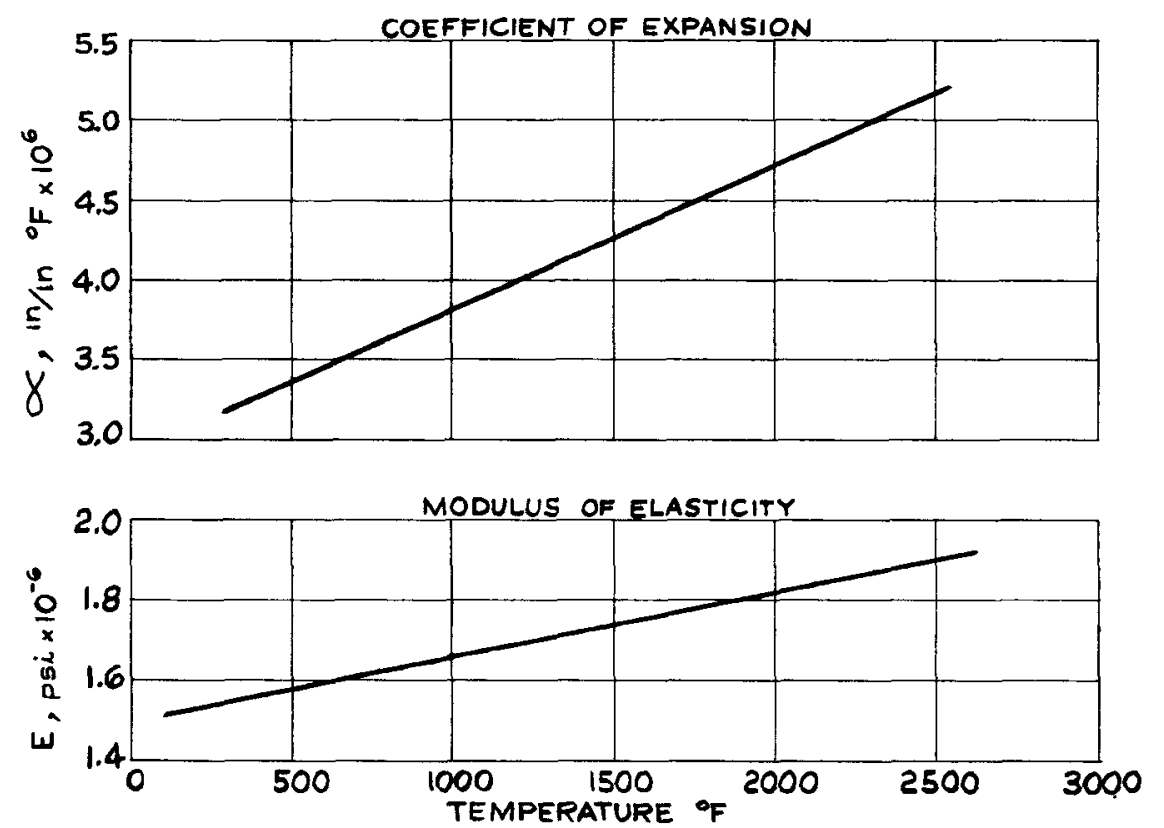

FIG. 2. 3-2

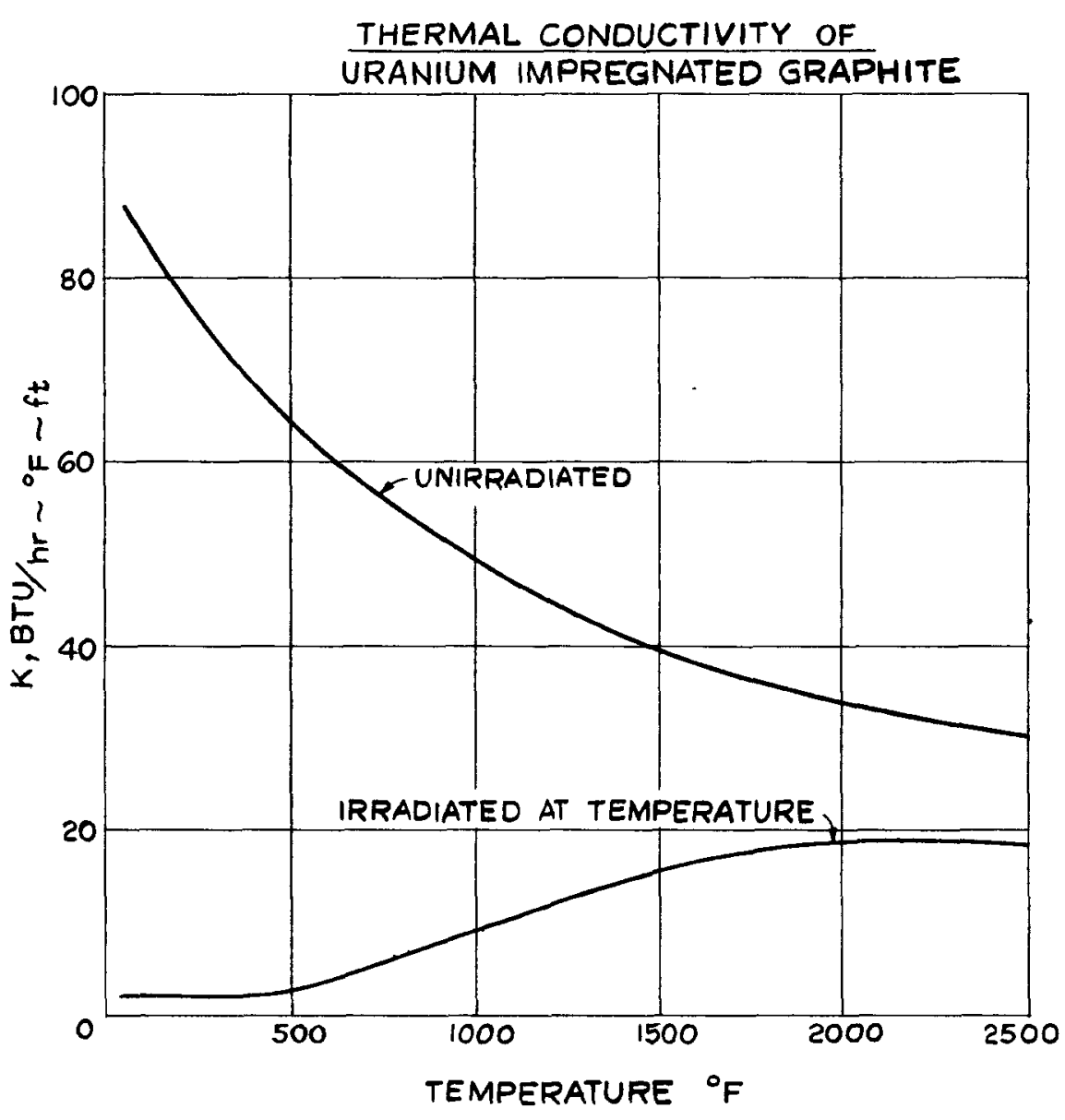


temperature. Figure 2.3-2 is a similar plot of thermal conductivity. These values, typical of what can be obtained for the molded 1.7 density graphite used in the PBR, are taken from the references $(21)$ and $(22)$, and have been used as the basis for this study. The presence of $\mathrm{UO}_{2}$ and $\mathrm{ThO}_{2}$ has been assumed to have no affect on these properties in the unirradiated state since they will be added by impregnating the pores of the graphite. A further correction must be made, however, for the effect of irradiation.

In general, the effect of irradiation on graphite is to change it from a soft material with a well-ordered lattice structure to a relatively hard material with a disordered lattice structure. The increased hardness is associated with an increase in both strength and modulus of elas ticity. The disordered lattice structure has the effect of decreasing the thermal conductivity. The temperature at which irradiation occurs has a strong influence on the magnitude of these effects. Much work has been done on fast neutron damage to graphite at low temperatures in reference to graphite piles for research and production. While the magnitude of the damage can be severe, much of the fast neutron damage can be removed by annealing the graphite at higher temferature.

In the case where uranium is in intimate contact with the graphite, the bulk of the damage is caused by fission fragments rather than fast neutrons. The extent of this fission fragment damage is inversely proportional to the size of lumps in which the uranium is incorporated within the graphite as shown in reference (23). A lumped uranium-graphite fuel element can be made by incorporating $\mathrm{UO}_{2}$ particles with the graphite during its fabrication. Although it is expected that uranium impregnated graphite will suffer relatively more damage than lumped uranium graphite, we have chosen the former type of fuel element for other reasons as discussed in S\&P 1963, Part I.

Thermal conductivity is the property of interest that is most affected by radiation change. Work by'North American Aviation (24) on uranium impregnated graphite has shown that the decrease in thermal conductivity with ir radiation is strongly affected by the temperature at which the specimen is irradiated. At low temperature (up to $100^{\circ} \mathrm{C}$ ), a decrease by a factor of 30 is noted while at high temperature $\left(1000^{\circ} \mathrm{C}\right)$, a decrease of the order of only a factor of 2 is reported. One run of interest at $730^{\circ} \mathrm{C}$, comparable to the operating temperature of the fuel element located at the point of maximum flux in the PBR, showed a decrease by a factor of about 2.5. This factor was considerably smaller 
than that reported in early work at ORNL (25). However, as pointed out by NAA, their own data did show a saturation of the "decrease factor" with exposure time. These thermal conductivity decrease factors for various temperatures of irradiation are applied to the thermal conductivities shown in Figure 2.2-3, and plotted thereon.

In using these values of thermal conductivity, it should be realized that our fuel element exposures will exceed those of existing experimental data. If further experimental work should indicate that irradiation damage would be excessive for our present reference fuel element, the use of the lumped uranium graphite fuel element would be considered.

A relationship between core volume and fuel element diameter was established for use in the parameter survey. For this purpose, the maximum-to-average power density was set at 2.0 and all graphite properties were taken at $1500^{\circ} \mathrm{F}$ to correspond with a fuel element at the maximum flux conditions. Since increases in tensile strength and modulus of elasticity with irradiation are of the same order, this effect has been omitted because it cancels out in equation (2.3-1). No irradiation effect is included with the coefficient of expansion and Poisson's ratio is assumed constant $(v=0.3)$ with both temperature and irradiation. Tangential stresses will be considered satisfactory if they do not exceed the tensile strength of graphite.

Substituting $350 \mathrm{MW}$ and the appropriate property values from Figures 2.3-1 and 2.3-2 equation 2.3-1 becomes

$$
D_{p}=0.102\left(V_{b}\right)^{0.5}
$$

where $\mathrm{V}_{\mathrm{b}}$ is bed volume. This relationship is plotted on Figures 2.2-1, 2. 2-2 and 2.2-3 to show the limitations due to thermal stress in the fuel elements.

In order to check the final core design, three locations are of interest: reactor inlet (low temp, low flux), point of maximum flux, and point of maximum temperature. As shown in Section 2.4, a fuel element diameter of $1-1 / 2$ " was selected. This value, along with appropriate values for the properties at these conditions are inserted in equation 2.3-1. The resulting thermal stresses are found to be 465 psi at reactor inlet, 2510 psi at point of maximum flux, and 1830 psi at point of maximum temperature. 


\section{4 Thermal \& Hydraulic Design of Final Core}

Characteristics of the core selected for final design are as follows:

Diameter (equivalent)
Blanket Thickness (equivalent)
Height
Core Voidage
Blanket Voidage
Core Face Area (ball bed)
Blanket Face Area (ball bed)
Total Face Area (ball bed)
Core Volume (ball bed)
Blanket Volume (ball bed)
Core Output
Blanket Output
Total Output

$$
\begin{aligned}
& 9.0 \mathrm{Ft} . \\
& 1.33 \mathrm{Ft} . \\
& 8.1 \mathrm{Ft} \\
& 29 \% \\
& 39 \% \\
& 47.3 \mathrm{Ft} .2 \\
& 44.7 \mathrm{Ft} .2 \\
& 92.0 \mathrm{Ft} .{ }^{3} \\
& 383.0 \mathrm{Ft} .3 \\
& 535.0 \mathrm{Ft} . \\
& 332.5 \mathrm{MW} \\
& 17.5 \mathrm{MW} \\
& 350.0 \mathrm{MW}
\end{aligned}
$$

A shown in Figure 1.4-13 Section 1, the blanket heat generation reaches $8.5 \%$ of the total after about 1000 days of full power operation. However, at startup and continually thereafter, there is about $5 \%$ of the core heat raised in the blanket due to gamma heating. Therefore, at the end of 1000 days about $13.5 \%$ of the heat generated will be in the blanket. Designing to accommodate the heat generated in the blanket is no problem. Maximum heat generated in the core, and the most severe peak to average flux condition, will exist at the time of initial startup with control rods fully inserted. At this time $332.5 \mathrm{MW}$ of heat is generated in the core and 17.5 MW in the blanket, which is used for the design condition.

The core is composed of two sections: a central hexagonal channel and six obround holes in an outer ring. The core is surrounded by the blanket. Flow is in parallel through the core and blanket.

The most severe operating condition, from the standpoint of fuel element temperature is at startup when the control rods are fully inserted. Figure 2,4-1 presents the radial plot of heat generation at this time in both the upper and lower region of the core. Figure 2.4-2 presents an axial plot of heat generation rate. Figure 2.4-3 presents lines of constant heat generation rate based on these data. 


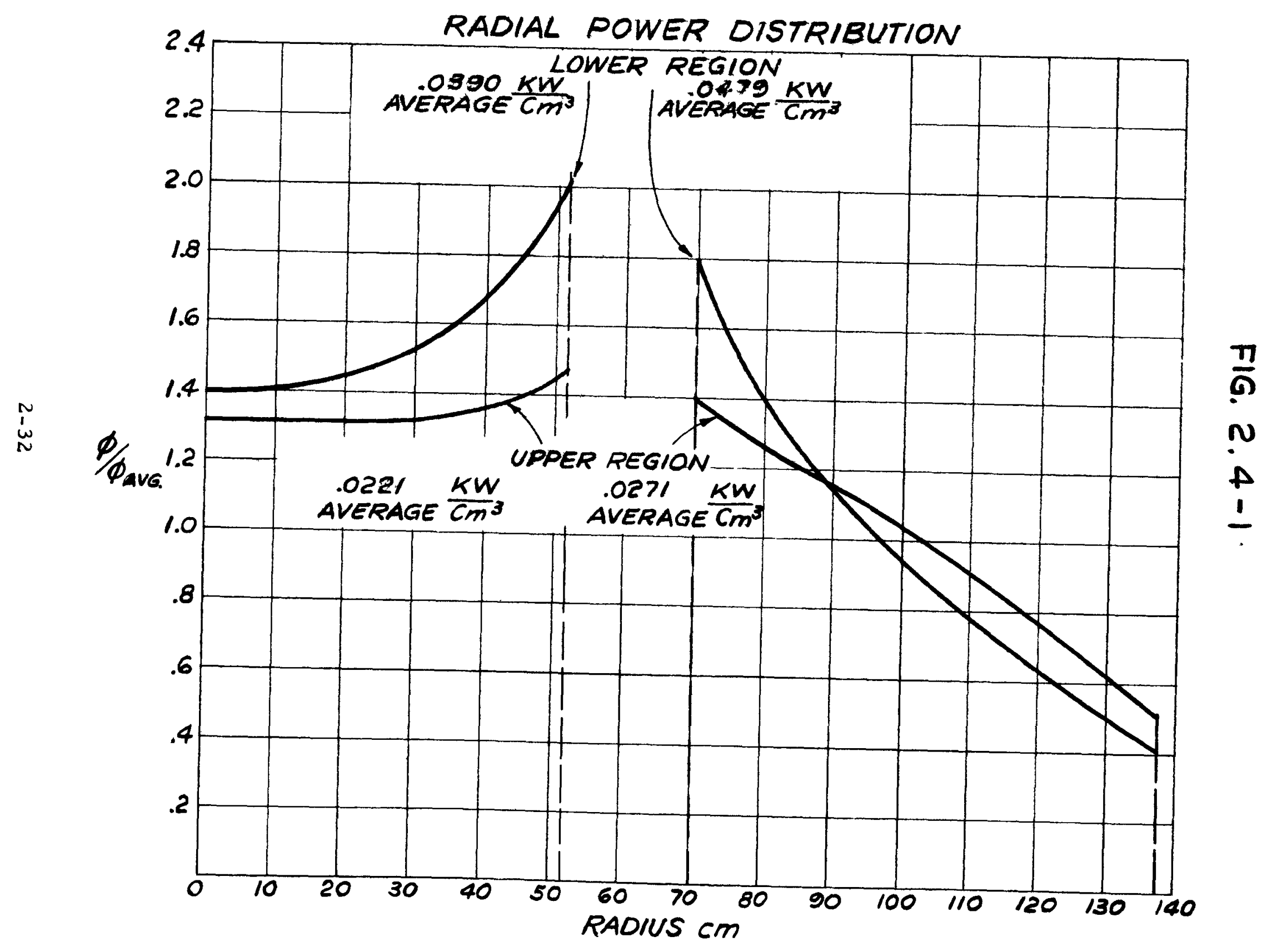

$\pi$
0
1 
FIG. 2, 4-2

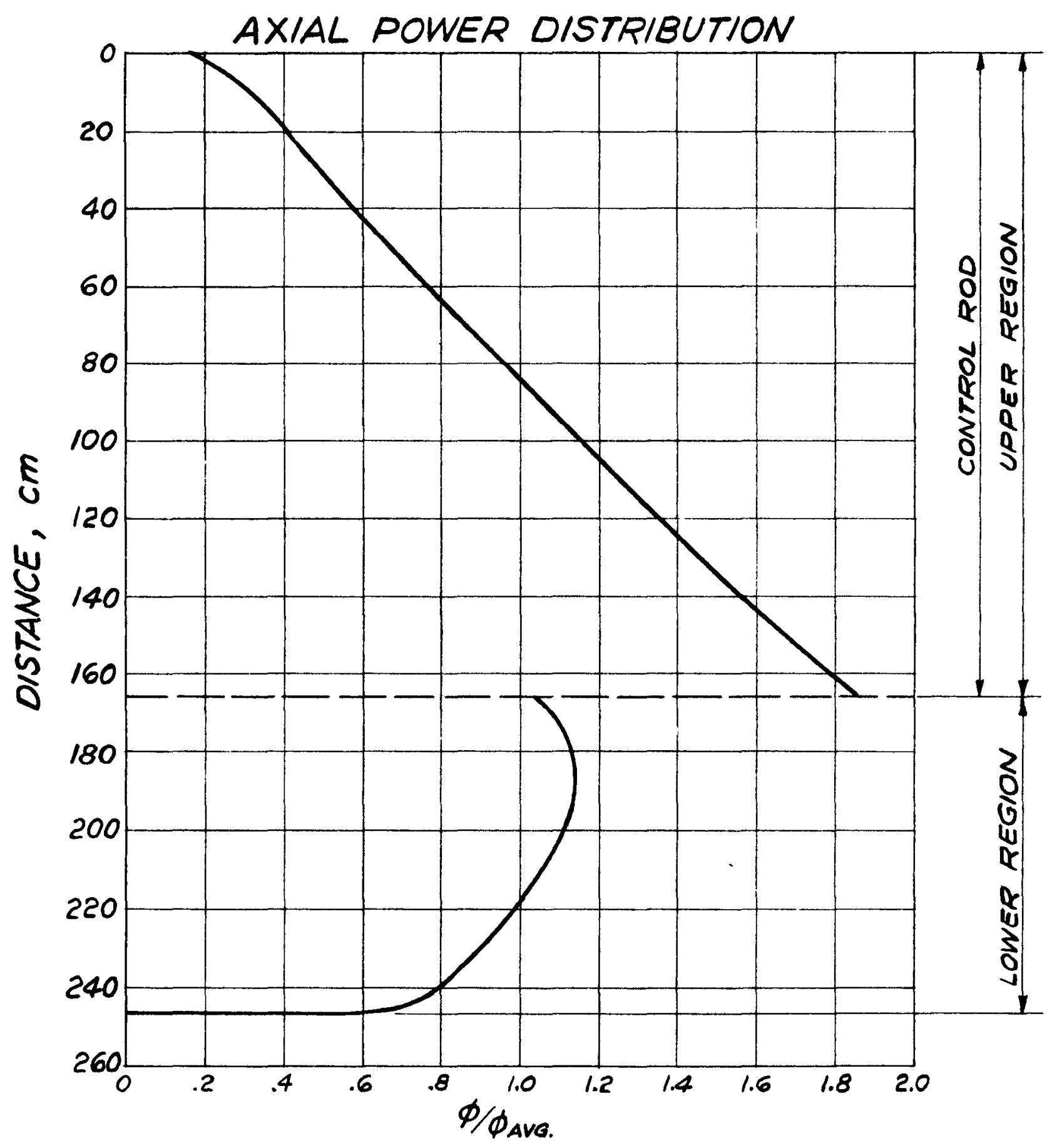

$2-33$ 
FIG. 2 4-3

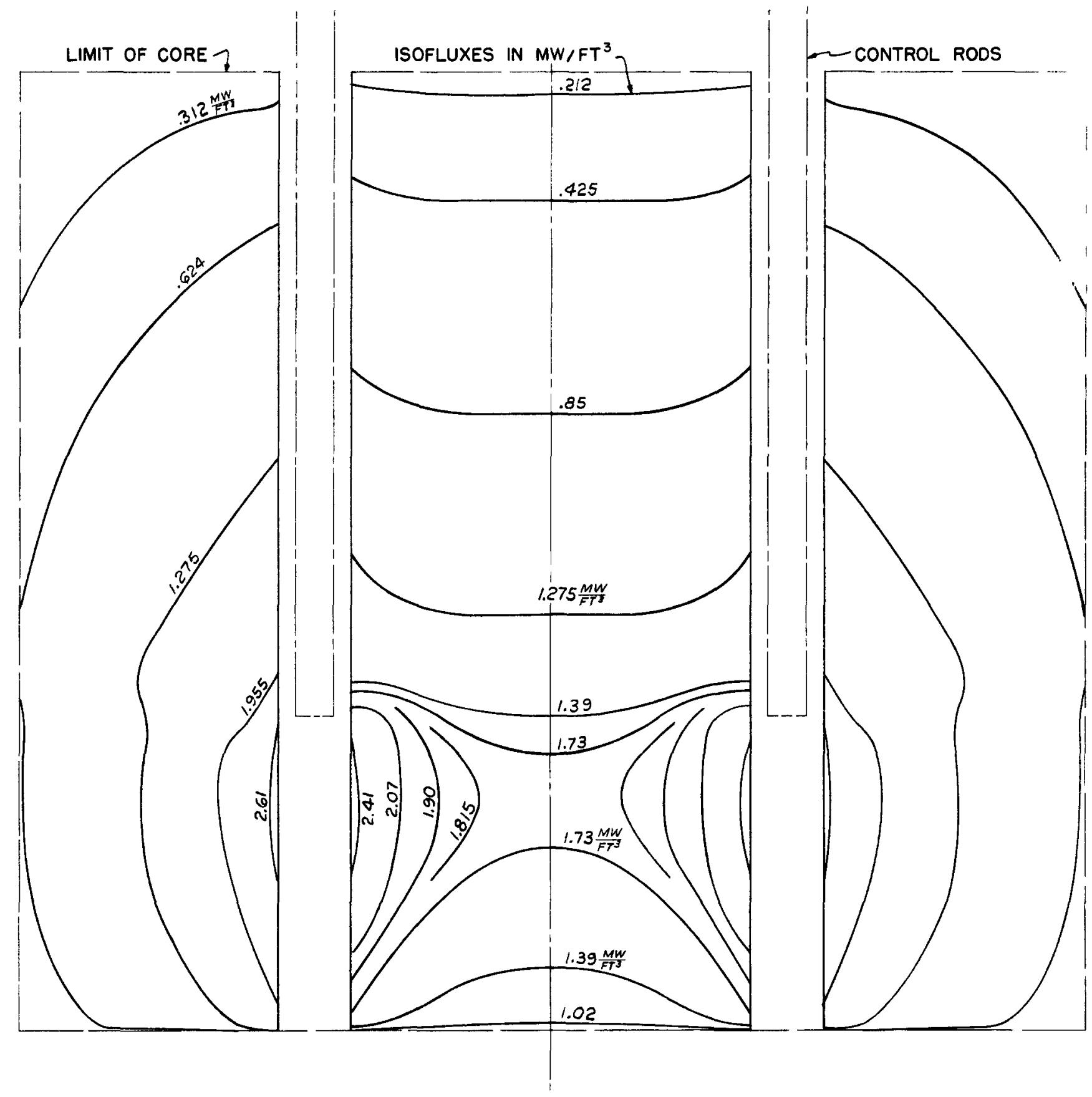

HEAT GENERATION RATES IN $350 \mathrm{MW}$ GORE CONTROL RODS INSERTED 
It should be pointed out that the radial distribution presented in Figure 2.4-1 and replotted in Figure 2.4-3 as sumes a uniform voidage across the bed face. The higher voidage near the wall boundries of the central channel and the rings will reduce the heat generation rates in these regions, as the lower voidage on the axis of these channels will raise the central rate. However, since it is exceedingly questionable if the results of such an analysis would be significant at this time, this refinement was not attempted.

Heat generation in the core decreases radially from the center to essentially zero at the outer wall of the blanket. While allowing the flow to proportion itself between the core and the blanket will result in the lowest pumping power it will result in central fuel element temperatures that are unacceptable as well as extreme radial temperature variations of the helium leaving the core. The other alternative is to divide the flow between the core and blanket in proportion to their heat generation to achieve a more or less uniform temperature pattern of the gas leaving the core.

Flow can be divided between reactor regions by varying ball diameter, orificing, or both. Where it is desired to have the temperature rise between the core and blanket the same, the balls in the core will be about the size of BB's, which is impractical from a design standpoint. On the other hand, if the balls in the core and blanket are the same size the temperature rise through the core will be excessive. Therefore, the area between these two limits was explored to see if an acceptable compromise could be effected without recourse to orificing.

The mean temperature in either the core or blanket is dependent upon the heat generation and the flow through these respective areas.

$$
\delta t_{c}=\frac{\left(\delta t_{r} \times \% \text { heat generated }\right) W_{r}}{W_{C}}
$$

and

$$
\delta t_{b}=\frac{\left(\delta t_{r} \times \% \text { heat generated }\right) W_{r}}{W_{b}}
$$

Substituting design values:

$$
\begin{gathered}
\delta t_{c}=\frac{(700 x .95) 378}{W_{c}}=251,000 / W_{c} \\
\delta t_{b}=\frac{(700 \times .05) 378}{W_{b}}=13,200 / W_{b} \\
2-35
\end{gathered}
$$


The mean temperature is equal to $960+\delta t / 2$ in $R^{\circ}$.

Figure 2.4-4 presents a plot of $\delta$ through the core and blanket vs. $\%$ flow through the core for a power generation of $5 \%$ in the blanket.

Pressure drops through the core and blanket are equal. Pressure drop is a function of weight flow and mean temperature, the mean temperature in turn being dependent upon weight flow.

Eyuation 2.2-2 can be written as:

$$
\Delta P \propto \frac{\mathrm{W}^{1.73} \mathrm{~T}^{1.157} \mathrm{~L}}{\mathrm{P}_{\mathrm{P}}{ }^{1.27} \mathrm{~A}_{\mathrm{s}}^{1.73}}
$$

Rearranging

$$
\mathrm{D}_{\mathrm{p}}^{1.27}=\frac{\mathrm{W}^{1,73} \mathrm{~T}^{1.157} \mathrm{~L}}{\Delta \mathrm{P} \mathrm{PA}_{\mathrm{S}}^{1.73}}
$$

The pressure level in, and pressure drop through, the blanket and core are the same and the ratio of ball diameter between the blanket and core can be expressed as:

$$
\begin{aligned}
& \frac{D_{p_{b}}}{D_{p_{c}}}=\left(\frac{W_{b}}{W_{c}} \frac{A_{c}}{A_{b}}\right)^{1.36}\left(\frac{T_{b}}{T_{c}}\right)^{.91}\left(\frac{L_{b}}{L_{c}}\right)^{79} \\
& A_{c}=47.3 \mathrm{Ft}^{2} \text { and } A_{b}=44.7 \mathrm{Ft}^{2} \\
& \frac{A_{c}}{A_{b}}=1.06 \text { and }\left(\frac{A_{c}}{A_{b}}\right)^{1.36}=1.0825 \\
& \frac{D_{p b}}{D_{p c}}=1.085\left(\frac{W_{b}}{W_{c}}\right)^{1.36}\left(\frac{T_{b}}{T_{a}}\right)^{.91}\left(\frac{L_{b}}{L_{c}}\right)^{.79}
\end{aligned}
$$

The ratio of blanket ball to core ball diameters was solved over a range of values of $W_{b} / W_{c}$ and plotted in Figure 2.4-5 as $D_{p b} / D_{p c}$ and $\delta t_{c}$ vs. $W_{c} / W_{r}$. From the general survey of pressure drop through the core and thermal stress considerations it would appear that the core balls would be $1-1 / 4$ to $1-3 / 4$ inch spheres. From practical and economic considerations the blanket balls should be $3 / 4$ to one inch, indicating that flow through the core should be about $60 \%$ of the total. This will result in an average temperature rise of about $1100^{\circ} \mathrm{F}$ or an outlet 

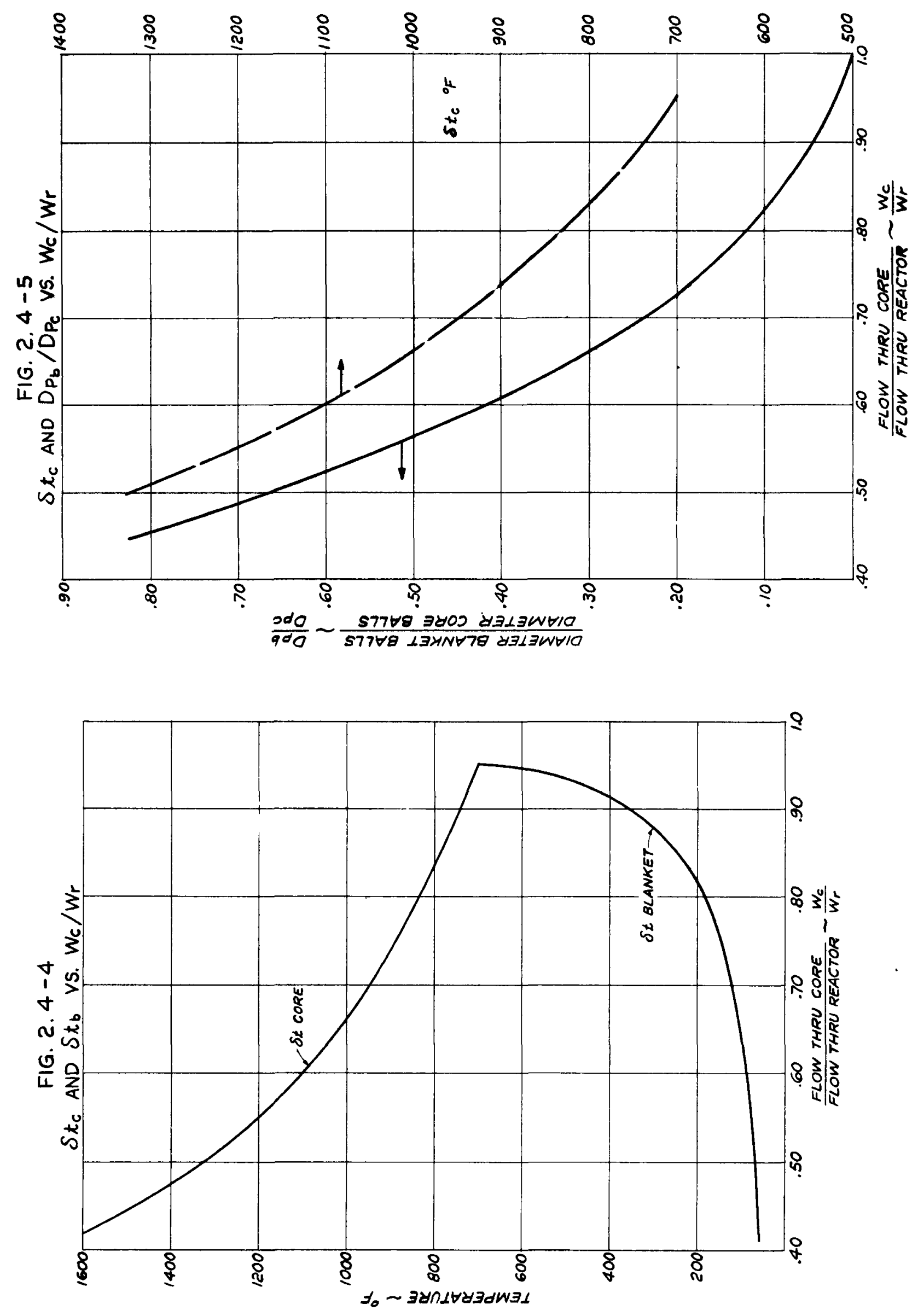
temperature of $1600^{\circ} \mathrm{F}$, which would appear to be an acceptable compromise between core temperature rise, pressure drop and ball diameter.

On the assumption that blanket balls are of the order of $50 \%$ of the diameter of core balls a detailed analysis was carried out including the effect of radial changes in bed voidage. The reactor was divided into seven annular rings, six in the core and one in the blanket. The heat generation in each of these annular elements was determined, ball diameters assigned and the pressure drop determined for variations in weight flow. Characteristics of the annular rings are given in Table 2.4-1. A plot of $W$ vs. $\beta$, where $W$ is the channel flow in $1 \mathrm{bs} / \mathrm{sec}$ and $\beta$ the ratio of pumping power to heat transferred for the reactor as a whole, is given in Figures 2.4-6, 2.4-7 and 2.4-8 for three combinations of ball diameters. The design point is determined graphically from the sum of the weight flows through the individual sections. Figure 2. 4-9 presents the mean temperature rise through the core for these three selected conditions. It will be seen that case $B$, the combination of 1-1/2 inch balls in the core and $3 / 4$ inch balls in the blanket, results in the most uniform temperature profile and the lowest $\Delta \mathrm{T}$ between regions, which reduces the thermal stress in the fuel cylinders. The combination of 1-1/2 inch core balls and 3/4 inch blanket balls was selected on this basis and used for final design. Table 2.4-2 presents final design conditions for the core. Figure 2.4-10 presents the temperature rise through the core, outlet temperature and ball surface temperature in regions 2 and 4 , the most highly loaded areas in the core.

At the end of 1000 days the blanket heat generation is $8.5 \%$ plus $5 \%$ for gamma heating or $13.5 \%$ of reactor output. At this time the core is generating $86.5 \%$ of the power, resulting in lower core ball temperature than at the time of startup. 
FIG. $2.4-6$

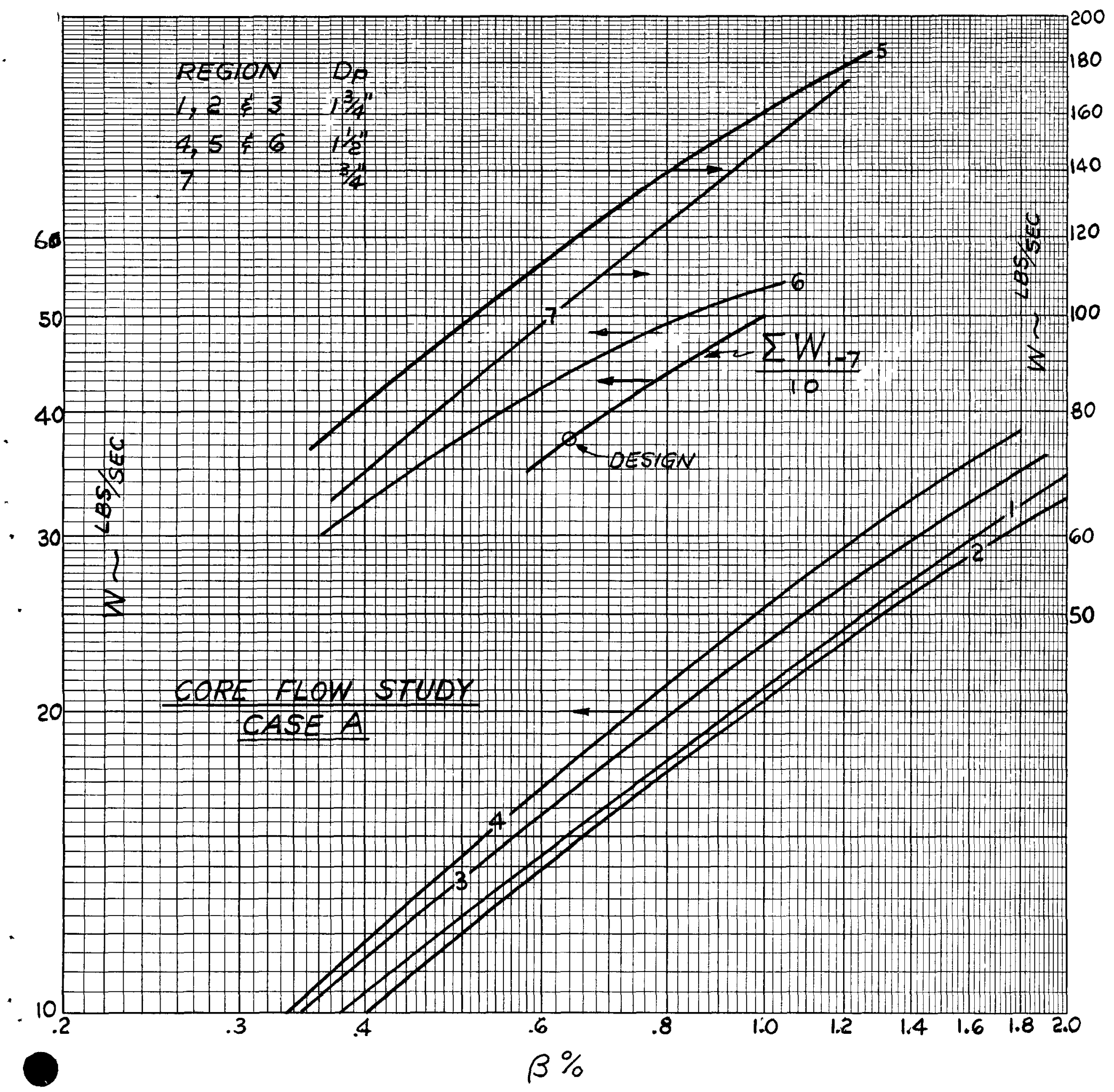


FIG. 2. 4-7

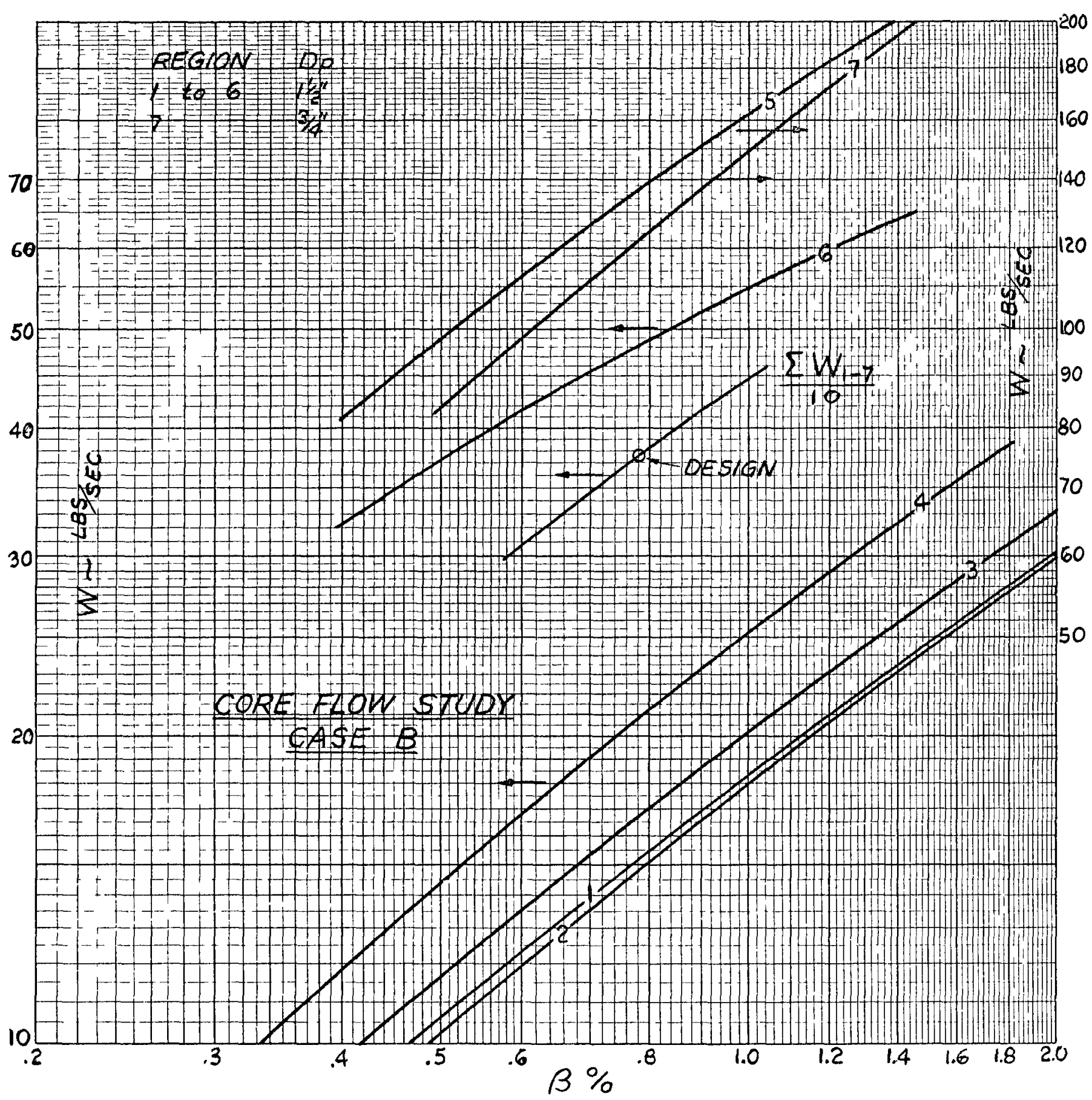


FIG. 2. 4-8

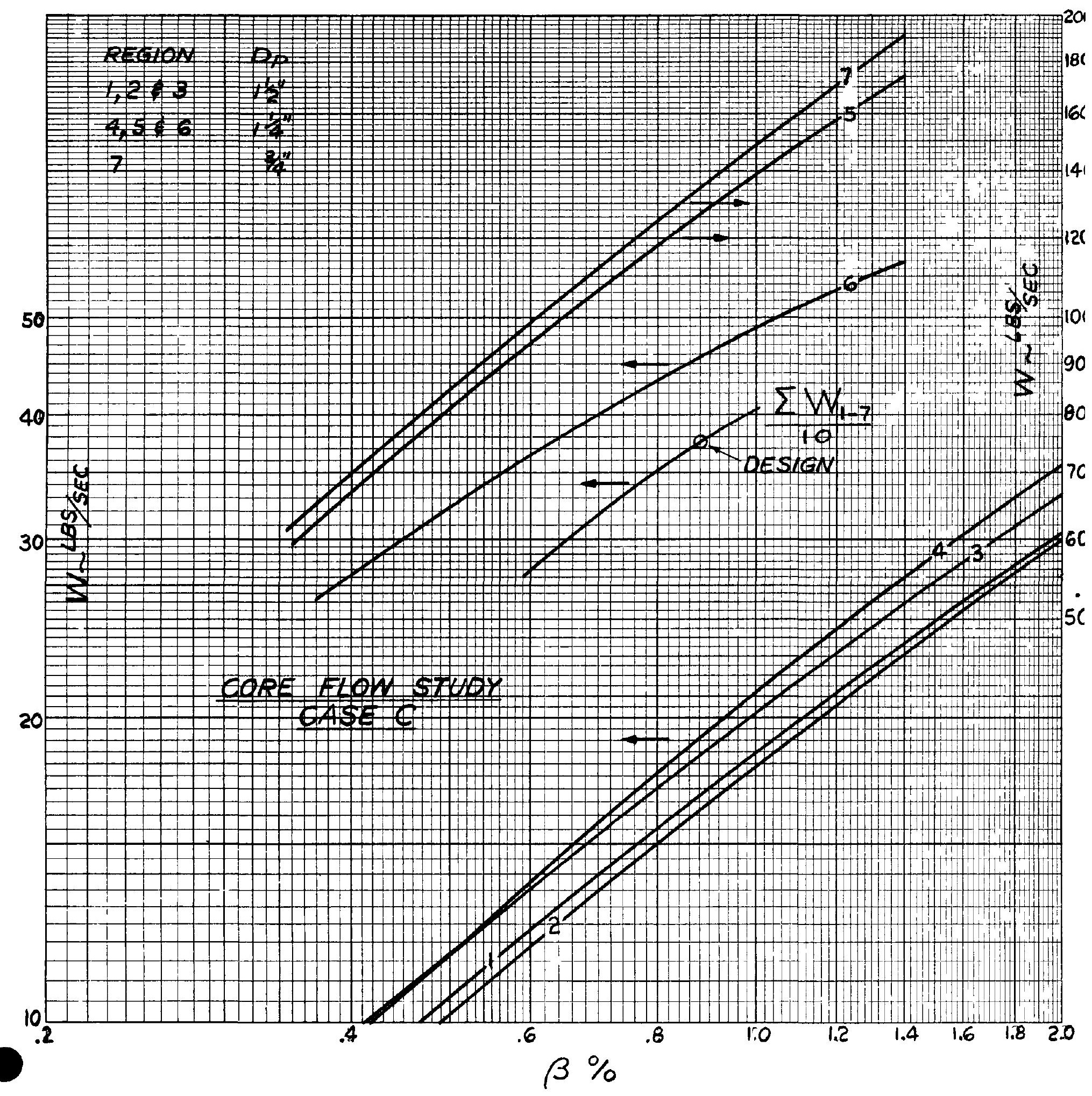


FIG. 2. 4-9

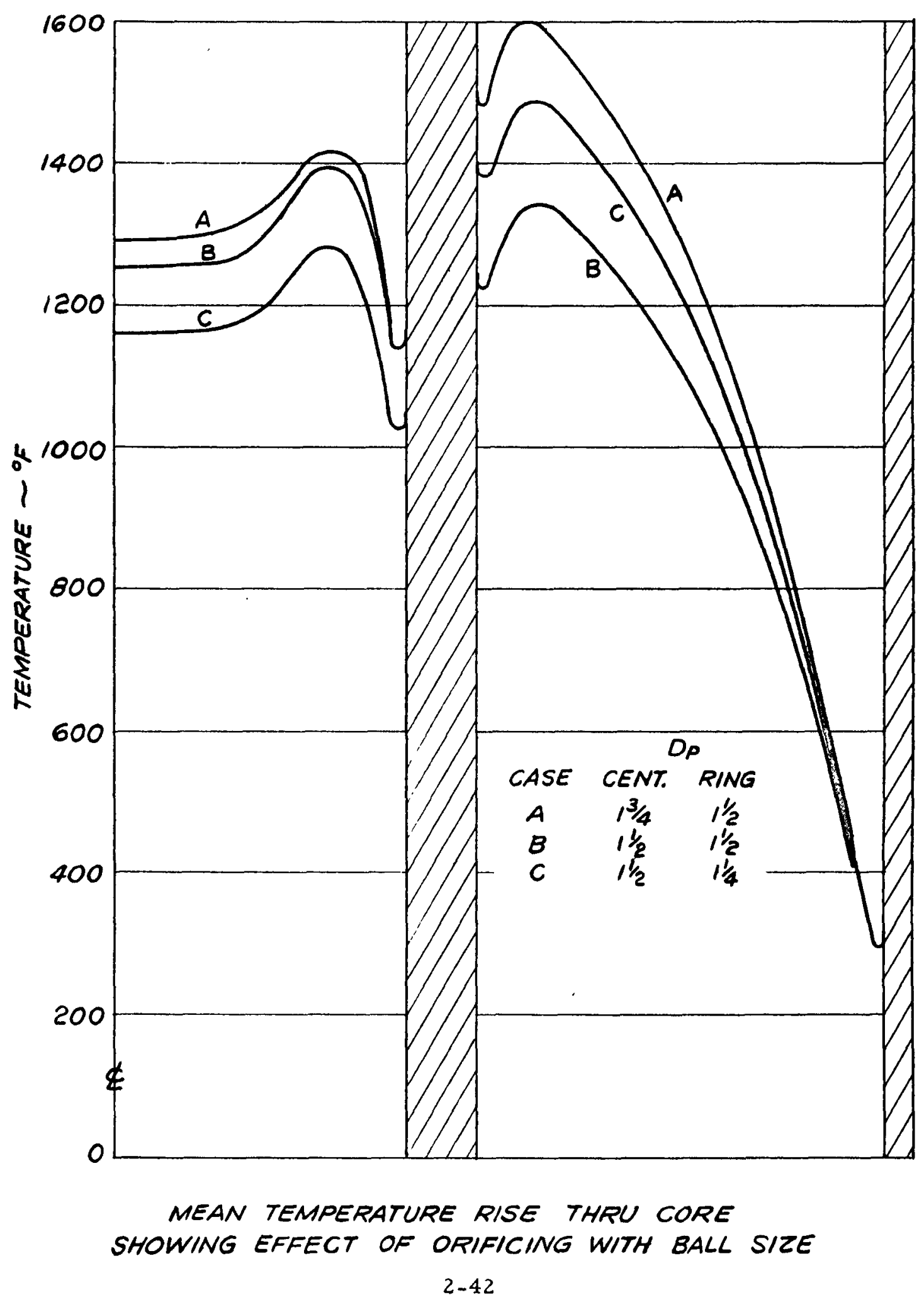


FIG. 2. 4-10

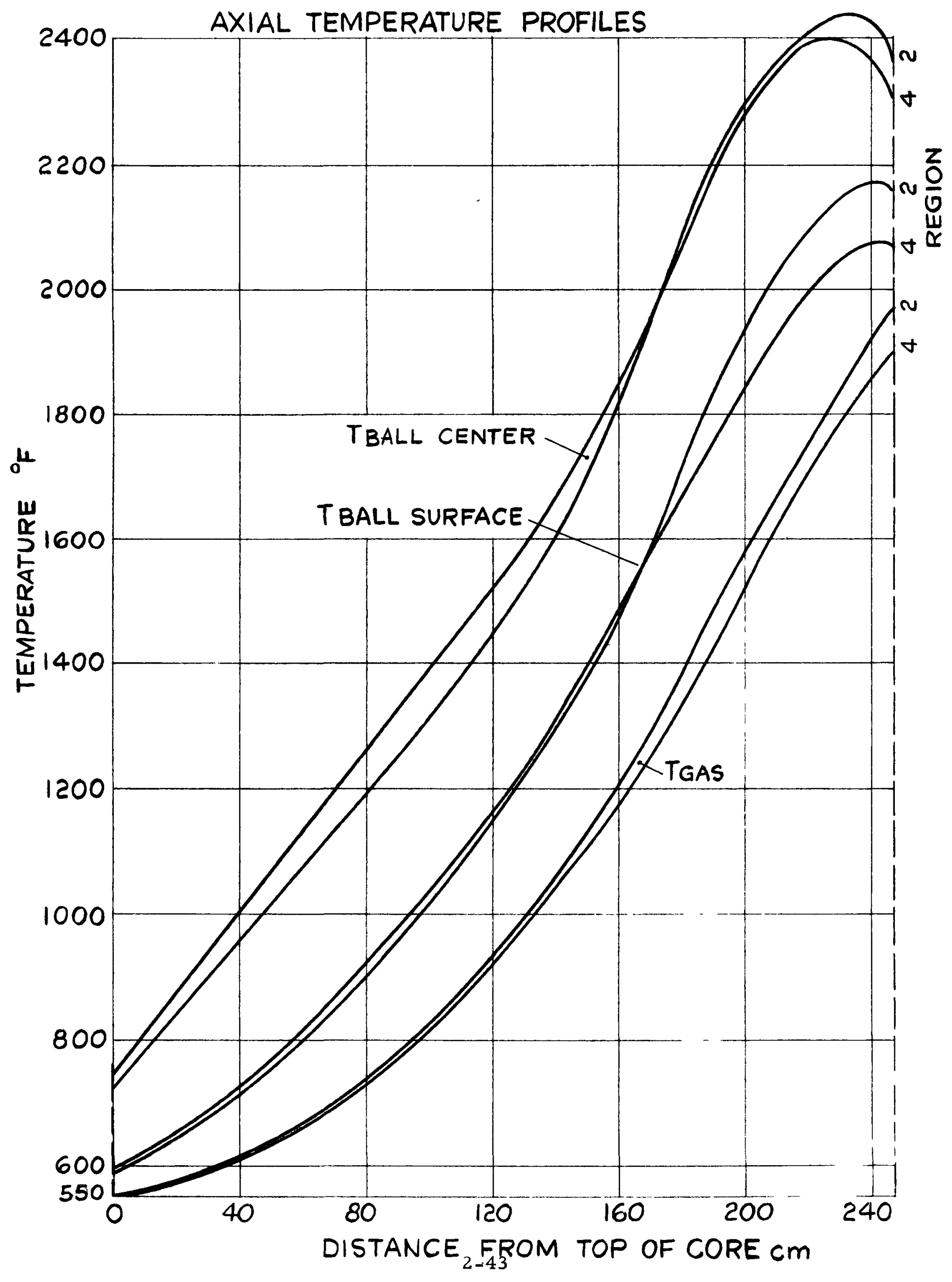


TABLE - 2.4-1

Characteristics of Annular Rings Used in Final Core Analysis

\begin{tabular}{|c|c|c|c|c|c|c|c|c|}
\hline Region & 1 & 2 & 3 & 4 & 5 & 6 & 7 & Total \\
\hline$A_{s}-F t^{2}$ & 3.1 & 3.1 & 2.8 & 3.6 & 27.8 & 6.9 & 44.7 & 92.0 \\
\hline$r *-$ In. & 8.42 & 14.64 & 18.73 & 29.33 & 41.72 & 52.1 & - & - \\
\hline $\mathrm{L}-\mathrm{Ft}$ & 8.1 & 8.1 & 8.1 & 8.1 & 8.1 & 8.1 & $11.1 * *$ & - \\
\hline$V-F t^{3}$ & 25.1 & 25.1 & 22.7 & 29.2 & 225.0 & 55.9 & 362 & 745 \\
\hline$Q-M W$ & 25.45 & 27.45 & 27.40 & 37.56 & 188.35 & 26.29 & 17.5 & 350 \\
\hline$\% Q$ & 7.28 & 7.85 & 7.83 & 10.73 & 53.80 & 7.51 & 5.0 & 100 \\
\hline$q-M W / F t^{3}$ & 1.012 & 1.095 & 1.205 & 1.285 & 0.84 & 0.47 & 0.048 & - \\
\hline$W \delta t / 1000$ & 19.27 & 20.8 & 20.7 & 28.4 & 142.3 & 19.9 & 13.23 & \\
\hline$\varepsilon \%$ & 37 & 37 & 41.5 & 41.5 & 37 & 41.5 & 39 & \\
\hline $\mathrm{f} R \mathrm{e}^{.27}$ & 74.3 & 74.3 & 53.5 & 53.5 & 74.3 & 53.5 & 67.5 & \\
\hline
\end{tabular}

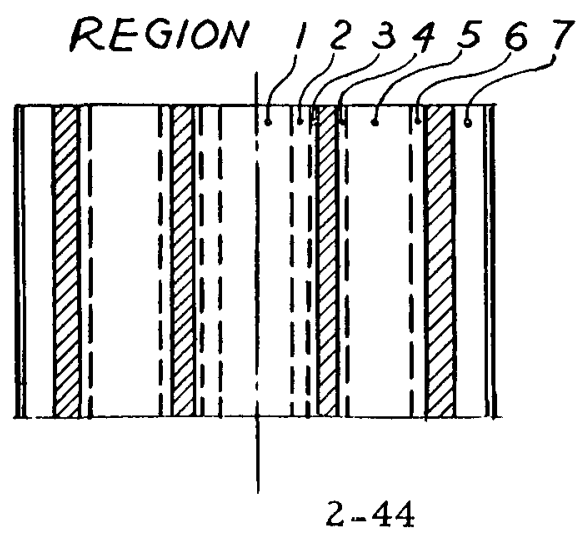


TABLE $-2.4-2$

Summary of Hydraulic \& Thermal Performance in Core Regions

\begin{tabular}{|c|c|c|c|c|c|c|c|c|}
\hline Region & 1 & 2 & 3 & 4 & 5 & 6 & 7 & Total \\
\hline$A_{s}-F^{2}$ & 3.1 & 3.1 & 2.8 & 3.6 & 27.8 & 6.9 & 44.7 & 92.0 \\
\hline$V-F t^{3}$ & 25.1 & 25.1 & 22.7 & 29.2 & 225.0 & 55.9 & 362 & 745 \\
\hline$D_{p}=1 n$ & 1.5 & 1.5 & 1.5 & 1.5 & 1.5 & 1.5 & 0.75 & - \\
\hline$S=F t^{2}$ & 735 & 735 & 665 & 855 & 6600 & 1640 & 22200 & 33430 \\
\hline$Q \cdot M W$ & 25.45 & 27.45 & 27.40 & 37.56 & 188.35 & 26.29 & 17.5 & 350 \\
\hline$R=\frac{B T U}{H r \cdot F t^{2} \cdot 10^{3}}$ & 118.0 & 127.5 & 140.5 & 150.0 & 97.4 & 54.8 & 2.62 & \\
\hline $\mathrm{W}-\mathrm{Lbs} / \mathrm{sec}$ & 15.3 & 14.9 & 16.9 & 21.2 & 138.2 & 48.5 & 123.0 & 378 \\
\hline$G-L b s / s e c / F t^{2}$ & 4.94 & 4.81 & 6.04 & 5.88 & 4.98 & 7.04 & 2.75 & \\
\hline $\mathrm{T}_{1 \mathrm{n}}-{ }^{2} \mathrm{~F}$ & 550 & 550 & 550 & 550 & 550 & 550 & 550 & \\
\hline$\delta_{\mathrm{t}}={ }^{\circ} \mathrm{F}$ & 1260 & 1395 & 1225 & 1340 & 1030 & 410 & 108 & \\
\hline $\mathrm{T}_{\text {out }}-{ }^{\curvearrowright} \mathrm{F}$ & 1810 & 1945 & 1775 & 1890 & 1580 & 960 & 658 & \\
\hline$\Delta \mathrm{T}_{\mathrm{f}^{-}} \mathrm{F}$ & 195 & 213 & 202 & 218 & 162 & 74 & 6 & \\
\hline $\mathrm{T}_{\mathrm{S}} \max { }^{\circ} \mathrm{F}$ & & 2170 & & 2075 & & & & \\
\hline$\rho \operatorname{avg} 1 b / F^{3}$ & .224 & .215 & .2263 & .2187 & .241 & .3022 & .345 & \\
\hline$v_{s}$ avg Ft/sec & 22.0 & 22.4 & 26.7 & 26.9 & 20.7 & 23.3 & 8.0 & \\
\hline
\end{tabular}




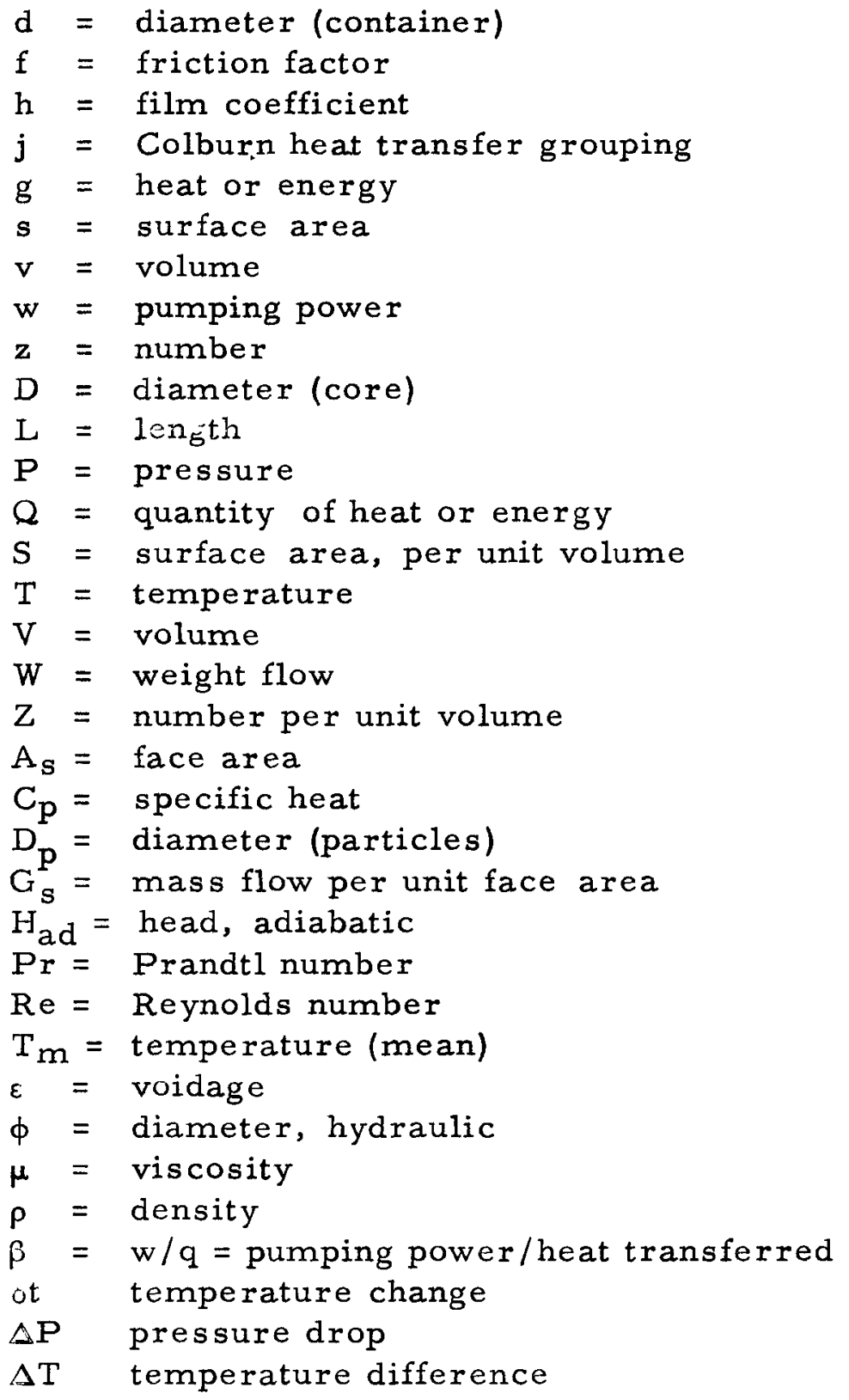

Subscripts

$$
\begin{aligned}
& \mathrm{p}=\text { pump } \\
& \mathrm{c}=\text { core } \\
& \mathrm{b}=\text { blanket } \\
& \mathrm{r}=\text { reactor }
\end{aligned}
$$


(1) Denton, W. H., "The Packing and Flow of Spheres", AERE E/R 1095; January 1953.

(2) Carman, P. C., "Fluid Flow Through Granular Beds", Trans actions of the Institution of Chemical Engineers, Vol. 15, 1937, pg. 150 .

(3) Schwartz, C. E. and J. M. Smith, "Flow Distribution in Packed Beds," Industrial and Engineering Chemistry Vol. 45, No. 6; June 1953.

(4) Shortell, J. W., "Experimental Determination of Some Characteristics of Packed Sphere Beds", D-T-21, California Research and Development Co; August, 1953.

(5) Kays, W. M. and A. L. London, Compact Heat Exchangers; The National Press, 1955.

(6) Denton, W. H., C. H. Robinson, and R. S. Tibbs, "Heat Transfer and Pressure Loss in Fluid Flow Through Randomly Packed Spheres"; HPC-35, June, 1949.

(7) Glasser, M. B., and G. Thodos, "Heat \& Momentum Transfer in the Flow of Gases Through Packed Beds" Ph..D.Thesis North Western Technical Institute, Evanston, Ill.

(8) Brownell, L. E., H. S. Dombrowski, and C. A. Dickey, "Pressure Drop through Porous Media" C.E.P. 46 415-422 (1950).

(9) Rose, H. E., and A.M.A. Rizk, "Further Researches in Fluid Flow through Beds of Granular Material", Paper to the Institute of Mechanical Engineers, Dec. 10, 1948.

(10) Graton, L. C. and H. J. Fraser, "Systematic Packing of Spheres With Particular Relation to Porosity and Permeability",

Journal of Geology, Vol. XLIII, No. 8, Part I, Nov.-Dec. 1935.

(11) Coulson, "The Streamline Flow of Liquids through Beds Composed of Spherical Particles," Univ. of London Ph. D. Thesis, 1935.

(12) Denton, W. H., "The Heat Transfer and Flow Resistance for Fluid Flow Through Randomly Packed Spheres," IME and ASME Proceedings of the General Discussion on Heat Transfer, May 1951.

(13) Gamson, B, G. Thodos, and O. Hougen, "Heat, Mass and Momentum Transfer in the Flow of Gases thru Granular Solids" Trans. AICHE 39 (1943)

(14) Coppage, J. E., "Heat Transfer and Flow Characteristics of Porous Media," T.R. No. 16 (N6 ONR-251), December 1952. 
(15) Daniels, "Suggestions for a High Temperature Pebble Pile", $\mathrm{N}-1668 \mathrm{~b}$, October 1944.

(16) Roman et al. , "Preliminzry Report on the Application of Fluidized \& Fixed Pellet Beds to Power Reactors", WAPD-Re-(AE)-11.

(17) Beely, A Pebble Bed Reactor for Stationary Power Plants", NAA-SR-895, February, 1954.

(18) NDA Staff, "Design of an Experimental Reactor for High Operating Temperatures". NDA-64-101, September 1954.

(19) ORSORT, "Gas-Cooled Pebble Bed Reactor for a Large Central Station", CF-57-8-12, August 1957.

(20) S\&P Memo, "Visit of Claus B. Van der Decken of F. Krupp, AG of March 1958.

(21) "Properties of Materials" The Reactor Handbook, Vol. 3, Sect. 1; AECD-3647, 1955.

(22) Currie, L. M., V. C. Hamister \& H. G. MacPherson, "The Production \& Properties of Graphite" National Carbon Company, 1955.

(23) Loch, L. D., J.A. Slyth \& W.H. Duckworth, "Studies of Graphite for Fuel Elements", TID 10001, October 13, 1954.

(24) Durand, R.E., D.J. Klein \& F.R. Nykiel, "Effect of Reactor Irradiation on the Thermal Conductivity of Uranium Impregnated Graphite at Elevated Temperature", NAA-SR-836(De1.), April 29, 1954.

(25) Hunter, L.P., "Progress on High Temperature Thermal Conductivity Measurements", Mon-N-442, December 16, 1947. 
Section 3.0

Fuel Cycle Economics

3.0.1 Fuel element fabrication costs $\ldots \ldots \ldots \ldots \ldots \ldots \ldots \ldots$ 3-1

3.0.2 Fertile material cost ..................... 3-3

3.0.3 Fissile material cost ....................

3.0.4 Fuel reprocessing costs $\ldots \ldots \ldots \ldots \ldots \ldots \ldots \ldots \ldots \ldots 3-3$

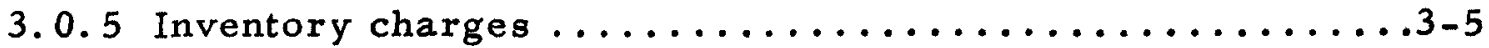

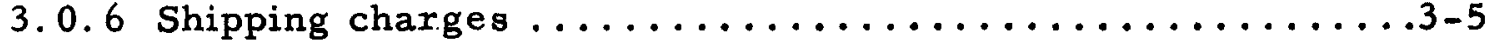

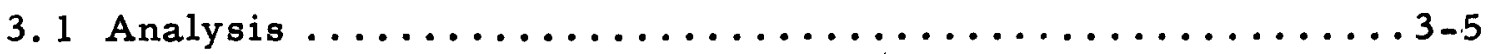

3.2 Results of Analysis $\ldots \ldots \ldots \ldots \ldots \ldots \ldots \ldots \ldots \ldots \ldots \ldots, 13$

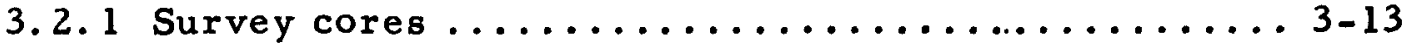

3.2.2 Design core ........................ 3-16

3.2.2.1 Fuel burnup and losses ............. 3-16

3.2.2.2 Inventory ................... 3-17

3.2.2.3 Reprocessing ...................... 18

3.2.2.4 Core and blanket element fabrication ......3-18

3.2.2.5 Shipping costs ................... 3-19

3.2.2.6 Fuel cycle costs .................. 3-19

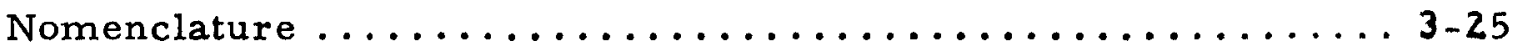




\subsection{Fuel Cycle Economics}

A nuclear, thermal and fluid dynamic survey has been made of a series of cores, ranging from 7 to 20 feet in diameter, that might be suitable for the $125 \mathrm{eMW}-\mathrm{PBR}$. In order to provide guidance for the selection of a final core, the fuel cycle economics of this series of cores has been studied. A consideration of the following items formed the basis of this study:

\subsubsection{Fuel Element Fabrication Costs}

Manufacturing costs for core and blanket elements have been obtained from a number of vendors. As would be expected there is a wide variation in prices, depending upon whether the source is an industrial firm or a research laboratory and whether or not the vendor had previously handled fissile and fertile material.

Molded spheres of reactor grade graphite of density 1.70 grams/ cc have been quoted in quantities of 100,000 as follows:

\begin{tabular}{lcr} 
Size & Unit Price, $\notin$ & \$/Lb \\
\cline { 2 - 3 } $1{ }^{\prime \prime}$ & 4.7 & 1.46 \\
$1-1 / 4^{\prime \prime}$ & 6.7 & 1.07 \\
$1-1 / 2^{\prime \prime}$ & 8.8 & .82 \\
$2 " 1$ & 13.9 & .54
\end{tabular}

Plus a $\$ 700$ charge for die.

These prices are plotted in Figure 3.0-1. An element of this type is not used in this reactor and its price is noted for information only.

Core elements are composed of 53.88 grams of graphite, 4.94 grams of $\mathrm{ThO}_{2}$ and 0.448 grams of $\mathrm{UO}_{2}$. Estimates have been received on the manufacture of such elements by two different methods, impregnation, and admixture. Prices on finished elements $1-1 / 2$ inches in diameter less fissile material, as manufactured by these methods are as follows:

Impregnation

Admixture
$37 \phi$

$56 d$ low, $76 d$ high 
FIGURE 3.0-1

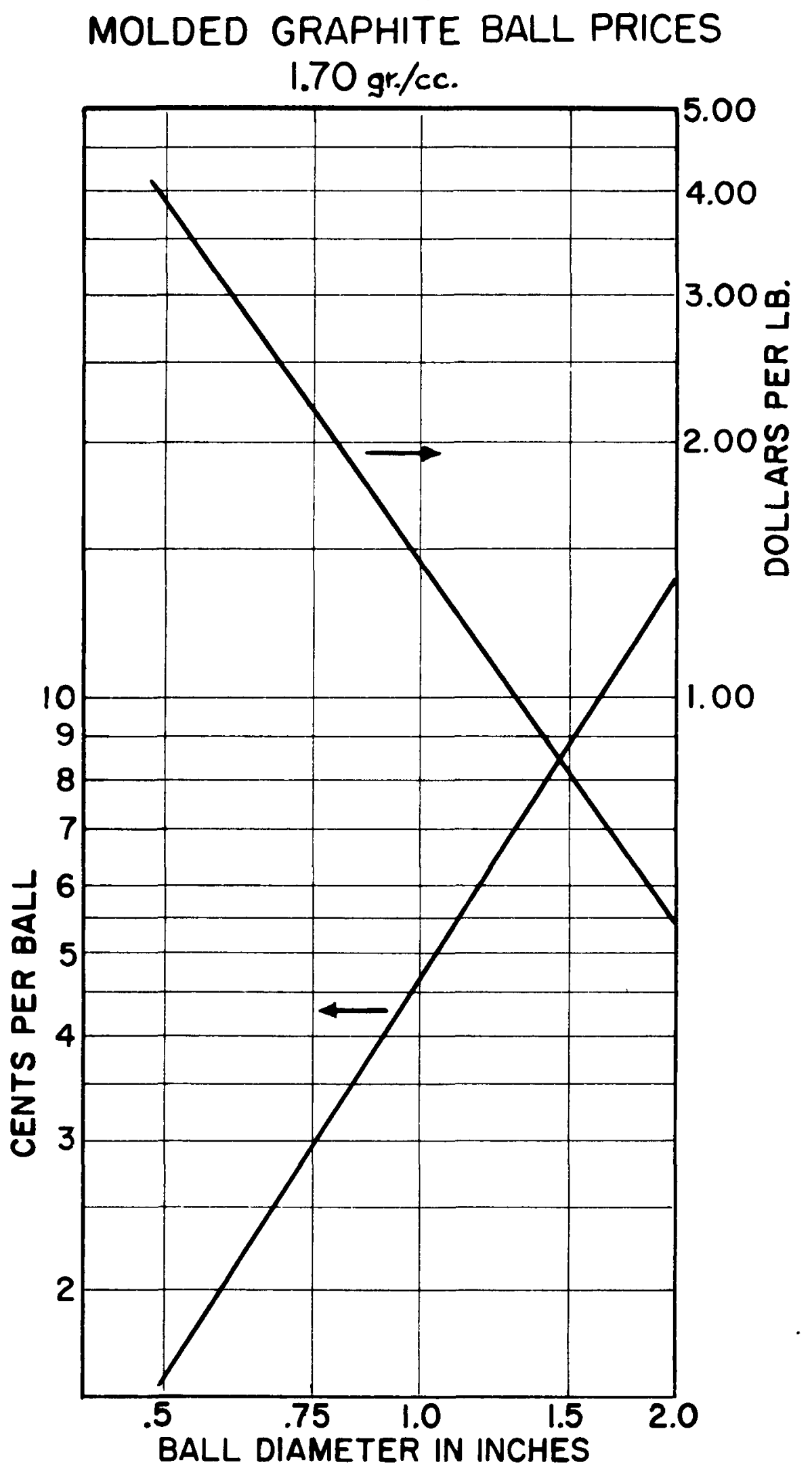


Blanket elements are composed of 5.66 grams of graphite and 5.66 grams of $\mathrm{ThO}_{2}$ Estimates have been received on the manutacture of such elements by two different methods; admixture and lumping. Prices on finished elements as manufactured by these methods are presented in Figure 3.0-2. A $3 / 4$ inch element has been selected for this design which has a low price of $16.5 \not$ and a high price of $55 \not$, both including the cost of the fertile material.

\subsubsection{Fertile Material Cost}

Thorium cost, as $\mathrm{ThO}_{2}$, was taken as $\$ 17.00$ per kilogram of Th-232. This corresponds to a $\mathrm{ThO}_{2}$ price of $\$ 6.78$ per pound.

\subsubsection{Fissile Material Cost}

Fully enriched U-233 was taken at $\$ 15,000$ per kilogram.

\subsubsection{Fuel Reprocessing Costs}

Fuel reprocessing costs a re based on quotations given by the AEC for a conceptual multipurpose plant capable of handling any type of fuel element.(WASH-743). Quotations given do not include a separate item for head end processing and while we are of the opinion that the head end reprocessing costs for a graphite matrix element will be appreciably less than for a "canned" element, there is no way of objectively adjusting these costs. We have therefore used the basic quote of $\$ 15,300$ per day, realizing that it is conservative.

Plant throughput is based on one of three factors:

1) A maximum of $40 \mathrm{Kg}$ per day of fully enriched U-233.

2) A maximum of $600 \mathrm{Kg}$ per day of $\mathrm{Th}-232$, if the $\mathrm{Th}-232$ is to be saved.

3) A maximum of $1000 \mathrm{Kg}$ per day of $\mathrm{Th}-232$ is to be discarded.

Reprocessing plant usage charge was taken as $\$ 15,300$ per day including turn around time, covering plant cleanup and maintenance. Turn around time was determined according to the following limits:

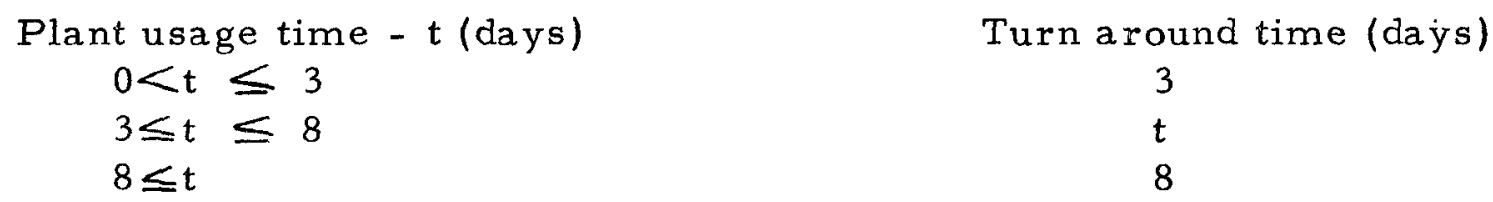


FIGURE 3.0-2

BLANKET BALL COST ESTIMATE $50 \%$ GRAPHITE $50 \% \mathrm{ThO}_{2}$

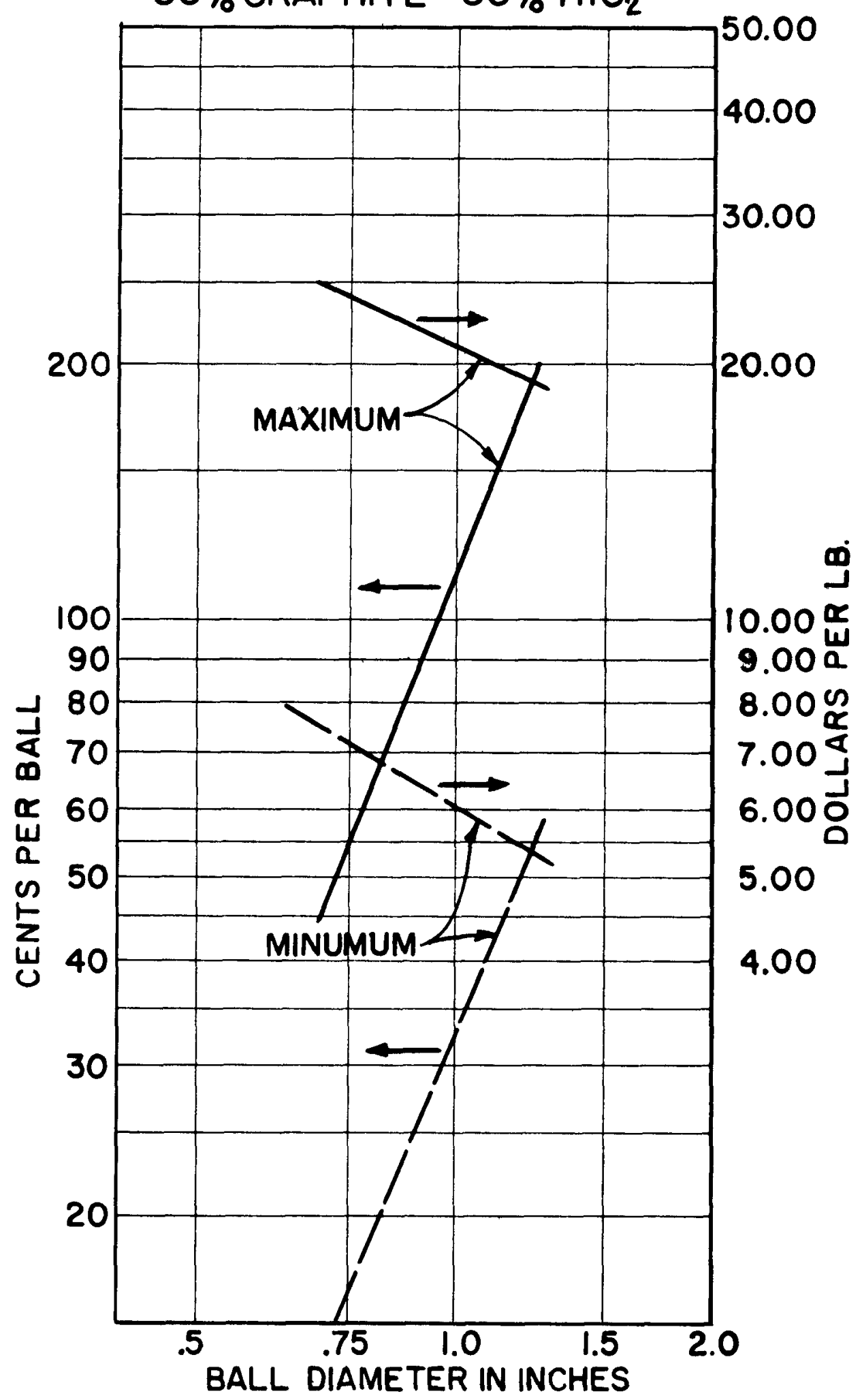




\subsubsection{Inventory Charges}

It is assumed that all material other than U-233 is purchased. $\mathrm{U}-233$ is leased from the AEC at $4 \%$ per year of its value at $\$ 15.00$ per gram over the following periods:

1) Fuel element fabrication

2) Spare core residence period

3) Reactor (core \& blanket)residence time

4) Cooling time

5) Shipping and reprocessing time

\subsubsection{Shipping Charges}

Utilizing shipping costs published for the 500 tMW-PWR at Shipping port and those of a hypothetical $500 \mathrm{tMW}-\mathrm{PWR}$ as reported in Nucleonics (Jan. 1955), together with the assumptions that the total shipping weight is proportional to the burn-up level and that the distance from the reactor site to reprocessing facility is 2000 miles, a spent core shipping cost has been estimated at $\$ 37,000$. This includes shipping cask write-off, cooling requirements, guards, insurance, etc. This is the equivalent of $1.3 \notin / 10^{6} \mathrm{BTU}$, based on a core output of $2.87 \times 10^{12} \mathrm{BTU}$. Since the distance of 2000 miles is estimated as well as the weight ratio of shipping cask to spent fuel, a low charge of $1.0 \not / 10^{6} \mathrm{BTU}$ and a high charge of $1.5 \notin / 10^{6} \mathrm{BTU}$ was used in the estimates. This falls within the limits as reported in the above referenced study in Nucleonics.

\subsection{Analysis}

Fuel cycle cost calculations are based in the assumptions set forth in Section 3.0, plus the following:

a) After extraction from the reactor, blanket and core material are cooled at the reactor site.

b) After extraction from the reactor, blanket and core material are combined to yield one batch of material for reprocessing.

c) Schedule is arranged so that a portion of the blanket and core cooling time is used in transit time to the reprocessing facility.

d) Reprocessing losses amount to $1 \%$ of uranium and thorium reprocessed.

e) Thorium quantities dictate that reprocessing economics be based on thorium throughput. 
Reprocessing costs as a function of thorium batch size are presented in Figure 3.1-1 for both 600 and $1000 \mathrm{Kg}$ per day throughput rate. Below batch sizes of $5000 \mathrm{Kg}$, there is no significant difference in cost between the alternates of saving or discarding the thorium. Above $5000 \mathrm{Kg}$, it is obviously more economical to recover the thorium.

Fuel element (core and blanket) cooling time has been examined to determine the effect of the 27.4 day half life of $\mathrm{Pa}-233$ on fuel cycle economics. The thorium cycle breeding reactions are as follows:

$$
T_{h}^{232} n, \gamma>\operatorname{Th}^{233} \frac{\beta^{-}}{23.3 m} P^{233} \frac{\beta^{-}}{27.4 d} U^{233}
$$

The buildup equations for the breeding reaction are:

$\frac{d N^{03}}{d t}=\left[\sigma_{a t h}^{02} \phi_{t h}+\sigma_{a f}^{02} \phi_{f}\right] N^{02}-N^{03}\left[\lambda^{03}+\sigma_{a t h}^{03} \phi_{t h}+\sigma_{a f}^{03} \phi_{f}\right]$

$$
N^{03}=\frac{\left[\sigma_{a t h}^{02} \phi_{t h}+\sigma_{a f}^{02} \phi_{f}\right] N^{02}}{\lambda^{03}+\sigma_{a t h}^{03} \phi_{t h}+\sigma_{a f}^{03} \phi_{f}}\left[1-e^{-\left(\lambda^{03}+\sigma_{a t h}^{03} \phi_{t h}+\sigma_{a t h}^{03} \phi_{f}\right) t}\right] \quad 3.1-3
$$

Since ${ }^{\mathrm{t}}{ }_{1 / 2}{ }^{03}=23.3 \mathrm{~m}, \lambda 03 \gg \sigma_{\mathrm{ath}}^{03} \emptyset_{\mathrm{th}}+\sigma_{\mathrm{af}} 03 \emptyset_{\mathrm{f}}$ so that at any time after a day's operation $\mathrm{N}^{03}$ is constant.

$$
\frac{d N^{\prime 3}}{d t}=N^{03} \lambda^{03}-N^{13}\left[\lambda^{13}+\sigma_{a t h}^{13} \phi_{t h}+\sigma_{a f}^{13} \phi_{f}\right]
$$

assuming $\lambda^{13}>\sigma_{a t h}^{13} \phi_{t h}+\sigma_{a f}^{13} \phi_{f}$

$$
N^{13}=\frac{N^{03} \lambda^{03}}{\lambda^{13}}\left(1-e^{-\lambda^{\prime 3} t}\right)=\frac{C}{\lambda^{13}}\left(1-e^{-\lambda^{\prime 3} t}\right)
$$


FIGURE $3.1-1$

THORIUM REPROCESSING COST BASED ON USE OF AEC MULTI-PURPOSE PLANT

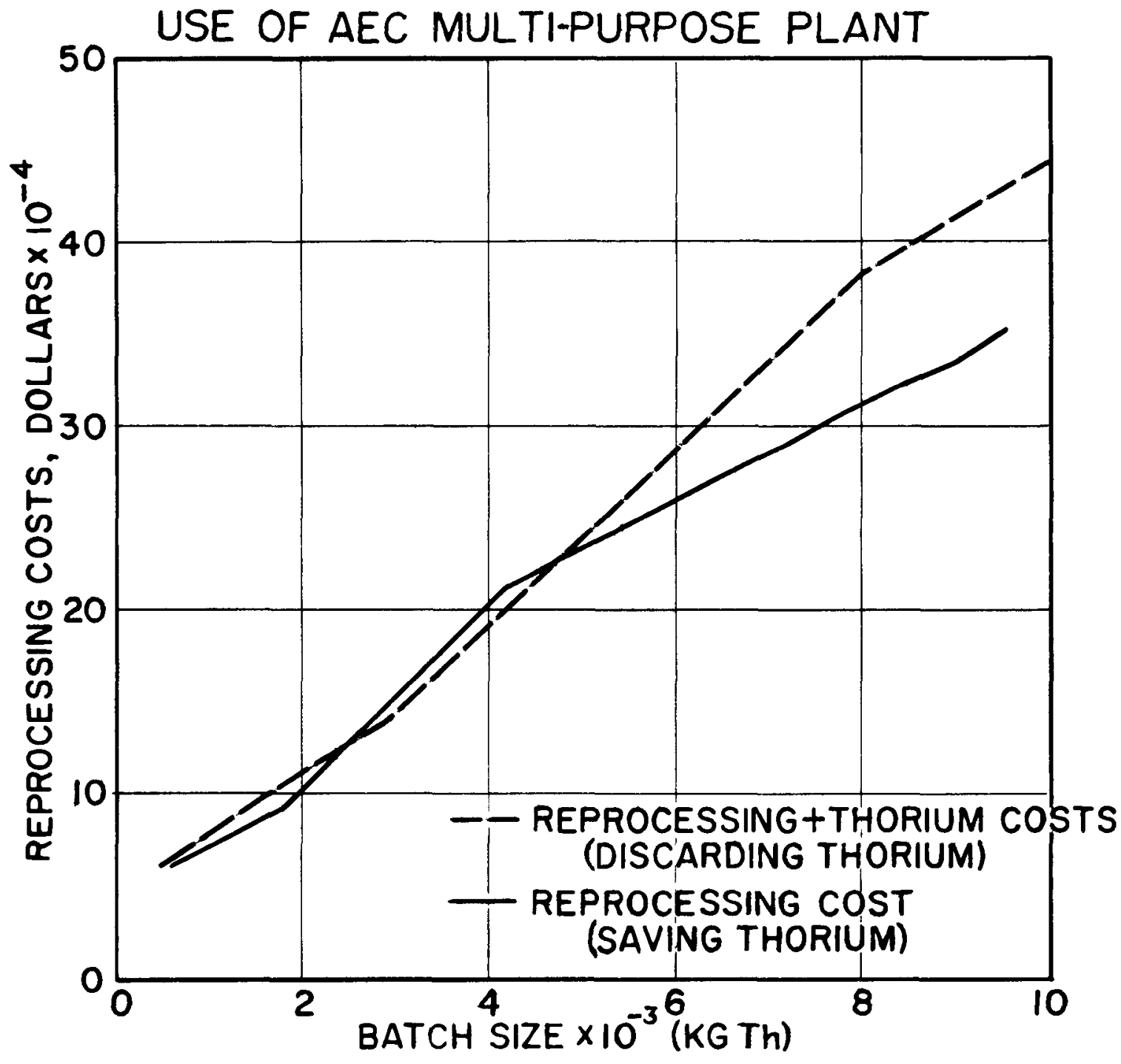


In order to determine minimum cooling time, it is necessary to determine the amount of $\mathrm{Pa}-233$, i.e. potential U-233 present, as a function of fertile material usage time. Assuming all $\mathrm{Pa}-233$ formed eventually goes $U-233$, it follows that:

$$
\frac{\mathrm{N}^{13} \text { present at time }(\mathrm{t})}{\text { Total } \mathrm{N}^{13} \text { formed until time }(\mathrm{t})}=\frac{\mathrm{C}\left(1-\mathrm{e}^{-\lambda^{13} \mathrm{t}}\right)}{\lambda 13(\mathrm{Ct})} \quad 3.1-6
$$

This function is presented in Figure 3.1-2. Using this relationship, it is possible to determine the optimum core or blanket cooling time as the time when the incremental inventory costs (after material is removed from reactor) equals the incremental value of $\mathrm{Pa}-233$ recovered.

$$
\begin{array}{ll}
\text { Inventory charges }=\frac{4 \times 10^{-2} \mathrm{VWt}}{365} & 3.1-7 \\
\text { Value of } \mathrm{Pa}^{233}=10^{-2} \mathrm{v} \% \text { APt } G_{n} e^{-\lambda^{\prime 3} t_{c}} & 3.1-8
\end{array}
$$

The sum of these two items can be considered to be the costs associated with cooling or:

$$
\left[\begin{array}{l}
\text { Costs associated } \\
\text { with cooling }
\end{array}\right]=1.1 \times 10^{-4} v W t_{c}+10^{-2} v \% A P t G_{n} e^{-\lambda^{13} t_{c}} \quad 3.1-9
$$

The minimum in this equation, i.e. the optimum cooling time, is found by the usual precedures to be:

$$
e^{-\lambda^{13} t_{c}}=\frac{1.1 \times 10^{-2} W}{\% \lambda^{13} A P t G_{n}} \quad 3.1-10
$$

Actually the optimum was found to be extremely flat and a deviation of as much as 30 days on either side of the minimum has no significant effect on fuel cycle costs.

Another factor in fuel cycle costs which was analyzed is the ratio of core to blanket reprocessing rates. Since the cost of manufacturing the blanket balls is not insignificant, one might conclude that the blanket use charge would continue to decrease with increasing blanket lifetime. This is not so due to the fact that holding up fissile material in the blanket, results in inventory changes on that portion of $U-233$ in the core that 
FIG.3.I-2 POTENTIAL FUEL PRESENT (\% $\left.\mathrm{Pa}^{233}\right)$

IN TOTAL BRED MATERIAL $\left(\mathrm{Pa}^{233}+\mathrm{U}^{233}\right)$

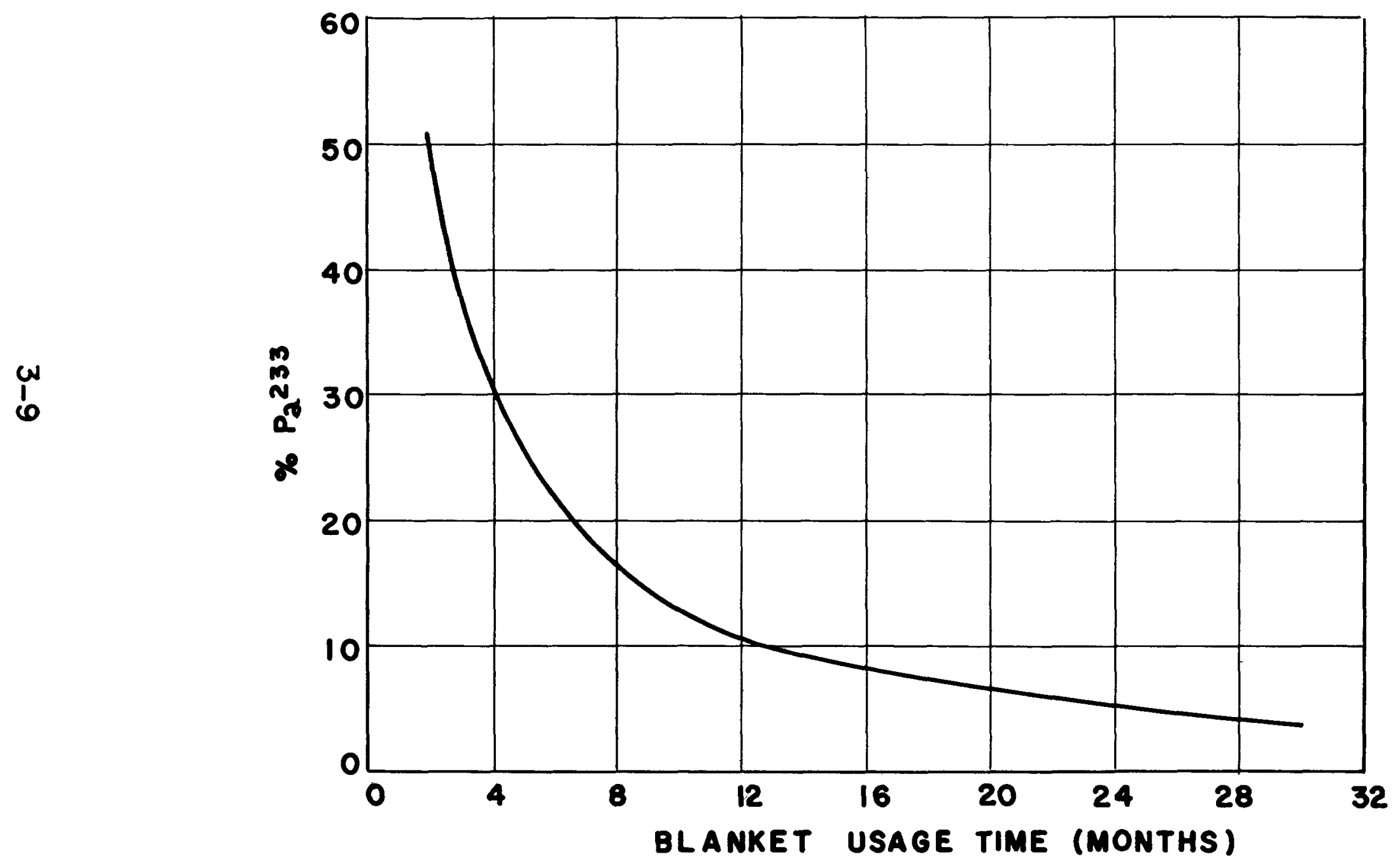


could be replaced by $U-233$ bred in the blanket. Therefore, the unit cost paid for recovered blanket $U-233$ (which includes $4 \%$ on the value of blanket U-233, reprocessing, and refabrication costs) as a function of blanket lifetime exhibits a minimum since the first item increases while the last two decrease.

Blanket U-233 inventory charge is a function of the rate at which U-233 accumulates in the blanket. This rate is expressed as follows:

$$
\mathrm{U}-233 \text { accumulation in blanket }=\mathrm{APG}_{\mathrm{b}} \mathrm{t}
$$

Since $G_{b}$ is dependent on blanket thorium concentration, and since blanket thorium concentration decreases with time, $G_{b}$ must be related to blanket usage time. Assuming that a differential change in the thorium concentration, for a given geometry and flux distribution, produces a proportionate change in the breeding ratio, it follows that:

$$
\frac{d G_{b}}{G_{b}}=\frac{A P G_{0} d t}{x_{0}} \quad 3.1-12
$$

and

$$
G_{b}=G_{0} e^{-\frac{A P G_{0} t}{X_{0}}} \quad 3.1-13
$$

Evaluation of eq. (3.1-13) for the range of thorium concentrations and times considered shows that $G_{b}$ is nearly constant and may be assumed equal to $\bar{G}_{b}$ for all practical purposes. Therefore eq. (3.1-11) becomes:

$$
\mathrm{U}-233 \text { accumulation in blanket }=\operatorname{AP} \overline{G_{b}} t \quad 3.1-14
$$

If the blanket is used for a time ( $t)$, the interest charge on the $\mathrm{U}-233$ value which has been accumulated over this time is given by:

$$
\text { Inventory cost on blanket }=\frac{.04 \text { VAP } \overline{G_{b}} t^{2}}{2} \quad 3.1-15
$$

For purposes of determining optimum blanket usage time, a constant cooling time of 130 days was used. The total blanket inventory charge per core is then the sum of the inventory charge based on the accumulated U-233 from previous cycles, the inventory charge based on U-233 accumulated during this cycle, and the inventory charge while a portion of the blanket equal to $1 / S$ is cooling and being reprocessed. 
The total blanket cost (manufacturing, reprocessing, and inventory charges) is shown in Figure 3.1-3 as a function of blanket usage time. It can be seen that an extremely flat minimum exists at about 6 years. Such a long blanket usage period cannot be recommended at this time because of the uncertainties of blanket stability and because of the heat generation rate in the blanket after such a long exposure. It is also likely that the assumption of a linear blanket breeding gain with time would no longer be justifiable. 
FIGURE 3.1-3

COST OF $U^{233}$ BRED IN BLANKET OF FINAL DESIGN

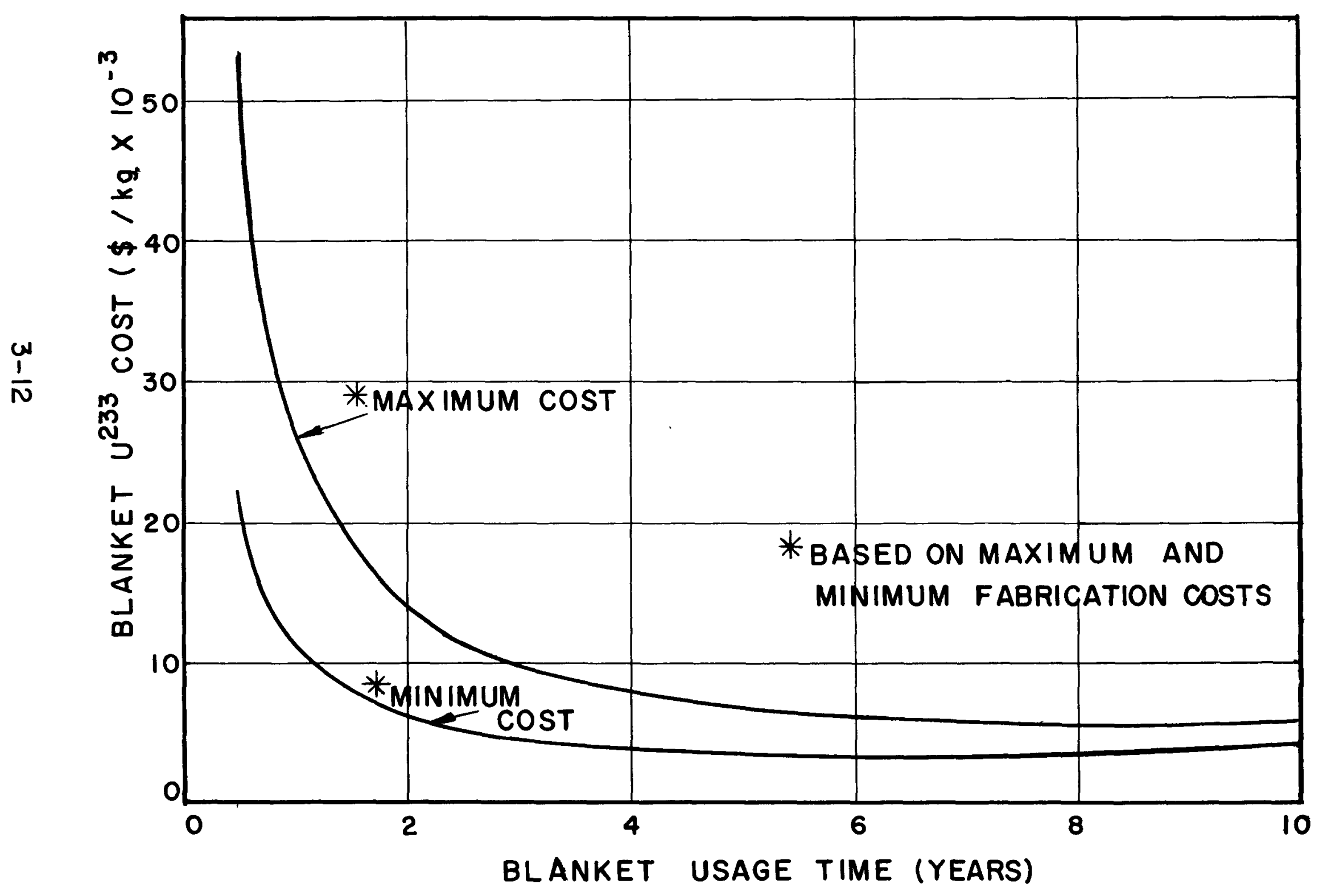




\section{2 Results of Analysis}

\subsection{Survey Cores}

The nuclear analysis, reported in Section 1, Part II, covers a range of cores of 7 to 20 feet in diameter. Figure 1.2-29 in Part II presents certain results of this survey, before Doppler broadened cross-sections and self-shielding corrections were applied. Using these data for qualitative comparisons in order to illustrate the dependency of fuel cycle costs on core size, three cores were selected for study, as listed in Table 3.2-1.

TABLE $3.2-1$

Cores Analyzed in Fuel Cycle Economic Study

\begin{tabular}{|c|c|c|c|c|c|c|}
\hline \multirow{3}{*}{$\begin{array}{l}\text { Core } \\
\text { Dia. } \\
\text { (Ft.) } \\
\end{array}$} & \multirow{3}{*}{$\begin{array}{c}\mathrm{U}-233 \\
\text { In Core } \\
(\mathrm{Kg} \cdot) \\
\end{array}$} & \multirow{3}{*}{$\begin{array}{c}\mathrm{Th} / \mathrm{U} \\
\text { Ratio } \\
\text { In Core } \\
\end{array}$} & \multirow{3}{*}{$\begin{array}{c}\mathrm{Th} \text { in } \\
\text { Blanket } \\
(\mathrm{Kg} \cdot) \\
\end{array}$} & \multicolumn{2}{|c|}{ Breeding Ratio } & \multirow{3}{*}{$\begin{array}{l}\text { Core } \\
\text { Life } \\
\text { (mo.) }\end{array}$} \\
\hline & & & & & & \\
\hline & & & & Core & Blanket & \\
\hline 9 & 85 & 9.8 & 11,460 & 0.51 & 0.34 & $3-1 / 3$ \\
\hline 16 & 232 & 21.8 & 33,538 & 0.745 & 0.185 & 9 \\
\hline 20 & 375 & 25.5 & 51,325 & 0.81 & 0.135 & 12 \\
\hline
\end{tabular}

These cores all had $25 \%$ voids, 10 weight percent of metallic oxide in the core elements, a $\mathrm{K}_{\text {eff }}$ of 1.10 and are blanketed. Minimum fuel costs were used in the comparison.

As evidenced from Figure 3.2-1, fuel cycle costs decrease with decreasing core size and increasing blanket usage time. In order to achieve a fuel cost comparable to current fossile fuel costs the core should be of the order of 9 feet in diameter and the blanket usage time of the order of 2 to 4 years. Figure 3.2-2 presents a cross plot of these data with blanket usage time as the parameter, showing that there is little difference in fuel costs in the use of small diameter cores, i.e. of the order of 9 feet. 
FIGURE $3.2-1$

FUEL CYCLE COSTS FOR CORES DESCRIBED IN TABLE 3.2-I

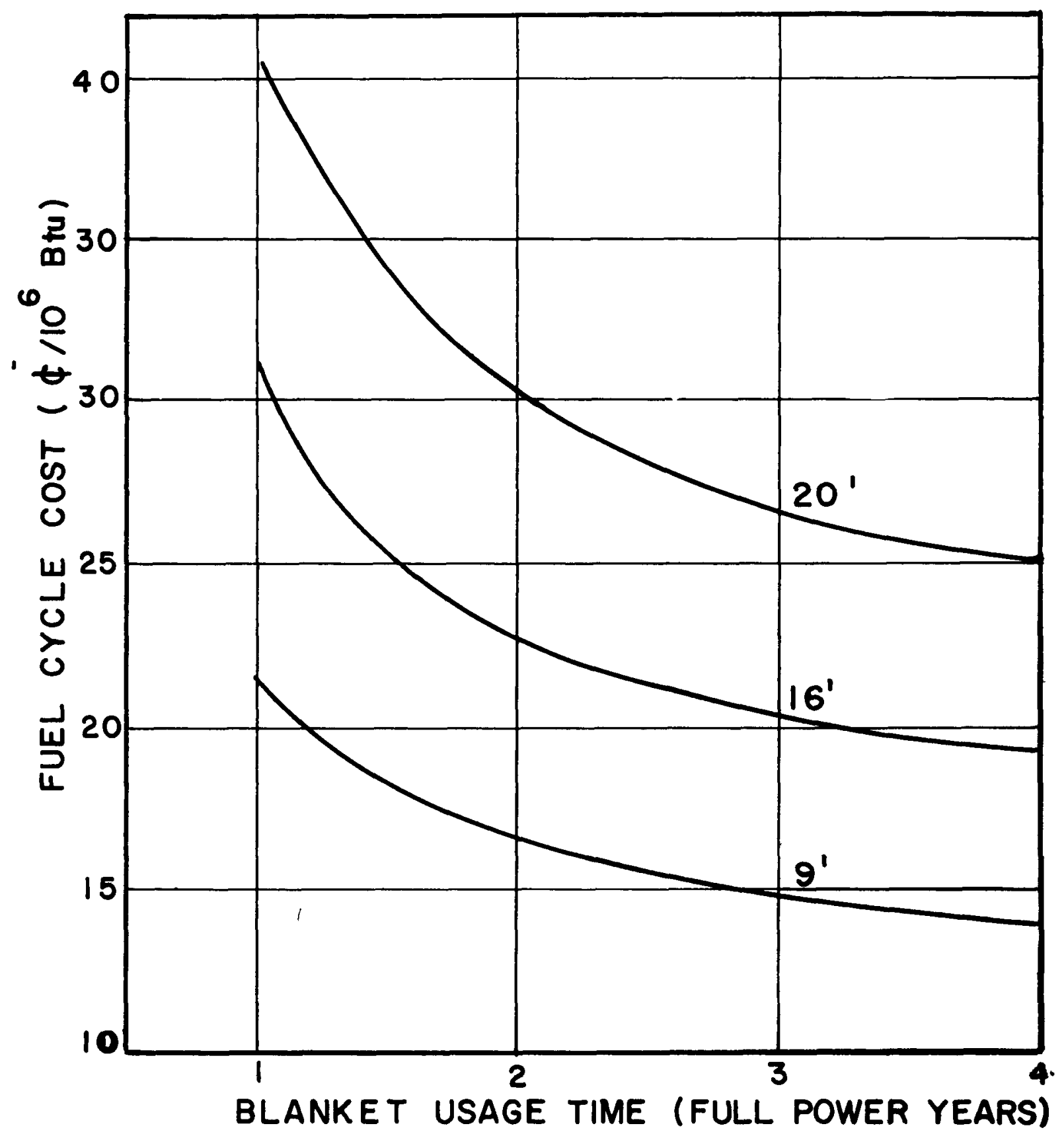


FIGURE 3.2-2

FUEL CYCLE COSTS FOR CORES

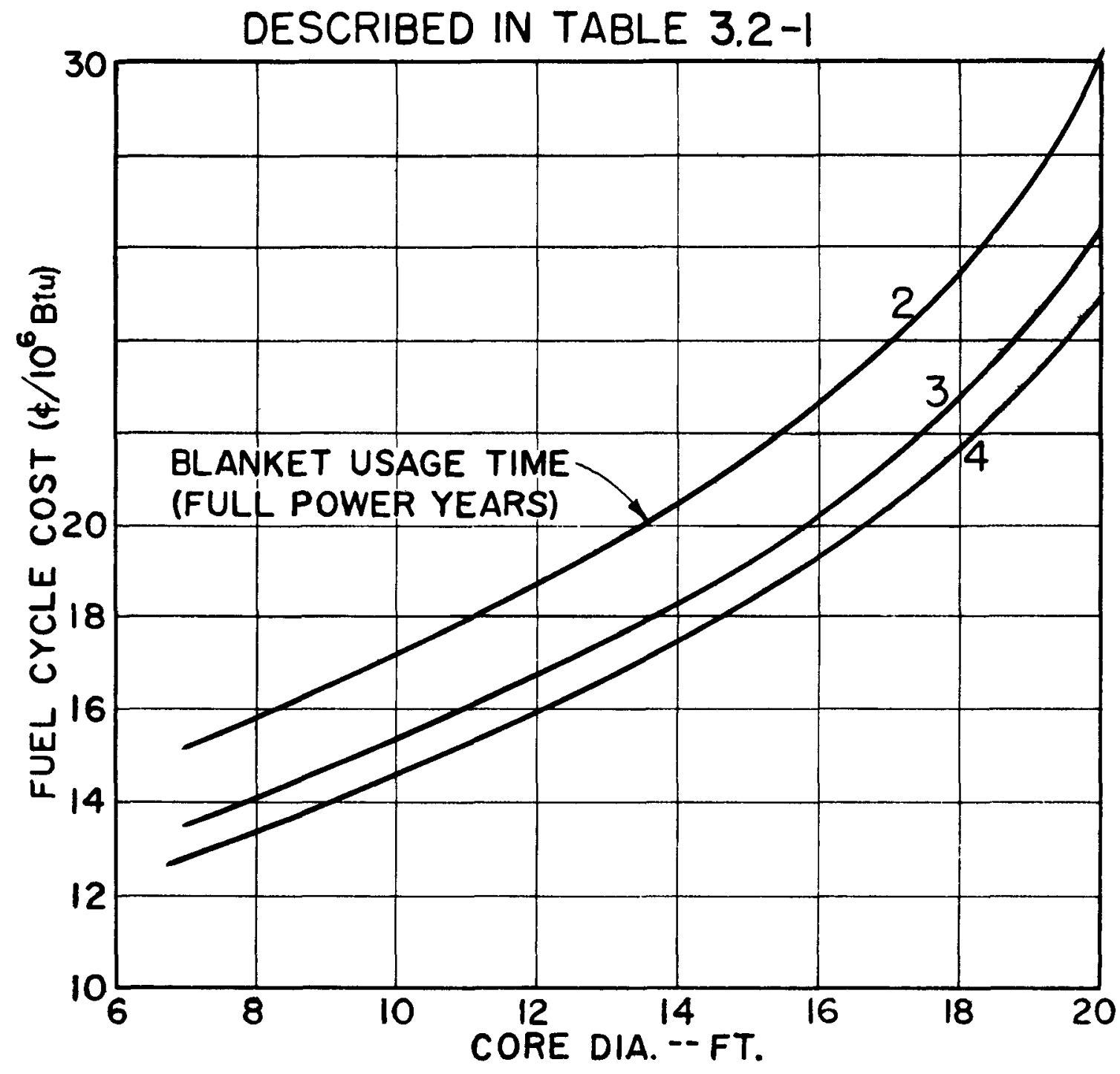

$3-15$ 


\subsubsection{Design Core}

From a consideration of the fuel cycle economics, plant economics as related to pumping power, and nuclear considerations, a blanketed core having a 9 foot diameter and $29 \%$ voidage was selected for final design. The reactor system has the following characteristics:

TABLE 3.2-2

$\begin{array}{lll}\text { Core } & \text { Blanket } \\ 383 & & 496 \\ 1-1 / 2 & & 3 / 4 \\ 597 & & 4780 \\ 228,600 & 2,370,000 \\ 11,085 & & 13,404 \\ 1,129 & & 13,404 \\ 102.6 & & - \\ 992.2 & & 11,780 \\ 90.2 & & - \\ 0.510 & & 0.353 \\ & 0.863 & \\ & 0.48 & \\ & 100 & \\ & 125 & \end{array}$

Maximum and minimum fuel cycle costs are calculated using the ratio of blanket to core usage time (S) as the main variable. Fuel burnup is independent of $S$ while inventory charges, reprocessing costs and blanket element fabrication vary with $S$. Shipping charges have been assumed to be independent of $\mathrm{S}$.

\subsubsection{Fuel Burnup and Losses}

Fuel burnup is equal to:

$$
\begin{aligned}
& \operatorname{APt}\left(1-\bar{G}_{0}\right)=6.38 \mathrm{Kg} \\
& \text { where: } A=0.48 \mathrm{Kg} / \mathrm{MWYr} \\
& P=350 \mathrm{MW} \\
& \begin{aligned}
\mathrm{t} & =100 / 365=0.274 \text { year } \\
\overline{\mathrm{G}}_{\mathrm{O}} & =0.863
\end{aligned}
\end{aligned}
$$


This equation assumes that all Th-233 formed goes to U -233 .

The U-233 lost in reprocessing amounts to $1 \%$ of the thru-put and is equal to:

$$
.01(90.2-6.38)=0.838 \mathrm{Kg} \text {. }
$$

Total charges associated with burnup and losses are equal to:

$$
\$ 15,000(6.38+0.838)=\$ 108,000
$$

It should be noted that these charges assume that equilibrium has been established insofar as the blanket breeding and reprocessing is concerned. This will not be so until $S$ cores have been reprocessed. During the time from initial startup until (S-1) cores have been reprocessed, only part of the fuel bred in the blanket in any one cycle will be recovered during reprocessing. The balance will be building up in the blanket. This has two adverse effects on fuel cycle economics, namely, 1) the burnup charge is higher during this period than after equilibrium is reached and 2) The inventory charge is higher throughout the entire blanket lifetime due to the fissile material being held in the blanket.

\section{2 .2 .2 Inventory}

Inventory costs are a function of the time involved in the fabrication of core elements, core lifetime, cooling and reprocessing and the ratio of blanket to core usage time, (S). Core lifetime has been set at 125 days, assuming an $80 \%$ load factor. Fabrication time is assumed to be half of this, based on fuel balls being fabricated at a rate of about 2500 per day and the full core being ready at the reactor site about a week before it is needed. Reprocessing time is a variable, being a function of $S$, but can be included in a cooling time of 130 days since \pm 30 days in cooling time means little as regards determining the optimum cost. The sum of these three periods is 317.5 days which has been rounded off to 320 days for all cases.

It is assumed that a fraction of the blanket, equal to $1 / \mathrm{S}$, is dropped each time the core is refueled. On this basis, the average amount of $\mathrm{U}-233$ in the blanket during any one core life is equal to:

$$
\left(\frac{S+1}{2}\right) \times\left(A P \bar{G}_{b} t\right)
$$


Therefore, inventory costs are equal to:

$$
\left[90.2+\frac{(S+1)}{2}\left(A P \bar{G}_{b} t\right)\right] \times \$ 15,000 \times .04 \times \frac{320}{365}
$$

\section{2 .2 .3 Reprocessing}

Reprocessing of the core and blanket is done together. The economics of the AEC multipurpose plant are such that reprocessing costs are based on the thorium content of the fuel mixture. The amount of thorium to be reprocessed is equal to that in the core and blanket, less the thorium burnup which is equal to:

$$
A P \bar{G}_{O t}=40.2 \mathrm{Kg}
$$

The amount of thorium to be reprocessed is equal to:

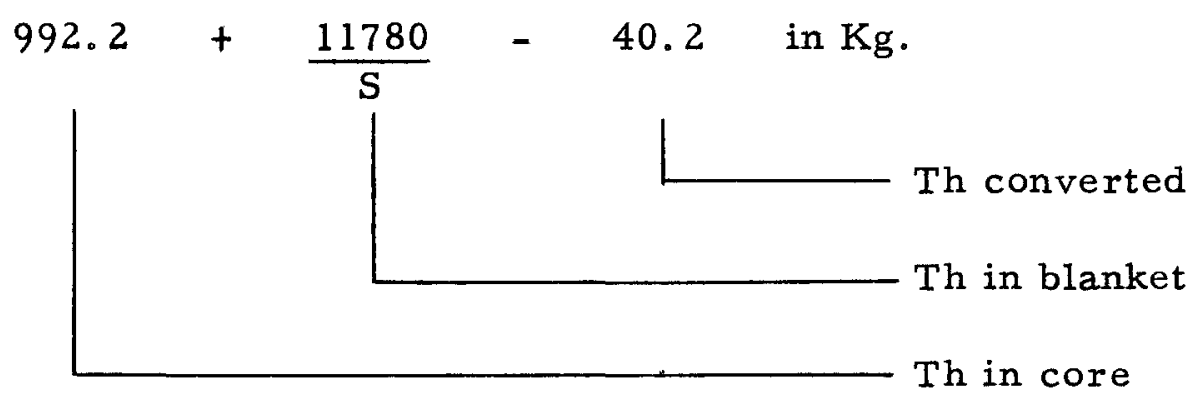

From inspection of Figure 3.1-1 it is seen that the reprocessing costs for saving or discarding thorium are essentially the same until a thru-put of $5000 \mathrm{Kg}$ per day is reached. Over the range of $\mathrm{S}$ values of interest, i.e. $S \geq 3$, the thorium to be reprocessed is less than $5000 \mathrm{Kg}$. Since both core and blanket balls have been quoted including thorium, it is assumed that the reactor charge will be reprocessed at the rate of $1000 \mathrm{Kg}$ per day and the thorium will be dircarded. Reprocessing costs are equal to:

$$
\$ 15,300\left(\frac{\mathrm{Kg} T h}{1000}+\text { turn-around time }\right)
$$

\subsubsection{Core and Blanket Element Fabrication}

Core fabrication costs are constant regardless of the value of $S$. Minimum and maximum prices of fuel balls, including thorium but less fissile material are $\$ 0.37$ and $\$ 0.76$. Since 229,000 balls are required for the core, the minimum core costs are $\$ 84,600$ and $\$ 174,000$ respec- 
tively.

Blanket ball fabrication costs per core loading vary inversely with S. Minimum and maximum unit prices of blanket balls are $\$ 0.165$ and $\$ 0.55$. Since $2,370,000$ balls are required for the blanket, the minimum and maximum costs are $\$ 390,000$ and $\$ 1,300,000$ for the total blanket. Costs associated with a core loading are 1/S of these costs.

\subsubsection{Shipping Costs}

Shipping costs are assumed constant, regardless of $S$, at $\$ 28,700$ minimum and $\$ 43,000$ maximum.

\subsubsection{Fuel Cycle Costs}

These costs are summarized in Table 3.2-3 over a range of $S$ from 6 to 16, or a blanket lifetime from 600 to 1600 full power hours, for maximum and minimum core and blanket fabrication and shipping costs. Fuel costs in $k / 10^{6} \mathrm{BTU}$ are equal to:

$$
\frac{\text { Fuel Cycle Costs } \times 10^{2}}{2.87 \times 10^{6}}
$$

The contribution of fuel cycle costs to production costs in mills/KWHr are equal to:

$$
\frac{\notin / 10^{6} \mathrm{BTU}}{10^{5}} \times \text { heat rate }
$$

The data summarized in Table $3.2-3$ is presented graphically in Figure 3.2.3. It is apparent that on the basis of minimum fuel costs a blanket to core life of about 8 is optimum. At the maximum level the optimum is somewhat beyond 16 although the cost curve is already quite flat at this point. Other factors that are affected by blanket lifetime such as the effect of a high $\mathrm{U}-233$ concentration on nuclear characteristics and the thermal performance of the blanket have not been evaluated.

To remain on the conservative side, a value of $S=10$ has been selected for use in calculating power costs for the reference design.

Figure 3.2-3 presents equilibrium fuel costs, that is fuel costs after $S$ core loadings have been effected. Figure 3.2-4 presents the increase in fuel costs from 1 to $S$ loadings, where $S=10$, over and above 
TABLE 3.2-3

Fuel Cycle Cost vs S

(\$ Costs in Thousands)

$\mathbf{S}$

Burnup \& losses - \$

Inventory - \$

Th reprocessed - Kg

Reproc. time - days

Reproc. costs - \$

Sub-total - \$
6

108.0

78.7

2914

5.9

90.2

276.9
8

108.0
87.6
2424
5.4
82.5
268.1
10

12

14

16

Min. Fuel Cycle Costs

Core - \$

Blanket - \$

Shipping - \$

Sub-total - \$

Total \$

$\notin / 10^{6} \mathrm{BTU}$

mills/KW Hr

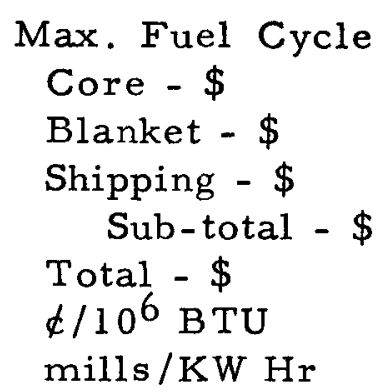

$\begin{array}{cccccc}84.6 & 84.6 & 84.6 & 84.6 & 84.6 & 84.6 \\ 65.0 & 48.8 & 39.0 & 32.5 & 27.9 & 24.4 \\ 28.7 & 28.7 & 28.7 & 28.7 & 28.7 & 28.7 \\ 178.3 & 162.1 & 152.3 & 145.8 & 141.2 & 137.7 \\ 455.2 & 430.2 & 434.8 & 434.2 & 436.9 & 440.9 \\ 15.85 & 15.0 & 15.1 & 15.2 & 15.3 & 15.4 \\ 1.44 & 1.36 & 1.37 & 1.38 & 1.39 & 1.40\end{array}$

174.0

174.0

174.0

174.0

174.0

174.0

217.0

163.0

130.0

108.0

93.0

81.0

43.0

43.0

43.0

43.0

43.0

43.0

434.0

710.9

380.0

347.0

325.0

310.0

$648.1 \quad 629.5$

613.4

605.7

298.0

24.8

22.6

21.9

21.4

21.1

601.2

2. 25

2.05

1.99

1.94

1.92

20.9

1.90 
FIGURE 3.2-3

EQUILIBRIUM FUEL CYCLE COSTS

VS.

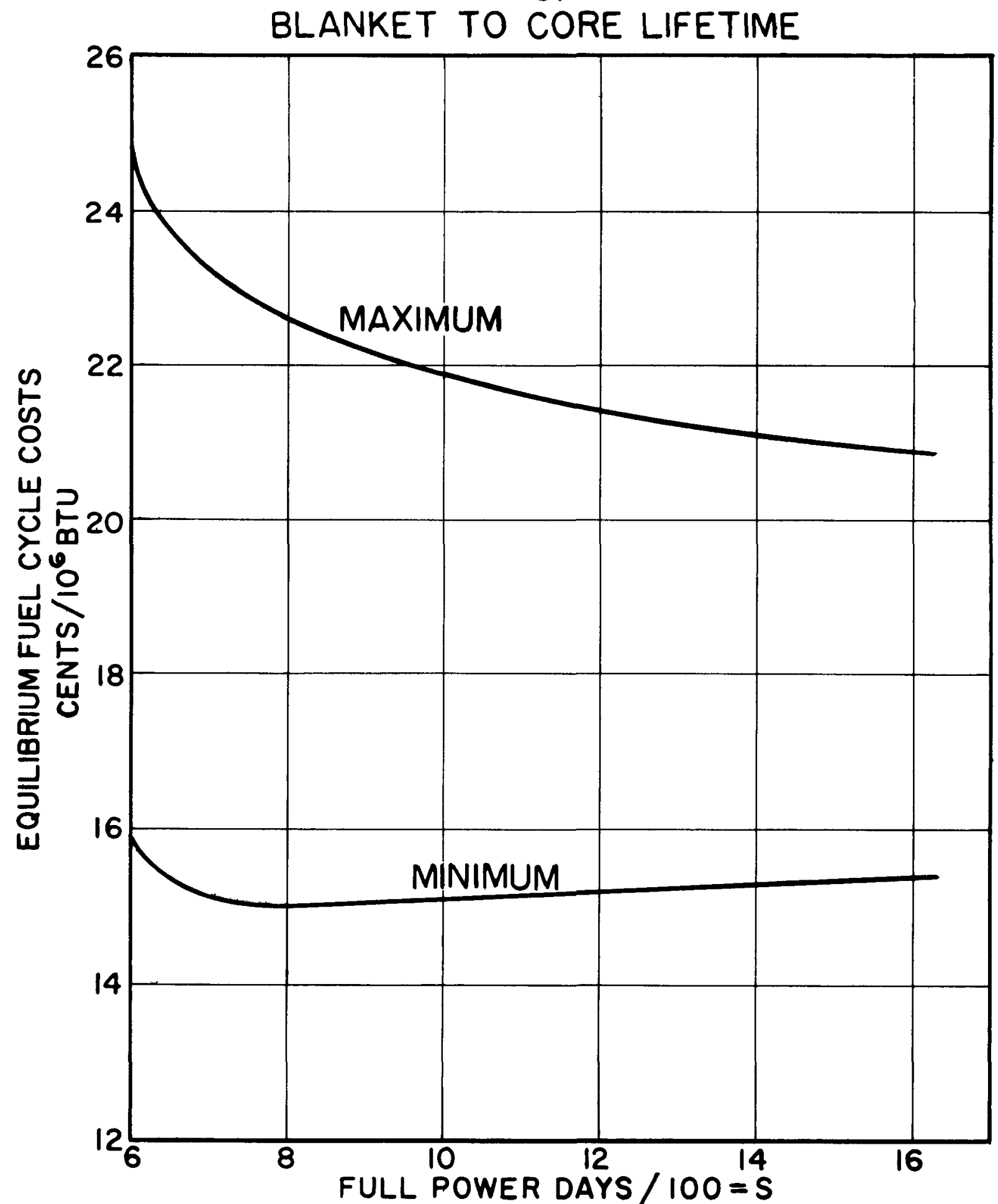


FIGURE 3.2-4

INCREMENTAL FUEL COSTS

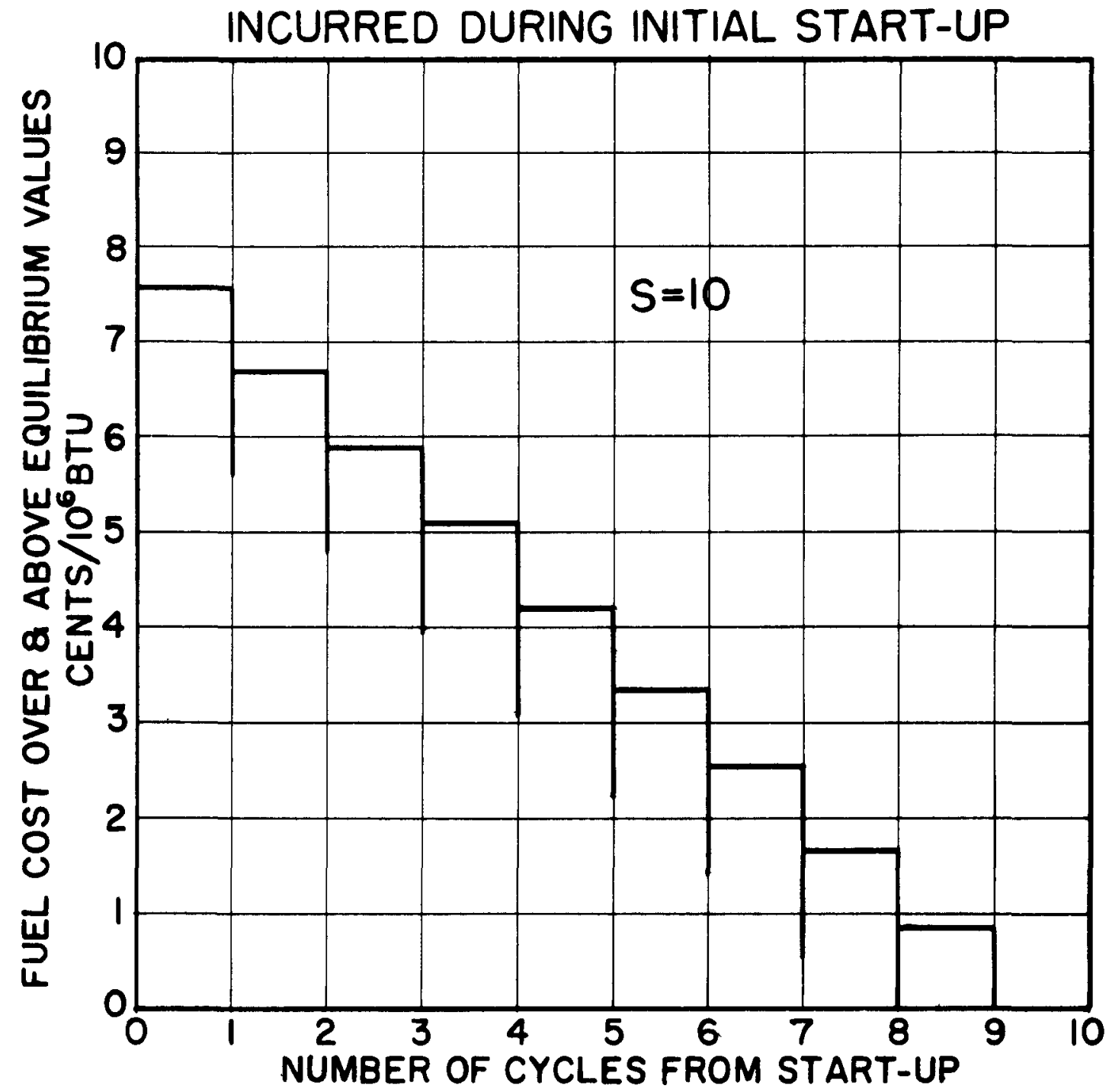


the data indicated in Figure 3.2-3. It should be noted that this added cost, which is incurred at the beginning of the operation, is completely recoverable at a time when the blanket is reprocessed in its entirety.

As noted in Section 2.1 of Part I, there is a question regarding the a values of U-233. Fuel cycle costs for the 125 eMW-PBR have been calculated using the conservative values of Figure 1.2-6 in Part II. Using the more optimistic values of a from this figure results in a reactor having the characteristics presented in Table 3.2-4.

Fuel cycle costs at $S=10$ have been calculated for this cycle. Minimum costs are $12.5 \mathrm{~d} / 10^{6} \mathrm{BTU}$. Maximum costs are $19.15 \mathrm{~d} / 10^{6} \mathrm{BTU}$.

TABLE $3.2-4$

Characteristics of Reactor Using Optimistics Breeding Ratio

Core Blanket

Ball bed volume

383

496

Element diameter, in.

Elements per $\mathrm{Ft}^{3}$

$1-1 / 2$

597

$3 / 4$

228,600

4780

Total elements

Composition by weight, Kg.

15,405

$1,498.0$

102.6

$1,316.9$

90.2

0.585
$\mathrm{ThO}_{2}$

$\mathrm{Th}-232$

$\mathrm{U}-233$

Breeding ratio (initial)

Total (initial)

Fuel burnup rate, (Kg/MWYr)

Core life at full power, days

Core life at $80 \% \mathrm{LF}$, days

$\mathrm{K}_{\text {eff }}$ (initial)
13,404

13,404

11,780

$0 . \overline{4} 50$
1.035

.434

130

156

1. 10

Fuel cycle costs have not been calculated for a core utilizing continuous or day by day reloading with on-site reprocessing and fabrication. Such a design has been evaluated insofar as the neutronics are concerned. This core would have a $\mathrm{Th} / \mathrm{U}$ ratio of $16: 1$. an overall conversion ratio of 0.97 and a $K_{\text {eff }}$ of 1.02 . On the assumption that fuel is reprocessed in the same manner as in the reference design and the core 
residence time is 5 months, minimum and maximum fuel costs are 8 and $14 \notin / 10^{6} \mathrm{BTU}$ respectively. In practice fuel would be reprocessed on the site at a much lower decontamination level and fuel balls would, in all probability, be fabricated by impregnation. Thus reprocessing costs would be reduced and shipping costs virtually eliminated.

Therefore it can be concluded that the effect of differences in a values of $\mathrm{U}-233$ have but a minor effect on fuel costs but that real gains can be achieved by developing a more or less continuous reloading cycle with on-site reprocessing and fabrication of fuel elements. 
$\mathrm{V}$ - Value of fully enriched U-233 $=\$ 15 / \mathrm{gram}$

$\mathrm{X}_{\mathrm{O}}$ - Initial thorium loading - $\mathrm{Kg}$

$\bar{G}_{a}$ - Average breeding ratio in the blanket

$G_{n} \quad$ - Breeding ration in designated section

A $\quad-0.48 \mathrm{Kg}$. U-233 per MWYr

$\mathrm{P}$ - Power level $=350 \mathrm{tMW}$

t - Time

$\mathrm{t}_{\mathrm{c}}$ - Cooling time

$\mathrm{t}_{1 / 2}$ - Half life

$\lambda^{1 / 2}$ - Decay constant

Superscripts 02, 03, 13, 23 refer to Th-232, Th-233, Pa-233 and U-233 respectively.

$\mathrm{N} \quad$ - Number of atoms

$\sigma_{\mathrm{a}}$ - Absorption cross-section

$\phi$ - Neutron flux

Subscripts th, f, refer to thermal and fast flux

$\%$ - Percent of bred material present as Pa-233

$\mathrm{W}$ - Kg of U-233 in material to be cooled

C - Constant

S - Ratio of blanket to core lifetime 
Section 4.0

Steam Cycle Analysis

4.1 Steam Conditions $\ldots \ldots \ldots \ldots \ldots \ldots \ldots \ldots \ldots \ldots \ldots \ldots \ldots$

4.2 Final Feedwater Temperature............ 4-2

4.3 Number of Feedwater Heaters ............ 4-7

4.4 Turbine Selection ................. 4-7 


\subsection{Steam Cycle Analysis}

\subsection{Steam Conditions}

For a given maximum reactor heat output, higher net thermal efficiencies can produce a greater net electrical generation and consequently higher economy of operation. The best thermal efficiencies in a steam power plant can be produced by going to more advanced designs such as supercritical pressures, higher temperatures, multiple stages of reheat and a large number of regenerative feedwater heaters. There can be no doubt that the Pebble Bed Reactor steam power plant concept can expect to benefit from improvements of thermal efficiencies by the adoption of these devices. However, in the interest of simplicity and reliability, it was decided to use a non-reheat turbine with more conventional pressures and temperatures for the first look at a nuclear power plant of this type.

Because of metalurgical considerations, it seems advisable to maintain the maximum reactor outlet temperature in the neighborhood of $1250^{\circ} \mathrm{F}$. Above this limit there is very little experience with high pressure equipment with the high load factor and long life that is required for power plant service. To allow a margin for operating difficulties and to provide for heat losses in the concentric pipe configuration adopted for high temperature helium lines, the normal maximum helium temperature entering the superheater was fixed at $1200^{\circ} \mathrm{F}$. Furthermore, a superheater hot end terminal temperature difference of $200^{\circ} \mathrm{F}$ was selected as providing a reasonable compromise between loss in cycle efficiency and excessive superheater surface requirements despite the fact that increasing superheater outlet steam temperature by $50^{\circ} \mathrm{F}$ could have improved the heat rate about $1 \%$. This fixes the maximum superheater outlet temperature at $1000^{\circ} \mathrm{F}$.

A steam pressure of 1450 psig was selected as optimum for the $1000^{\circ} \mathrm{F}$ steam temperature, since it is a conservative value and produces a net station heat rate in the order of $9100 \mathrm{BTU} / \mathrm{KWHr}$ at optimum final feedwater temperatures. This heat rate corresponds to better than $94 \%$ of the highest currently attainable thermal efficiencies. Increasing the steam pressure to 1800 psig can theoretically improve the net heat rate by approximately $1.2 \%$ but it has the disadvantage of increasing the moisture content of the steam in the last stages of the turbine, in a non-reheat cycle, and hence will tend to expose the turbine to erosion problems. 
In view of the above discussion steam conditions of 1450 psig/ $1000^{\circ} \mathrm{F}$ were selected for the purposes of this study, because the net heat rate produced is competitive with the most modern power plants, in addition to the fact that consideration is given to the simplest and most reliable power plant arrangement consistant with good practice in the production of low cost power.

\subsection{Final Feedwater Temperature}

In order to investigate the effects of final feedwater temperature, a study was made with the following variables held constant:

a. Reactor heat output - $350 \mathrm{MW}$

b. Reactor output temperature - $1200^{\circ} \mathrm{F}$

c. Minimum steam generator heat transfer temperature difference $-50^{\circ} \mathrm{F}$

d. Steam conditions - $1450 \mathrm{psig} / 1000^{\circ} \mathrm{F}$

e. Turbine exhaust pressure $-1.5^{\prime \prime}$ of $\mathrm{Hg}$. Abs .

f. Turbine type-Tandem compound, triple flow exhaust section with a last stage blade length of 23 inches.

g. Steam generator drum pressure - 1600 psia

Gross turbine heat rates were calculated for varying final feedwater temperatures in accordance with the above conditions and are shown on Figure 4.2-1. It was assumed that the turbine efficiency remained constant regardless of output. The effects of varying final feedwater temperature on electrical generation and auxiliary power are shown on Figures $4.2-2$, and $4.2-3$, respectively.

Figure 4.2-3 illustrates the fact that at a constant reactor output the helium blower pumping power increases with increasing final feedwater temperature. This increase in pumping power is due to $\mathrm{zn}$ increasing helium blower temperature as well as an increasing helium flow caused by a decreasing temperature rise through the reactor. The relationship between final feedwater temperature and pumping temperature is shown on Figure 4.2-4 for a steam generator minimum helium to steam temperature difference of $50^{\circ} \mathrm{F}$. In order to keep the reactor geometry fixed, the pumping power was adjusted for final feedwater temperature from the case calculated for the heat balance (as shown in Part I, Section 4.0), in accordance with the postulate that pumping power is directly proportional to the product of the cube of the thermal heat generated in the reactor, the absolute pumping temperature, and the mean reactor temperature, and inversely pro- 
FIG. $4.2-1$

GROSS AND NET HEAT RATES VS. FINAL

FEEDWATER TEMPERATURE

\section{REACTOR OUTPUT $=350 \mathrm{MW}$}

MIN. HELIUM - STEAM $\triangle T=50^{\circ} \mathrm{F}$

MAX. REACTOR OUTLET TEMP. $=1200^{\circ} \mathrm{F}$

TURBINE TYPE - TC 3F-23"

STEAM CONDITIONS:

SUPERHEATER OUTLET PRESS. $=1465$ PSIA

SATURATION PRESS. = 1600 PSIA
SUPERHEATER OUTLET TEMP. = $1000^{\circ} \mathrm{F}$

TURBINE EXHAUST PRESS. = I.5" HG ABS

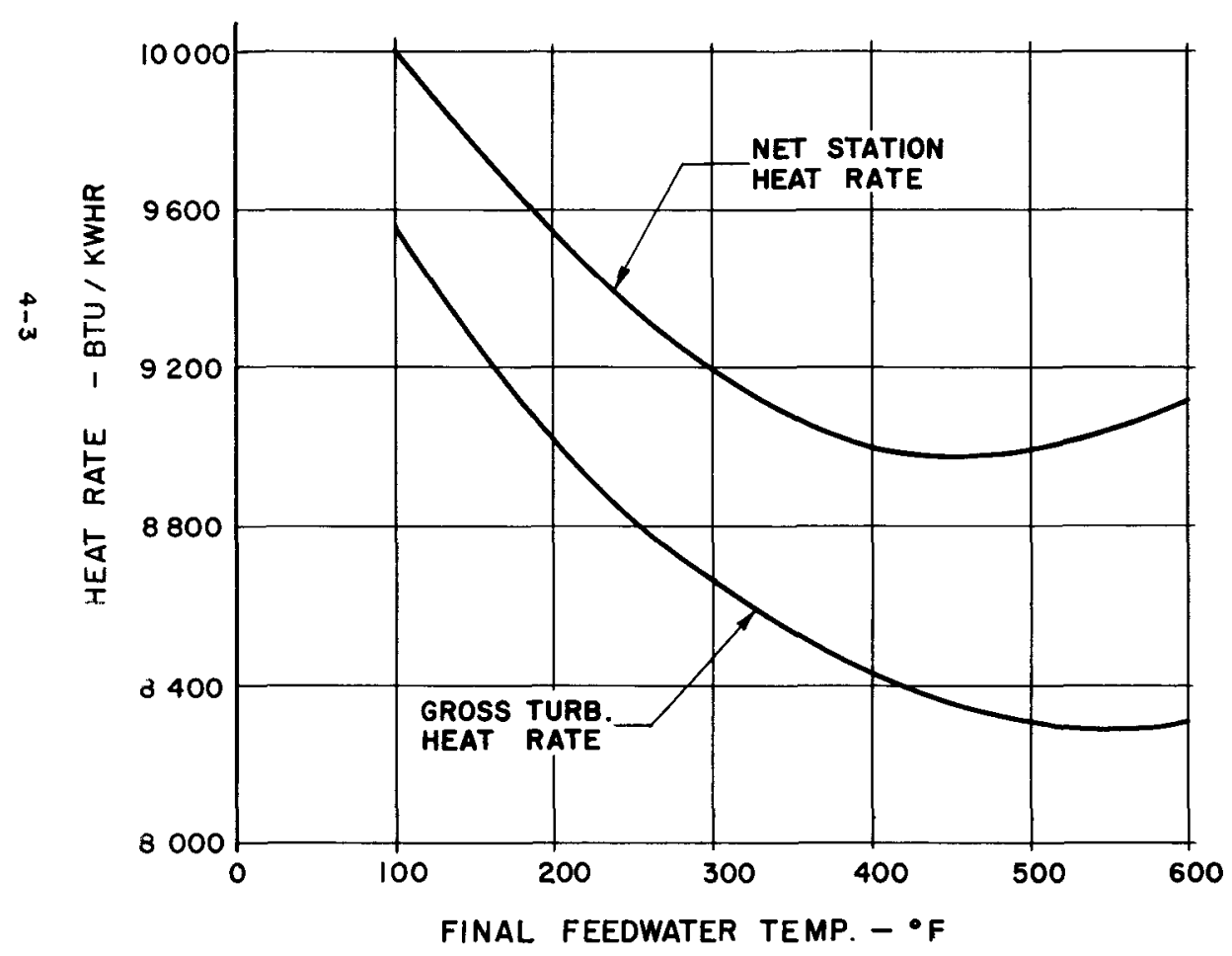

FIG. $4.2-2$

GROSS AND NET ELECTRICAL GENERATION VS. FINAL FEEDWATER TEMPERATURE

\section{REACTOR OUTPUT $=350 \mathrm{MW}$}

MIN. HELUUM - STEAM $\triangle T=50^{\circ} \mathrm{F}$

MAX. REACTOR OUTLET TEMP. $=1200^{\circ} \mathrm{F}$

TURBINE TYPE - TC 3F-23"

STEAM CONDITIONS:

SUPERHEATER OUTLET PRESS. $=1465$ PSIA

SATURATION PRESS. = 1600 PSIA

SUPERHEATER OUTLET TEMP. $=1000^{\circ} \mathrm{F}$

TURBINE EXHAUST PRESS. $=1.5 " \mathrm{HG}$ ABS

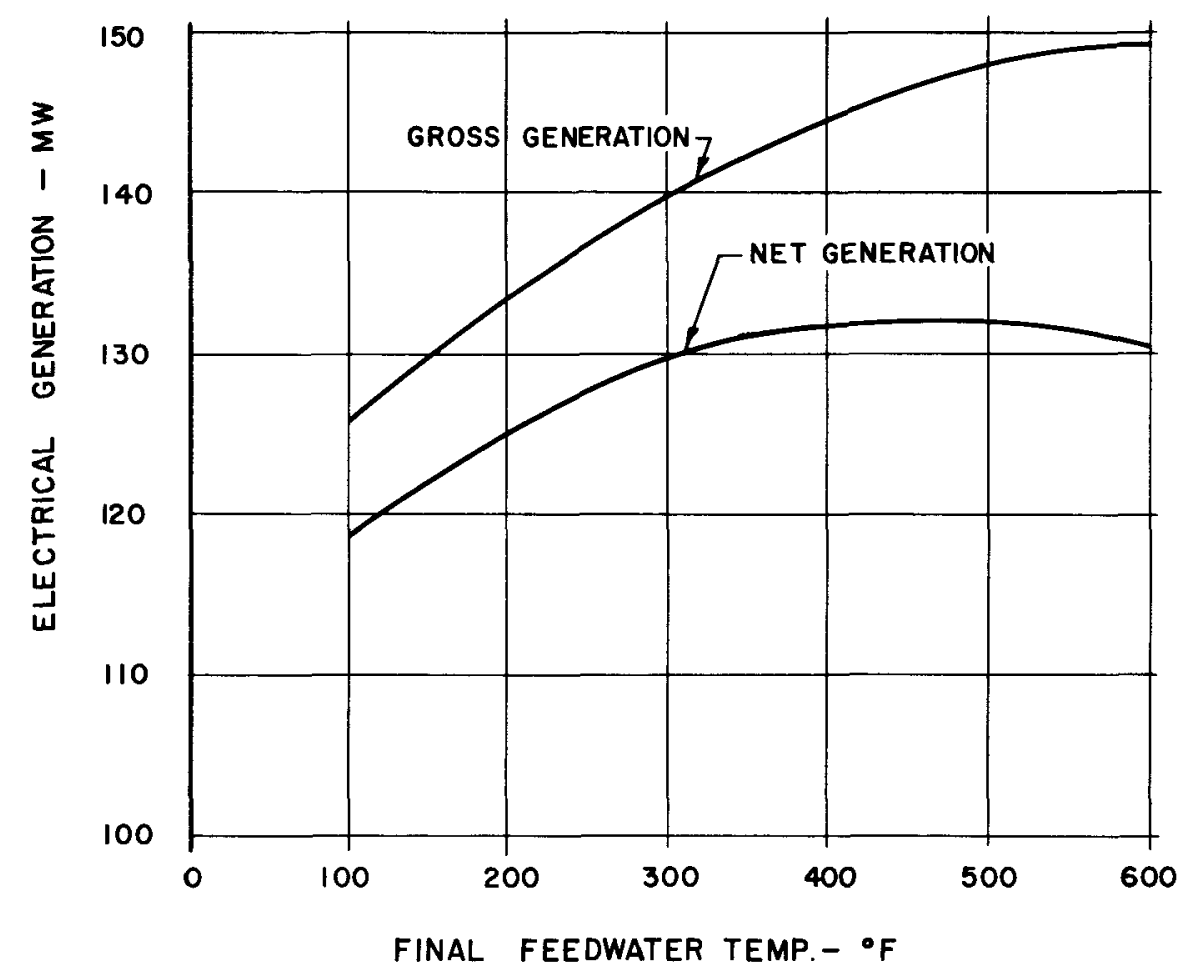


FIG. $4.2-3$

AUXILIARY POWER REQUIREMENTS VS.

FINAL FEEDWATER TEMPERATURE

REACTOR OUTPUT = $350 \mathrm{MW}$

MIN. HELUM - STEAM $\triangle T=50^{\circ} \mathrm{F}$

MAX. REACTOR OUTLET TEMP. $=1200^{\circ} \mathrm{F}$

TURBINE TYPE - TC 3F-23"

STEAM CONDITIONS :

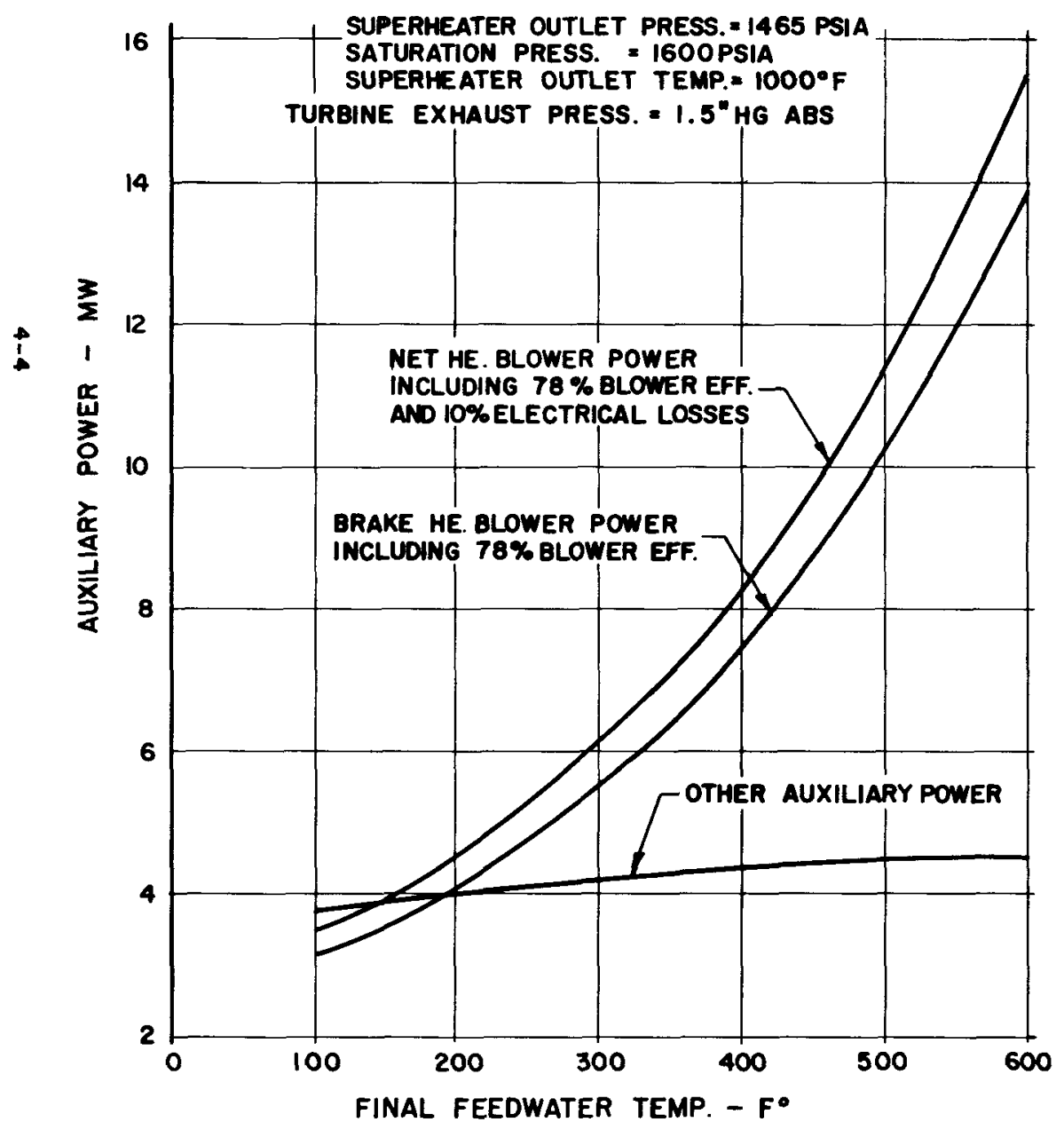

FIG. 4.2- 4

HELIUM BLOWER INLET TEMPERATURE VS. FINAL FEEDWATER TEMPERATURE

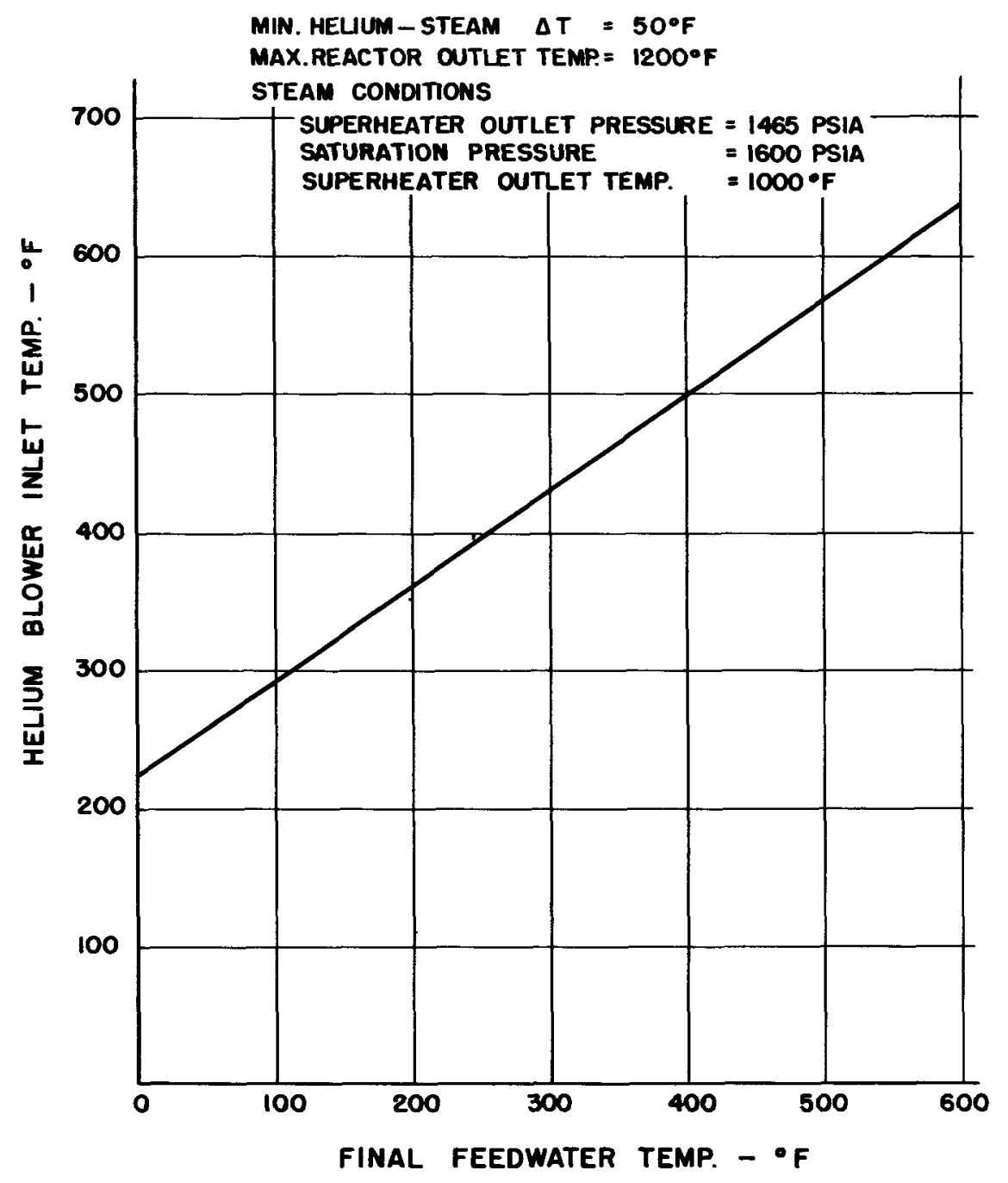


portional to the square of the reactor temperature rise. The helium blower inlet temperature was assumed to be equal to the reactor inlet temperature in order to simplify the calculations, for this study. The steam generator output at all conditions is the sum of the reactor output and the helium blower pumping power, neglecting heat losses. Therefore the steam generator output increases slightly with increasing feedwater temperature due to the fact that pumping power increases while reactor output is assumed constant.

It'can be seen from Figure 4.2-2 that the gross electrical generation increases with increasing final feedwater temperature, since it benefits from both increasing steam generator output and decreasing gross turbine heat rate as feedwater temperatures are increased. However, the net electrical generation becomes a maximum between $400^{\circ} \mathrm{F}$ and $500^{\circ} \mathrm{F}$, due to the fact that it is the difference between the gross electrical generation and the auxiliary power. The auxiliary power, including the helium blower power, is increasing more rapidly than gross generation as the final feedwater temperature becomes higher, causing the net generation to reach a maximum value. Since the net station heat rate is the ratio of the reactor heat output ( $350 \mathrm{nw}$ ) and the net generation, it becomes a minimum between final feedwater temperatures of $400^{\circ} \mathrm{F}-500^{\circ} \mathrm{F}$ (Figure $4.2-1$ ), because the net generation is at a maximum value at this point.

To obtain a valid comparison of the various feedwater temperatures, it is necessary to add quantitative economic factors into the picture. In order to accomplish this, a total erected plant cost of $\$ 33,000,000$ was assumed for a final feedwater temperature of $400^{\circ} \mathrm{F}$. This is based upon preliminary estimates for this facility of $\$ 265 / \mathrm{KW}$ and is within approximately $5 \%$ of the estimated cost of the facility as shown in Part II, Section 5 of this report. Adjustments to total plant cost due to the effect of varying the final feedwater temperature were made for gross capability, steam generator and feedwater heater surface, and auxiliary power, producing total plant cost as shown on Figure 4.2-5. Since the capital charge of producing electrical energy is directly proportional to the capital cost and inversely proportional to the net generation (at a constant fixed charge and load factor), this value becomes a minimum near the point where the net generation is a maximum, because the capital costs are relatively constant. This point is illustrated on Figure 4.2-6 at zero fuel cost. A charge of $1 \mathrm{mill} / \mathrm{KW} \mathrm{Hr}$ is added to the capital charges to cover estimated operating and maintenance costs. 
FIG. 4.2-6

FIG. 4.2-5

RELATIVE CAPITAL COST OF PEBBLE BED REACTOR STEAM POWER PLANT VS. FINAL FEEDWATER TEMP.

REACTOR OUTPUT $=350 \mathrm{MW}$

MIN. HELIUM - STEAM $\triangle T=50^{\circ} \mathrm{F}$

MAX. REACTOR OUTLET TEMP. $=1200^{\circ} \mathrm{F}$

TURBINE TYPE $=$ TC $3 F-23^{*}$

STEAM CONDITIONS

SUPERHEATER OUTLET PRESS. $=1465$ PSIA

SATURATION PRESS $=1600$ PSIA

SUPERHEATER OUTLET TEMP. = $1000^{\circ} \mathrm{F}$

TURBINE EXHAUST PRESS. $=1.5^{\circ} \mathrm{HG}$ ABS

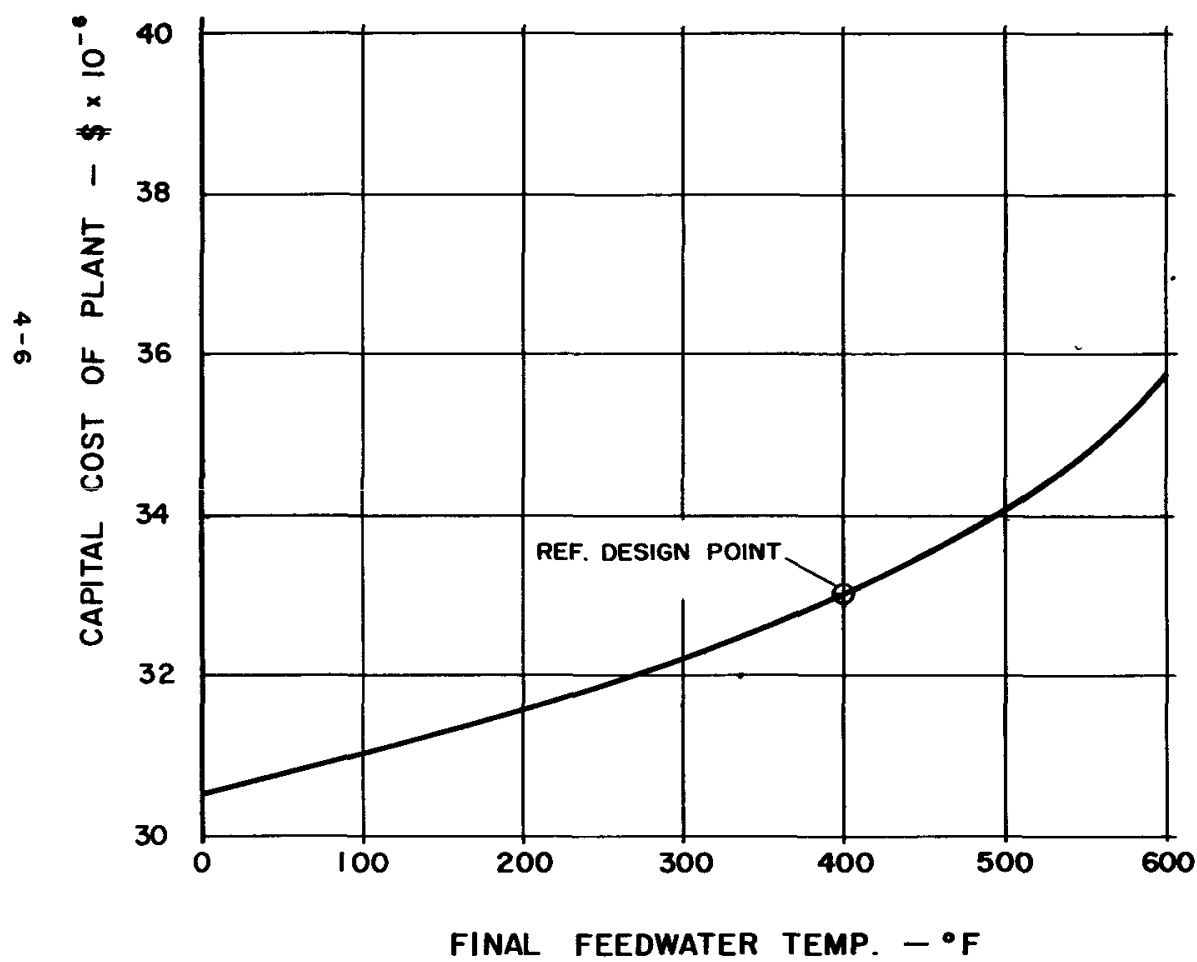

TOTAL COST OF PRODUCING ENERGY VS. FINAL

FEEDWATER TEMPERATURE AT VARIOUS FUEL COSTS

REACTOR OUTPUT $=350 \mathrm{MW}$

MIN HELIUM - STEAM $\triangle T=50^{\circ} \mathrm{F}$

MAX REACTOR OUTLET TEMP. $=1200^{\circ} \mathrm{F}$

TURBINE TYPE TC 3F-23"

STEAM CONOITIONS:

SUPERHEATER OUTLET PRESS $=1465$ PSIA

SATURATION PRESS. $=1600$ PSIA

SUPERHEATER OUTLET TEMP $=1000^{\circ} \mathrm{F}$

TURBINE EXHAUST PRESS. $=1.5^{\prime \prime}$ HG ABS

OPERATING AND MAINTAINANCE CHARGES= | MILL / KWHR LOAO FACTOR $=80 \%$

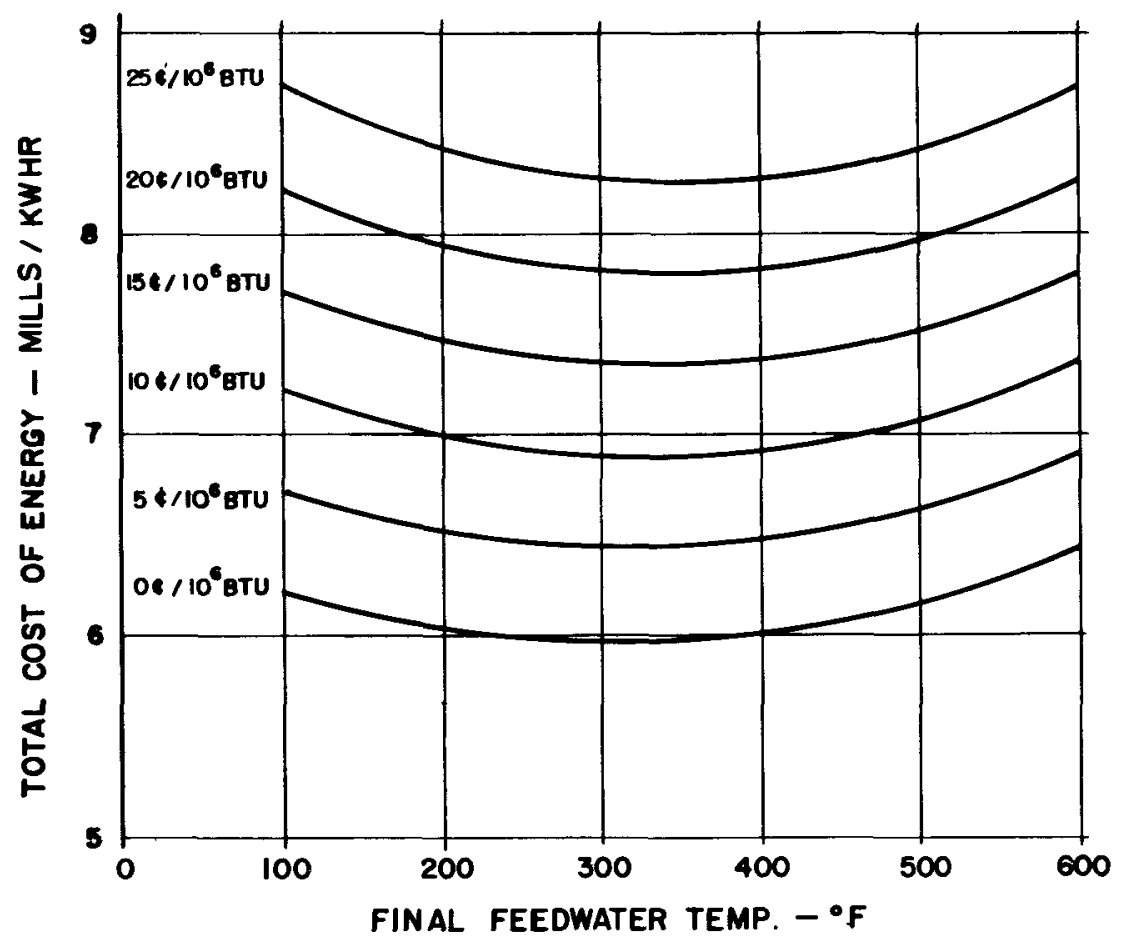


The fuel cost associated with the pebble bed reactor concept are in the order of $\$ .10$ to $\$ .15$ per million BTU's as indicated in Section 3.0. Since this order of magnitude of fuel costs produces a fuel charge between 1 and $1-1 / 2$ mills/KW $\mathrm{Hr}$ at a net heat rate of $10,000 \mathrm{BTU} / \mathrm{KW}$ $\mathrm{Hr}$, it is evident that the fuel costs will be a relatively small factor in the determination of final feedwater temperature, due to the fact that the fuel charges only constitute about $15 \%$ of the total cost of energy. However, at a constant fuel cost, the fuel charge becomes a minimum at a point where the net heat rate also is at a minimum value. The total cost of producing electrical energy is illustrated on Figure 4.2-6 at total fuel costs of zero, \$.05, \$.10,\$.15, \$.20, and \$.25 per million BTU. The minimum cost of producing electricity occurs between $350^{\circ}$ and $400^{\circ} \mathrm{F}$ at all of the fuel costs that were evaluated.

In view of the above discussion, a final feedwater temperature of $400^{\circ} \mathrm{F}$ was chosen as optimum, since the total cost of producing electrical energy is near a minimum value for this feedwater temperature regardless of the fuel operating at costs. Although this feedwater temperature is slightly on the high side of the optimum for maximum plant capability, a small reduction in load will tend to drop the final feedwater temperature toward the optimum and the overali plant operating efficiency will be at a higher level.

\section{3 Number of Feedwater Heaters}

The selection of the number of stages of regenerative feedwater heaters for the steam cycle is based qualitatively on Figure 4.3-1, which shows the maximum reduction in heat rate for various numbers of stages of regenerative feedwater heaters. It can be seen by inspection of Figure 4.3-1 that four stages of feedwater heating will be close to the optimum and therefore, this was adopted for the purpose of this design.

\subsection{Turbine Selection}

For a non-reheat turbine of $125 \mathrm{MW}$ size and steam conditions of $1450 \mathrm{psig}$ and $1000^{\circ} \mathrm{F}$, a $3600 \mathrm{rpm}$ tandem compound turbine is the natural choice. However, it becomes necessary to justify the selection of an exhaust section.

In this analysis, only a $23 "$ last stage blade length was considered, because preliminary inspection indicated that shorter blade lengths in- 
FIG. 4.3-1

REDUCTION IN HEAT CONSUMPTION OBTAINED BY REGENERATIVE FEEDWATER HEATING

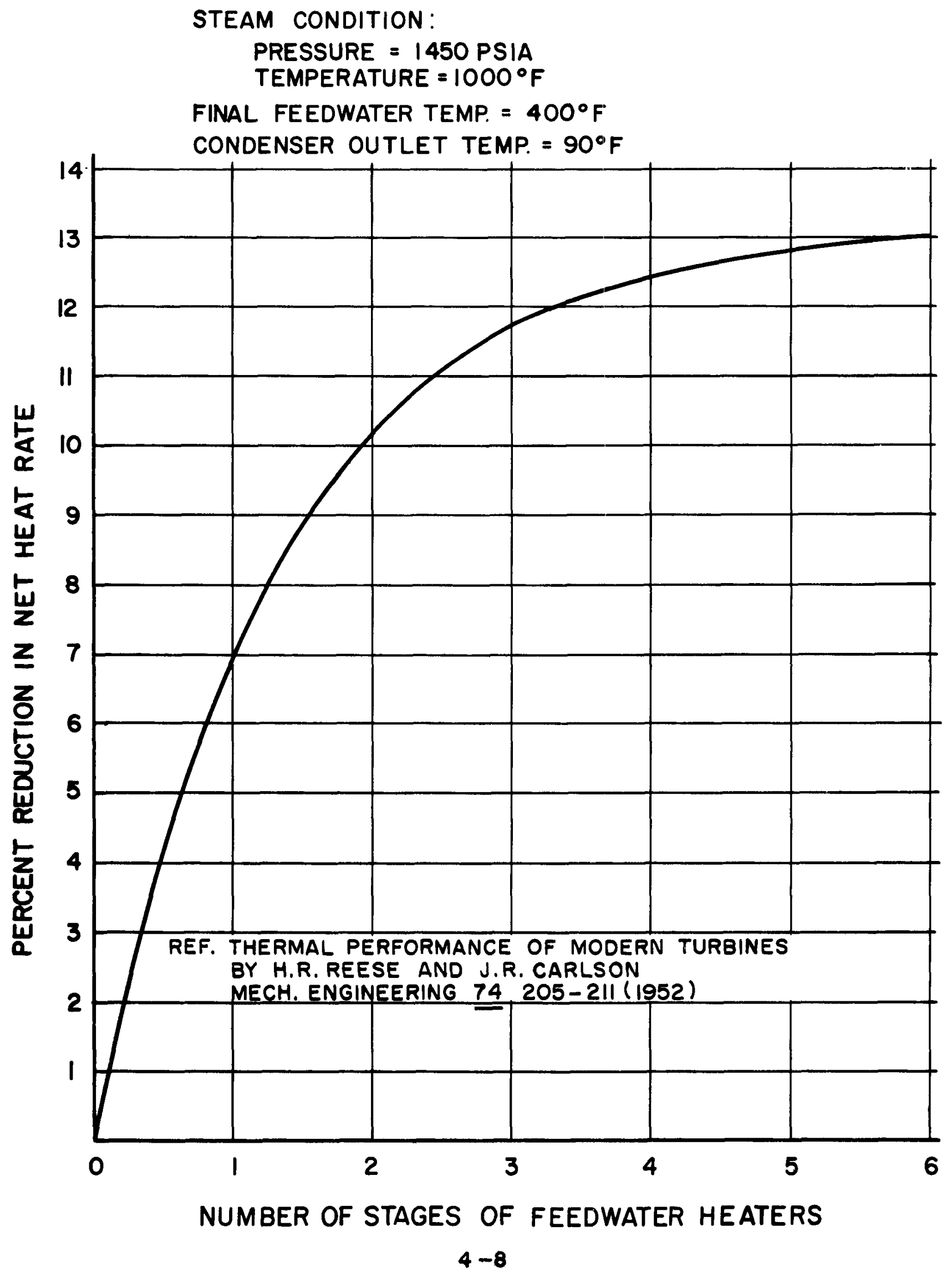


valved too high turbine exit velocities with the consequent high turbine leaving losses making the economics of going to shorter blade lengths unfeasible. The use of longer blade lengths is prohibited by the high moisture content of the steam through the last stages of the turbine.

Table 4.4-1 shows the comparison of a double flow and a triple flow turbine exhaust section with 23 " last stage blade lengths for a $125 \mathrm{MW}$ non-reheat turbine. It is evident that the triple flow exhaust section of the turbine is justifiable on the basis of both the increase in generation due to lower leaving losses, and the better net thermal efficiency. In view of these data, the tandem compound triple flow turbine with 23" last stage blade length was considered to be the most desirable for the purpose of this design.

$4-9$ 
TABLE $4.4-1$

Selection of Turbine Exhaust Section

$\begin{array}{lll}\text { Steam Conditions } & & \\ \quad \text { Flow } & - & 1,055,000 \text { \#/hr } \\ \text { Pressure } & - & 1450 \mathrm{psig} \\ \text { Temp. } & - & 1000^{\circ} \mathrm{F} \\ \text { Final Feedwater Temp. } & - & 400^{\circ} \mathrm{F} \\ \text { Stages of Feedwater } & & \\ \text { Heaters } & - & 4 \\ \text { Turbine Exhaust Pressure } & - & 1.5^{\prime \prime} \mathrm{Hg} \mathrm{Abs} \\ \text { Turbine Type and Speed } & - & \text { Tandem Compound - } \\ & & 3600 \mathrm{rpm} \\ \text { Last Stage Turbine Blade } & & 23 " 1 \\ \text { Length } & - & 80 \% \\ \text { Load Factor } & - & 14 \% \\ \text { Fixed Charges } & - & \$ .15 \mathrm{per} \text { million BTU's } \\ \text { Fuel Cost } & - & \$ 100 / \mathrm{KW} \\ \text { Capability Charge } & -\end{array}$

Type Exhaust Section

Net Station Heat Rate BTU/KWHr

Net Generation at Max. Capability - KW

Annual Fuel Cost

Annual Fuel Cost Difference

Annual Cost due to difference in capability

Additional justified plant investment

Estimated Difference in cost of Plant
Double Flow

9348

123089

$\$ 1,244,102$

0

0

0

0
Triple Flow 9077

126753

$\$ 1,173,152$

$-\$ 70,950$

$-\$ 51,296$

$\$ 873,186$

$\$ 432,000$ 
Section 5.0

Capital Cost Summary 


\subsection{Capital Cost Summary}

This section presents a summary of capital costs, arranged by FPC standard accounts, as applied to a utility which is constructing a power generating unit on its system. The summary is based on the assumption that the necessary research and development has been completed, including operation of a reasonable size prototype through several fuel replacement cycles. Engineering design of all equipment is included in its selling price, in accordance with current practice for conventional power plant equipment. As estimated here, construction is carried out under a cost-plus-a-fixed-fee type contract, thus there are no contingencies and profits of a general contractor.

Clients costs for interest during construction are based on a $31 / 2 \%$ interest rate for a three year construction period.

Clients engineering and administration was estimated at double the usual amount for a conventional station because of special training of personnel, etc.

Escalation was set at $10 \%$ of expenditures for the costs through construction of the plant and job administration.

\section{Summary of Accounts}

$125 \mathrm{MW}-\mathrm{PBR}$

A. Land \& Land Rights

$\$ 350,000$

B. Structures \& Improvements

Turbo-Generator Building

1. Excavation \& Backfill

2. Foundations

3. Structural Steel

4. Miscellaneous Steel

5. Floors

6. Walls

7. Roof

8. Other Building Items

9. Gate House

10. Property Improvements

Total "B" Turbo-Generator Building

$$
\begin{array}{r}
91,000 \\
202,000 \\
150,000 \\
26,000 \\
96,000 \\
94,000 \\
33,000 \\
251,000 \\
3,000 \\
234,000 \\
\hline
\end{array}
$$

$\$ 1,180,000$ 
Containment Vessel Structure

1. Excavation \& Backfill

24,000

2. Foundations

3. Equipment Concrete (Inside Vessel)

82,000

4. Shielding Concrete (Above Grade)

397,000

5. Containment Vessel

126,000

6. Stractural Steel

550,000

17,000

7. Miscellaneous Steel

13,000

8. Other Building Items

130,000

Total "B" Containment Vessel

Structure

$\$ 1,339,000$

Reactor Service Building

1. Excavation \& Backfill

16,000

2. Foundations \& Building Structure

Concrete

158,000

7,000

2,000

6,000

3,000

6. Roof

7. Oth $\in \mathrm{r}$ Building Items

108,000

Total "B" Reactor Service Building

$\$ 300,000$

C. Stearn Generating Plant

Primary System

1. Reactor

2. Shield Water Tank

733,000

57,000

3. Steam Generators

4. Helium Blowers \& Drives

$1,846,000$

$1,194,000$

5. Auxiliary Gas System

6. Decontamination System

418,000

14,000

7. Instruments \& Controls

502,000

8. Main Piping System

489,000

9. Fuel Handling System

10. Aqueous Waste Disposal System

11. Miscellaneous Water Cooling Systems

500,000

300,000

30,000

50,000

50,000

13. Preliminary Operation

50,000

14. Initial Charge of Helium

Total "C" Primary System

$\$ 6,233,000$ 
C. Steam Generating Plant

Secondary System

1. Water Treating System

2. Feed Water Heating \& Pumping

$\$ 208,000$

396,000

3. Instruments \& Controls

330,000

4. Piping

5. Service Water System

$1,135,000$

44,000

6. Preliminary Operation

15,000

Total "C" Secondary System

$\$ 2,128,000$

D. Turbo-Generating Plant

1. Turbine \& Generator Complete

2. Condenser Complete

3. Circulating Water Intake \& Discharge

4. Preliminary Operation

$\$ 4,629,000$

844,000

500,000

30,000

Total "D" Turbo-Generator Plant

$\$ 6,003,000$

E. Accessory Electrical Equipment

1. Generator Main Leads \& Connections

2. Spare Excitation System

3. Station Service Buses \& Switchgear

4. Station Control System

5. Preliminary Operation

6. Cable \& Wire

7. Cable Trays \& Supports

8. Conduit \& Fittings

$\$ 73,000$

160,000

355,000

79,000

17,000

98,000

26,000

46,000

Total "E" Accessory Electrical

Equipment

$\$ 854,000$

F. Transmission Plant

1. Transformers, Switchgear, Accessories

\& Foundations

$\$ 566,000$ 
G. Miscellaneous Power Plant Equipment

1. Compressed Air System

2. Communication System

3. Fire Fighting Equipment - Portable

4. Furniture \& Fixtures

5. Laboratory \& Testing Equipment

6. Lubricating Oil Storage \& Reclaiming

7. Machine Shop Tools \& Equipment

Total "G" Miscellaneous Power

Plant Equipment

$\$ 287,000$

Sub-Total "A" through "G"

$\$ 19,240,000$

H. Omissions \& Contingencies

Turbo-Generator Plant \& Property

Improvements $\quad$ at $5 \%$

Reactor Plant $\quad$ at $30 \%$

Sub-Total "A" through "H"

I. Construction Plant and Job Administration

$$
\text { Sub-Total "A" through "I" }
$$

$\$ \quad 563,000$

$2,392,000$

$\$ 22,195,000$

$\$ 3,329,000$

$\$ 25,524,000$

J. Engineering Design \& Services,

Engineering \& Construction Fees

Sub-Total "A" through "J"

K. Clients Costs:

$\frac{\text { Interest During Construction }}{\text { Engineering and Administration }}$
Escalation

Total
$\$ 2,042,000$

$\$ 27,566,000$

$1,703,000$ 400,000

$2,552,000$

$\$ 32,221,000$ 


\section{$\underline{\text { Summary }}$ \\ $\frac{\text { Turbo-Generator }}{\underline{\text { Plant }}} \quad \frac{\text { Reactor }}{\text { Plant }}$}

A. Land and Land Rights

$\$ 350,000 \$$

$1,180,000$

Turbo-Generator Building

Containment Vessel Building

Reactor Service Building

C. Steam Generating Plant

Primary System

Secondary System

D. Turbo-Generator Plant

E. Accessory Electrical

Equipment

F. Transmission Plant

G. Miscellaneous Power Plant

Equipment
$2,128,000$

$6,003,000$

854,000

566,000

185,000

$\$ 11,266,000$
$1,339,000$

300,000

$6,233,000$

$6,233,000$

$2,128,000$

$6,003,000$

854,000

566,000

102,000

287,000

$1,339,000$

300,000

$1,180,000$

102,000

$\$ 7,974,000$

$\$ 19,240,000$

H. Omissions \& Contingencies

Sub-total A thru $\mathrm{H}$

$\frac{2,955,000}{22,195,000}$

$\frac{3,329,000}{25,524,000}$

Sub-total A thru I

$\frac{2,042,000}{27,566,000}$

Sub-total A thru J

$4,655,000$

K. Clients Costs

$\$ 32,221,000$

Total 